Esquemas de captura de descontinuidades para equações gerais de conservação 



\title{
Esquemas de captura de descontinuidades para equações gerais de conservação
}

\author{
Rodolfo Junior Pérez Narváez
}

Orientador: Prof. Dr. Valdemir Garcia Ferreira

Dissertação apresentada ao Instituto de Ciências Matemáticas e de Computação - ICMC-USP, como parte dos requisitos para obtenção do título de Mestre em Ciências - Ciências de Computação e Matemática Computacional. VERSÃO REVISADA 
Ficha catalográfica elaborada pela Biblioteca Prof. Achille Bassi e Seção Técnica de Informática, ICMC/USP, com os dados fornecidos pelo(a) autor(a)

\begin{tabular}{|c|c|}
\hline \multirow[t]{3}{*}{ P 438e } & $\begin{array}{l}\text { Pérez Narváez, Rodolfo Junior } \\
\text { Esquemas de captura de descontinuidades para } \\
\text { equações gerais de conservação / Rodolfo Junior Pérez } \\
\text { Narváez; orientador Valdemir Garcia Ferreira. -- } \\
\text { São Carlos, } 2013 \text {. } \\
\quad 137 \text { p. }\end{array}$ \\
\hline & $\begin{array}{l}\text { Dissertação (Mestrado - Programa de Pós-Graduação em } \\
\text { Ciências de Computação e Matemática Computacional) -- } \\
\text { Instituto de Ciências Matemáticas e de Computação, } \\
\text { Universidade de São Paulo, } 2013 .\end{array}$ \\
\hline & $\begin{array}{l}\text { 1. esquemas convectivos. 2. leis de conservação. } \\
\text { 3. escoamentos com superfícies livres. 4. Equações de } \\
\text { Navier-Stokes. 5. simulação numérica. I. Garcia } \\
\text { Ferreira, Valdemir, orient. II. Título. }\end{array}$ \\
\hline
\end{tabular}


Este trabalho é dedicado às pessoas que mais amo na vida: minha família. 


\section{Agradecimentos}

A Deus pelo dom da vida e ser meu guia.

A meus pais Rodolfo Pérez C. e Bertha Camargo N. por todos os esforços que fizeram para eu poder conseguir minhas metas. Também a meus irmãos Tommy, Diego y Alejandro.

A meus familiares, em especial a Alfredo R., Jeinny P. e Nakina R. pelo apoio nos momentos difíceis.

A meu orientador e amigo Prof. Dr. Valdemir Garcia Ferreira pela paciência, disposição, orientações e conselhos.

A meus amigos Miguel R., German, Fernando, Henry, Edwin, Miguel C., John, Hugo, Rita, Lucas, Ricardo, Ruben pela ajuda e incentivo nos dias difíceis.

A meus amigos e colegas do LMACC-ICMC pela força e ajuda, em especial Juliana B. pela colaboração e compreensão.

Aos funcionários do ICMC-USP pela disposição e colaboração.

Aos professores do LMACC-ICMC pelos ensinamentos.

Ao Conselho Nacional de Desenvolvimento Científico e Tecnológico-CNPq pelo apoio financeiro.

Finalmente a todos os que direta ou indiretamente contribuíram para a realização deste trabalho. 


\section{Resumo}

Três esquemas de captura de descontinuidade são apresentados para simular sistemas hiperbólicos de leis de conservação e equações de Navier-Stokes incompressíveis, a saber: FDHERPUS (Five Degree Hermite Polynomial Upwind Scheme); RUS (Rational Upwind Scheme); e CSPUS (Cubic Spline Polynomial Upwind Scheme). Esses esquemas são baseados nos critérios de estabilidade CBC e TVD e implementados nos contextos das metodologias diferenças finitas e volumes finitos. A precisão local dos esquemas é verificada acessando o erro e a taxa de convergência em problemas testes de referência. Um estudo comparativo entre os esquemas estudados (incluido o WENO5) e o esquema bem estabelecido de van Albada, para resolver leis de conservação lineares e não lineares, é também realizado. O esquema de convecção que fornece melhores resultados em leis de conservação hiperbólicas é então examinado na simulação de escoamentos de fluidos newtonianos com superfícies livres móveis de complexidade crescente; resultados satisfatórios têm sido observados em termos do comportamento global.

Palavras-chave: esquemas convectivos; captura de choques; termos convectivos; escoamentos com superfícies livres; equações de Navier-Stokes; leis de conservação; simulação numérica. 


\section{Abstract}

Three shock capturing schemes for numerical solution of hyperbolic conservation laws and incompressible Navier-Stokes equations are presented, namely: FDHERPUS (Five Degree Hermite Polynomial Upwind Scheme); RUS (Rational Upwind Scheme); and CSPUS (Cubic Spline Polynomial Upwind Scheme). These schemes are based on CBC and TVD stability criteria and implemented in the context of finite difference and finite volume methodologies. The local observed accuracy of the schemes is verified by assessing the error and convergence rate on benchmark test cases. A comparative study between the schemes (including WENO5) and the well established van Albada scheme to solve standard linear and nonlinear hyperbolic conservation laws is also accomplished. The scheme that has provided better results in hyperbolic conservation laws is then examined in the simulation of Newtonian moving free surface flows of increasing complexity; satisfactory agreement has been observed in terms of the overall behavior.

Key-words: convection schemes; shock capturing; convective terms; free surface flows; NavierStokes equations; conservation laws; numerical simulation. 



\section{Sumário}

1 Introdução 1

2 Formulação matemática $\quad 5$

2.1 Leis de conservação $1 \mathrm{D} \ldots \ldots \ldots \ldots \ldots$

2.1 .1 Equação linear de advecção 1D . . . . . . . . . . . . . . . . . 6

2.1.2 Equação não-linear de Burgers 1D . . . . . . . . . . . . . . . . . . 6

2.1 .3 Equações de Euler 1D . . . . . . . . . . . . . . . . . . . . 7

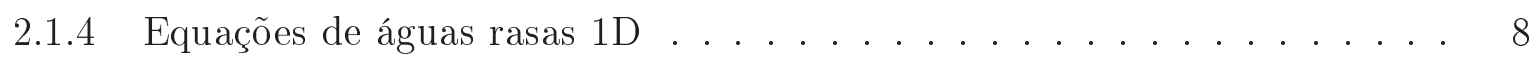

2.2 Leis de conservação 2D . . . . . . . . . . . . . . . . . . . . . . . . . . 8

2.2.1 Equações de Euler . . . . . . . . . . . . . . . . . . . . . . . . 9

2.2.2 Equações de águas rasas . . . . . . . . . . . . . . . . . . . 9

2.3 Equações de Navier-Stokes . . . . . . . . . . . . . . . . . . . . . . . . 10

2.4 Estimativa de Erro . . . . . . . . . . . . . . . . . . . . 12

3 Aproximação para termos convectivos $\quad 15$

3.1 Aproximação dos Termos Convectivos . . . . . . . . . . . . . . . . . 15

3.2 Variáveis normalizadas . . . . . . . . . . . . . . . . . . 16

3.3 Esquemas TVD . . . . . . . . . . . . . . . . . . 18

3.4 Critério de limitação CBC . . . . . . . . . . . . . . . . . . . . . . 19

3.5 Limitadores de fluxo . . . . . . . . . . . . . . . . . . . . . . . 19

3.6 Exemplos de esquemas convectivos . . . . . . . . . . . . . . . . . 23

3.6.1 van Albada (satisfaz as restrições de Spekreijse) . . . . . . . . . . . 23

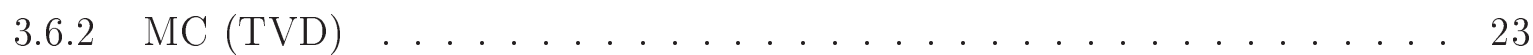

3.6 .3 Minmod $(\mathrm{TVD}) \ldots \ldots \ldots \ldots \ldots . \ldots \ldots$

3.6.4 Superbee (TVD) . . . . . . . . . . . . . . . . . . . 24

3.6 .5 ADBQUICKEST $(\mathrm{TVD}) \ldots \ldots \ldots \ldots \ldots \ldots$

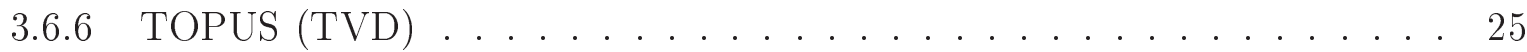


4 Modelagem computacional $\quad 27$

4.1 Discretização das leis de conservação 1D . . . . . . . . . . . . . . . 27

4.1 .1 Equação de advecção 1D . . . . . . . . . . . . . . . . . . 27

4.1 .2 Equação de Burgers 1D . . . . . . . . . . . . . . . . . 28

4.1 .3 Equações de Euler 1D . . . . . . . . . . . . . . . . . . . . . . . . 29

4.1.4 Equações de Euler 2D . . . . . . . . . . . . . . . . . . . 30

4.1.5 Sistema de equações de águas rasas . . . . . . . . . . . . . . . 30

4.2 Equações de Navier Stokes . . . . . . . . . . . . . . . . . . . . . . . 30

4.2.1 Aproximação dos termos convectivos e os esquemas upwind . . . . . . . . 31

4.2.2 Algoritmo computacional . . . . . . . . . . . . . . . 35

5 Derivação dos esquemas convectivos $\quad 37$

5.1 O esquema FDHERPUS . . . . . . . . . . . . . . . . 37

5.2 O esquema CSPUS . . . . . . . . . . . . . . . . . 41

5.3 O Esquema RUS . . . . . . . . . . . . . . . . . . . 46

6 Resultados numéricos 1D $\quad 51$

6.1 Equação linear de advecção . . . . . . . . . . . . . . . . . . . 51

6.2 Equação não-linear de Burgers . . . . . . . . . . . . . . . . . . 58

6.3 Equações de Euler . . . . . . . . . . . . . . . . . . . . . . 64

6.4 Problemas modelados pelas equações de águas rasas . . . . . . . . . . . . . 72

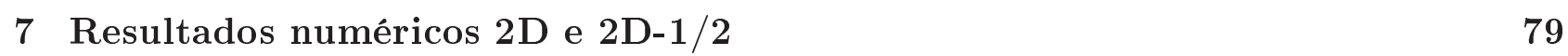

7.1 Equações de Euler . . . . . . . . . . . . . . . . . . . . 79

7.2 Sistema não-linear de águas rasas . . . . . . . . . . . . . . . . . . . 93

7.3 Escoamentos incompressíveis laminares 2D e 2D-1/2 . . . . . . . . . . . . 101

7.3.1 Jato livre 2D sobre uma superfície rígida impermeável . . . . . . . . . . . 101

7.3.2 Colapso de uma coluna 2D de fluido . . . . . . . . . . . . . . . 103

7.3.3 Experimento de Taylor no caso 2D-1/2 . . . . . . . . . . . 108

7.3.4 Ressalto hidráulico circular no caso 2D-1/2 . . . . . . . . . . 113

8 Resultados numéricos de problemas 3D complexos $\quad 117$

8.1 Colapso de um bloco de fluido . . . . . . . . . . . . . . . . 117

8.2 Ressalto hidráulico circular . . . . . . . . . . . . . . . . 120

8.3 Jato planar oscilante . . . . . . . . . . . . . . . . 121

9 Considerasões finais e trabalhos futuros $\quad 125$ 


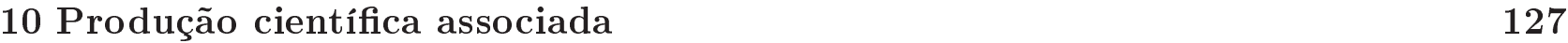

10.1 Artigos e resumos . . . . . . . . . . . . . . . . . . . 127

10.1.1 Artigos publicados anais de congressos . . . . . . . . . . . . 127

10.1.2 Artigos submetidos em periódicos . . . . . . . . . . . . 130

$\begin{array}{lll}\text { A CLAWPACK } & 131\end{array}$

Referências Bibliográficas 132 


\section{Lista de Figuras}

3.1 Localização dos nós computacionais $D, U$ e $R$ em função da velocidade convectiva

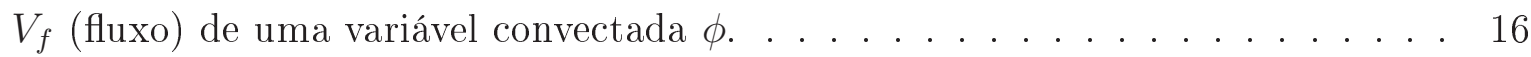

3.2 Diagrama de variáveis normalizadas mostrando os esquemas QUICK, FOU e Diferença Central. . . . . . . . . . . . . . . . . 17

3.3 Diagrama CBC em variáveis normalizadas . . . . . . . . . . . . . . . 20

3.4 Diagrama de limitador de fluxo - Diagrama de Sweby. . . . . . . . . . . . . . 21

3.5 Região TVD em Variáveis Normalizadas. . . . . . . . . . . . . . . . . . . . 22

3.6 Região de monoticidade de Spekreijse. . . . . . . . . . . . . . . . . . . . . 23

4.1 Representação esquemática em que se mostra as aproximações dos termos convectivos, mostrando as faces envolvidas $f$ e $g$ para a aproximação e a direção da velocidade $V_{f}\left(V_{g}\right)$ de convecção na face $f(g) \ldots \ldots \ldots \ldots$

4.2 Célula computacional típica mostrando onde as variáveis dependentes são avaliadas. 31

5.1 Esquema FDHERPUS na região TVD em variáveis normalizadas. . . . . . . . . 40

5.2 Limitador de fluxo para o esquema FDHERPUS na região TVD. . . . . . . . . . 41

5.3 O esquema CSPUS em variáveis normalizadas dentro da região TVD. . . . . . . 45

5.4 Limitador de fluxo correspondente ao esquema CSPUS na região TVD. . . . . . 47

5.5 O limitador de fluxo RUS e a região de monoticidade de Spekreijse. . . . . . . . 48

5.6 O esquema RUS dentro da região TVD . . . . . . . . . . . . . . . . . . 49

6.1 Soluções exata e numéricas para a equação linear de advecção: (a) aproximações obtidas pelos esquemas: van Albada, RUS, FDHERPUS, CSPUS, MRUS para $\epsilon=10^{-7}$ e $\epsilon=10^{-8}$, (b) e (c) detalhes do comportamento dos esquemas em regiões específicas. . . . . . . . . . . . . . . . . . . . 5 52

6.2 Erro na norma $L_{1}$ e na escala log-log para os esquemas van Albada, RUS, FDHERPUS, CSPUS e MRUS com $\epsilon=10^{-7}$ e $\epsilon=10^{-8} \ldots \ldots \ldots \ldots$. . . . . 54 
6.3 (a) Soluções exata e numéricas para a equação linear de advecção com condição inicial (6.2). As aproximações são obtidas pelos esquemas: van Albada, RUS, FDHERPUS, CSPUS, MRUS para $\epsilon=10^{-7}$ e $\epsilon=10^{-8}$. Detalhes das regiões demarcadas são mostrados em (b), (c), (d) e (e) . . . . . . . . . . . 56

6.4 Variação total para o Teste 2 com respeito a tempo t. . . . . . . . . . 58

6.5 Evolução da condição inicial (6.3) ao longo do tempo para o limitador de fluxo correspondente ao esquema FDHERPUS. . . . . . . . . . . . . . . . 59

6.6 (a) Soluções exata e numéricas para equação não-linear de Burgers antes e após do choque. As aproximações são obtidas pelos esquemas: van Albada, RUS, FDHERPUS, CSPUS, MRUS para $\epsilon=10^{-7}$ e $\epsilon=10^{-8}$. Detalhes são mostrados em $(b),(c),(d)$ e $($ e) . . . . . . . . . . . . . . . . . .

6.7 Detalhes da solução exata e dos resultados computacionais para equação não-linear de Burgers no caso viscoso mostrando o aumento de algumas regiões. As aproximações são obtidas pelos esquemas: van Albada, RUS, FDHERPUS, CSPUS, MRUS para $\epsilon=10^{-7}$ e $\epsilon=10^{-8}$.

6.8 Análise da TV feita para o Teste 4, mostrando o comportamento dos esquemas.

6.9 (a) Solução da equação de Euler 1D para o teste de Shu-Osher com $N=200$ e $C=0.5$. (b) Região de ampliação. . . . . . . . . . . . . . . . . . . .

6.10 (a) Solução da equação de Euler 1D para o teste de Shu-Osher com $N=200$ e $C=0.9$. (b) Região de ampliação. . . . . . . . . . . . . . . .

6.11 (a) Solução da equação de Euler 1D para o teste de Shu-Osher com $N=400$ e $C=0.1$. (b) Região de ampliação. . . . . . . . . . . . . . . . .

6.12 (a) Solução da equação de Euler 1D para o teste de Shu-Osher com $N=400$ e $C=0.8$. (b) Região de ampliação. . . . . . . . . . . . . . . . .

6.13 (a) Solução da equação de Euler 1D para o teste de Shu-Osher com $N=800$ e $C=0.1$. (b) Região de ampliação. . . . . . . . . . . . . . . . .

6.14 (a) Solução da equação de Euler 1D para o teste de Shu-Osher com $N=800$ e $C=0.5$. (b) Região de ampliação. . . . . . . . . . . . . . . . 66

6.15 Soluções de referência e numéricas (globais e detalhes) para a densidade $\rho$ no problema envolvendo fortes interações de choques. . . . . . . . . . . . 68

6.16 Soluções de referência e numéricas (globais e detalhes) para a energia $E$ no problema envolvendo fortes interações de choques. . . . . . . . . . . . . . . 69

6.17 Soluções de referência e numéricas (globais e detalhes) para a velocidade $u$ no problema envolvendo fortes interações de choques. . . . . . . . . . . . . 70

6.18 Evolução da profundidade $h$ (coluna à esquerda) e da vazão hu (coluna à direita). 73

6.19 Problema Dam-break 1D . . . . . . . . . . . . . . . . . 74

6.20 Evolução temporal para o problema de Dam-break. Ilustra-se as soluções exata e numérica na variável $h \ldots \ldots \ldots \ldots \ldots \ldots \ldots$ 
6.21 Evolução temporal para o problema de Dam-break. Ilustra-se as soluções exata e numérica na variável $h u . \ldots \ldots \ldots$. . . . . . . . . . . 76

6.22 Análise TVD para o Teste 7 para os esquemas van Albada, RUS, FDHERPUS e CSPUS.

7.1 Aproximações obtidas com os esquemas van Albada, RUS, FDHERPUS, CSPUS e MRUS, com $\epsilon=10^{-7} \ldots \ldots \ldots \ldots \ldots$. . . . . . . . . . . 80

7.2 Jato astrofísico propagando-se num ambiente a velocidade adimensional $u=30 . \quad 82$

7.3 Resultados numéricos obtidos com o uso do esquema minmod. . . . . . . . . . . 83

7.4 Resultados numéricos obtidos com o uso do esquema van Albada. . . . . . . . . 83

7.5 Resultados numéricos obtidos com o uso do esquema FDHERPUS. . . . . . . . . 84

7.6 Resultados numéricos obtidos com o uso do esquema RUS. . . . . . . . . . . . 84

7.7 Resultados numéricos obtidos com o uso do esquema CSPUS. . . . . . . . . . 85

7.8 Resultados numéricos obtidos com o uso do esquema MRUS. . . . . . . . . . . . 85

7.9 Densidade $\rho$ ao longo da reta $y=0.5$ para o problema do jato astrofísico. . . . 86

7.10 Contorno da densidade para o problema de interação entre quatro choques usando $200 \times 200$ células computacionais. . . . . . . . . . . . . . . . . 88

7.11 Contorno da densidade $\rho$ ao longo da reta $y=x$ para problema de interação entre quatro choques mostrando regiões de ampliação. . . . . . . . . . . . . . 89

7.12 Regiões de ampliação da Figura 7.11. . . . . . . . . . . . . . . . . . . 89

7.13 Contorno da densidade $\rho$ para o problema de interação entre quatro choques usando a malha de $1000 \times 1000$ células computacionais. . . . . . . . . . . . . . . 91

7.14 Domínio e condições iniciais para problema de RT. . . . . . . . . . . . . . . . 92

7.15 Contornos da densidade para o problema das instabilidades de Raylegh-Taylor. Linhas de contorno vão de $\rho=0.952269$ até $\rho=2.14589$. . . . . . . . . . . . 94

7.16 Contornos da densidade para o problema das instabilidades de Raylegh-Taylor. Linhas de contorno vão de $\rho=0.952269$ até $\rho=2.14589$.

7.17 Profundidade da água $h$ para o problema de ruptura de uma barragem radial nos tempos $t=0$ e $t=0.25 \ldots \ldots \ldots \ldots$. . . . . . . . . . . . . . . .

7.18 Evolução do problema de ruptura de uma barragem radial para o esquema FDHERPUS. . . . . . . . . . . . . . . . . . . . . . 98

7.19 Altura $h$ para o problema de ruptura de uma barragem radial no plano $x \perp y$. . 100

7.20 Comparação dos resultados obtidos sobre a reta $y=0$ para o problema ruptura de uma barragem radial. . . . . . . . . . . . . . . . . . . . . 100

7.21 Ampliação das regiões destacadas na Figura 7.20 para o problema ruptura de uma barragem radial. . . . . . . . . . . . . . . . . . . 101

7.22 Ilustração esquemática para o problema de um jato livre sobre uma superfície rígida impermeável. . . . . . . . . . . . . . . . . . . . . . . . . . . 102 
7.23 Contorno da velocidade na direção $x$ para o problema de jato livre sobre uma superfície livre impermeável. . . . . . . . . . . . . . . . . . . . . . . . . . . 103

7.24 Contorno da velocidade na direção y para o problema de jato livre sobre uma superfície livre impermeável. . . . . . . . . . . . . . . . . . . . . . . . . 104

7.25 Contorno da pressão para o problema de jato livre sobre uma superfície livre impermeável.

7.26 Comparação entre as soluções numéricas com o esquema FDHERPUS e a solução analítica de Watson (1964) [82] para a altura H . . . . . . . . . . . . . . . 105

7.27 Representação esquemática para o problema do colapso de uma coluna de fluido. 106

7.28 Contorno da pressão para o problema do colapso de uma coluna de fluido. . . . 106

7.29 Contorno da velocidade em $x$ para o problema do colapso de uma coluna de fluido.107

7.30 Contorno da velocidade em y para o problema do colapso de uma coluna de fluido.107

7.31 Comparação das soluções teórica, experimental e numéricas para o problema de colapso de uma coluna de fluido. . . . . . . . . . . . . . . . . . . . 108

7.32 Representação esquemática para o problema de Taylor. . . . . . . . . . . . . . 109

7.33 Resultados experimentais e numéricos para o experimento de Taylor nos tempos $t=0.75 \mathrm{~s}$ e $t=2.5 \mathrm{~s}$. O esquema FDHERPUS foi adotado nas simulações. . . . . 110

7.34 Resultado numérico para o experimento de Taylor obtido com o uso do esquema FDHERPUS no tempo $t=10.0 \mathrm{~s} . \ldots \ldots \ldots \ldots \ldots 11$

7.35 Contornos de pressão (caso (a)), velocidade na direção $x$ (caso (b)) e velocidade na direção $y$ (caso (c)) para o experimento de Taylor obtidos com esquema

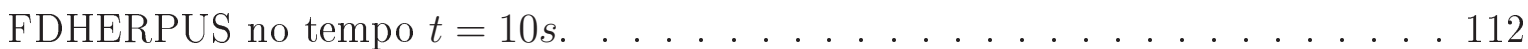

7.36 Representação esquemática para o problema de ressalto hidráulico circular. . . 113

7.37 Comparação entre as soluções analítica de Watson (1964) [82] e numéricas obtidas pelo esquema FDHERPUS para o problema de ressalto hidráulico circular. . 115

7.38 Ilustração dos resultados experimentais (fig. (a)) e numérico (fig. (b)) para o problema de ressalto hidráulico circular.

8.1 Representação esquemática do colapso de um bloco de fluido sob à ação da gravidade. . . . . . . . . . . . . . . . . . . . . 118

8.2 Campo da pressao para o problema de colapso de um bloco de fluido. . . . . . . 118

8.3 Campo da velocidade na direção $x$ para o problema de colapso de um bloco de fluido.

8.4 Campo da velocidade na direção $z$ para o problema de colapso de um bloco de fluido.

8.5 Comparação das soluções numéricas obtida com o FDHERPUS e os resultados consagrados da literatura.

8.6 Comparação qualitativa entre os resultados experimental e numérico para o problema de ressalto hidráulico circular. 
8.7 Evolução do escoamento mostrando a formação da descontinuidade. . . . . . . . 121

8.8 Representação esquemática para a simulação de um jato planar oscilante. . . . . 122

8.9 Resultados para o esquema FDHERPUS para o fenômeno do jato planar oscilante.123

8.10 Simulação computacional de [80] para um jato planar oscilante. . . . . . . . . . 124

8.11 Resultado experimental de [54] para um jato planar oscilante. . . . . . . . . . 124 


\section{Lista de Tabelas}

6.1 Erro relativo $E_{h}$ e estimativa para a ordem de convergência $q$. . . . . . 53

6.2 Erro relativo $E_{h}$ e estimativa para a ordem de convergência $q$. . . . . 57

6.3 Erro relativo $E_{h}$ e estimativa para a ordem de convergência $q$. . . . . . 61

6.4 Erro relativo $E_{h}$ e estimativa para a ordem de convergência $q$. . . . . . 71

6.5 Erro relativo $E_{h}$ e estimativa para a ordem de convergência $q$. . . . . 77

7.1 Erro relativo $E_{h}$ e estimativa para a ordem de convergência $q$. . . . . 81 
CAPÍTULO

\section{1}

\section{Introdução}

Muitas equações da dinâmica dos fluidos de interesse em engenharia, desde as equações de conservação para o transporte de escalares até as equações de Navier-Stokes tridimensionais a altos valores do número de Reynolds, são dominadas por convecção. Como resultado tais sistemas são propensos à formação de frentes abruptas, com soluções com descontinuidades (ou choques) sendo a principal causa de dificuldades numéricas. Um cuidado especial deve, portanto, ser tomado quando a discretização dos termos convectivos é levada a cabo. Em particular, um esquema upwind de captura de descontinuidades deve tornar a formulação discreta global tão precisa quanto possível, deve ser estável (se possível incondicionalmente) e ser de fácil implementação. Além disso, o esquema convectivo deve ser robusto e útil para reproduzir soluções com significado físico em períodos curtos de tempo durante os quais o regime transiente frequentemente ocorre.

O conflito entre se obter precisão e limitação das soluções numéricas de problemas complexos em CFD (Computational Fluid Dynamics) ainda permanece. De um lado, os esquemas upwind clássicos de baixa ordem, tais como os esquemas FOU (First Order Upwind) (1952) [14] e HDS (Hybrid Differencing Scheme) (1972) [65], são estratégias incondicionalmente estáveis; mas tendem a atenuar os processos de transporte por meio do acúmulo de difusão numérica. Por outro lado, aproximações clássicas de alta ordem, tais como os esquemas DC (Diferenças Centrais) e QUICK (Quadratic Upstream Interpolation for Convective Kinematics) (1979) [36], são boas estratégias para melhorar a precisão do método numérico. Entretanto, esses esquemas introduzem oscilações espúrias (wiggles) nas representações discretas comprometendo os resultados.

Nas últimas décadas uma variedade de esquema upwind para aproximar termos advectivos gerais tem sido propostos, dentre os quais pode-se citar o esquema de van Albada (1982) [75] e os esquemas WACEB (Weighted-Average Coefficient Ensuring Boundedness) de Song et 
al. (2000) [45], CUBISTA (Convergent and Universally Bounded Interpolation Scheme for the Treatment of Advection) de Alves et al. (2003) [1] e ADBQUICKEST (ADaptative Bounded Quadratic Upstream Interpolation for convective Kinematics with Estimated Streaming Terms) de Ferreira et al. (2009) [20]. Esses esquemas convencionais têm fornecido soluções aceitáveis para problemas multidimensionais não lineares e têm mostrado ser computacionalmente mais econômicos que os esquemas consagrados ENO (Essentially Non-Oscillatory) (1989) [63] (e os relacionados WENO (Weighted ENO) (1996, 2000) [34, 3], desde que eles proporcionem informações úteis em malhas grosseiras. A inconveniência desses esquemas é que eles não são bem adaptados para resolver problemas de grande complexidade tais como escoamentos de fluidos com superfícies livres móveis em uma vasta gama do número de Reynolds. Há também dois esquemas convectivos relevantes, a saber o esquema TOPUS (Third Order Polynomial Upwind Scheme) de Ferreira et al. (2012) [22] e o esquema SDPUS-C1 (Six Degree Polynomial Upwind Scheme of C1 Class) de Lima et al. (2012) [43], os quais apareceram recentemente na literatura especializada. Ambos os esquemas TOPUS and SDPUS-C1 podem apresentar problemas de diferenciabilidade quando altos valores do número de CFL (Courant Friedrichs Lewy) são impostos; TOPUS não é continuamente diferenciável, desde que nas vizinhanças dos pontos $(0,0)$ e $(1,1)$ no diagrama de variáveis normalizadas de Leonard (1988) [37] ele não é suave, enquanto o esquema SDPUS-C1 é apenas de classe $\mathrm{C}^{1}$. Na prática, alta classe de diferenciabilidade para um esquema upwind é, de acordo com Lin and Chieng (1991) [44], essencial para se conseguir convergência a grandes passos no tempo.

Assim, a necessidade de novos esquemas upwind para captura de descontinuidades que superem as inconveniências mencionadas anteriormente e que proporcionam boa precisão (maior ou igual a dois), robustez, limitação e simplicidade de implementação é um tema atual e contemplado nesta dissertação de mestrado. Essa necessidade tem sido a principal motivação para o presente estudo. Outra motivação para o estudo é o desejo em se obter soluções numéricas convergentes para um largo espectro de problemas complexos usando novos esquemas upwind com uma molécula computacional fixa de três pontos por fluxo numérico. Em particular, o projeto está relacionado à solução numérica de leis de conservação hiperbólicas e de escoamentos incompressíveis em regime transitório com superfícies livres móveis. Atenção especial é voltada à derivação de algoritmos upwind de convecção para essas duas classes de problemas, e são propostos neste trabalho os seguintes esquemas convectivos: o FDHERPUS (Five Degree Hermite Polynomial Upwind Scheme), o RUS (Rational Upwind Scheme) e o CSPUS (Cubic Spline Polynomial Upwind Scheme) (há ainda uma modificação do esquema RUS nomeada como MRUS e derivada em função do aparecimento de extremos).

As soluções numéricas para as leis de conservação tratadas neste estudo foram geradas com o uso do código CLAWPACK (Conservation LAWs PACKage) de LeVeque (2006) [42]. O código resolve numericamente uma variedade de sistemas hiperbólicos e está implementado em linguagem FORTRAN, no contexto do método de volumes finitos, permitindo resolver 
problemas de Riemann com condições iniciais (suaves e não suaves) e de contorno bem definidas. No código estão implementados o método de Godunov de primeira ordem com termo de correção e o esquema de LeVeque (2004) [41]. Fundamentalmente, o CLAWPACK foi equipado com os correspondentes limitadores de fluxo para os esquemas FDHERPUS, RUS e CSPUS. No caso da simulação de problemas de escoamentos incompressíveis com superfície livres móveis, as equações de conservação foram discretizadas utilizando-se o método de diferenças finitas sobre malhas diferenciadas. A metodologia numérica utilizada no projeto é uma variante do método de projeção de Chorin (1968) [11], proposto originalmente por Harlow e Welch (1965) [29] (método MAC) e bem discutido por Peyret e Taylor (1983) [48]. Baseado no método MAC, o presente projeto utilizará formulações explícitas (com o método de Euler para a marcha no tempo) para a solução numérica de problemas modelados pelas equações instantâneas de Navier-Stokes bi- e tridimensionais. O ambiente de simulação genuinamente brasileiro Freeflow de Castelo et al. (2000) [10], desenvolvido no LMACC (Laboratório de Matemática Aplicada e Computação Científica) do ICMC-USP, foi o código base para a simulação computacional de problemas complexos envolvendo fronteiras livres móveis.

A dissertação está dividida em 10 capítulos e um apêndice descritos como segue:

- No Capítulo 2, são apresentadas as equações diferenciais parciais utilizadas para testar os esquemas numéricos;

- No Capítulo 3, descreve-se a base teórica para o desenvolvimento de esquemas de alta resolução e seus respectivos limitadores de fluxo;

- No Capítulo 4, mostram-se as discretizações das equações instantâneas de Navier-Stokes na formulação incompressível, incluindo o tratamento dos termos convectivos e uma descrição da metodologia numérica utilizada;

- No capítulo 5, é apresentado o desenvolvimento dos esquemas numéricos upwind;

- No Capítulo 6, são apresentados os resultados numéricos para leis de conservação hiperbólicas 1D;

- No Capítulo 7, são apresentados os resultados numéricos para leis de conservação hiperbólicas 2D e para escoamentos incompressíveis com superfícies livres móveis 2D e 2D-1/2;

- No Capítulo 8, são mostrados os resultados numéricos 3D para escoamentos incompressíveis com superfície livre móveis no regime laminar;

- No Capítulo 9, apresentam-se as considerações finais e trabalhos futuros;

- No Capítulo 10, apresenta-se uma lista das publicações em congressos; 
- No Apêndice, apresenta-se de maneira sintética o algoritmo implementado no software CLAWPACK para a resolução de leis de conservação hiperbólicas. 
CAPÍTUlO

\section{Formulação matemática}

Neste capítulo são apresentadas/discutidas as equações diferenciais parciais (EDPs) básicas as que são resolvidas numericamente neste trabalho de mestrado. Em particular, algumas leis de conservação hiperbólicas e as equações de Navier-Stokes na formulação incompressível são descritas.

\subsection{Leis de conservação 1D}

Durante as últimas décadas houve uma enorme quantidade de atividade relacionada à construção de soluções aproximadas de EDPs, em grande parte para leis de conservação não-lineares que modelam vários fenômenos físicos, tais como aqueles em dinâmica dos gases, em acústica, geofísica, biomecânica, aerodinâmica, astrofísica, engenharia de tráfego, entre muitos outros (veja por exemplo Thole (1986) [70], Leveque (2004) [41] e De Azevedo (2004) [17]). Estas leis constituem um sistema de EDPs, com estructura particularmente simples para o caso 1D e em forma conservativa é dado como

$$
\frac{\partial \boldsymbol{U}}{\partial t}+\frac{\partial \boldsymbol{F}(\boldsymbol{U})}{\partial x}=0
$$

em que $\boldsymbol{U}=\left(u_{1}, u_{2}, \ldots, u_{N}\right)^{T} \in \mathbb{R}^{N}$ é o vetor de quantidades conservadas, enquanto que os componentes do vetor fluxo $\boldsymbol{F}(\boldsymbol{U})=\left(f_{1}, f_{2}, \ldots, f_{N}\right)^{T}$, correspondem aos fluxos (ver detalhes em Bressan (2011) [7]). A equação (2.1) é equivalente ao sistema quase-linear

$$
\frac{\partial \boldsymbol{U}}{\partial t}+\boldsymbol{A}(\boldsymbol{U}) \frac{\partial \boldsymbol{U}}{\partial x}=0
$$

onde $\boldsymbol{A}(\boldsymbol{U})=\frac{\partial \boldsymbol{F}}{\partial \boldsymbol{U}}$ é a matriz jacobiana do vetor fluxo $\boldsymbol{F}$ com autovalores reais $\lambda_{1}<\lambda_{2}<\cdots<$ 
$\lambda_{n}$. A seguir são apresentadas a equação linear de advecção, a equação não-linear de Burgers e os sistemas hiperbólicos não-lineares de Euler da dinâmica dos gases e águas rasas.

\subsubsection{Equação linear de advecção 1D}

A equação linear de advecção modela ondas que "arrastram" consigo propriedades físicas do meio por onde se propagam. Por exemplo, em engenharia mecânica ou engenharia química, advecção é um mecanismo de transporte de uma substância ou uma propriedade conservada de um fluido em movimento (ver por exemplo LeVeque (2004) [41]).

Esta equação tem a forma

$$
\frac{\partial \boldsymbol{U}}{\partial t}+\frac{\partial \boldsymbol{F}(\boldsymbol{U})}{\partial x}=0, \quad x \in\left[x_{L}, x_{R}\right] \quad \text { e } \quad t \geq 0
$$

Considera-se o vetor de quantidades conservadas $\boldsymbol{U}=u$ e a função fluxo é dada como $\boldsymbol{F}(\boldsymbol{U})=$ au, com a constante, denominada constante de adveç̧ão. As condições iniciais e de contorno associadas a este problema são respectivamente dadas por

$$
\begin{gathered}
u(x, 0)=u_{0}(x), \\
u\left(x_{L}, t\right)=u_{L}, \quad u\left(x_{R}, t\right)=u_{R} .
\end{gathered}
$$

Como o tempo evolui, os dados iniciais propagam-se para a direita (se $a>0$ ) ou para esquerda (se $a<0)$ com velocidade $a$. A solução exata de $(2.3)$ é $u(x, t)=u_{0}(x-a t)$, sendo as retas $x-a t=x_{0}$ conhecidas como retas características da equação (ver, por exemplo, LeVeque (1992) [39]).

\subsubsection{Equação não-linear de Burgers 1D}

Um das mais importantes EDPs na teoria de leis de conservação não-lineares é a equação de Burgers, dada por

$$
\frac{\partial \boldsymbol{U}}{\partial t}+\frac{\partial \boldsymbol{F}(\boldsymbol{U})}{\partial x}=\boldsymbol{S}, \quad x \in\left[x_{L}, x_{R}\right] \quad \text { e } \quad t \geq 0
$$

em que o vetor de quantidades conservadas $\boldsymbol{U}=u$ e o vetor fluxo é dado por $\boldsymbol{F}(\boldsymbol{U})=\frac{u^{2}}{2}$. Considera-se nesse trabalho os casos em que $\boldsymbol{S}=\nu \frac{\partial^{2} u}{\partial x^{2}} \operatorname{com} \nu>0$ (caso viscoso) e $\nu$ é a constante que define a viscosidade cinemática. Se essa viscosidade for nula, a equação (2.6) é conhecida como equação não viscosa de Burgers. As condições iniciais e de contorno do 
problema são respectivamente:

$$
\begin{gathered}
u(x, 0)=u_{0}(x), \\
u\left(x_{L}, t\right)=f\left(x_{L}, t\right), \quad u\left(x_{R}, t\right)=g\left(x_{R}, t\right) .
\end{gathered}
$$

O estudo desta equação tem sido um ponto chave para o desenvolvimento da teoria de ondas de choque e ondas de difusão para sistemas viscosos e não viscosos de leis de conservação. A equação de Burgers para o caso viscoso pode ser vista como um protótipo unidimensional da equação de Navier-Stokes e neste caso a incógnita $u=u(x, t)$ é interpretada como sendo a velocidade unidimensional de uma partícula de fluido na posição $x$ e no instante $t$.

\subsubsection{Equações de Euler 1D}

Em dinâmica dos fluidos, as equações de Euler descrevem o movimento de um fluido compressível e não viscoso. Estas são dadas como

$$
\frac{\partial \boldsymbol{U}}{\partial t}+\frac{\partial \boldsymbol{F}(\boldsymbol{U})}{\partial x}=0, \quad x \in\left[x_{L}, x_{R}\right] \quad \text { e } \quad t \geq 0,
$$

em que o vetor de quantidades conservadas $\boldsymbol{U}$ e o vetor fluxo $\boldsymbol{F}(\boldsymbol{U})$ são dadas, respectivamente, como

$$
\begin{gathered}
\boldsymbol{U}=(\rho, \rho u, E)^{T}, \\
\boldsymbol{F}(\boldsymbol{U})=\left(\rho u, \rho u^{2}+p, u(E+p)\right)^{T} .
\end{gathered}
$$

Este sistema de três leis de conservação represemtam as equações de Euler para a dinâmica dos gases, onde $\rho$ é a densidade, $\rho u$ é a quantidade de momento, $E$ é a energia total e $p$ é a pressão. Além disso, para fechar o sistema é considerada a equação do gás ideal $p=(\gamma-1)\left(E-\frac{1}{2} \rho u^{2}\right)$, na qual $\gamma=1.4$ é a razão do calor específico (ver Harten (1989) [31]). As condições inicias para do sitema são dadas como

$$
\left(\rho_{0}, u_{0}, p_{0}\right)^{T}= \begin{cases}\left(\rho_{L}, u_{L}, p_{L}\right)^{T}, & x \leq x_{0} \\ \left(\rho_{R}, u_{R}, p_{R}\right)^{T}, & x>x_{0}\end{cases}
$$

A condição de contorno adotada é a extrapolação de ordem zero (para detalhes ver LeVeque (2004) [41]). Este sistema de EDPs tem sido amplamente utilizado na literatura para investigar o desempenho de esquemas de alta resolução upwind, ver por exemplo Ferreira (2009) [21]. 


\subsubsection{Equações de águas rasas 1D}

Fluxos envolvendo superfície livre, muito comuns em hidráulica, são usualmente descritos por meio das equações de águas rasas, levando em conta que as dimensões verticais representativas sejam pequenas em relação as dimensões horizontais. Apesar de sua simplicidade, esta descrição é válida em muitas aplicações práticas. Assim, as equações unidimensionais que modelam a dinâmica de um fluido num canal aberto são:

$$
\frac{\partial \boldsymbol{U}}{\partial t}+\frac{\partial \boldsymbol{F}(\boldsymbol{U})}{\partial x}=\boldsymbol{S}, \quad x \in\left[x_{L}, x_{R}\right] \quad \text { e } \quad t \geq 0
$$

em que o vetor de quantidades conservadas $\boldsymbol{U}$ e o vetor fluxo $\boldsymbol{F}(\boldsymbol{U})$ são dadas respectivamente como

$$
\begin{gathered}
\boldsymbol{U}=(h, h u)^{T}, \\
\boldsymbol{F}(\boldsymbol{U})=\left(h u, h u^{2}+\frac{1}{2} g h^{2}\right)^{T} .
\end{gathered}
$$

onde $x$ é a coordenada ao longo do canal, $u$ é a velocidade do fluido, $h$ é a profundidade do fluido no canal, $h u$ é a vazão e $g$ a gravidade. As condições iniciais são dadas por

$$
u(x, 0)=u_{0}(x) \quad \text { e } \quad h(x, 0)=h_{0}(x),
$$

e as condições de contorno comumente utilizadas, segundo LeVeque (2004) [41], são extrapolação de ordem zero.

\subsection{Leis de conservação $2 \mathrm{D}$}

As leis de conservação hipebólicas 2D consideradas neste trabalho são da forma

$$
\frac{\partial \boldsymbol{U}}{\partial t}+\frac{\partial \boldsymbol{F}(\boldsymbol{U})}{\partial x}+\frac{\partial \boldsymbol{G}(\boldsymbol{U})}{\partial y}=0
$$

onde $\boldsymbol{U}: \mathbb{R}^{2} \times \mathbb{R} \longrightarrow \mathbb{R}^{N}$ é o vetor de quantidades conservadas e $\boldsymbol{F}, \boldsymbol{G}$ são vetores funções fluxo. Apresenta-se nessa seção as equações não-lineares de Euler e o sistema não-linear de águas rasas. 


\subsubsection{Equações de Euler}

As leis de conservação hiperbólicas de Euler com termo fonte $\boldsymbol{S}$ tem sido objeto de intensa investigação nas últimas décadas. Isso deve-se a suas muitas aplicações como por exemplo formação estelar, previsão do tempo, entre outras. No caso 2D, as equações de Euler são escritas da forma

$$
\frac{\partial \boldsymbol{U}}{\partial t}+\frac{\partial \boldsymbol{F}(\boldsymbol{U})}{\partial x}+\frac{\partial \boldsymbol{G}(\boldsymbol{U})}{\partial y}=\boldsymbol{S}, \quad(x, y) \in\left[x_{L}, x_{R}\right] \times\left[y_{B}, y_{T}\right], \quad t \geq 0,
$$

onde o vetor de quantidades conservadas $\boldsymbol{U}$ e vetores fluxos $\boldsymbol{F}(\boldsymbol{U})$ e $\boldsymbol{G}(\boldsymbol{U})$ são dadas, respectivamente, como

$$
\begin{gathered}
\boldsymbol{U}=(\rho, \rho u, \rho v, E)^{T}, \\
\boldsymbol{F}(\boldsymbol{U})=\left(\rho u, \rho u^{2}+p, \rho u v,(E+p) u\right)^{T}, \\
\boldsymbol{G}(\boldsymbol{U})=\left(\rho v, \rho u v, \rho v^{2}+p,(E+p) v\right)^{T} .
\end{gathered}
$$

em que $(u, v)^{T}$ é o vetor velocidade e $\rho v$ é a quantidade de movimento na direção $y$. As demais variáveis já foram definidas anteriormente. Para fechar o sistema, considera-se a equação do gás ideal

$$
p=(\gamma-1)\left(E-\frac{1}{2} \rho\left(u^{2}+v^{2}\right)\right) .
$$

As condições iniciais consideradas são

$$
\rho(x, y, 0)=\rho_{0}(x, y), u(x, y, 0)=u_{0}(x, y), v(x, y, 0)=v_{0}(x, y) \text {, e } p(x, y, 0)=p_{0}(x, y) \text {. }
$$

Este sistema hiperbólico é suplementado com extrapolação de ordem zero no contorno (ver LeVeque (2004) [41]).

\subsubsection{Equações de águas rasas}

As equações de águas rasas 2D formam um conjunto de EDPs que descrevem problemas de fluxo de fluidos em canais abertos. Elas são derivadas das leis de conservação do massa e do momento, além disso são validas para problemas em que as dimensões verticais podem ser desconsideradas quando comparadas as dimensões horizontais. Estas equações são da forma

$$
\frac{\partial \boldsymbol{U}}{\partial t}+\frac{\partial \boldsymbol{F}(\boldsymbol{U})}{\partial x}+\frac{\partial \boldsymbol{G}(\boldsymbol{U})}{\partial y}=\boldsymbol{S}, \quad(x, y) \in\left[x_{L}, x_{R}\right] \times\left[y_{B}, y_{T}\right], \quad t \geq 0,
$$

onde o vetor de quantidades conservadas $\boldsymbol{U}$ e vetores fluxos $\boldsymbol{F}(\boldsymbol{U})$ e $\boldsymbol{G}(\boldsymbol{U})$ são dadas, respec- 
tivamente, como

$$
\begin{gathered}
\boldsymbol{U}=(h, h u, h v)^{T}, \\
\boldsymbol{F}(\boldsymbol{U})=\left(h u, h u^{2}+\frac{1}{2} g h^{2}, h u v\right)^{T}, \\
\boldsymbol{G}(\boldsymbol{U})=\left(h v, h u v, h v^{2}+\frac{1}{2} g h^{2}\right)^{T},
\end{gathered}
$$

onde o vetor $(h u, h v)^{T}$ é a vazão, sendo $h$ a altura da camada de fluido e $g=9.8 \mathrm{~m} / \mathrm{s}^{2}$ a constante gravitacional. Nesse estudo consideram-se as condições iniciais da forma

$$
u(x, y, 0)=u_{0}(x, y), \quad v(x, y, 0)=v_{0}(x, y) \quad \text { e } \quad h(x, y, 0)=h_{0}(x, y)
$$

e as condições de contorno adotadas foram extrapolação de ordem zero (ver LeVeque (2004) [41]).

\subsection{Equações de Navier-Stokes}

As equações instantâneas de Navier-Stokes formam um sistema de EDPs não-lineares que descrevem o movimento de fluidos; em particular de escoamentos incompressíveis com superfícies livres móveis no regime laminar, objeto desse estudo. Este sistema de equações é constituído de uma equação de conservação de massa (continuidade) e três equações da quantidade de movimento, e são restritas a meios contínuos e fluidos Newtonianos. Estas equações são dadas por

$$
\begin{aligned}
\frac{\partial \mathbf{v}}{\partial t}+\nabla \cdot(\mathbf{v v}) & =-\nabla p+\frac{1}{R e} \nabla^{2} \mathbf{v}+\frac{1}{F_{r}^{2}} \mathbf{g}, \quad \text { para } \quad \mathbf{x} \in \Omega, \\
\nabla \cdot \mathbf{v} & =0
\end{aligned}
$$

onde $\mathbf{x}$ é o vetor posição, $t$ é o tempo, v é o campo de velocidade, Re é o número de Reynolds definido como $R e=L_{0} U_{0} / \nu$ e $F_{r}=U_{0} / \sqrt{L_{0}|\mathbf{g}|}$ é o número de Froude, sendo $U_{0}$ e $L_{0}$ as escalas de velocidade e comprimento característicos, respectivamente. $\nu=\frac{\mu}{\rho}$ ( $\mu$ é a viscosidade dinâmica e $\rho$ a massa específica) e $\mathbf{g}$ é o vetor de forçãs externas. Em coordenadas cartesianas e na notação de Einstein, as equações de Navier-Stokes assumem as seguintes formas:

$$
\begin{gathered}
\frac{\partial u_{i}}{\partial t}+\frac{\partial\left(u_{i} u_{j}\right)}{\partial x_{j}}=-\frac{\partial p}{\partial x_{i}}+\frac{1}{R e} \frac{\partial}{\partial x_{j}}\left(\frac{\partial u_{i}}{\partial x_{j}}\right)+\frac{1}{F r^{2}} g_{i}, \quad i=1,2,3 \\
\frac{\partial u_{i}}{\partial x_{i}}=0
\end{gathered}
$$

em que $x_{i}, u_{i}$ e $g_{i}$ são, respectivamente, as coordenadas cartesianas, as componentes do campo de velocidade $\mathbf{v}$ e as componentes do campo gravitacional $\mathbf{g}$. E em coordenadas cilíndricas têm as formas 


$$
\begin{gathered}
\frac{\partial u}{\partial t}+\frac{1}{r} \frac{\partial(r u u)}{\partial r}+\frac{\partial(u v)}{\partial z}=-\frac{\partial p}{\partial r}+\frac{1}{R e} \frac{\partial}{\partial z}\left(\frac{\partial u}{\partial z}-\frac{\partial v}{\partial r}\right)+\frac{1}{F r^{2}} g_{r} \\
\frac{\partial v}{\partial t}+\frac{1}{r} \frac{\partial(r v u)}{\partial r}+\frac{\partial(v v)}{\partial z}=-\frac{\partial p}{\partial z}+\frac{1}{R e} \frac{1}{r} \frac{\partial}{\partial r}\left[r\left(\frac{\partial u}{\partial z}-\frac{\partial v}{\partial r}\right)\right]+\frac{1}{F r^{2}} g_{z} \\
\frac{1}{r} \frac{\partial(r u)}{\partial r}+\frac{\partial v}{\partial z}=0
\end{gathered}
$$

em que $u=u(r, z, t)$ e $v=v(r, z, t)$ são os componentes do vetor velocidade nas direções $r$ e $z$ respectivamente e $g=\left(g_{r}, g_{z}\right)^{T}$ representa o campo gravitacional.

As equações de Navier-Stokes requerem condições iniciais e de contorno fisicamente apropriadas para definir problemas específicos. Essas condições são impostas principalmente para se obter uma solução numérica de boa qualidade. Para as condições iniciais, deve-se conhecer, em $t=0$, a distribução espacial das variáveis dependentes. Para as condições de contorno prescreve-se alguma informação física, para todo tempo $t$, das variáveis dependentes no contorno da região, limitando o movimento do fluido. Em síntese, se $\Omega$ é um domínio limitado no $\mathbb{R}^{N}, N=2,3, \partial \Omega$ é a fronteira limitando $\Omega$, a e $\mathbf{b}$ são, respectivamente, funções conhecidas sobre $\Omega$ e $\partial \Omega$. As condições iniciais e de fronteira típicas para (2.28)-(2.29) ou para (2.32)-(2.34) têm as seguintes formas:

$$
\begin{gathered}
\mathbf{v}(\mathbf{x}, 0)=\mathbf{a}(\mathbf{x}), \quad \mathbf{x} \in \Omega, \\
\mathbf{v}(\mathbf{x}, t)=\mathbf{b}(\mathbf{x}, t), \quad \mathbf{x} \in \partial \Omega, \quad t>0,
\end{gathered}
$$

com a e b satisfazendo

$$
\nabla \cdot \mathbf{a}=0, \quad \int_{\partial \Omega}(\mathbf{b} \cdot \mathbf{n}) d \mathbf{A}=0, \quad \mathbf{a}(\mathbf{x})=\mathbf{b}(\mathbf{x}, 0)
$$

em que $\mathbf{n}$ é o vetor normal exterior à $\partial \Omega$.

Nos problemas de interesse neste trabalho, as condições de contorno mais usuais são como segue:

-Entrada do fluido (injetor):

$$
u_{n}=U_{0}, \quad u_{t}=0
$$

onde $u_{n}$ é a velocidade normal ao contorno e $u_{t}$ é a velocidade tangencial a ele. 
-Saída do fluido (ejetor):

$$
\frac{\partial u_{n}}{\partial \mathbf{n}}=\frac{\partial u_{t}}{\partial \mathbf{n}}=0
$$

-Contorno rígido:

- Free-slip: (condição com escorregamento),

$$
u_{n}=0 \quad \text { e } \quad \frac{\partial u_{t}}{\partial \mathbf{n}}=0
$$

- No-slip: (condição sem escorregamento),

$$
u_{n}=0 \quad \text { e } \quad u_{t}=0 .
$$

- Superfície livre:

$$
\begin{aligned}
& \mathbf{n} \cdot(\sigma \cdot \mathbf{n})=p_{\text {ext }}, \\
& m_{1} \cdot(\sigma \cdot \mathbf{n})=0 \\
& m_{2} \cdot(\sigma \cdot \mathbf{n})=0
\end{aligned}
$$

onde $P_{\text {ext }}$ é a pressão externa (atmosférica), a qual neste trabalho foi considerada como zero. Para o caso $3 \mathrm{D}, m_{1}=\left(m_{1 x}, m_{1 y}, m_{1 z}\right)$ e $m_{2}=\left(m_{2 x}, m_{2 y}, m_{2 z}\right)$ são os vetores tangentes à superfície livre e $\mathbf{n}=\left(n_{x}, n_{y}, n_{z}\right)$; para o caso $2 \mathrm{D}$ esses vetores reduzem-se a $m_{1}=\left(m_{1 x}, m_{1 y}\right)$. $m_{2}=0$ e $\mathbf{n}=\left(n_{x}, n_{y}\right)$. Para o caso axissimétrico considera-se $m_{1}=\left(-n_{z}, 0, n_{r}\right)$ e $\mathbf{n}=$ $\left(n_{r}, 0, n_{z}\right)$. O tensor de tensões total $\sigma$ é dado por

$$
\sigma=-p I+\frac{1}{R_{e}}\left(\nabla u+(\nabla u)^{T}\right)
$$

onde $I$ é o tensor identidade.

\subsection{Estimativa de Erro}

Com a finalidade de verificar a precisão local dos esquemas numéricos estudados neste trabalho faz-se necessario determinar a ordem (local) de convergência. Para tanto calculam-se os erros relativos nas normas $L_{1}, L_{2}$ e $L_{\infty}$ respectivamente como:

$$
\left\|E_{h}\right\|_{1}=\frac{\sum_{i=1}^{N}\left|u_{i, \text { exata }}-u_{i, \text { num }}\right|}{\sum_{i=1}^{N}\left|u_{i, \text { exata }}\right|}
$$




$$
\left\|E_{h}\right\|_{2}=\sqrt{\frac{\sum_{i=1}^{N}\left(u_{i, \text { exata }}-u_{i, \text { num }}\right)^{2}}{\sum_{i=1}^{N}\left(u_{i, \text { exata }}\right)^{2}}},
$$

onde $N$ representa o número de pontos da malha, $u_{i, \text { exata }}$ e $u_{i, n u m}$ são as soluções exata e numérica, respectivamente, para cada ponto da malha. Uma estimativa para a precisão local $q$ de um método numérico é obtida admitindo-se que a solução exata é conhecida em cada ponto de uma malha de tamanho $h$ e o erro na norma $k, k=1,2$, tem o seguinte comportamento

$$
\begin{gathered}
\left\|E_{h}\right\|_{k} \approx C h^{q} \text { para } h \rightarrow 0 \text { ou na escala log-log } \\
\log \left(\left\|E_{h}\right\|_{k}\right) \approx \log C+q \log h,
\end{gathered}
$$

onde $C$ é a constante do erro. Ao se refinar a malha usando o tamanho do espaçamento como $\frac{h}{2}$ obtém-se

$$
\left\|E_{\frac{h}{2}}\right\|_{k} \approx C\left(\frac{h}{2}\right)^{q}
$$

Dividindo (2.48) por (2.50) resulta em

$$
\frac{\left\|E_{h}\right\|_{k}}{\left\|E_{\frac{h}{2}}\right\|_{k}} \approx 2^{q} .
$$

E uma estimativa para $q$ (a ordem de convergência) é obtida de (2.51) como

$$
q \approx \frac{\log \left(\frac{\left\|E_{h}\right\|_{k}}{\left\|E_{\frac{h}{2}}\right\|_{k}}\right)}{\log 2} .
$$

Pode-se encontrar mais detalhes a respeito de estimativas de erro em Rider (2007) [56]. 


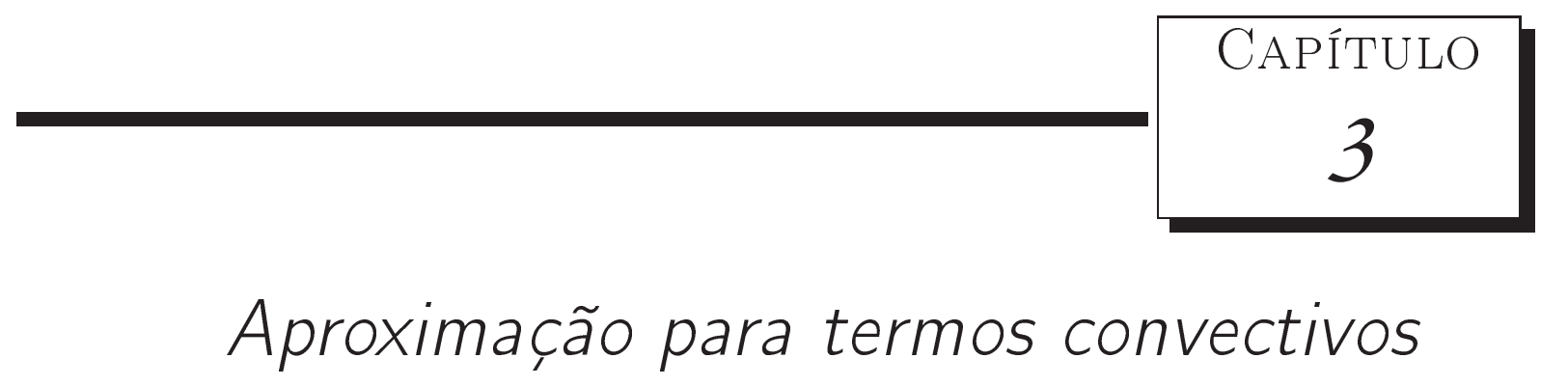

O objetivo deste capítulo é apresentar os conceitos fundamentais para a derivação de esquemas upwind de alta resolução, os quais são aproximações para os termos convectivos (lineares ou não lineares). Em particular, são utilizados o conceito de Variáveis Normalizadas (VN) proposto por Leonard (1988) [37], as restrições TVD (Total Variation Diminishing) introduzidas por Harten (1983) [30], o critério de limitação CBC (Convection Boundedness Criterion) de Gaskell e Lau (1988) [23] e o critério de limitação mais flexível de Spekreijse (1987) [66]. Além disso, é descrita uma metodologia de derivação dos limitadores de fluxo correspondentes aos esquemas utilizados/derivados neste trabalho.

\subsection{Aproximação dos Termos Convectivos}

Grandes esforços da comunidade científica em CFD têm sido direcionados no sentido de desenvolver esquemas upwind de alta resolução (maior ou igual a 2) que sejam capazes de corrigir alguns inconvenientes, tais como oscilações não físicas e suavização, que surgem no cálculo numérico da solução. Em particular, na discretização das derivadas convectivas das equações de conservação é crucial. Nesta seção é apresentada a estratégia upwind para aproximar termos convectivos (em geral não lineares). Para tanto, são utilizadas as seguintes notações a seguir para as posições dos nós na malha computacional, os quais são definidos conforme o sinal da velocidade convectiva numa face do volume de controle. A Figura 3.1 ilustra o caso 1D de uma propriedade transportada $\phi$ com velocidade $V_{f}$ atraves de uma face $f$ do volume de controle; onde, nesta Figura, o nó a jusante de $f$ é nomeado como $D$ (o Downstream), o nó a montante como $U$ (o Upstream), e a posição mais a montante é nomeada como $R$ (o Remote-upstream). O fluxo numérico, $\phi_{f}$, na face $f$ (ou o esquema upwind) é então definido pela relação funcional 

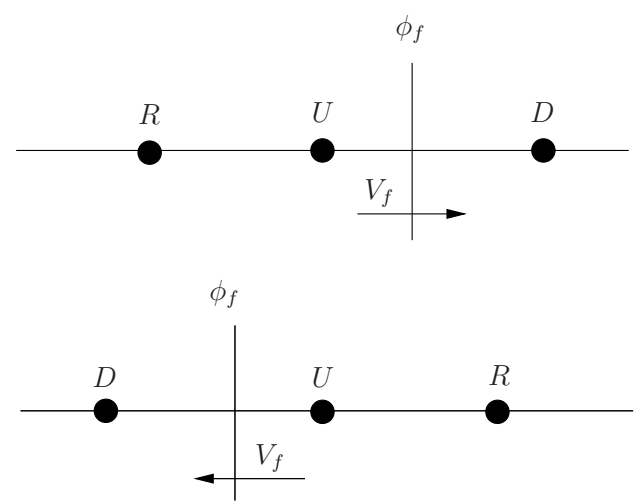

Figura 3.1: Localização dos nós computacionais $D, U$ e $R$ em função da velocidade convectiva $V_{f}$ (fluxo) de uma variável convectada $\phi$.

$$
\phi_{f}=\phi_{f}\left(\phi_{D}, \phi_{U}, \phi_{R}\right)
$$

\subsection{Variáveis normalizadas}

Criar métodos numéricos que sejam capazes de resolver gradientes elevados e, ao mesmo tempo, manter a estabilidade das soluções computacionais é uns dos grandes desafios na análise numérica. Tentando alcançar essas metas, Leonard, Gaskell e Lau propuseram respectivamente o conceito de variáveis normalizadas (1988) [37] e o critério de limitação CBC (1988) [23].

Para simplificar a relação funcional (3.1), a variável normalizada de Leonard (1988) [37] é utilizada com frequência. Esta variável é definida como

$$
\hat{\phi}_{()}=\frac{\phi_{()}-\phi_{R}}{\phi_{D}-\phi_{R}}
$$

em que $\phi_{D}$ e $\phi_{R}$ são os valores não-normalizados da propriedade $\phi$ nos pontos $D$ e $R$, respectivamente. Pode-se notar que com esta normalização, $\hat{\phi}_{R}=0$ e $\hat{\phi}_{D}=1$. Além disso, $\phi_{U}=\phi_{R}$ se $\hat{\phi}_{U}=0$ e $\phi_{U}=\phi_{D}$ se $\hat{\phi}_{U}=1$. Então qualquer esquema de convecção que utilize somente os valores de $\phi$ nos pontos $D, U, R$ para avaliar o fluxo numérico $\phi_{f}$, pode ser representado da forma funcional simplificada

$$
\hat{\phi}_{f}=\hat{\phi}_{f}\left(\hat{\phi}_{U}\right)
$$

Pode-se observar que o uso de variáveis normalizadas simplifica a definição da relação funcional de esquemas de alta resolução (3.1) e facilita a derivação das condições que esta relação deve satisfazer para se obter soluções limitadas (ver por exemplo M. Darwish and F. Moukalled (2003) [16]). Por exemplo, o esquema QUICK (Quadratic Upstream Interpolation for Convective 
Kinematics, ver Leonard (1979) [36]), é dado por

$$
\phi_{f}=\phi_{f}\left(\phi_{D}, \phi_{U}, \phi_{R}\right)=\frac{1}{2}\left(\phi_{D}+\phi_{U}\right)-\frac{1}{8}\left(\phi_{R}-2 \phi_{U}+\phi_{D}\right)
$$

E quando se usa a relação (3.2), o esquema QUICK torna-se

$$
\hat{\phi}_{f}=\hat{\phi}_{f}\left(\hat{\phi}_{U}\right)=\frac{3}{8}+\frac{3}{4} \hat{\phi}_{U}
$$

A relação funcional (3.3) pode ser representada em um diagrama de variáveis normalizadas, isto é, plotando-a no plano $\hat{\phi}_{U} \perp \hat{\phi}_{f}$ como pode-se observar na Figura 3.2. Nesta Figura mostra-se este diagrama para alguns esquemas em variáveis normalizadas, como por exemplo QUICK, Diferença Central e FOU (First Order Upwind).

O diagrama de variáveis normalizadas é uma ferramenta bastante útil para se ter uma ideia geral da precisão e da difusividade dos esquemas de alta resolução. Por exemplo, Leonard (1988) [37] mostrou por meio deste diagrama que para se derivar esquemas monotônicos de alta ordem não-lineares (ou lineares por partes), formulados em variáveis normalizadas com $0 \leq \hat{\phi}_{U} \leq 1$, estes tem que satisfazer as seguintes condições: passar pelos pontos $O(0,0)$ e $P(1,1)$ (para ser monotônico), passar pelo ponto $Q(0.5,0.75)$ (para possuir segunda ordem de precisão) e, ainda mais, se a inclinação dessa relação no ponto $Q$ é 0.75 (derivada de $\hat{\phi}_{f}$ ), então o esquema é de terceira ordem (QUICK Leonard (1979) [36] por exemplo). Além disso, Leonard recomenda que para $\hat{\phi}_{U} \notin[0,1]$ seja adotado o esquema FOU.

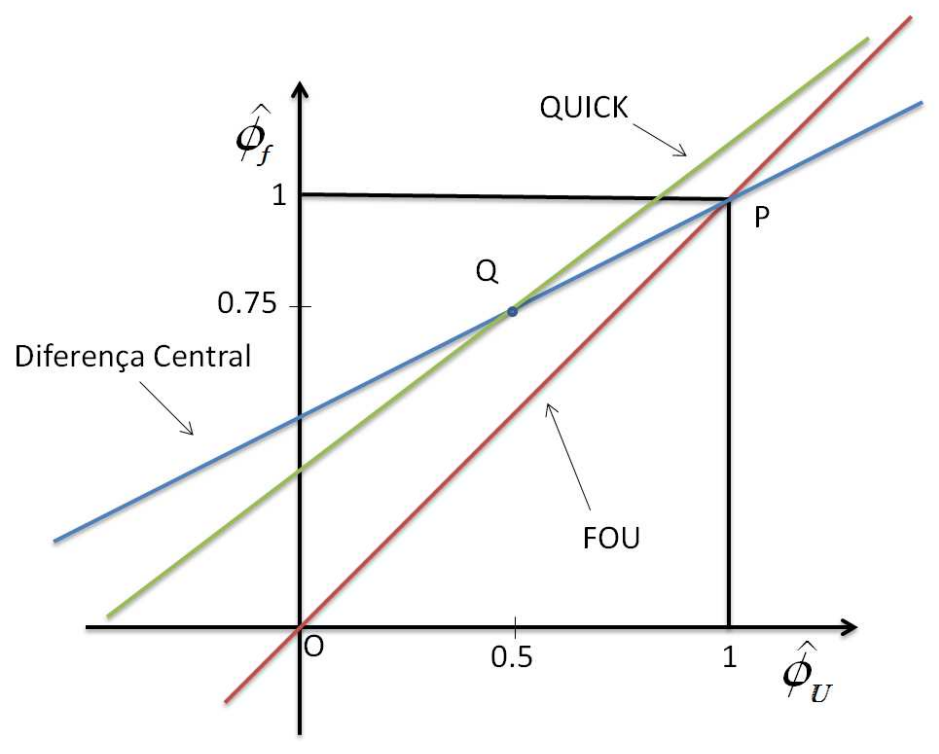

Figura 3.2: Diagrama de variáveis normalizadas mostrando os esquemas QUICK, FOU e Diferença Central. 


\subsection{Esquemas TVD}

Esquemas satisfazendo as condições TVD foram desenvolvidos no princípio para resolver problemas em dinâmica de gases, especialmente problemas de Riemann. As bases desses esquemas foram estabelecidas a partir das ideias de Godunov que desenvolveu um esquema para sistemas hiperbólicos não-lineares de leis de conservação. Esquemas de advecção tem recebido, na atualidade, extensiva atenção dos pesquisadores, principalmente no caso de problemas de escoamentos de fluidos em altas velocidades envolvendo choques. Devido a isso, uma série de esquemas tem sido desenvolvidos combinando alta ordem de precisão local e soluções fisicamente permissíveis. Para a construção de esquemas deste tipo é fundamental o uso do conceito TVD introduzido por Harten (1983) [30]. Apresenta-se a seguir as noções fundamentais para se derivar esquemas TVD.

A principal motivação para o estudo de esquemas TVD é a necessidade de estratégias de alta ordem que, a cada passo de tempo, evitem o aparecimento de novos extremos (máximos ou mínimos) na solução numérica. Considere uma sequência de aproximações discretas

$$
\phi(t)=\left\{\phi_{i}(t)\right\}_{i \in \mathbb{Z}}
$$

A variação total TV (Total Variation) da solução discreta no tempo $t$ é definida por

$$
T V(\phi(t))=\sum_{i \in \mathbb{Z}}\left|\phi_{i+1}(t)-\phi_{i}(t)\right|
$$

Considera-se um método de diferenças explícito envolvendo $(2 k+1)$ pontos da forma

$$
\phi_{i}^{n+1}=H\left(\phi_{i-k}^{n}, \ldots, \phi_{i+k}^{n}\right), \quad \forall n \geq 0, i \in \mathbb{Z},
$$

onde $H: \mathbb{R}^{2 k+1} \longrightarrow \mathbb{R}$ é uma função contínua e $\phi_{i}^{n}$ é uma aproximação da solução exata $\phi$ nos pontos de malha uniforme $\left(x_{i}, t_{n}\right)$, com $x_{i}=i \delta x, t_{n}=n \delta t$, sendo $\delta x$ e $\delta t$ os espaçamentos espaciaes e temporaes respectivamente. O esquema (3.8) é dito ser TVD se

$$
T V\left(\phi^{n+1}\right) \leq T V\left(\phi^{n}\right)
$$

A propriedade TVD é desejável porque ela garante que oscilações não físicas não podem aparecer na solução numérica, isto é, um esquema é monotônico (ou preserva monotonicidade) se dado que $\phi_{i}^{n} \geq \phi_{i+1}^{n}$, para todo $i$ implica que $\phi_{i}^{n+1} \geq \phi_{i+1}^{n+1}$. 
Apresenta-se a seguir um critério bastante útil, conhecido como lema de Harten (ver Harten (1983) [30]), o qual assegura a propriedade TVD para um esquema numérico.

Considera-se um método numérico na forma

$$
\phi_{i}^{n+1}=\phi_{i}^{n}+C_{i+1 / 2}\left(\phi_{i+1}^{n}-\phi_{i}^{n}\right)-D_{i-1 / 2}\left(\phi_{i}^{n}-\phi_{i-1}^{n}\right),
$$

em que os coeficientes $D_{i-1 / 2}, C_{i+1 / 2}$ são funções dos $\phi_{i}^{n}$ envolvidos. Se os coeficientes em (3.10) satisfazem as seguintes desigualdades para todo $i$

$$
\begin{aligned}
C_{i+1 / 2} & \geq 0, \\
D_{i+1 / 2} & \geq 0, \\
0 \leq C_{i+1 / 2}+D_{i+1 / 2} & \leq 1,
\end{aligned}
$$

então o esquema é TVD.

\subsection{Critério de limitação CBC}

Obter soluções numéricas limitadas com o uso de um esquema de convecção é de suma importância no transporte de propriedades físicas. Para alcançar esses propósitos, Gaskell e Lau (1988) [23] propuseram o critério de limitação CBC. O critério assegura que se a relação funcional $\hat{\phi}_{f}=\hat{\phi}_{f}\left(\hat{\phi}_{U}\right)$ é crescente e contínua (ou crescente e contínua por partes), então a solução é limitada, se satisfaz as condições

$$
\left\{\begin{array}{l}
\hat{\phi}_{U} \leq \hat{\phi}_{f}\left(\hat{\phi}_{U}\right) \leq 1, \quad \text { se } \quad \hat{\phi}_{U} \in[0,1] \\
\hat{\phi}_{f}(0)=0, \\
\hat{\phi}_{f}(1)=1, \\
\hat{\phi}_{f}\left(\hat{\phi}_{U}\right)=\hat{\phi}_{U} \quad \text { se } \quad \hat{\phi}_{U} \notin[0,1] .
\end{array}\right.
$$

Fundamentalmente, o papel do critério CBC é manter a solução numérica dentro das regiões estáveis. Essas condições são ilustradas na Figura 3.3.

\subsection{Limitadores de fluxo}

Associado aos esquemas TVD está o conceito muito importante de limitadores de fluxo. O conceito foi introduzido por van Leer $(1973,1974)$ [76, 77] e Boris e Book $(1973,1976)[5,6]$. A ação de um limitador de fluxo é diminuir a importância do termo antidifusivo próximo às descontinuidades; há também a necessidade de se detectar a localização das descontinuidades. 


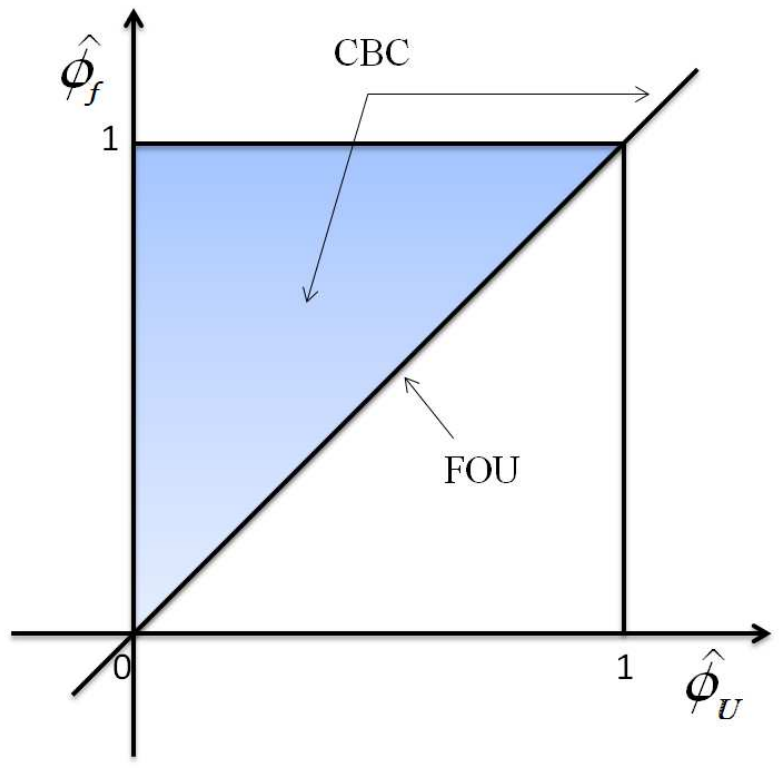

Figura 3.3: Diagrama CBC em variáveis normalizadas

Isso pode ser feito analisando-se os gradientes consecutivos avaliados nas faces (digamos $f$ e g) do volume de controle. Um limitador de fluxo pode ser obtido reescrevendo-se um esquema numérico em variáveis não-normalizadas. Por exemplo, o esquema numérico adotado com frequência na literatura (ver Waterson e Deconink (2007) [81]) é da forma

$$
\phi_{f}=\phi_{U}+\frac{1}{2} \varphi\left(r_{f}\right)\left(\phi_{D}-\phi_{U}\right)
$$

em que $\varphi\left(r_{f}\right)=\varphi_{f}$ é o limitador do fluxo com $r_{f}$ sendo a razão de gradientes consecutivos dada por

$$
r_{f}=\frac{\left(\frac{\partial \phi}{\partial x}\right)_{g}}{\left(\frac{\partial \phi}{\partial x}\right)_{f}} \equiv \frac{b}{a}
$$

a qual, para malhas uniformes, pode ser rescrita como

$$
r_{f}=\frac{\phi_{U}-\phi_{R}}{\phi_{D}-\phi_{U}}
$$

e em termos de variáveis normalizadas como 


$$
r_{f}=\frac{\hat{\phi}_{U}}{1-\hat{\phi}_{U}} .
$$

Com estas considerações em mente, Sweby (1984) [67] deduziu condições suficientes para que um esquema seja TVD, isto é

$$
\begin{cases}0 \leq \varphi\left(r_{f}\right) \leq \min 2 r_{f}, 2 & \text { se } \quad r_{f} \geq 0 \\ \varphi\left(r_{f}\right)=0 & \text { se } \quad r_{f} \leq 0\end{cases}
$$

Geometricamente, a condição (3.16) diz que o gráfico de $\varphi$ deve estar contido na região hachurada da Figura 3.4 (região TVD).

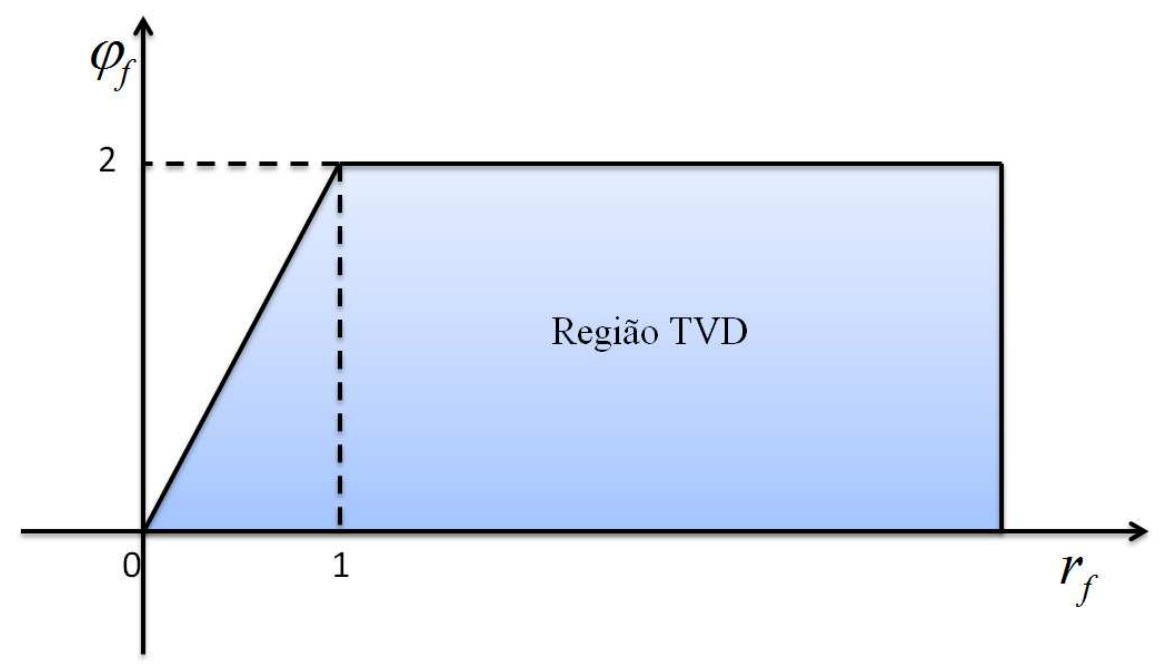

Figura 3.4: Diagrama de limitador de fluxo - Diagrama de Sweby.

De acordo com Waterson e Deconinck (2007) [81], para preservar linearidade e segunda ordem de precisão numa malha uniforme o limitador de fluxo $\varphi$ deve satisfazer a propriedade

$$
\varphi(1)=1
$$

Além disso, para o limitador atingir terceira ordem de precisão, Zijlema (1996) [85] propôs a seguinte condição necessária e suficiente

$$
\varphi^{\prime}(1)=1 / 4 .
$$

Ainda mais, foi tomado em conta, uma propriedade proposta por Sweby (1984) [67], a chamada de princípio de monotonicidade, dada por

$$
r_{f} \longrightarrow 0 \Rightarrow \varphi^{\prime}\left(r_{f}\right)=2 .
$$


Finalmente, para que os esquemas sejam TVD, Sweby (1984) [67] utilizou as ideias de Harten (1983) [30], dando origem a uma série de restrições no contexto de variáveis normalizadas, a saber

$$
\begin{cases}\hat{\phi}_{U} \leq \hat{\phi}_{f} \leq \min \left\{1,2 \hat{\phi}_{U}\right\} & \text { se } 0<\hat{\phi}_{U}<1 \\ \hat{\phi}_{f}=\hat{\phi}_{U}, & \text { se } \quad \hat{\phi}_{U} \leq 0 \quad \text { ou } \quad \hat{\phi}_{U} \geq 1 .\end{cases}
$$

A Figura 3.5 ilustra a região TVD de Sweby.

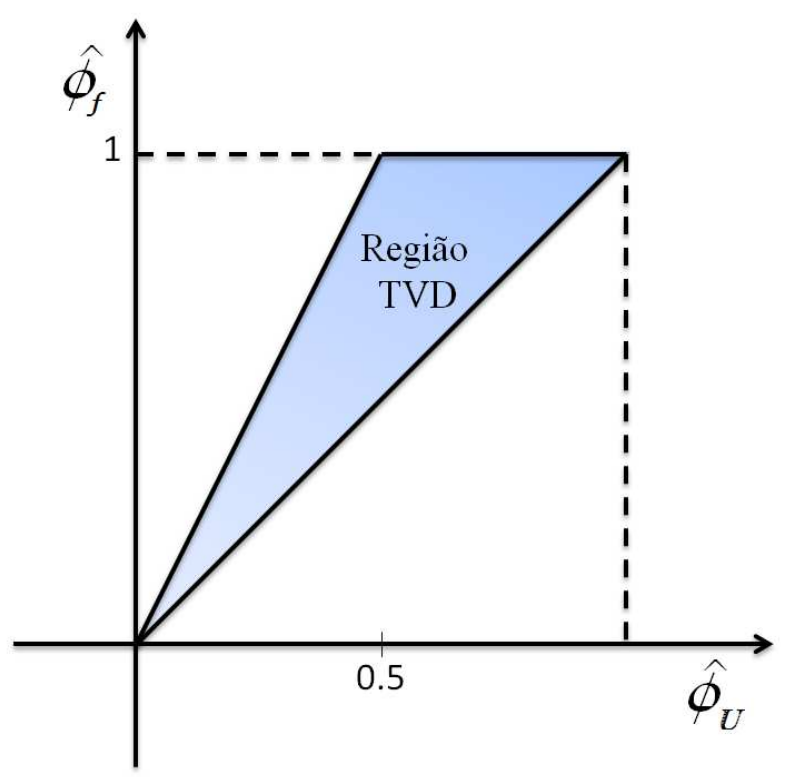

Figura 3.5: Região TVD em Variáveis Normalizadas.

Há também um critério de limitação mais flexível devido a Spekreijse (1987) [66] que pode ser usado para derivar limitadores de fluxo que dão origem a métodos upwind monotônicos. Em resumo, as condições de Spekreijse sobre a função limitadora $\varphi$ para se obter métodos numéricos de segunda ordem de precisão e preservar a monotonicidade são dadas por

$$
\begin{gathered}
\alpha \leq \varphi\left(r_{f}\right) \leq M, \quad \forall r_{f}, \\
-M \leq \frac{\varphi\left(r_{f}\right)}{r_{f}} \leq 2+\alpha \quad \forall r_{f} .
\end{gathered}
$$

A região de monoticidade de Spekreijse formalizada nas condições (3.21)-(3.22) está ilustrada na Figura 3.6, onde $\alpha$ e $M$ são constantes satisfazendo: $\alpha \in[-2,0]$ e $M \in[0, \infty]$.

A região de Sweby no diagrama de limitadores de fluxo está inteiramente contida na região de monotonicidades de Spekreijse, para $\alpha=0$ e $M=2$; e portanto todo esquema TVD satisfaz as restrições (3.21)-(3.22). 


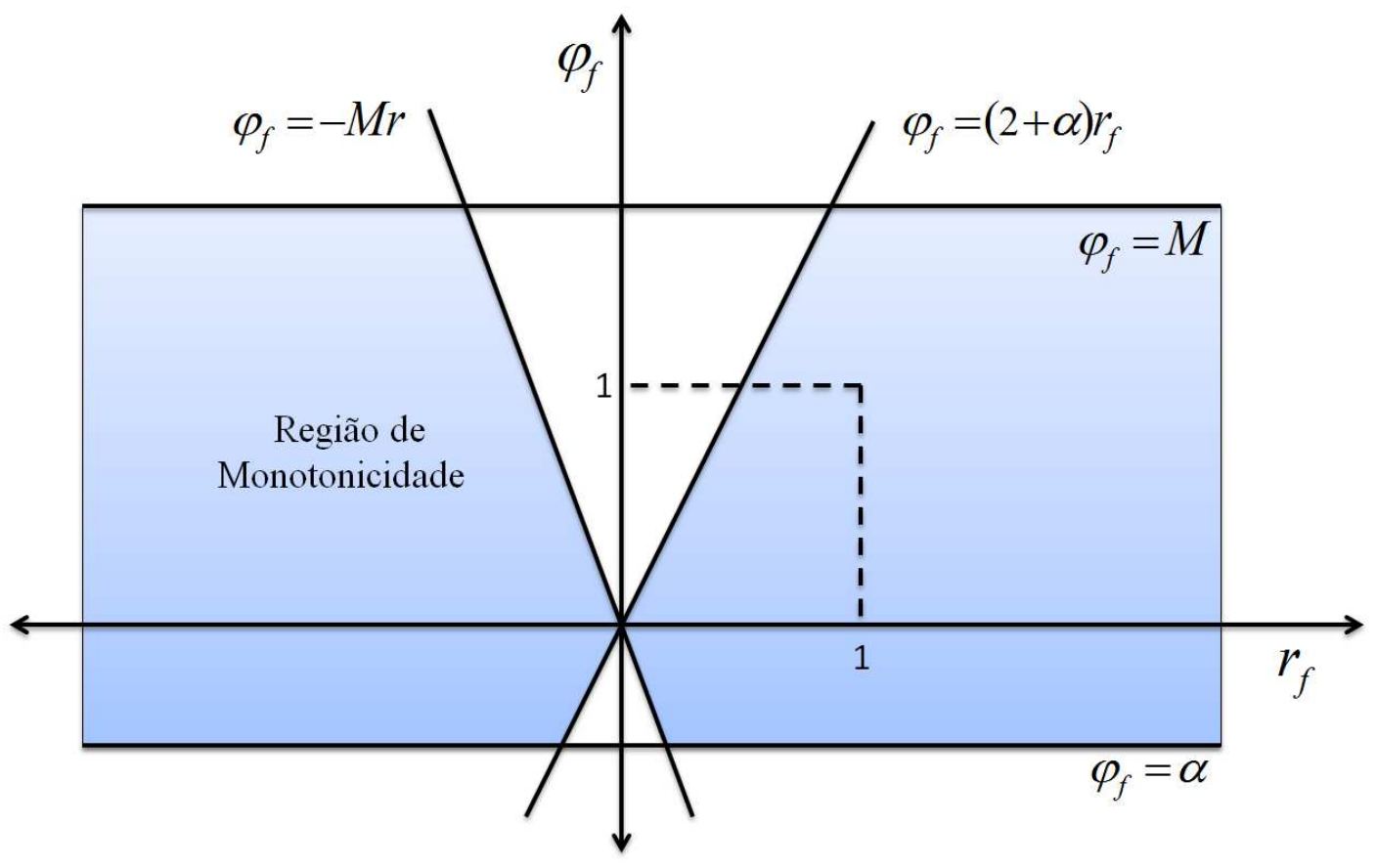

Figura 3.6: Região de monoticidade de Spekreijse.

\subsection{Exemplos de esquemas convectivos}

Nesta seção são apresentados de maneira resumida alguns esquemas consolidados na literatura e seus respectivos limitadores de fluxos. Esses esquemas foram objeto de nosso estudo com a finalidade de confrontar resultados obtidos com eles com os resultados obtidos pelos esquemas aqui propostos.

\subsection{1 van Albada (satisfaz as restrições de Spekreijse)}

Esse esquema foi proposto por van Albada (1982) [75] em variáveis normalizadas é dado por

$$
\hat{\phi}_{f}=\frac{\hat{\phi}_{U}\left(4 \hat{\phi}_{U}^{2}-5 \hat{\phi}_{U}+3\right)}{4 \hat{\phi}_{U}^{2}-4 \hat{\phi}_{U}+2}
$$

e correspondente limitador de fluxo por

$$
\varphi\left(r_{f}\right)=\frac{r_{f}\left(r_{f}+1\right)}{r_{f}^{2}+1}, \quad \forall r_{f}
$$

\subsubsection{MC (TVD)}

O esquema MC (Monotonized Central-difference) foi proposto por van Leer $(1974,1977)$ $[77,78]$, que pode ser escrito em variáveis normalizadas como 


$$
\hat{\phi}_{f}= \begin{cases}2 \hat{\phi}_{U}, & \text { se } 0 \leq \hat{\phi}_{U}<1 / 2, \\ \hat{\phi}_{U}+\frac{1}{4}, & \text { se } 1 / 2<\hat{\phi}_{U} \leq 3 / 4, \\ 1, & \text { se } 3 / 4<\hat{\phi}_{U} \leq 1 \\ \hat{\phi}_{U}, & \text { se } \quad \hat{\phi}_{U} \notin[0,1]\end{cases}
$$

com limitador de fluxo dado pela expressão

$$
\varphi\left(r_{f}\right)=\max \left\{0, \min \left[2 r_{f}, \frac{1+r_{f}}{2}, 2\right]\right\} .
$$

\subsubsection{Minmod (TVD)}

O esquema Minmod foi proposto por Roe (1982) [61]. Em variáveis normalizadas é dado como

$$
\hat{\phi}_{f}= \begin{cases}\frac{3}{2} \hat{\phi}_{U}, & \text { se } 0 \leq \hat{\phi}_{U}<1 / 2, \\ \frac{1}{2}\left(\hat{\phi}_{U}+1\right), & \text { se } 1 / 2<\hat{\phi}_{U} \leq 1, \\ \hat{\phi}_{U}, & \text { se } \hat{\phi}_{U} \notin[0,1],\end{cases}
$$

com limitador de fluxo

$$
\varphi\left(r_{f}\right)=\max \left\{0, \min \left(r_{f}, 1\right)\right\}
$$

\subsubsection{Superbee (TVD)}

O esquema Superbee foi desenvolvido por Roe $(1985,1986)[58,59]$ e é dado em variáveis normalizadas como

$$
\hat{\phi}_{f}=\left\{\begin{array}{lc}
2 \hat{\phi}_{U}, & 0 \leq \hat{\phi}_{U}<1 / 3, \\
\frac{1}{2}\left(\hat{\phi}_{U}+1\right), & 1 / 3<\hat{\phi}_{U} \leq 1 / 2, \\
\frac{3}{2} \hat{\phi}_{U}, & 1 / 2<\hat{\phi}_{U} \leq 2 / 3, \\
1, & 2 / 3<\hat{\phi}_{U} \leq 1, \\
\hat{\phi}_{U}, & \hat{\phi}_{U} \notin[0,1],
\end{array}\right.
$$

com limitador de fluxo

$$
\varphi\left(r_{f}\right)=\max \left[0, \min \left(2 r_{f}, 1\right), \min \left(r_{f}, 2\right)\right\}
$$

\subsubsection{ADBQUICKEST (TVD)}

O esquema ADBQUICKEST (ADaptative Bounded Quadratic Upstream for Convective Kinematics with Estimated Streaming Terms) foi desenvolvido por Ferreira et al. (2009) [20]. Em variáveis normalizadas é dado como 


$$
\hat{\phi}_{f}= \begin{cases}(2-\theta) \hat{\phi}_{U}, & \text { se } 0 \leq \hat{\phi}_{U}<a \\ \hat{\phi}_{U}+\frac{1}{2}(1-|\theta|)\left(1-\hat{\phi}_{U}\right)-\frac{1}{6}\left(1-\theta^{2}\right)\left(1-2 \hat{\phi}_{U}\right), & \text { se } a \leq \hat{\phi}_{U} \leq b \\ 1-\theta+\theta \hat{\phi}_{U}, & \text { se } b<\hat{\phi}_{U} \leq 1 \\ \hat{\phi}_{U}, & \text { se } \hat{\phi}_{U} \notin[0,1]\end{cases}
$$

onde $\theta$ é o número de Courant e $a$ e $b$ são dados por

$$
a=\frac{2-|\theta|+\theta^{2}}{7-6 \theta-3|\theta|+2 \theta^{2}} \quad \text { e } \quad b=\frac{-4+6 \theta-3|\theta|+\theta^{2}}{-5+6 \theta-3|\theta|+2 \theta^{2}} .
$$

O limitador de fluxo correspondente é dado como

$$
\varphi\left(r_{f}\right)=\max \left\{0, \min \left[2 r_{f}, \frac{2+\theta^{2}-3 \theta+\left(1-\theta^{2}\right) r_{f}}{3-3 \theta}, 2\right]\right\} .
$$

\subsubsection{TOPUS (TVD)}

O esquema TOPUS (Third-Order Polynomial Upwind Scheme) foi desenvolvido por Queiroz et al. (2010) [52]. O esquema e limitador de fluxo correspondente são dados, respectivamente, como

$$
\hat{\phi}_{f}=\left\{\begin{array}{lll}
\beta \hat{\phi}_{U}^{4}+(-2 \beta+1) \hat{\phi}_{U}^{3}+\left(\frac{5 \beta-10}{4}\right) \hat{\phi}_{U}^{2}+\left(\frac{-\beta+10}{4}\right) \hat{\phi}_{U}, & \text { se } \hat{\phi}_{U} \in[0,1] \\
\hat{\phi}_{U}, & \text { se } \hat{\phi}_{U} \notin[0,1]
\end{array}\right.
$$

e

$$
\varphi\left(r_{f}\right)=\max \left\{0, \frac{0.5\left(\left|r_{f}\right|+r_{f}\right)(-0.5 \beta+1) r_{f}^{2}+(\beta+4) r_{f}+(-0.5 \beta+3)}{\left(1+\left|r_{f}\right|\right)^{3}},\right\}
$$

$\operatorname{com} \beta \in[-2,2]$. Deve-se ressaltar que o TOPUS forneceu os melhores resultados para o caso em que $\beta=2$.

É oportuno informar neste ponto que foram derivados três esquemas para upwinding: os esquemas FDHERPUS, CSPUS e RUS. Os esquemas FDHERPUS e CSPUS são TVD e o esquema RUS (parcialmente) satisfaz as restrições para monoticidade de Spekreijse. 
CAPÍTUlo

\section{Modelagem computacional}

Este capítulo trata da discretização das leis de conservação hiperbólicas e das equações instantâneas de Navier-Stokes (2.28)-(2.29) ambas descritas no capítulo 2 deste trabalho, incluindo o tratamento dos termos convectivos (lineares e não lineares). Será apresentada também uma descrição da metodologia numérica (algoritmo computacional) utilizada para simular escoamentos incompressíveis com superfícies livres móveis a altos e a baixos números de Reynolds.

\subsection{Discretização das leis de conservação 1D}

Introduzem-se os pontos de malha $x_{i}=i \delta x$ para a discretização do domínio espacial, com tamanho de passo uniforme $\delta x=x_{i+\frac{1}{2}}-x_{i-\frac{1}{2}}$. O tempo também é (uniformemente) discretizado com nível de tempo $t_{n}=n \delta t$, onde $\delta t=t_{n+1}-t_{n}$ representa o tamanho de passo temporal.

\subsubsection{Equação de advecção 1D}

Na equação linear de advecção $(2.3), u=u(x, t)$ é a variavel dependente e $a$ é a velocidade convectiva (constante). Uma solução numérica para esta equação pode ser aproximada pelo método conservativo de diferenças finitas

$$
u_{i}^{n+1}=u_{i}^{n}-C\left(u_{i+\frac{1}{2}}^{n}-u_{i-\frac{1}{2}}^{n}\right),
$$

em que a marcha no tempo é aproximada pelo método de Euler explícito, $u_{i}^{n}$ é a solução numérica nos pontos de malha $\left(x_{i}, t_{n}\right) \equiv(i \delta x, n \delta t) \equiv(i, n)$ e $C=a \frac{\delta t}{\delta x}$ é o número de Courant. Na equação (4.1), $u_{i+1 / 2}^{n}$ e $u_{i-1 / 2}^{n}$, denotados por $u_{f}^{n}$ e $u_{g}^{n}$ respectivamente (ver Figura 4.1), são aproximações para a variavel convectiva $u$ que serão calculadas de acordo com o sinal da velocidade de advecção local $V_{(\cdot)}$ e em função dos valores de $u$ nas posições a montante $U$, mais a montante $R$ e ajusante $D$. Por exemplo, na figura 4.1 para a face $f=i+1 / 2$ as posições $D, U$ e $R$ são, 
respectivamente, $D=i+1, U=i$ e $R=i-1$. Para simplificar, a partir de agora o índice $n$ será (ou não) omitido.

Em síntese, a soluçaõ é aproximada pelo método (4.1) onde a variavel convectada $u$ nas faces $i-1 / 2$ e $i+1 / 2$ é calculada como

$$
u_{i \pm 1 / 2}=\left\{\begin{array}{lll}
u_{R}+\left(u_{D}-u_{R}\right) E U A R, & \text { se } & \hat{u}_{U} \in[0,1] \\
u_{U}, & \text { se } & \hat{u}_{U} \notin[0,1]
\end{array}\right.
$$

onde EUAR (Esquema Upwind de Alta Resolução) representa um esquema upwind tal como (5.12), (5.43) ou (5.53).

O método explícito (4.1) foi implementado utilizando um código computacional próprio. A fim de garantir estabilidade, o passo de tempo é selecionado (automaticamente) de tal maneira que o parâmetro $C$ satisfaz $C \leq 1$. Aqui $C$ denota a velocidade máxima de propagação num volume de controle para um nível de tempo determinado.

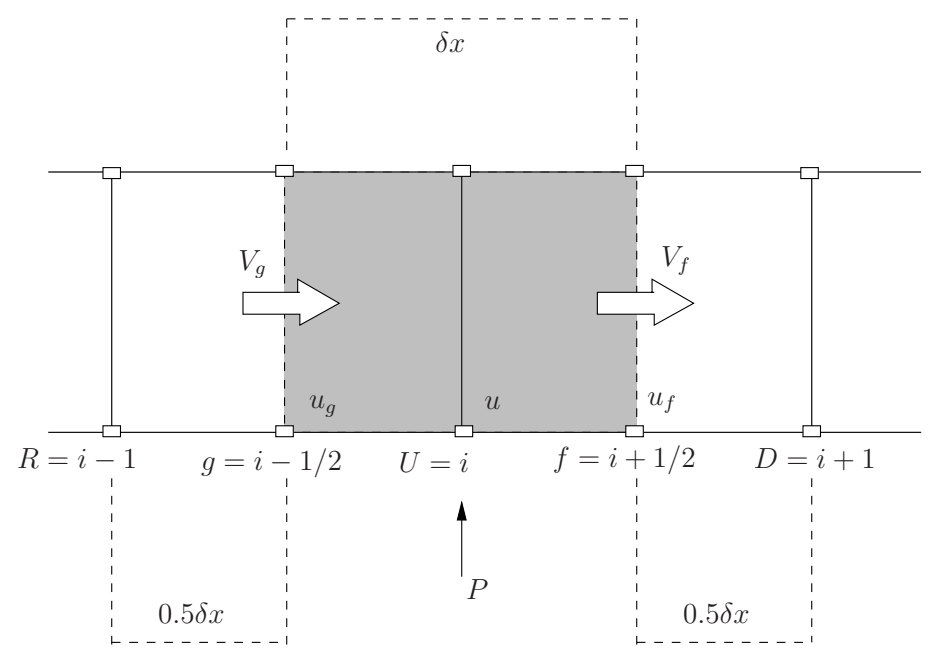

Figura 4.1: Representação esquemática em que se mostra as aproximações dos termos convectivos, mostrando as faces envolvidas $f$ e $g$ para a aproximação e a direção da velocidade $V_{f}$ $\left(V_{g}\right)$ de convecção na face $f(g)$.

\subsubsection{Equação de Burgers 1D}

A solução numérica da equação de Burgers (2.6) é calculada aproximando o termo difusivo pelo esquema de diferenças centrais de segundo ordem e o termo de advecção (na forma conservativa) é aproximada como

$$
\left.\frac{1}{2}\left(\frac{\partial u^{2}}{\partial x}\right)\right|_{i}=\frac{1}{2}\left(\frac{\bar{u}_{i+\frac{1}{2}} \cdot u_{i+\frac{1}{2}}-\bar{u}_{i-\frac{1}{2}} \cdot u_{i-\frac{1}{2}}}{2(\delta x / 2)}\right),
$$


em que as velocidades de advecção são aproximadas pelas médias

$$
\bar{u}_{i-\frac{1}{2}}=\frac{1}{2}\left(u_{i}+u_{i-1}\right) \quad \text { e } \quad \bar{u}_{i+\frac{1}{2}}=\frac{1}{2}\left(u_{i+1}+u_{i}\right)
$$

e a variavel convectada $u$ nas faces $i+1 / 2$ e $i-1 / 2$ do volume de controle são calculadas (por exemplo) pelos esquemas FDHERPUS, CSPUS e RUS. É importante observar que, com o uso da formula (4.4) para calcular as velocidades convectivas, a formula (4.3) fornece uma implementação simplificada dos esquemas upwind para a variável convectada $u$ com segunda ordem de precisão local (globalmente). No entanto, em regiões onde a soluçao é suficientemente suave a ordem de convergência do método global com estes esquemas pode ser melhorada pela implementação no contexto de reconstrução upwind das funções fluxo (ver, por exemplo, [84]). No código numérico próprio equipado com os esquemas upwind, tais como FDHERPUS, CSPUS e RUS, a condição de estabilidade para resolver a equação (2.6) e a escolha do número de Courant $C$ são feitas de maneira similar ao caso da equação de advecção 1D.

\subsubsection{Equações de Euler 1D}

O sistema hiperbólico (2.9) é numericamente resolvido pelo uso da fórmula conservativa de diferenças finitas explícitas

$$
\boldsymbol{U}_{i}^{n+1}=\boldsymbol{U}_{i}^{n}+\frac{\delta t}{\delta x}\left[\boldsymbol{F}_{i-1 / 2}^{n}-\boldsymbol{F}_{i+1 / 2}^{n}\right]
$$

onde o vetor fluxo na interface é avaliado pelo uso do método proposto por Roe e Pike (1984) [60], a saber: primeiro são calculados as médias de Roe (ver Toro (1999) [73]); depois, para $k=1,2,3$, os autovalores $\tilde{\lambda}$ e os autovetores $\tilde{K}^{(k)}$ da matriz jacobiana $\hat{A}$ (ambos avaliados utilizando médias) são determinados; a seguir são calculadas as onda (strengths) $\tilde{\alpha}$; e então com todas as quantidades acima mencionadas, os vetores fluxo $\boldsymbol{F}_{i-1 / 2}$ e $\boldsymbol{F}_{i+1 / 2}$ (omitindo os indices temporais por simplicidade) são diretamente aproximados pelo uso de limitadores de fluxo. Em particular, e de uma maneira similar, como foi feito por Hubbard e Garcia-Navarro (2000) [33], implementa-se o fluxo genérico $\boldsymbol{F}_{i+1 / 2}$ como

$$
\boldsymbol{F}_{i+1 / 2}=\boldsymbol{F}_{i+1 / 2}^{L O W}+\left.\frac{1}{2} \sum_{k=1}^{3} \operatorname{sign}\left(\tilde{\lambda}_{k}\right) \tilde{\lambda}_{k}\left(1-\left|\theta_{k}\right|\right) \varphi\left(r_{f}^{k}\right) \tilde{\alpha}_{k} \tilde{K}^{(k)}\right|_{i+1 / 2}
$$

onde $\boldsymbol{F}_{i+1 / 2}^{L O W}$ é um fluxo numérico de baixa ordem, $\theta_{k}=\tilde{\lambda}_{k} \delta t / \delta x$, e $\varphi(\cdot)$ é um limitador de fluxo tal como FDHERPUS, CSPUS ou RUS.

O sensor $r_{f}^{k}$ é calculado como

$$
r_{f}^{k}=\frac{\tilde{\alpha}_{k}^{u p w i n d}}{\tilde{\alpha}_{k}^{l o c a l}}
$$

onde $\tilde{\alpha}_{k}^{\text {local }}=\left.\tilde{\alpha}_{k}\right|_{i+1 / 2}$ e $\tilde{\alpha}_{k}^{\text {upwind }}$ é obtido a montante de acordo a velocidade $\tilde{\lambda}_{k}$ na face $i+1 / 2$. 
Finalmente, para o cálculo de $\boldsymbol{F}_{i+1 / 2}^{L O W}$ na equação (4.6) usa-se

$$
\boldsymbol{F}_{i+1 / 2}^{L O W}=\frac{1}{2}\left(\boldsymbol{F}_{i}+\boldsymbol{F}_{i+1}\right)-\frac{1}{2} \sum_{k=1}^{3} \tilde{\alpha}_{k}\left|\tilde{\lambda}_{k}\right| \tilde{K}^{(k)} .
$$

O método numérico para resolver as equações de Euler 1D é explícito, e sua estabilidade é governada pela condição CFL (ver Berthon (2005) [4]). Neste caso, o passo temporal $\delta t$ é assumido como

$$
\frac{\delta t}{\delta x} \max \left\{\left|\lambda_{i+1 / 2}^{-}\right|,\left|\lambda_{i+1 / 2}^{+}\right|\right\} \leq 1,
$$

onde $\lambda_{i+1 / 2}^{ \pm}$são as ondas numéricas (acústicas) associadas à função fluxo numérico.

\subsubsection{Equações de Euler 2D}

O sistema de equações hiperbólicos de Euler 2D (2.17) foi resolvido utilizando o software CLAWPACK de LeVeque (2006) [42], o qual é uma coleção de subrotinas em FORTRAN para resolução de sistemas de leis de conservação hiperbólicos unidimensionais e multidimensionais utilizando o método de volumes finitos sobre malhas retangulares uniformes. O software realiza cálculos multidimensionais sobre malhas cartesianas, geralmente sobre malhas retangulares. Além disso, o pacote inclui versões de refinamento de malha (AMRCLAW, ver LeVeque (1998) [38]) e uma versão de MPI (Message Passing Interface), em que o domínio pode ser distribuido entre múltiplos processadores. Em LeVeque (2004) [41] encontra-se uma descrição completa do algoritmo implementado no software, o qual é similar ao descrito para equações de Euler 1D. No código pode-se implementar os métodos de Godunov de primeira ordem de precisão ou uma variante de segunda ordem (método de primeira ordem com termo de correção), com os limitadores de fluxo dos esquemas FDHERPUS, RUS e CSPUS. Mais detalhes sobre o software CLAWPACK podem ser encontrados no apêndice A.

\subsubsection{Sistema de equações de águas rasas}

Para as discretizações dos sistemas de equações hiperbólicos de águas rasas 1D e 2D dados pelas equações (2.13) e (2.23) respectivamente, procede-se de maneira muito similar como foi feito na discretização das equações de Euler, motivo pelo qual será omitida sua descrição. O código computacional utilizado para resolver estes sistemas hiperbólicos não lineares foi, também, o CLAWPACK, equipado com os esquemas de alta resolução desenvolvidos neste trabalho.

\subsection{Equações de Navier Stokes}

Esta seção descreve a metodologia utilizada para solucionar numericamente as equações de Navier-Stokes (2.28). O ambiente de simulação genuinamente brasileiro Freeflow (ver por exemplo Castelo et al. (2000) [10]) desenvolvido no LMACC (Laboratório de Matemática Aplicada 
e Computação Científica) do ICMC-USP foi o código base para a simulação computacional de problemas 2D e 3D de escoamentos incompressíveis com superfícies livres móveis. Essa tecnologia numérica foi equipada com esquemas upwind e utilizada para simular fluxos incompressíveis. A estratégia numérica implementada no Freeflow é foi a GENSMAC (Generalized-SimpliedMarker -and-Cell), desenvolvida por Tomé e Mckee (2001) [71], uma variante do método de projeção de Chorin (1968) [11], que em linhas gerais tem como ideia central resolver explicitamente as equações de Navier-Stokes para escoamentos incompressíveis envolvendo superfícies livreis em domínios arbitrários sobre os quais especificam-se as condições de contorno apresentadas na seção 2.3 do capítulo 2. A metodologia numérica empregada é no contexto de diferenças finitas sobre malhas deslocadas (staggered grid) e utiliza partículas marcadoras virtuais para definir a posição da superfície livre do fluido, tais como os métodos MAC (1965) (Marker-and-Cell) de Harlow e Welch (1965) [29] e SMAC (1970) (Simplied-MAC) de Amsden e Harlow (1970) [2]. Nas malhas deslocadas, a pressão é avaliada no centro das células computacionais e as velocidades são calculadas nos centros das faces. A localização das variaveis dependentes nesse tipo de malha é ilustrada na figura 4.2 para o caso 3D. As derivadas temporais são aproximadas (por simplicidade) pelo método de Euler explícito enquanto que o gradiente de pressão e os termos convectivos por diferenças centrais de segunda ordem.

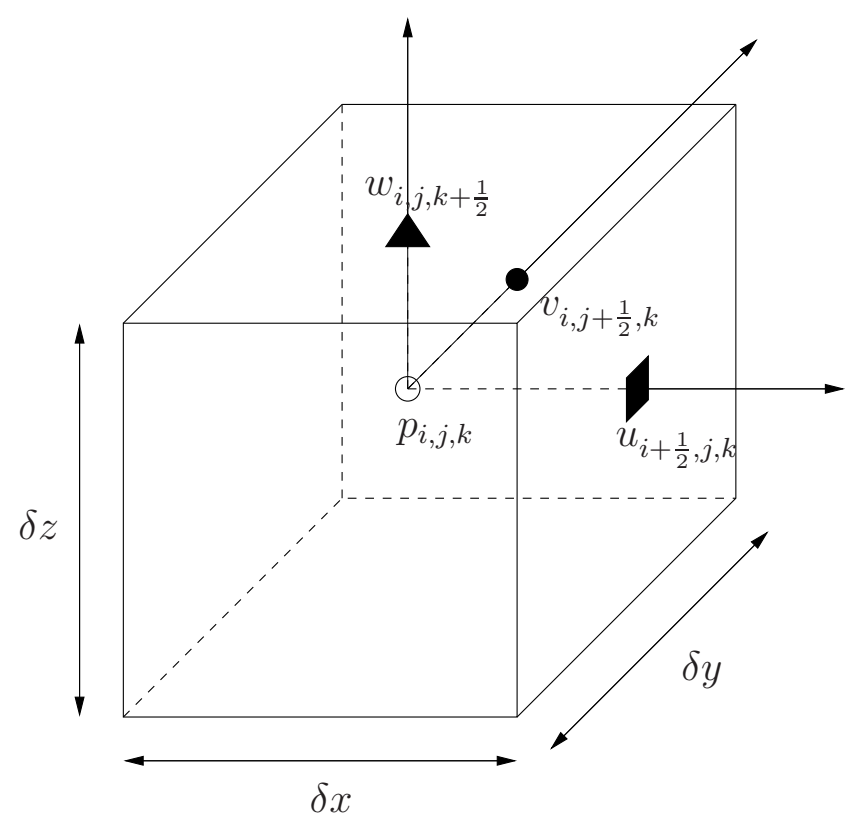

Figura 4.2: Célula computacional típica mostrando onde as variáveis dependentes são avaliadas.

\subsubsection{Aproximação dos termos convectivos e os esquemas upwind}

Os termos convectivos (não lineares) nas equações (2.28) são os principais causadores de dificuldades numéricas nas simulações e os responsáveis por fenômenos complexos no escoamento. Dessa forma, a aproximação desses termos merece atenção especial. Vale observar que a estabilidade, exatidão e limitação da solução numérica estão relacionados com o esquema adotado 
para aproximar os termos convectivos. Com o objetivo de mostrar como é feita a discretização dos termos convectivos, apresenta-se a seguir a aproximação desses termos no transporte do componente $u$ de velocidade (aproximações para os outros componentes seguem procedimentos semelhantes).

O termo não linear na primeira equação do sistema (2.28) é avaliado em $\left(i+\frac{1}{2}, j, k\right)$ e aproximado como

$$
\begin{aligned}
\left.\left(\frac{\partial(u u)}{\partial x}+\frac{\partial(u v)}{\partial y}+\frac{\partial(u w)}{\partial z}\right)\right|_{i+\frac{1}{2}, j, k} \approx & \frac{\bar{u}_{1} u_{i+1, j, k}-\bar{u}_{2} u_{i, j, k}}{\delta x}+\frac{\bar{v}_{1} u_{i+\frac{1}{2}, j+\frac{1}{2}, k}-\bar{v}_{2} u_{i+\frac{1}{2}, j-\frac{1}{2}, k}}{\delta y} \\
& +\frac{\bar{w}_{1} u_{i+\frac{1}{2}, j, k+\frac{1}{2}}-\bar{w}_{2} u_{i+\frac{1}{2}, j, k-\frac{1}{2}}}{\delta z}
\end{aligned}
$$

onde as velocidades convectivas $\bar{u}_{1}, \bar{u}_{2}, \bar{v}_{1}, \bar{v}_{2}, \bar{w}_{1}$ e $\bar{w}_{2}$ são aproximadas pelas médias simples. Para ilustração, considera-se a velocidade convectiva $\bar{v}_{1}$

$$
\bar{v}_{1}=\bar{v}_{i+\frac{1}{2}, j+\frac{1}{2}, k} \approx \frac{1}{2}\left(v_{i+1, j+\frac{1}{2}, k}+v_{i, j+\frac{1}{2}, k}\right) \quad \text { e } \quad \bar{v}_{2}=\bar{v}_{i+\frac{1}{2}, j-\frac{1}{2}, k} \approx \frac{1}{2}\left(v_{i+1, j-\frac{1}{2}, k}+v_{i, j-\frac{1}{2}, k}\right)
$$

É importante observar que os esquemas upwind em variáveis normalizadas, tal como FDHERPUS, RUS e CSPUS, são aplicados para aproximar os valores da variável $u$ (convectada) $u_{i+1, j, k}$, $u_{i, j, k}, u_{i+\frac{1}{2}, j+\frac{1}{2}, k}, u_{i+\frac{1}{2}, j-\frac{1}{2}, k}, u_{i+\frac{1}{2}, j, k+\frac{1}{2}}$ e $u_{i+\frac{1}{2}, j, k-\frac{1}{2}}$ que aparecem na equação (4.10). De fato, uma vez conhecidos os sentidos das velocidades convectivas $\bar{u}_{1}, \bar{u}_{2}, \bar{v}_{1}, \bar{v}_{2}, \bar{w}_{1}$ e $\bar{w}_{2}$ nas faces $i+1, i, j+\frac{1}{2}, j-\frac{1}{2}, k+\frac{1}{2}$ e $k-\frac{1}{2}$, respectivamente, as posições a jusante $D$, a montante $U$ e mais a montante $R$ ficam definidas automaticamente. Na sequência, apresentam-se essas aproximações upwind fazendo o uso do esquema FDHERPUS.

(1) Aproximações para $u_{i+1, j, k}$ :

- Quando $\bar{u}_{1}=u_{i+1, j, k}>0$ e $\hat{u}_{i+\frac{1}{2}, j, k}=\frac{u_{i+\frac{1}{2}, j, k}-u_{i-\frac{1}{2}, j, k}}{u_{i+\frac{3}{2}, j, k}-u_{i-\frac{1}{2}, j, k}} \quad$ tem-se que

$$
u_{i+1, j, k}=\left\{\begin{array}{lll}
u_{R}+\left(u_{D}-u_{R}\right)\left[-4 \hat{u}_{U}^{5}+10 \hat{u}_{U}^{4}-8 \hat{u}_{U}^{3}+\hat{u}_{U}^{2}+2 \hat{u}_{U}\right], & \text { se } & \hat{u}_{U} \in[0,1] \\
u_{U}, & \text { se } & \hat{u}_{U} \notin[0,1]
\end{array}\right.
$$

na qual

$$
D=\left(i+\frac{3}{2}, j, k\right), \quad U=\left(i+\frac{1}{2}, j, k\right), \quad R=\left(i-\frac{1}{2}, j, k\right)
$$


- Quando $\bar{u}_{1}=u_{i+1, j, k}<0$ e $\hat{u}_{i+\frac{3}{2}, j, k}=\frac{u_{i+\frac{3}{2}, j, k}-u_{i+\frac{5}{2}, j, k}}{u_{i+\frac{1}{2}, j, k}-u_{i+\frac{5}{2}, j, k}} \quad$ tem-se que

$$
u_{i+1, j, k}=\left\{\begin{array}{lll}
u_{R}+\left(u_{D}-u_{R}\right)\left[-4 \hat{u}_{U}^{5}+10 \hat{u}_{U}^{4}-8 \hat{u}_{U}^{3}+\hat{u}_{U}^{2}+2 \hat{u}_{U}\right], & \text { se } & \hat{u}_{U} \in[0,1], \\
u_{U}, & \text { se } & \hat{u}_{U} \notin[0,1]
\end{array}\right.
$$

na qual

$$
D=\left(i+\frac{1}{2}, j, k\right), \quad U=\left(i+\frac{3}{2}, j, k\right), \quad R=\left(i+\frac{5}{2}, j, k\right)
$$

(2) Aproximações para $u_{i, j, k}$ :

- Quando $\bar{u}_{2}=u_{i, j, k}>0$ e $\hat{u}_{i-\frac{1}{2}, j, k}=\frac{u_{i-\frac{1}{2}, j, k}-u_{i-\frac{3}{2}, j, k}}{u_{i+\frac{1}{2}, j, k}-u_{i-\frac{3}{2}, j, k}} \quad$ tem-se que

$$
u_{i, j, k}=\left\{\begin{array}{lll}
u_{R}+\left(u_{D}-u_{R}\right)\left[-4 \hat{u}_{U}^{5}+10 \hat{u}_{U}^{4}-8 \hat{u}_{U}^{3}+\hat{u}_{U}^{2}+2 \hat{u}_{U}\right], & \text { se } \hat{u}_{U} \in[0,1], \\
u_{U}, & \text { se } & \hat{u}_{U} \notin[0,1]
\end{array}\right.
$$

na qual

$$
D=\left(i+\frac{1}{2}, j, k\right), \quad U=\left(i-\frac{1}{2}, j, k\right), \quad R=\left(i-\frac{3}{2}, j, k\right)
$$

- Quando $\bar{u}_{2}=u_{i, j, k}<0$ e $\hat{u}_{i+\frac{1}{2}, j, k}=\frac{u_{i+\frac{1}{2}, j, k}-u_{i+\frac{3}{2}, j, k}}{u_{i-\frac{1}{2}, j, k}-u_{i+\frac{3}{2}, j, k}} \quad$ tem-se que

$$
u_{i+1, j, k}=\left\{\begin{array}{lll}
u_{R}+\left(u_{D}-u_{R}\right)\left[-4 \hat{u}_{U}^{5}+10 \hat{u}_{U}^{4}-8 \hat{u}_{U}^{3}+\hat{u}_{U}^{2}+2 \hat{u}_{U}\right], & \text { se } & \hat{u}_{U} \in[0,1], \\
u_{U}, & \text { se } & \hat{u}_{U} \notin[0,1]
\end{array}\right.
$$

na qual

$$
D=\left(i-\frac{1}{2}, j, k\right), \quad U=\left(i+\frac{1}{2}, j, k\right), \quad R=\left(i+\frac{3}{2}, j, k\right) ;
$$

(3) Aproximações para $u_{i+\frac{1}{2}, j+\frac{1}{2}, k}$ :

- Quando $\bar{v}_{1}=v_{i+\frac{1}{2}, j+\frac{1}{2}, k}>0$ e $\hat{u}_{i+\frac{1}{2}, j, k}=\frac{u_{i+\frac{1}{2}, j, k}-u_{i+\frac{1}{2}, j-1, k}}{u_{i+\frac{1}{2}, j+1, k}-u_{i+\frac{1}{2}, j-1, k}}$ tem-se que

$$
u_{i+\frac{1}{2}, j+\frac{1}{2}, k}=\left\{\begin{array}{lll}
u_{R}+\left(u_{D}-u_{R}\right)\left[-4 \hat{u}_{U}^{5}+10 \hat{u}_{U}^{4}-8 \hat{u}_{U}^{3}+\hat{u}_{U}^{2}+2 \hat{u}_{U}\right], & \text { se } \hat{u}_{U} \in[0,1], \\
u_{U}, & \text { se } \hat{u}_{U} \notin[0,1]
\end{array}\right.
$$

na qual 


$$
D=\left(i+\frac{1}{2}, j+1, k\right), \quad U=\left(i+\frac{1}{2}, j, k\right), \quad R=\left(i+\frac{1}{2}, j-1, k\right) ;
$$

- Quando $\bar{v}_{1}=v_{i+\frac{1}{2}, j+\frac{1}{2}, k}<0$ e $\hat{u}_{i+\frac{1}{2}, j+1, k}=\frac{u_{i+\frac{1}{2}, j+1, k}-u_{i+\frac{1}{2}, j+2, k}}{u_{i+\frac{1}{2}, j, k}-u_{i+\frac{1}{2}, j+2, k}}$ tem-se que

$$
u_{i+\frac{1}{2}, j+\frac{1}{2}, k}=\left\{\begin{array}{lll}
u_{R}+\left(u_{D}-u_{R}\right)\left[-4 \hat{u}_{U}^{5}+10 \hat{u}_{U}^{4}-8 \hat{u}_{U}^{3}+\hat{u}_{U}^{2}+2 \hat{u}_{U}\right], & \text { se } & \hat{u}_{U} \in[0,1] \\
u_{U}, & \text { se } & \hat{u}_{U} \notin[0,1]
\end{array}\right.
$$

na qual

$$
D=\left(i+\frac{1}{2}, j, k\right), \quad U=\left(i+\frac{1}{2}, j+1, k\right), \quad R=\left(i+\frac{1}{2}, j+2, k\right) ;
$$

(4) Aproximações para $u_{i+\frac{1}{2}, j-\frac{1}{2}, k}$ :

- Quando $\bar{v}_{2}=v_{i+\frac{1}{2}, j-\frac{1}{2}, k}>0$ e $\hat{u}_{i+\frac{1}{2}, j-1, k}=\frac{u_{i+\frac{1}{2}, j-1, k}-u_{i+\frac{1}{2}, j-2, k}}{u_{i+\frac{1}{2}, j, k}-u_{i+\frac{1}{2}, j-2, k}}$ tem-se que

$$
u_{i+\frac{1}{2}, j-\frac{1}{2}, k}=\left\{\begin{array}{lll}
u_{R}+\left(u_{D}-u_{R}\right)\left[-4 \hat{u}_{U}^{5}+10 \hat{u}_{U}^{4}-8 \hat{u}_{U}^{3}+\hat{u}_{U}^{2}+2 \hat{u}_{U}\right], & \text { se } & \hat{u}_{U} \in[0,1], \\
u_{U}, & \text { se } & \hat{u}_{U} \notin[0,1]
\end{array}\right.
$$

na qual

$$
D=\left(i+\frac{1}{2}, j, k\right), \quad U=\left(i+\frac{1}{2}, j-1, k\right), \quad R=\left(i+\frac{1}{2}, j-2, k\right) ;
$$

- Quando $\bar{v}_{2}=v_{i+\frac{1}{2}, j-\frac{1}{2}, k}<0$ e $\hat{u}_{i+\frac{1}{2}, j, k}=\frac{u_{i+\frac{1}{2}, j, k}-u_{i+\frac{1}{2}, j+1, k}}{u_{i+\frac{1}{2}, j-1, k}-u_{i+\frac{1}{2}, j+1, k}}$ tem-se que

$$
u_{i+\frac{1}{2}, j-\frac{1}{2}, k}=\left\{\begin{array}{lll}
u_{R}+\left(u_{D}-u_{R}\right)\left[-4 \hat{u}_{U}^{5}+10 \hat{u}_{U}^{4}-8 \hat{u}_{U}^{3}+\hat{u}_{U}^{2}+2 \hat{u}_{U}\right], & \text { se } \hat{u}_{U} \in[0,1], \\
u_{U}, & \text { se } \hat{u}_{U} \notin[0,1],
\end{array}\right.
$$

na qual

$$
D=\left(i+\frac{1}{2}, j-1, k\right), \quad U=\left(i+\frac{1}{2}, j, k\right), \quad R=\left(i+\frac{1}{2}, j+1, k\right) ;
$$

(5) Aproximações para $u_{i+\frac{1}{2}, j, k+\frac{1}{2}}$ :

- Quando $\bar{w}_{1}=w_{i+\frac{1}{2}, j, k+\frac{1}{2}}>0$ e $\hat{u}_{i+\frac{1}{2}, j, k}=\frac{u_{i+\frac{1}{2}, j, k}-u_{i+\frac{1}{2}, j, k-1}}{u_{i+\frac{1}{2}, j, k+1}-u_{i+\frac{1}{2}, j, k-1}}$ tem-se que

$$
u_{i+\frac{1}{2}, j, k+\frac{1}{2}}=\left\{\begin{array}{lll}
u_{R}+\left(u_{D}-u_{R}\right)\left[-4 \hat{u}_{U}^{5}+10 \hat{u}_{U}^{4}-8 \hat{u}_{U}^{3}+\hat{u}_{U}^{2}+2 \hat{u}_{U}\right], & \text { se } \hat{u}_{U} \in[0,1], \\
u_{U}, & \text { se } \hat{u}_{U} \notin[0,1]
\end{array}\right.
$$

na qual 


$$
D=\left(i+\frac{1}{2}, j, k+1\right), \quad U=\left(i+\frac{1}{2}, j, k\right), \quad R=\left(i+\frac{1}{2}, j, k-1\right)
$$

- Quando $\bar{w}_{1}=w_{i+\frac{1}{2}, j, k+\frac{1}{2}}<0$ e $\hat{u}_{i+\frac{1}{2}, j, k+1}=\frac{u_{i+\frac{1}{2}, j, k+1}-u_{i+\frac{1}{2}, j, k+2}}{u_{i+\frac{1}{2}, j, k}-u_{i+\frac{1}{2}, j, k+2}}$ tem-se que

$$
u_{i+\frac{1}{2}, j, k+\frac{1}{2}}=\left\{\begin{array}{lll}
u_{R}+\left(u_{D}-u_{R}\right)\left[-4 \hat{u}_{U}^{5}+10 \hat{u}_{U}^{4}-8 \hat{u}_{U}^{3}+\hat{u}_{U}^{2}+2 \hat{u}_{U}\right], & \text { se } \hat{u}_{U} \in[0,1], \\
u_{U}, & \text { se } \hat{u}_{U} \notin[0,1]
\end{array}\right.
$$

na qual

$$
D=\left(i+\frac{1}{2}, j, k\right), \quad U=\left(i+\frac{1}{2}, j, k+1\right), \quad R=\left(i+\frac{1}{2}, j, k+2\right)
$$

(6) Aproximações para $u_{i+\frac{1}{2}, j, k-\frac{1}{2}}$ :

- Quando $\bar{w}_{2}=w_{i+\frac{1}{2}, j, k-\frac{1}{2}}>0$ e $\hat{u}_{i+\frac{1}{2}, j, k-1}=\frac{u_{i+\frac{1}{2}, j, k-1}-u_{i+\frac{1}{2}, j, k-2}}{u_{i+\frac{1}{2}, j, k}-u_{i+\frac{1}{2}, j, k-2}}$ tem-se que

$$
u_{i+\frac{1}{2}, j, k-\frac{1}{2}}=\left\{\begin{array}{lll}
u_{R}+\left(u_{D}-u_{R}\right)\left[-4 \hat{u}_{U}^{5}+10 \hat{u}_{U}^{4}-8 \hat{u}_{U}^{3}+\hat{u}_{U}^{2}+2 \hat{u}_{U}\right], & \text { se } \hat{u}_{U} \in[0,1], \\
u_{U}, & \text { se } \hat{u}_{U} \notin[0,1]
\end{array}\right.
$$

na qual

$$
D=\left(i+\frac{1}{2}, j, k\right), \quad U=\left(i+\frac{1}{2}, j, k-1\right), \quad R=\left(i+\frac{1}{2}, j, k-2\right)
$$

- Quando $\bar{w}_{2}=w_{i+\frac{1}{2}, j, k-\frac{1}{2}}<0$ e $\hat{u}_{i+\frac{1}{2}, j, k}=\frac{u_{i+\frac{1}{2}, j, k}-u_{i+\frac{1}{2}, j, k+1}}{u_{i+\frac{1}{2}, j, k-1}-u_{i+\frac{1}{2}, j, k+1}}$ tem-se que

$$
u_{i+\frac{1}{2}, j, k-\frac{1}{2}}=\left\{\begin{array}{lll}
u_{R}+\left(u_{D}-u_{R}\right)\left[-4 \hat{u}_{U}^{5}+10 \hat{u}_{U}^{4}-8 \hat{u}_{U}^{3}+\hat{u}_{U}^{2}+2 \hat{u}_{U}\right], & \text { se } \hat{u}_{U} \in[0,1], \\
u_{U}, & \text { se } \hat{u}_{U} \notin[0,1]
\end{array}\right.
$$

na qual

$$
D=\left(i+\frac{1}{2}, j, k-1\right), \quad U=\left(i+\frac{1}{2}, j, k\right), \quad R=\left(i+\frac{1}{2}, j, k+1\right)
$$

\subsubsection{Algoritmo computacional}

Será apresentado agora um ciclo computacional para simular escoamentos incompressíveis com superfícies livres móveis governados pelas equações de Navier-Stokes. O ciclo consiste em atualizar as variáveis dependentes (discretas) a partir do conhecimento delas num tempo inicial $t_{0}$. Desse modo o campo de velocidade no tempo $t_{n+1}=t_{n}+\delta t$ é calculado através dos seguintes passos (maiores detalhes podem ser encontrados em Ferreira (2001) [19] - Capítulo 3): 
-Passo 1: Considere um campo de pressão tentativo $\tilde{p}=p^{n}$ que satisfaz as condições de contorno (2.42) na superfície livre do fluido e as equações (2.43) e (2.44) para o campo de velocidade nesta fronteira.

-Passo 2: Calcula-se um campo de velocidade intermediário $\tilde{u}_{i}$ usando-se a aproximação explícita

$$
\tilde{u}_{i}=u_{i}+\delta t\left[-\frac{\partial\left(u_{i} u_{j}\right)}{\partial x_{j}}-\frac{\partial \tilde{p}}{\partial x_{i}}+\frac{1}{R e} \frac{\partial}{\partial x_{j}}\left(\frac{\partial u_{i}}{\partial x_{j}}\right)+\frac{1}{F r^{2}} g_{i}\right], \quad i=1,2,3,
$$

-Passo 3: Resolve-se a equação de Poisson para o potencial auxiliar $\zeta$

$$
\frac{\partial}{\partial x_{i}}\left(\frac{\partial \zeta}{\partial x_{i}}\right)=\left(\frac{\partial \tilde{u}_{i}}{\partial x_{i}}\right), \quad i=1,2,3,
$$

com condição de Dirichlet homogênea na superfície livre e na saída do domínio computacional, e com condição homogênea de Neumann na região de entrada e no contorno rígido. A equação de Poisson discretizada gera um sistema linear que é resolvido por meio do método dos gradientes conjugados (ver Hestenes and Stiefel (1952) [32] ou Trefethen and Bau (1997) [74]).

-Passo 4: Atualiza-se o campo de velocidade por meio de

$$
u_{i}=\tilde{u}_{i}-\frac{\partial \zeta}{\partial x_{i}}, \quad i=1,2,3 .
$$

-Passo 5: Atualiza-se o campo de pressão por (ver Tomé e McKee [72])

$$
p=\tilde{p}+\frac{\zeta}{\delta t} .
$$

-Passo 6: Atualizam-se as posições das partículas marcadoras que representam o fluido. Estas partículas permitem a visualização do escoamento e a orientação da superfície livre. Essas posições são determinadas resolvendo-se a seguinte equação diferencial

$$
\frac{d x_{i}}{d t}=u_{i}, \quad i=1,2,3,
$$

pelo método de Euler explícito. Retorna-se ao Passo 1 para dar início ao próximo ciclo de cálculo. 


\section{Derivação dos esquemas convectivos}

Neste capítulo são derivados os esquemas upwind de alta resolução propostos neste trabalho, a saber FDHERPUS , CSPUS, RUS e uma modificação do esquema RUS (MRUS) na qual introduz-se uma constante $\varepsilon$ na função limitadora com a finalidade de evitar oscilações não físicas (e, consequentemente, instabilidades numéricas) nas soluções computadas.

\subsection{O esquema FDHERPUS}

Para a derivação do esquema upwind FDHERPUS, foi utilizada a técnica de interpolação de Hermite que tem como objetivo representar uma função $g$ por um polinómio que seja o interpolador de $g$ sobre alguns pontos de seu domínio; também exige-se que a derivada seja o interpolador da derivada de $g$ sobre esses mesmos pontos (detalhes podem ser encontrados em Burdem (2010) [8]). Admitindo que $g \in C^{1}([0,1])$ e que $\hat{\phi}_{U i}, i=0,1,2$ são pontos distintos em $[0,1]$, então existe um único polinómio $\hat{\phi}_{f} \in P_{5}\left[\hat{\phi}_{U}\right]$ (ver [8]) que verifica

$$
\hat{\phi}_{f}\left(\hat{\phi}_{U i}\right)=g\left(\hat{\phi}_{U i}\right), \quad \hat{\phi}_{f}^{\prime}\left(\hat{\phi}_{U i}\right)=g^{\prime}\left(\hat{\phi}_{U i}\right), \quad i=0,1,2 .
$$

O polinómio $\hat{\phi}_{f}$ é definido por

$$
\hat{\phi}_{f}=\sum_{k=0}^{2}\left[h_{k}\left(\hat{\phi}_{U}\right) g\left(\hat{\phi}_{U k}\right)+v_{k}\left(\hat{\phi}_{U}\right) g^{\prime}\left(\hat{\phi}_{U k}\right)\right], \quad \text { se } \quad \hat{\phi}_{U} \in[0,1] \text {, }
$$

em que $h_{k}\left(\hat{\phi}_{U}\right)$ e $v_{k}\left(\hat{\phi}_{U}\right)$ são definidos respectivamente como

$$
h_{k}\left(\hat{\phi}_{U}\right)=\left[l_{k}\left(\hat{\phi}_{U}\right)\right]^{2}\left(1-2 l_{k}^{\prime}\left(\hat{\phi}_{U k}\right)\left(\hat{\phi}_{U}-\hat{\phi}_{U k}\right)\right)
$$




$$
v_{k}\left(\hat{\phi}_{U}\right)=\left[l_{k}\left(\hat{\phi}_{U}\right)\right]^{2}\left(\hat{\phi}_{U}-\hat{\phi}_{U k}\right)
$$

e $l_{k}$ como

$$
l_{k}\left(\hat{\phi}_{U}\right)=\prod_{i=0, i \neq k}^{2} \frac{\hat{\phi}_{U}-\hat{\phi}_{U i}}{\hat{\phi}_{U k}-\hat{\phi}_{U i}}, \quad \text { (forma de Lagrange). }
$$

Em resumo, $\hat{\phi}_{f}$ é o polinómio interpolador de Hermite (de quinto grau) que será utilizado para interpolar $g$ sobre os pontos $O, Q$ e $P$ mencionados no capítulo 3.

Para determinar o esquema FDHERPUS, consideram-se as condições de Leonard (1988) [37], isto é

- passar por $O(0,0)$ e $P(1,1)$, isto é, $\hat{\phi}_{f}(0)=0$ e $\hat{\phi}_{f}(1)=1$,

- passar por $Q\left(\frac{1}{2}, \frac{3}{4}\right)$, ou seja, $\hat{\phi}_{f}\left(\frac{1}{2}\right)=\frac{3}{4}$,

- passar por $Q$ com inclinação $\frac{3}{4}$ ou, equivalentemente, $\hat{\phi}_{f}^{\prime}\left(\frac{1}{2}\right)=\frac{3}{4}$,

mais as duas condições de diferenciabilidade

- $\hat{\phi}_{f}^{\prime}(0)=2$,

- $\hat{\phi}_{f}^{\prime}(1)=0$.

Levando essas condições na equação (5.2) resulta

$$
\hat{\phi}_{f}=2 v_{0}\left(\hat{\phi}_{U}\right)+\frac{3}{4} h_{1}\left(\hat{\phi}_{U}\right)+\frac{3}{4} v_{1}\left(\hat{\phi}_{U}\right)+h_{2}\left(\hat{\phi}_{U}\right) .
$$

Para determinar expressões para $v_{k}$ e $h_{k}$, aplicam-se as equações (5.3), (5.4) e (5.5) na equação (5.6) para obter

$$
\begin{aligned}
& v_{0}\left(\hat{\phi}_{U}\right)=4 \hat{\phi}_{U}^{5}-12 \hat{\phi}_{U}^{4}+13 \hat{\phi}_{U}^{3}-6 \hat{\phi}_{U}^{2}+\hat{\phi}_{U}, \\
& h_{1}\left(\hat{\phi}_{U}\right)=16 \hat{\phi}_{U}^{4}-32 \hat{\phi}_{U}^{3}+16 \hat{\phi}_{U}^{2}, \\
& v_{1}\left(\hat{\phi}_{U}\right)=16 \hat{\phi}_{U}^{5}-40 \hat{\phi}_{U}^{4}+32 \hat{\phi}_{U}^{3}-8 \hat{\phi}_{U}^{2}, \\
& h_{2}\left(\hat{\phi}_{U}\right)=-24 \hat{\phi}_{U}^{5}-52 \hat{\phi}_{U}^{4}-34 \hat{\phi}_{U}^{3}+7 \hat{\phi}_{U}^{2} .
\end{aligned}
$$

Substituindo na equação (5.6) as equações (5.7)-(5.10) obtém-se o esquema FDHERPUS em variáveis normalizadas, que em resumo é dado por 


$$
\hat{\phi}_{f}=\left\{\begin{array}{lll}
-4 \hat{\phi}_{U}^{5}+10 \hat{\phi}_{U}^{4}-8 \hat{\phi}_{U}^{3}+\hat{\phi}_{U}^{2}+2 \hat{\phi}_{U}, & \text { se } & \hat{\phi}_{U} \in[0,1] \\
\hat{\phi}_{U}, & \text { se } & \hat{\phi}_{U} \notin[0,1] .
\end{array}\right.
$$

A segunda sentença na equação (5.11) é imposta levando em conta a sugestão de Leonard (1988) [37], isto é para $\hat{\phi}_{U} \notin[0,1]$ considera-se o esquema FOU de Courant (1952) [14]. O esquema FDHERPUS em variáveis não normalizadas (e que está implemetado nos programas computacionais) é então dado por

$$
\phi_{f}=\left\{\begin{array}{lll}
\phi_{R}+\left(\phi_{D}-\phi_{R}\right)\left(-4 \hat{\phi}_{U}^{5}+10 \hat{\phi}_{U}^{4}-8 \hat{\phi}_{U}^{3}+\hat{\phi}_{U}^{2}+2 \hat{\phi}_{U}\right), & \text { se } \hat{\phi}_{U} \in[0,1], \\
\phi_{U}, & \text { se } \quad \hat{\phi}_{U} \notin[0,1] .
\end{array}\right.
$$

O limitador de fluxo correspondente $\varphi$ para o esquema FDHERPUS é deduzido usando-se a metodologia de combinar um método de primeira ordem com um de ordem superior por meio de uma função não-linear (ver por exemplo Waterson e Deconinck (2007) [81]). O método consiste em substituir, para $\hat{\phi}_{U} \in[0,1]$, as equações $(5.11)$ e $\hat{\phi}_{U}=\frac{r_{f}}{1+r_{f}}$ na combinção

$$
\hat{\phi}_{f}=\hat{\phi}_{U}+\frac{1}{2} \varphi\left(r_{f}\right)\left(1-\hat{\phi}_{U}\right)
$$

obtém-se

$$
-4\left(\frac{r_{f}}{1+r_{f}}\right)^{5}+10\left(\frac{r_{f}}{1+r_{f}}\right)^{4}-8\left(\frac{r_{f}}{1+r_{f}}\right)^{3}+\left(\frac{r_{f}}{1+r_{f}}\right)^{2}+\frac{r_{f}}{1+r_{f}}=\frac{1}{2} \varphi\left(r_{f}\right)\left(\frac{1}{1+r_{f}}\right)
$$

ou (isolando o limitador)

$$
\varphi\left(r_{f}\right)=\frac{2 r_{f}^{4}+2 r_{f}^{3}+10 r_{f}^{2}+2 r_{f}}{\left(1+r_{f}\right)^{4}}
$$

Para $\hat{\phi}_{U} \notin[0,1]$, considera-se $\hat{\phi}_{f}=\hat{\phi}_{U}$, que ao substituir na equação (5.13) resulta em $\varphi\left(r_{f}\right)=0$. Pode-se notar que devido à $\hat{\phi}_{U} \in[0,1], r_{f} \geq 0$. De fato

$$
0 \leq \hat{\phi}_{U} \leq 1 \quad \Leftrightarrow \quad 0 \leq \frac{r_{f}}{1+r_{f}} \leq 1
$$

a partir da qual, resolvendo-se as inequações, obtém-se $r_{f} \geq 0$.

Em resumo, o limitador FDHERPUS fica

$$
\varphi\left(r_{f}\right)= \begin{cases}\frac{2 r_{f}^{4}+2 r_{f}^{3}+10 r_{f}^{2}+2 r_{f}}{\left(1+r_{f}\right)^{4}}, & \text { se } \quad r_{f} \geq 0 \\ 0, & \text { se } \quad r_{f}<0\end{cases}
$$

e satisfaz a propriedade (3.17) para segunda ordem de precisão ([81]), isto é 


$$
\varphi(1)=\frac{2(1)^{4}+2(1)^{3}+10(1)^{2}+2(1)}{2^{4}}=1 .
$$

Além disso o limitador FDHERPUS satisfaz a propiedade (3.18) (Zijlema (1996) [85]) para atingir terceira ordem de precisão, isto é

$$
\begin{gathered}
\varphi^{\prime}\left(r_{f}\right)=\frac{\left[8 r_{f}^{3}+6 r_{f}^{2}+20 r_{f}+2\right]\left(1+r_{f}\right)^{4}-\left[2 r_{f}^{4}+2 r_{f}^{3}+10 r_{f}^{2}+2 r_{f}\right]\left(1+r_{f}\right)^{4}}{\left(1+r_{f}\right)^{8}}, \\
\varphi^{\prime}(1)=\frac{\left[8(1)^{3}+6(1)^{2}+20(1)+2\right](1+1)^{4}-\left[2(1)^{4}+2(1)^{3}+10(1)^{2}+2\right](1+1)^{4}}{(1+1)^{8}}=\frac{1}{4} .
\end{gathered}
$$

Ainda mais, o limitador FDHERPUS verifica o princípio de monoticidade de Sweby (1984) [67], isto é,

$$
\varphi^{\prime}\left(r_{f}\right) \longrightarrow 2 \text { quando } \quad r_{f} \longrightarrow 0 \text {. }
$$

As figuras 5.1 e 5.2 apresentam o esquema FDHERPUS e o seu limitador de fluxo correspondente nos planos $\hat{\phi}_{U} \perp \hat{\phi}_{f}$ e $r_{f} \perp \varphi\left(r_{f}\right)$ respectivamente, onde pode-se observar que o gráfico fica dentro das regiões TVD.

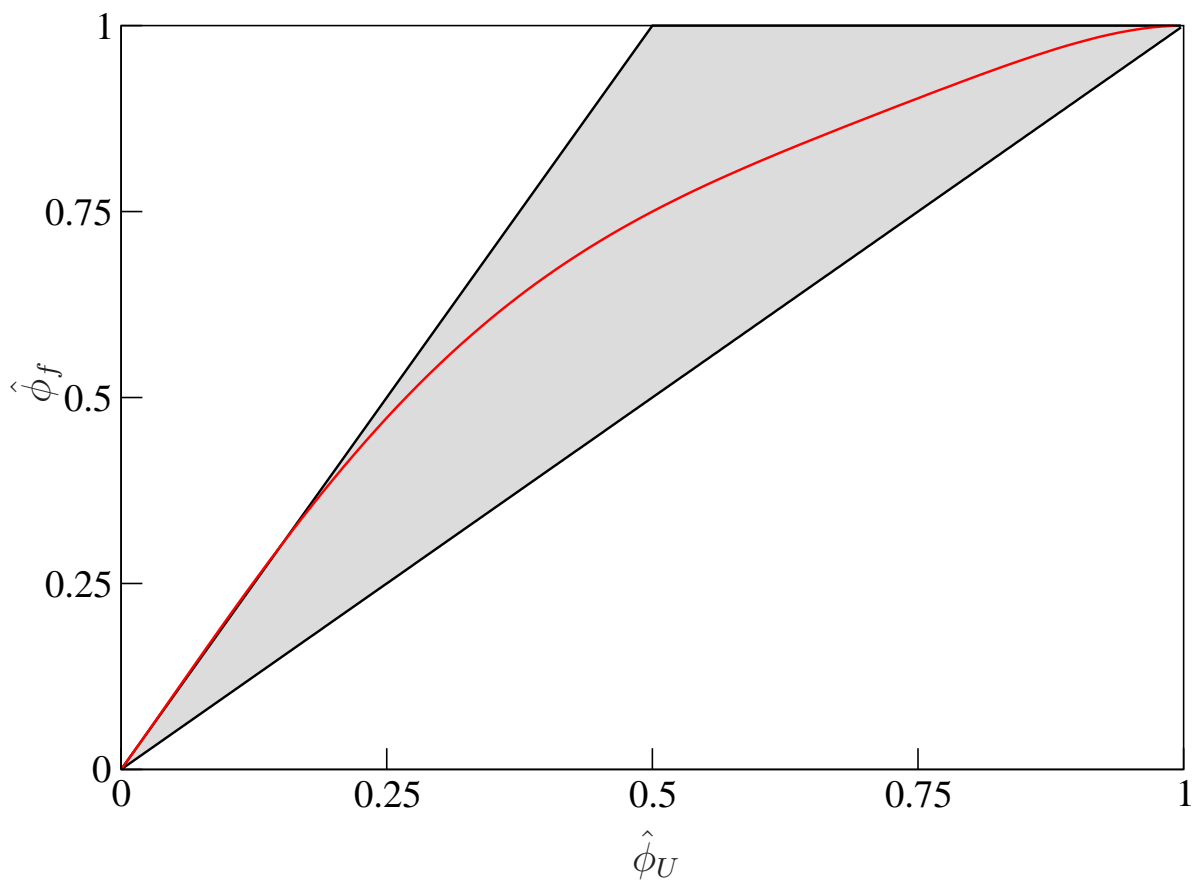

Figura 5.1: Esquema FDHERPUS na região TVD em variáveis normalizadas. 


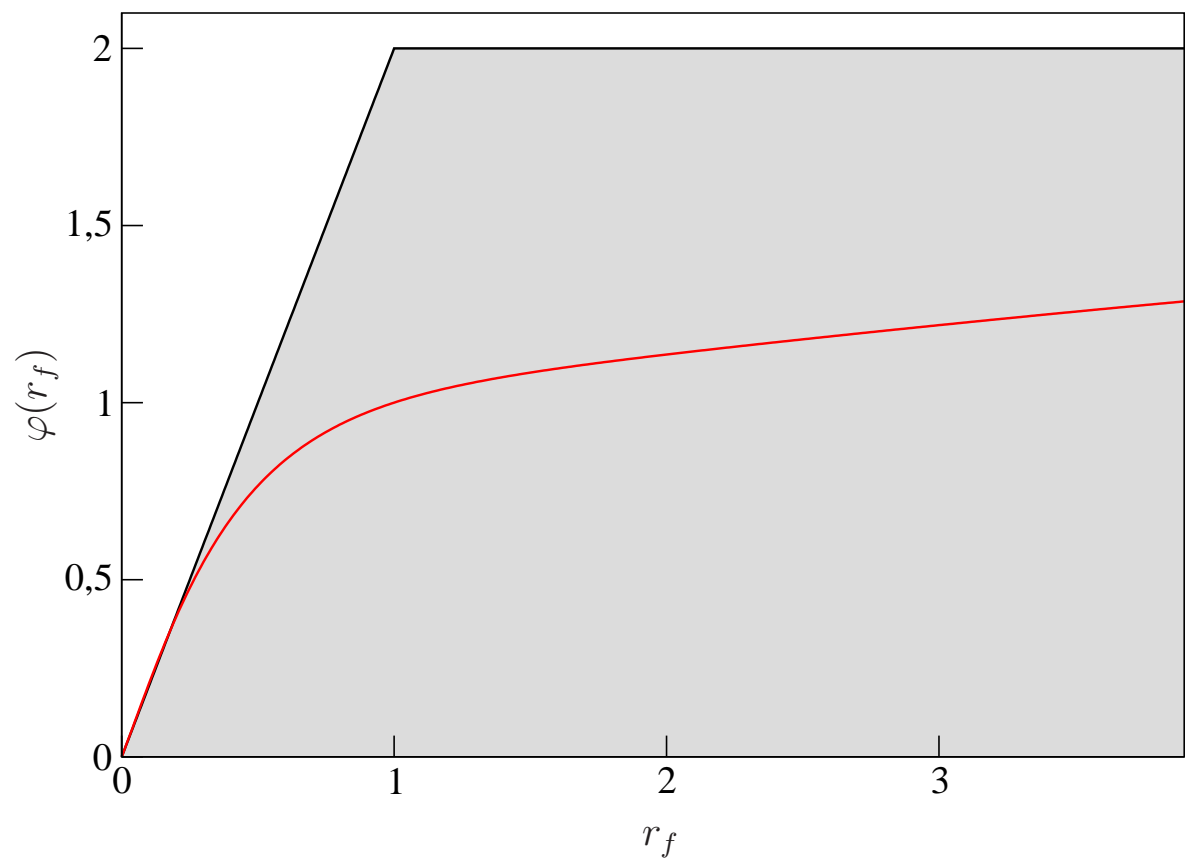

Figura 5.2: Limitador de fluxo para o esquema FDHERPUS na região TVD.

\subsection{O esquema CSPUS}

Para derivar o esquema CSPUS, considera-se a interpolação por splines cúbica (para detalhes ver Burden (2010) [8]). A ideia básica é definir uma partição $\mathscr{P}=\left\{0=x_{0}<x<x_{2}=1\right\}$ do intervalo $[0,1]$, com $x_{1}=1 / 2$, considerar os valores a interpolar $g\left(x_{0}\right)=0, g\left(x_{1}\right)=3 / 4 \mathrm{e}$ $g\left(x_{2}\right)=1$ (valores estes tomados segundo as condições de Leonard (1988) [37] apresentadas na subseção 3.2) e, então, construir funções polinomiais (do terceiro grau) em cada subintervalo $I_{i}=\left[x_{i}, x_{i+1}\right], i=0,1$ (por partes) que interpolam os valores conhecidos $g\left(x_{i}\right), i=0,1,2$. Matematicamente, o processo consiste em encontrar

$$
S_{h} \in V_{h}=\left\{q_{h} \in C^{2}([0,1]) ;\left.q_{h}\right|_{I_{i}} \in P_{3}\left[\hat{\phi}_{U}\right], i=0,1\right\},
$$

tais que

(a) $\left.S_{h}\right|_{I_{i}}\left(x_{i}\right)=g\left(x_{i}\right), \quad$ e $\left.\quad S_{h}\right|_{I_{i}}\left(x_{i+1}\right)=g\left(x_{i+1}\right), \quad i=0,1$;

(b) $\left.S_{h}\right|_{I_{i+1}}\left(x_{i+1}\right)=\left.S_{h}\right|_{I_{i}}\left(x_{i+1}\right), \quad i=0$;

(c) $\left.S_{h}^{\prime}\right|_{I_{i+1}}\left(x_{i+1}\right)=\left.S_{h}^{\prime}\right|_{I_{i}}\left(x_{i+1}\right), \quad i=0$;

(d) $\left.S_{h}^{\prime \prime}\right|_{I_{i+1}}\left(x_{i+1}\right)=\left.S_{h}^{\prime \prime}\right|_{I_{i}}\left(x_{i+1}\right), \quad i=0$;

(e) $S_{h}^{\prime}\left(x_{0}\right)=g^{\prime}\left(x_{0}\right)$ e $S_{h}^{\prime}\left(x_{2}\right)=g^{\prime}\left(x_{2}\right)$ (condições adicionais).

Um polinônio cúbico envolve quatro constantes, fornecendo flexibilidade suficiente no procedimento de interpolação; isso assegura que a função interpoladora global não seja só derivavel no 
intervalo, mas também tenha segunda derivada continua. O esquema CSPUS é construído com base em splines cúbicas sobre dois intervalos $I_{0}=\left[x_{0}, x_{1}\right]=[0,1 / 2]$ e $I_{1}=\left[x_{1}, x_{2}\right]=[1 / 2,1]$, como definido em (5.21), da forma

$$
S_{h}(x)= \begin{cases}\left.S_{h}\right|_{I_{0}}(x), & \text { se } x \in I_{0}=\left[0, \frac{1}{2}\right], \\ \left.S_{h}\right|_{I_{1}}(x), & \text { se } x \in I_{1}=\left[\frac{1}{2}, 1\right]\end{cases}
$$

em que

$$
\left.S_{h}\right|_{I_{i}}(x)=a_{i}+b_{i}\left(x-x_{i}\right)+c_{i}\left(x-x_{i}\right)^{2}+d_{i}\left(x-x_{i}\right)^{3}, \quad i=0,1,
$$

sendo $a_{i}, b_{i}, c_{i}$ e $d_{i}, i=0,1$ coeficientes a serem determinados. Como resultado da aplicação da condição $(a)$ em (5.23) obtém-se

$$
\left.S_{h}\right|_{I_{i}}\left(x_{i}\right)=a_{i}=g\left(x_{i}\right), \quad i=0,1,2
$$

onde $a_{2}=g\left(x_{2}\right)$ (constante auxiliar). Da aplicação da condição (b) em (5.23) e definindo $h_{i}=x_{i+1}-x_{i}$, tem-se

$$
a_{i+1}=a_{i}+b_{i} h_{i}+c_{i} h_{i}^{2}+d_{i} h_{i}^{3}, \quad i=0,1
$$

Derivando (5.23) obtém-se

$$
\left.S_{h}^{\prime}\right|_{I_{i}}(x)=b_{i}+2 c_{i}\left(x-x_{i}\right)+3 d_{i}\left(x-x_{i}\right)^{2},
$$

o que implica que $\left.S_{h}^{\prime}\right|_{I_{i}}\left(x_{i}\right)=b_{i}$ para cada $i=0,1$. Definindo a constante auxiliar $b_{2}=S_{h}^{\prime}\left(x_{2}\right)$ e aplicando a condição $(c)$, tem-se

$$
b_{i+1}=b_{i}+2 c_{i} h_{i}+3 d_{i} h_{i}^{2}, \quad i=0,1 .
$$

Obtém-se outra relação entre os coeficientes de $\left.S_{h}\right|_{I_{i}}$ definindo a constante auxiliar $c_{2}=$ $S_{h}^{\prime \prime}\left(x_{2}\right) / 2$ e aplicando a condição $(d)$ para $i=0,1$,

$$
c_{i+1}=c_{i}+3 d_{i} h_{i}
$$

Isolando em (5.28) $d_{i}$ e subtituindo nas equações (5.25) e (5.27), derivam-se, para $i=0,1$, as equações

$$
a_{i+1}=a_{i}+b_{i} h_{i}+\frac{h_{i}^{2}}{3}\left(2 c_{i}+c_{i+1}\right)
$$

e 


$$
b_{i+1}=b_{i}+h_{i}\left(c_{i}+c_{i+1}\right) .
$$

Da equação (5.29) encontra-se $b_{i}$ como

$$
b_{i}=\frac{1}{h_{i}}\left(a_{i+1}-a_{i}\right)-\frac{h_{i}}{3}\left(2 c_{i}+c_{i+1}\right),
$$

ou, por uma redução dos índices, como

$$
b_{i-1}=\frac{1}{h_{i-1}}\left(a_{i}-a_{i-1}\right)-\frac{h_{i-1}}{3}\left(2 c_{i-1}+c_{i}\right) .
$$

Sustraindo a equação (5.31) da equação (5.32) e utilizando a equação (5.30), deriva-se o seguinte sistema linear nas variáveis $c_{0}, c_{1}$ e $c_{2}$

$$
h_{i-1} c_{i-1}+2\left(h_{i-1}+h_{i}\right) c_{i}+h_{i} c_{i+1}=\frac{3}{h_{i}}\left(a_{i+1}-a_{i}\right)-\frac{3}{h_{i-1}}\left(a_{i}-a_{i-1}\right), \quad i=1 .
$$

Determinada a solução desse sistema, obtém-se os coeficientes $b_{0}, b_{1}, b_{2}$ e $d_{0}, d_{1}, d_{2}$ a partir das equações (5.28) e (5.31), respectivamente. E assim determinam-se os polinônmios cúbicos $\left.S_{h}\right|_{I_{o}}(x)$ e $\left.S_{h}\right|_{I_{1}}(x)$ via a equação (5.23).

Mostra-se agora que o sistema (5.33) tem solução única, isto é se $S_{h}$ satisfaz as condições adicionais $(e)$ impostas como $S_{h}^{\prime}(0)=g^{\prime}(0)=1$ e $S_{h}^{\prime}(1)=g^{\prime}(1)=1$ (ver Lin e Chieng (1991) [44]), onde $g$, definida em $[0,1]$, é diferenciavel em 0 e 1 , então $g$ tem um único spline interpolador sobre $x_{0}=0, x_{1}=1 / 2, x_{2}=1$. De fato, aplicando a equação (5.31) com $i=0$ e lembrando que $g^{\prime}(0)=S_{h}^{\prime}(0)=S_{h}^{\prime}\left(x_{0}\right)=b_{0}$ obtém-se

$$
g^{\prime}(0)=\frac{1}{h_{0}}\left(a_{1}-a_{0}\right)-\frac{h_{0}}{3}\left(2 c_{0}+c_{1}\right)
$$

e consequentemente,

$$
2 h_{0} c_{0}+h_{0} c_{1}=\frac{3}{h_{0}}\left(a_{1}-a_{0}\right)-3 g^{\prime}(0) .
$$

De maneira similar, a partir da equação (5.30) resulta

$$
g^{\prime}(1)=b_{2}=b_{1}+h_{1}\left(c_{1}+c_{2}\right),
$$

de maneira que a equação (5.31) com $i=1$ fornece

$$
\begin{aligned}
g^{\prime}(1) & =\frac{a_{2}-a_{1}}{h_{1}}-\frac{h_{1}}{3}\left(2 c_{1}+c_{2}\right)+h_{1}\left(c_{1}+c_{2}\right), \\
& =\frac{a_{2}-a_{1}}{h_{1}}+\frac{h_{1}}{3}\left(c_{1}+2 c_{2}\right),
\end{aligned}
$$


$\mathrm{ou}$

$$
h_{1} c_{1}+2 h_{1} c_{2}=3 g^{\prime}(1)-\frac{3}{h_{1}}\left(a_{2}-a_{1}\right) .
$$

A equação (5.33), juntamente com as equações (5.35) e (5.37), resulta no sistema linear

$$
\left[\begin{array}{ccc}
2 h_{0} & h_{0} & 0 \\
h_{0} & 2\left(h_{0}+h_{1}\right) & h_{1} \\
0 & h_{1} & 2 h_{1}
\end{array}\right]\left[\begin{array}{c}
c_{0} \\
c_{1} \\
c_{2}
\end{array}\right]=\left[\begin{array}{c}
\frac{3\left(a_{1}-a_{0}\right)}{h_{0}}-3 g^{\prime}(0) \\
\frac{3\left(a_{2}-a_{1}\right)}{h_{1}}-\frac{3\left(a_{1}-a_{0}\right)}{h_{0}} \\
3 g^{\prime}(1)-\frac{3\left(a_{2}-a_{1}\right)}{h_{1}}
\end{array}\right]
$$

ou seja

$$
\left[\begin{array}{ccc}
1 & 1 / 2 & 0 \\
1 / 2 & 2 & 1 / 2 \\
0 & 1 / 2 & 1
\end{array}\right]\left[\begin{array}{c}
c_{0} \\
c_{1} \\
c_{2}
\end{array}\right]=\left[\begin{array}{c}
3 / 2 \\
-3 \\
3 / 2
\end{array}\right]
$$

Como a matriz dos coeficientes deste sistema é (estritamente) diagonal dominante $\left(\left|a_{i i}\right|>\right.$ $\sum_{j \neq i}\left|a_{i j}\right|$ ), pode-se garantir existencia e unicidade das soluções $c_{0}, c_{1}$ e $c_{2}$ (ver por exemplo, Quarterony (2007) [51]). A solução para este sistema linear é $c_{0}=3, c_{1}=-3$ e $c_{2}=3$. Portanto, a partir das equações $(5.28)$ e (5.31), obtém-se $b_{0}=1$ e $d_{0}=-4$. Fazendo $x=\hat{\phi}_{U}$, o spline $\left.S_{h}\right|_{I_{0}}$ é então dado como

$$
\left.S_{h}\right|_{I_{0}}\left(\hat{\phi}_{U}\right)=\hat{\phi}_{U}+3 \hat{\phi}_{U}^{2}-4 \hat{\phi}_{U}^{3}, \quad \hat{\phi}_{U} \in[0,1 / 2] .
$$

Fazendo $i=1$ e $\hat{\phi}_{U 1}=1 / 2$ na equação (5.23), tem-se

$$
\left.S_{h}\right|_{I_{1}}\left(\hat{\phi}_{U}\right)=a_{1}+b_{1}\left(\hat{\phi}_{U}-1 / 2\right)+c_{1}\left(\hat{\phi}_{U}-1 / 2\right)^{2}+d_{1}\left(\hat{\phi}_{U}-1 / 2\right)^{3},
$$

e levando em conta as equações (5.24), (5.28) e (5.31), determinam-se $a_{1}=3 / 4, b_{1}=1$ e $d_{1}=4$; $\left.S_{h}\right|_{I_{1}}\left(\hat{\phi}_{U}\right)$ via a equação $(5.22)$ é então dado como

$$
\left.S_{h}\right|_{I_{1}}\left(\hat{\phi}_{U}\right)=4 \hat{\phi}_{U}^{3}-9 \hat{\phi}_{U}^{2}+7 \hat{\phi}_{U}-1, \quad \hat{\phi}_{U} \in[1 / 2,1]
$$

A função (definida por duas sentenças) desejada é

$$
S_{h}\left(\hat{\phi}_{U}\right)=\left\{\begin{array}{lll}
-4 \hat{\phi}_{U}^{3}+3 \hat{\phi}_{U}^{2}+\hat{\phi}_{U}, & \text { se } & \hat{\phi}_{U} \in[0,1 / 2] \\
4 \hat{\phi}_{U}^{3}-9 \hat{\phi}_{U}^{2}+7 \hat{\phi}_{U}-1, & \text { se } & \hat{\phi}_{U} \in[1 / 2,1] .
\end{array}\right.
$$

Em resumo, o novo esquema convectivo CSPUS em variáveis normalizadas para o cálculo dos 
fluxos numéricos em uma face $f$ de uma célula computacional é definido por

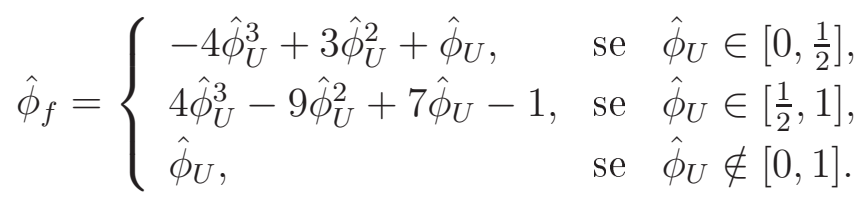

A figura 5.3 mostra o esquema CSPUS no plano $\hat{\phi}_{U} \perp \hat{\phi}_{f}$ dentro da região TVD.

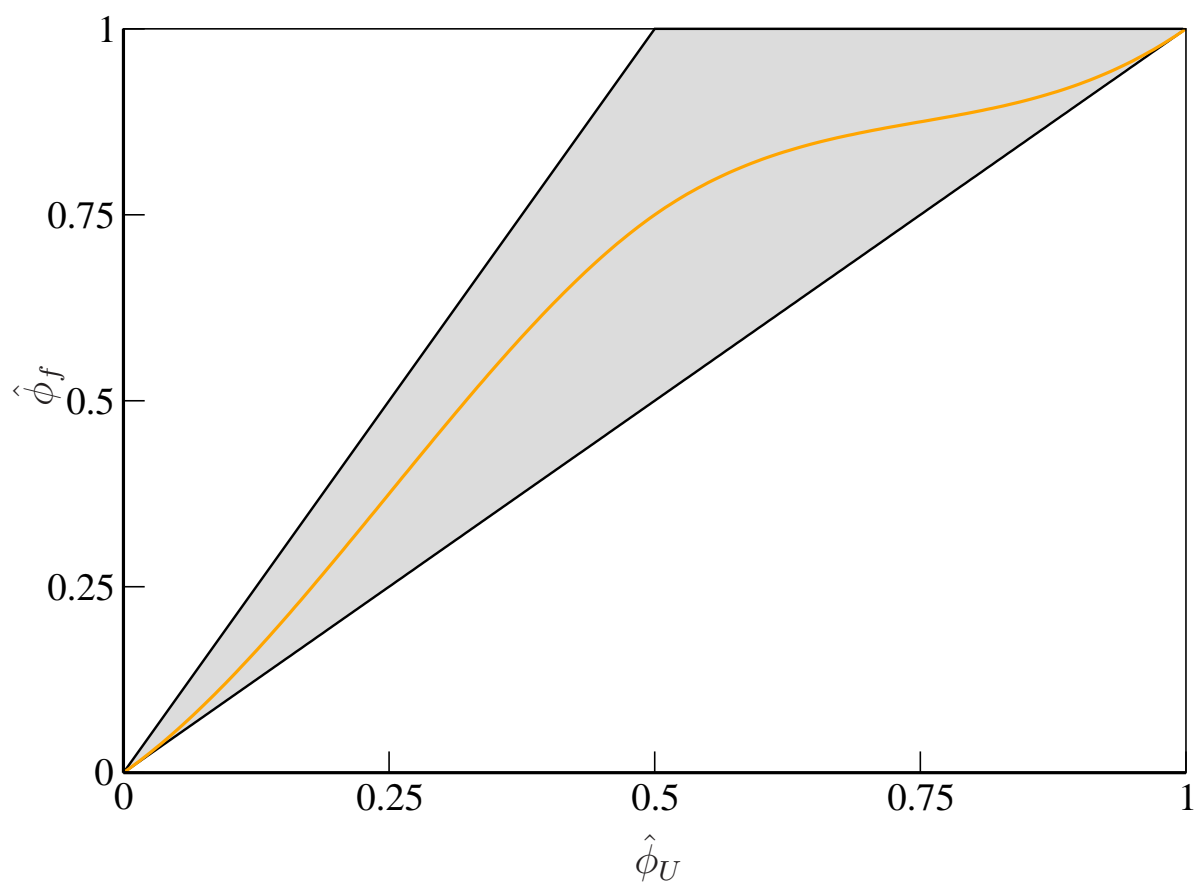

Figura 5.3: O esquema CSPUS em variáveis normalizadas dentro da região TVD.

Para obter o esquema CSPUS em variaveis não normalizadas aplicou-se a transformação (3.2), cujo resultado é

$$
\hat{\phi}_{f}=\left\{\begin{array}{lll}
\phi_{R}+\left(\phi_{D}-\phi_{R}\right)\left(-4 \hat{\phi}_{U}^{3}+3 \hat{\phi}_{U}^{2}+\hat{\phi}_{U}\right), & \text { se } & \hat{\phi}_{U} \in\left[0, \frac{1}{2}\right] \\
\phi_{R}+\left(\phi_{D}-\phi_{R}\right)\left(4 \hat{\phi}_{U}^{3}-9 \hat{\phi}_{U}^{2}+7 \hat{\phi}_{U}-1\right), & \text { se } & \hat{\phi}_{U} \in\left[\frac{1}{2}, 1\right] \\
\hat{\phi}_{U}, & \text { se } & \hat{\phi}_{U} \notin[0,1]
\end{array}\right.
$$

O limitador de fluxo correspondente ao esquema CSPUS, $\varphi\left(r_{f}\right)$, é obtido usando-se o método definido em (5.13) com $\hat{\phi}_{f}$ dado pela equação (5.43), cujas passagens são como segue:

-para $\hat{\phi}_{U} \in\left[0, \frac{1}{2}\right]$ 


$$
\begin{array}{r}
-4 \hat{\phi}_{U}^{3}+3 \hat{\phi}_{U}^{2}+\hat{\phi}_{U}=\hat{\phi}_{U}+\frac{1}{2} \varphi(r)\left(1-\hat{\phi}_{U}\right), \\
-4\left(\frac{r_{f}}{1+r_{f}}\right)^{3}+3\left(\frac{r_{f}}{1+r_{f}}\right)^{2}=\frac{1}{2} \varphi\left(r_{f}\right)\left(1-\frac{r_{f}}{1+r_{f}}\right),
\end{array}
$$

ou

$$
\varphi\left(r_{f}\right)=\frac{-2 r_{f}^{3}+6 r_{f}^{2}}{\left(1+r_{f}\right)^{2}} .
$$

$-\operatorname{para} \hat{\phi}_{U} \in\left[\frac{1}{2}, 1\right]$

$$
\begin{gathered}
4 \hat{\phi}_{U}^{3}-9 \hat{\phi}_{U}^{2}+7 \hat{\phi}_{U}-1=\hat{\phi}_{U}+\frac{1}{2} \varphi\left(r_{f}\right)\left(1-\hat{\phi}_{U}\right), \\
4\left(\frac{r_{f}}{1+r_{f}}\right)^{3}-9\left(\frac{r_{f}}{1+r_{f}}\right)^{2}+7\left(\frac{r_{f}}{1+r_{f}}\right)-1=\frac{r_{f}}{1+r_{f}}+\frac{1}{2} \varphi\left(r_{f}\right)\left(1-\frac{r_{f}}{1+r_{f}}\right),
\end{gathered}
$$

$\mathrm{ou}$

$$
\varphi\left(r_{f}\right)=\frac{6 r_{f}-2}{\left(1+r_{f}\right)^{2}}
$$

Em resumo, o novo limitador CSPUS é

$$
\varphi\left(r_{f}\right)= \begin{cases}\frac{-2 r_{f}^{3}+6 r_{f}{ }^{2}}{\left(1+r_{f}\right)^{2}}, & 0 \leq r_{f} \leq 1 \\ \frac{6 r_{f}-2}{\left(1+r_{f}\right)^{2}}, & r_{f} \geq 0 \\ 0, & r_{f}<0 .\end{cases}
$$

A figura 5.4 apresenta o gráfico do esquema CSPUS no plano $r_{f} \perp \varphi$, mostrando que ele é TVD.

\subsection{O Esquema RUS}

A idéia em derivar o esquema RUS foi escolher o limitador de fluxo correspondente como sendo uma função racional $\varphi\left(r_{f}\right)=P\left(r_{f}\right) / Q\left(r_{f}\right)$, com $P$ e $Q$ ambos de grau dois e $P(0)=0$ $(\varphi(0)=0)$, que atendesse (parcialmente) ao critério de positividade de [66] e que para $r_{f} \rightarrow$ $+\infty, \varphi\left(r_{f}\right) \rightarrow 1$ - LED-bounded (local extremum diminishing [49]). A função limitador de fluxo 


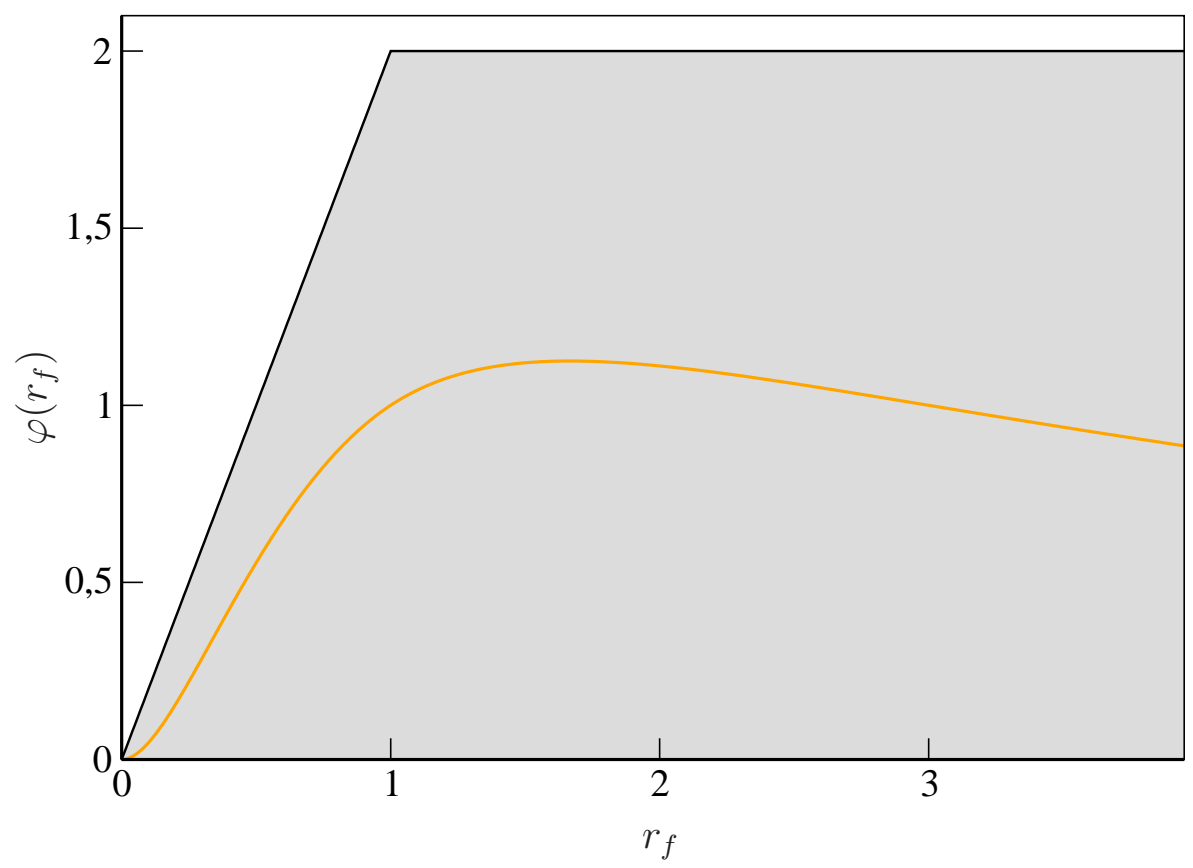

Figura 5.4: Limitador de fluxo correspondente ao esquema CSPUS na região TVD.

escolhida foi

$$
\varphi\left(r_{f}\right)=\frac{r_{f}^{2}+a r_{f}}{r_{f}^{2}+b r_{f}+c}, \quad \forall r_{f} \geq 0,
$$

onde impõe-se as condições (3.17) e (3.18), mais a imposição $\varphi\left(\frac{1}{2}\right)=\frac{3}{4}$, para se determinar os coeficientes $a, b$ e $c$. O resultado é o sistema não linear dado por

$$
\left\{\begin{array}{l}
a-b-c=0 \\
-4 a+2 b+6 c+4 a c-2 b c-b^{2}-c^{2}-1=0, \\
8 a-6 b-12 c+1=0,
\end{array}\right.
$$

cuja solução é

$$
a=1, \quad b=\frac{1}{2} \quad \text { e } \quad c=\frac{1}{2} .
$$

Em resumo, o limitador de fluxo correspondente ao esquema RUS é dado por

$$
\varphi\left(r_{f}\right)=\frac{r_{f}^{2}+r_{f}}{r_{f}^{2}+\frac{1}{2} r_{f}+\frac{1}{2}}, \quad \forall r_{f} \geq 0 .
$$

Vale observar que o limitador RUS satisfaz o princípio de monotonicidade de Sweby (1984) [67], isto é $\varphi^{\prime}(0)=2$. A figura 5.5 mostra para todo $r_{f}$ o limitador RUS no plano $r_{f} \perp \varphi$, evidenciando que ele satisfaz parcialmente a restrição de monoticidade de Spekreijse com $\alpha=0$ e $M=2$. 


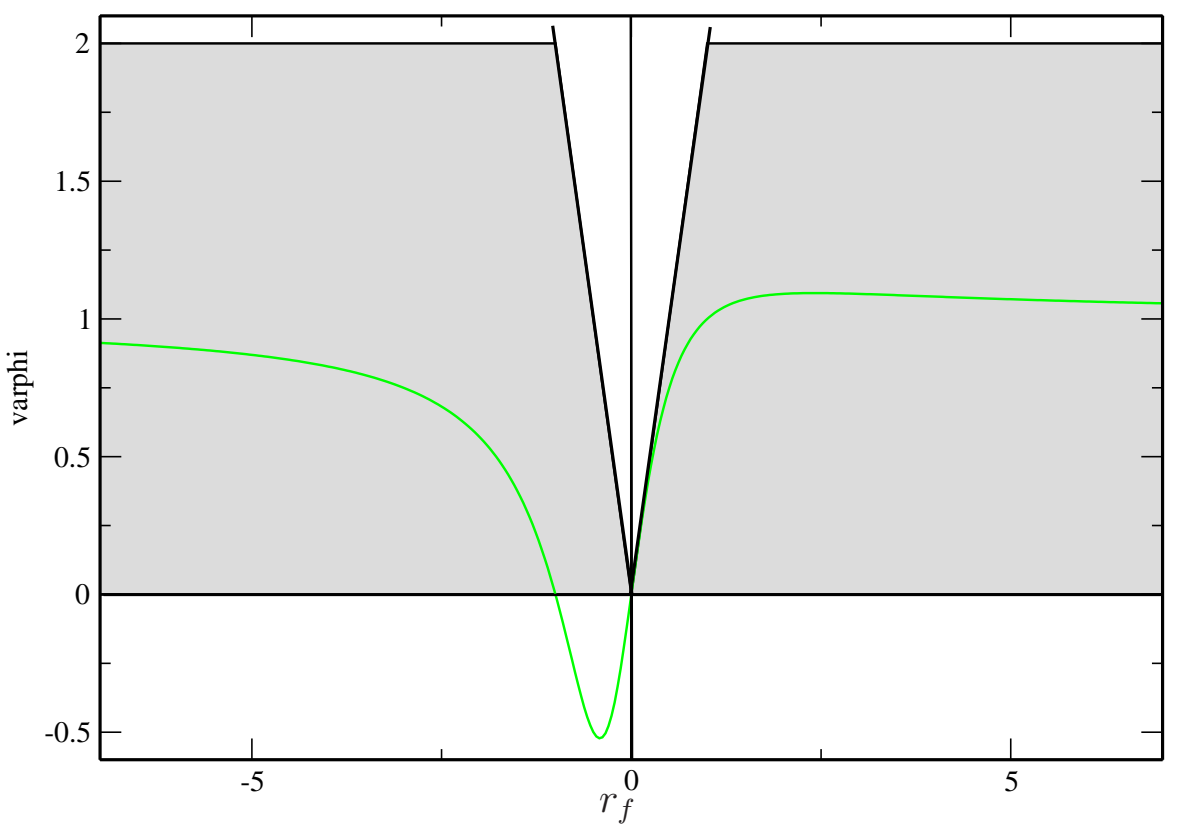

Figura 5.5: O limitador de fluxo RUS e a região de monoticidade de Spekreijse.

O esquema RUS, em variaveis normalizadas com $\hat{\phi}_{U} \in[0,1]$, é obtido fazendo o uso da equação (5.13) com a definição $r_{f}=\frac{\hat{\phi}_{U}}{1-\hat{\phi}_{U}}$ e com $\varphi\left(r_{f}\right)$ dado pela equação (5.50). O resultado é

$$
\hat{\phi}_{f}\left(\hat{\phi}_{U}\right)=\frac{2\left(\hat{\phi}_{U}^{3}-\hat{\phi}_{U}^{2}+\hat{\phi}_{U}\right)}{2 \hat{\phi}_{U}^{2}-\hat{\phi}_{U}+1}, \quad \hat{\phi}_{U} \in[0,1] .
$$

Atendendo as sugestões de Leonard (1988) [37], considerou-se o esquema FOU (Courant (1952) [14]) para $\hat{\phi}_{U} \notin[0,1]$, isto é, $\hat{\phi}_{f}=\hat{\phi}_{U}$. Em síntese, o esquema RUS é dado por

$$
\hat{\phi}_{f}=\left\{\begin{array}{lll}
\frac{2\left(\hat{\phi}_{U}^{3}-\hat{\phi}_{U}^{2}+\hat{\phi}_{U}\right)}{2 \hat{\phi}_{U}^{2}-\hat{\phi}_{U}+1}, & \text { se } \hat{\phi}_{U} \in[0,1] \\
\hat{\phi}_{U}, & \text { se } \hat{\phi}_{U} \notin[0,1]
\end{array}\right.
$$

Pode-se observar claramente que o esquema RUS verifica as condições de Leonard para derivar um esquema monotônico de alta ordem não-linear formulado em variáveis normalizadas (ver capítulo 3), as quais são: passar pelos pontos $O(0,0)$ e $P(1,1)$ (para o esquema ser monotônico), passar pelo ponto $Q\left(\frac{1}{2}, \frac{3}{4}\right)$ para atingir segunda ordem de precisão e passar pelo ponto $Q$ com inclinação $\frac{3}{4}$ para alcançar terceira ordem de precisão. Em variáveis não normalizadas o esquema RUS fica

$$
\phi_{f}=\left\{\begin{array}{lll}
\phi_{R}+\left(\phi_{D}-\phi_{R}\right) \frac{2\left(\hat{\phi}_{U}^{3}-\hat{\phi}_{U}^{2}+\hat{\phi}_{U}\right)}{2 \hat{\phi}_{U}^{2}-\hat{\phi}_{U}+1}, & \text { se } & \hat{\phi}_{U} \in[0,1] \\
\phi_{U}, & \text { se } & \hat{\phi}_{U} \notin[0,1]
\end{array}\right.
$$


A figura 5.6 mostra o esquema RUS no plano $\hat{\phi}_{U} \perp \hat{\phi}_{f}$ na região TVD.

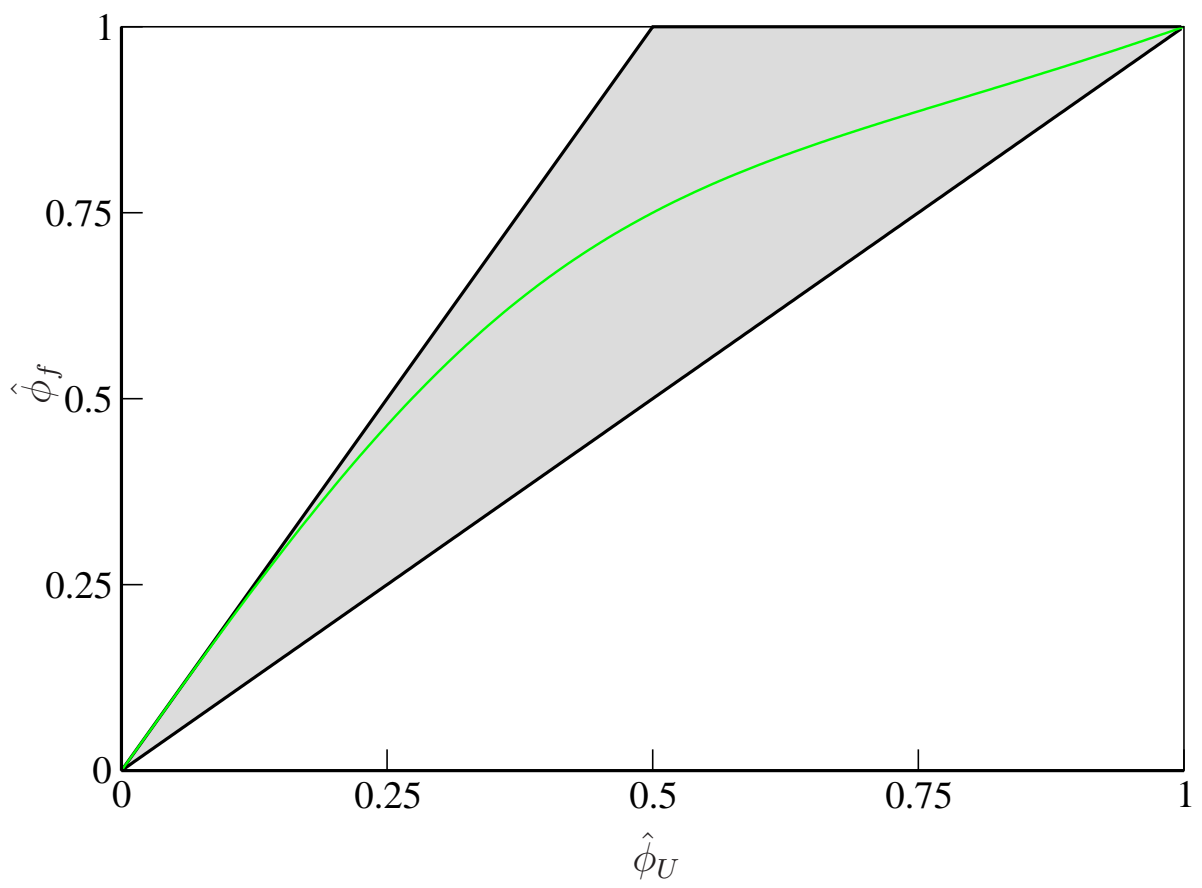

Figura 5.6: O esquema RUS dentro da região TVD.

Uma modificação simples do esquema original RUS pode ser obtida introduzindo-se uma constante positiva $\varepsilon$ na função limitadora (5.50). Esta modificação está sendo explorada neste trabalho com o objetivo de prevenir oscilações não físicas em regiões de gradientes elevados e descontinuidades, melhorar a convergência numérica global e aliviar o aparecimento de extremos (picos e vales) suaves nas soluções computadas. A modificação é descrita como segue. Seja a variavel $r_{f}$ definida em termos da razão dos gradientes $a=\left(\frac{\partial \phi}{\partial x}\right)_{f}$ e $b=\left(\frac{\partial \phi}{\partial x}\right)_{g}$, como definidos na equação (3.13). Em função de $a$ e $b$, o limitador (5.50) é escrito como

$$
\varphi\left(r_{f}\right)=\varphi(b / a)=\frac{a b^{2}+b a^{2}}{a b^{2}+\frac{1}{2} b a^{2}+\frac{1}{2} a^{3}},
$$

e procedendo de maneira similar como feito por van Albada em [75], introduz-se a constante $\epsilon>0$ na formulação (5.54) para obter

$$
\varphi(b / a)=\frac{a\left(b^{2}+\epsilon\right)+b\left(a^{2}+\epsilon\right)}{a\left(b^{2}+\frac{1}{2} b a+\frac{1}{2} a^{2}+2 \epsilon\right)} .
$$




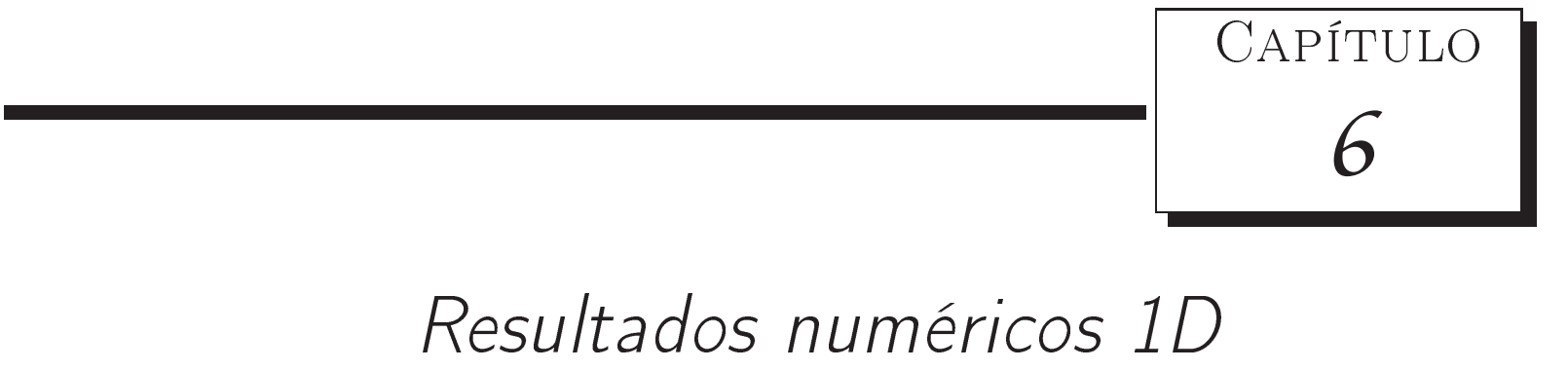

A finalidade desse capítulo é investigar o comportamento dos esquemas de alta resolução FDHERPUS, CSPUS, RUS e MRUS em problemas de interesse em fluidodinâmica computacional, como transporte de escalares, equação de Burgers, equações de Euler e águas rasas. Resultados numéricos obtidos com esses esquemas são comparados com outros resultados de esquemas consolidados na literatura, tais como van Albada (1982) [75], MC (Monotonized Central-Difference) proposto por van Leer (1997) [79], Minmod proposto por Roe (1982) [61]. Além disso, analisa-se o conceito de estabilidade TVD e calculam-se estimativas para a ordem de convergência dos métodos numéricos globais equipados com os esquemas convectivos propostos neste trabalho.

\subsection{Equação linear de advecção}

Nesta seção são apresentados resultados numéricos, considerando condições iniciais suaves e não-suaves, para o caso da equação escalar (linear) de advecção (2.3), a qual descreve o transporte da variável $u$ ao longo do eixo $x$ com velocidade de convecção $a=1$.

\section{Teste 1: Condição inicial suave}

A condição inicial suave considerada aqui é definida como

$$
u_{0}(x)=e^{-10(x+1)^{2}}, \quad x \in[-2,2] .
$$

Foram utilizados nos cálculos 200 células computacionais, número de Courant $C=0.8$ e tempo final de simulação $t=1.0$. A Figura 6.1 mostra os resultados obtidos com os esquemas van Albada, FDHERPUS, CSPUS, RUS e MRUS com parâmetros $\epsilon=10^{-7}$ e $\epsilon=10^{-8}$, mais a solução exata do problema. Observa-se por essa Figura (caso (a)) que, no global, todos os 
esquemas forneceram (praticamente) a mesma solução. Em particular nesta Figura (caso (b)), observa-se que o esquema MRUS, $\operatorname{com} \epsilon=10^{-7}$ resolveu melhor o problema nas vizinhanças do ponto de máximo, principalmente quando aumentou-se o número de células computacionais no domínio; isso pode ser confirmado na Tabela 6.1 onde constam os erros relativos $E_{h}$ na norma $L_{2}$ e as ordens locais de convergência $q$. Estes dados estão também graficados na Figura 7.2.

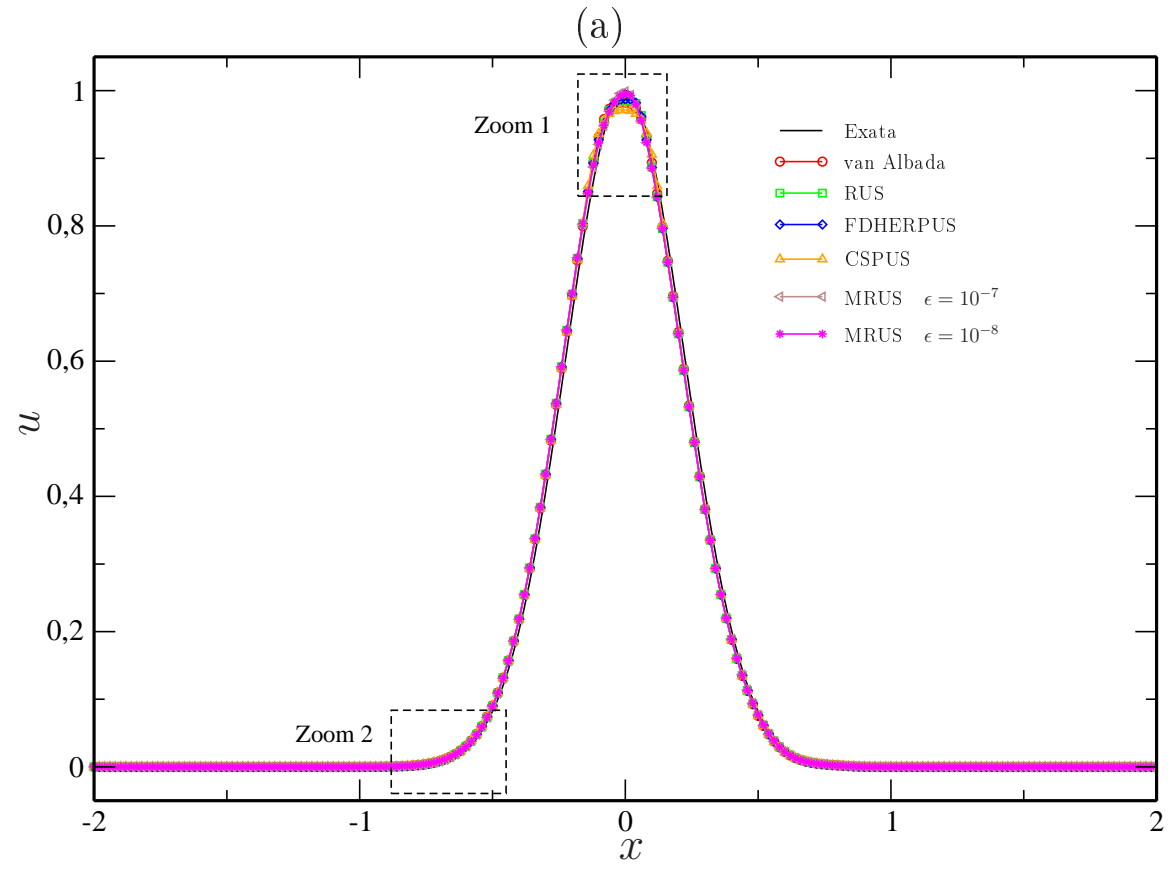

(b)

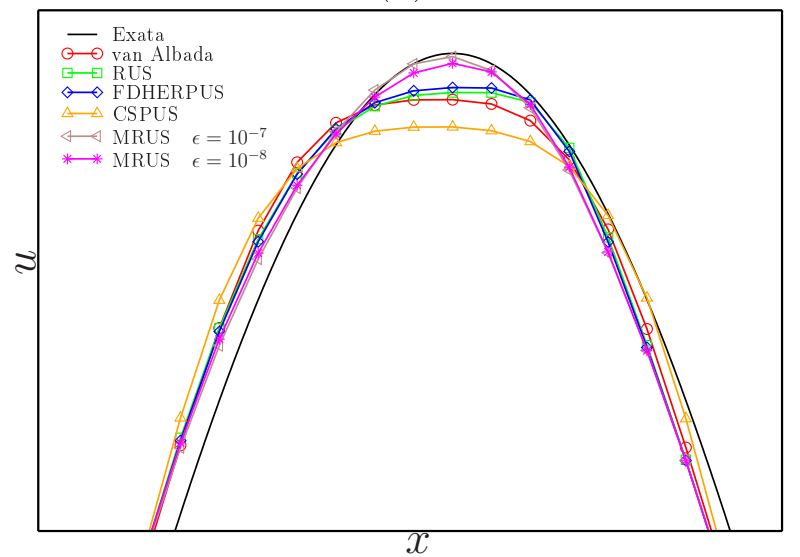

(c)

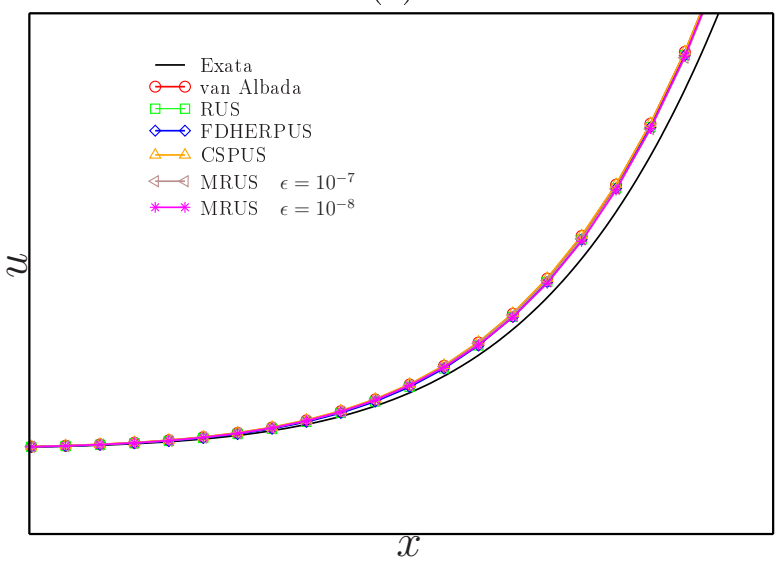

Figura 6.1: Soluções exata e numéricas para a equação linear de advecção: (a) aproximações obtidas pelos esquemas: van Albada, RUS, FDHERPUS, CSPUS, MRUS para $\epsilon=10^{-7} \mathrm{e}$ $\epsilon=10^{-8}$, (b) e (c) detalhes do comportamento dos esquemas em regiões específicas. 
Tabela 6.1: Erro relativo $E_{h}$ e estimativa para a ordem de convergência $q$.

\begin{tabular}{|c|c|c|c|}
\hline \multirow[t]{2}{*}{ Esquema } & \multirow[t]{2}{*}{ Malha } & \multicolumn{2}{|c|}{$L_{2}$} \\
\hline & & Erro & Ordem \\
\hline \multirow[t]{6}{*}{ van Albada } & 20 & 0.182719 & - \\
\hline & 40 & 0.045003 & 2.050157 \\
\hline & 80 & 0.009449 & 2.021534 \\
\hline & 160 & 0.001962 & 2.251788 \\
\hline & 320 & 0.000439 & 2.267837 \\
\hline & 640 & 0.000106 & 2.160032 \\
\hline \multirow[t]{6}{*}{ FDHERPUS } & 20 & 0.182734 & - \\
\hline & 40 & 0.043645 & 2.021480 \\
\hline & 80 & 0.008646 & 2.065857 \\
\hline & 160 & 0.001761 & 2.335712 \\
\hline & 320 & 0.000406 & 2.295638 \\
\hline & 640 & 0.000100 & 2.116843 \\
\hline \multirow[t]{6}{*}{ CSPUS } & 20 & 0.243889 & - \\
\hline & 40 & 0.081926 & 2.192317 \\
\hline & 80 & 0.017425 & 1.973831 \\
\hline & 160 & 0.003402 & 1.933163 \\
\hline & 320 & 0.000588 & 2.156704 \\
\hline & 640 & 0.000112 & 2.232495 \\
\hline \multirow[t]{6}{*}{ RUS } & 20 & 0.165893 & - \\
\hline & 40 & 0.039795 & 2.040642 \\
\hline & 80 & 0.008580 & 2.059594 \\
\hline & 160 & 0.001870 & 2.213538 \\
\hline & 320 & 0.000432 & 2.197939 \\
\hline & 640 & 0.000105 & 2.113935 \\
\hline \multirow[t]{6}{*}{ MRUS $\epsilon=10^{-7}$} & 20 & 0.181352 & - \\
\hline & 40 & 0.041741 & 1.996277 \\
\hline & 80 & 0.008039 & 2.119256 \\
\hline & 160 & 0.001669 & 2.376377 \\
\hline & 320 & 0.000387 & 2.268032 \\
\hline & 640 & 0.000097 & 2.108578 \\
\hline \multirow[t]{6}{*}{ MRUS $\epsilon=10^{-8}$} & 20 & 0.185903 & - \\
\hline & 40 & 0.043000 & 2.000000 \\
\hline & 80 & 0.008202 & 2.112141 \\
\hline & 160 & 0.001710 & 2.390289 \\
\hline & 320 & 0.000396 & 2.261979 \\
\hline & 6453 & 0.000099 & 2.110424 \\
\hline
\end{tabular}




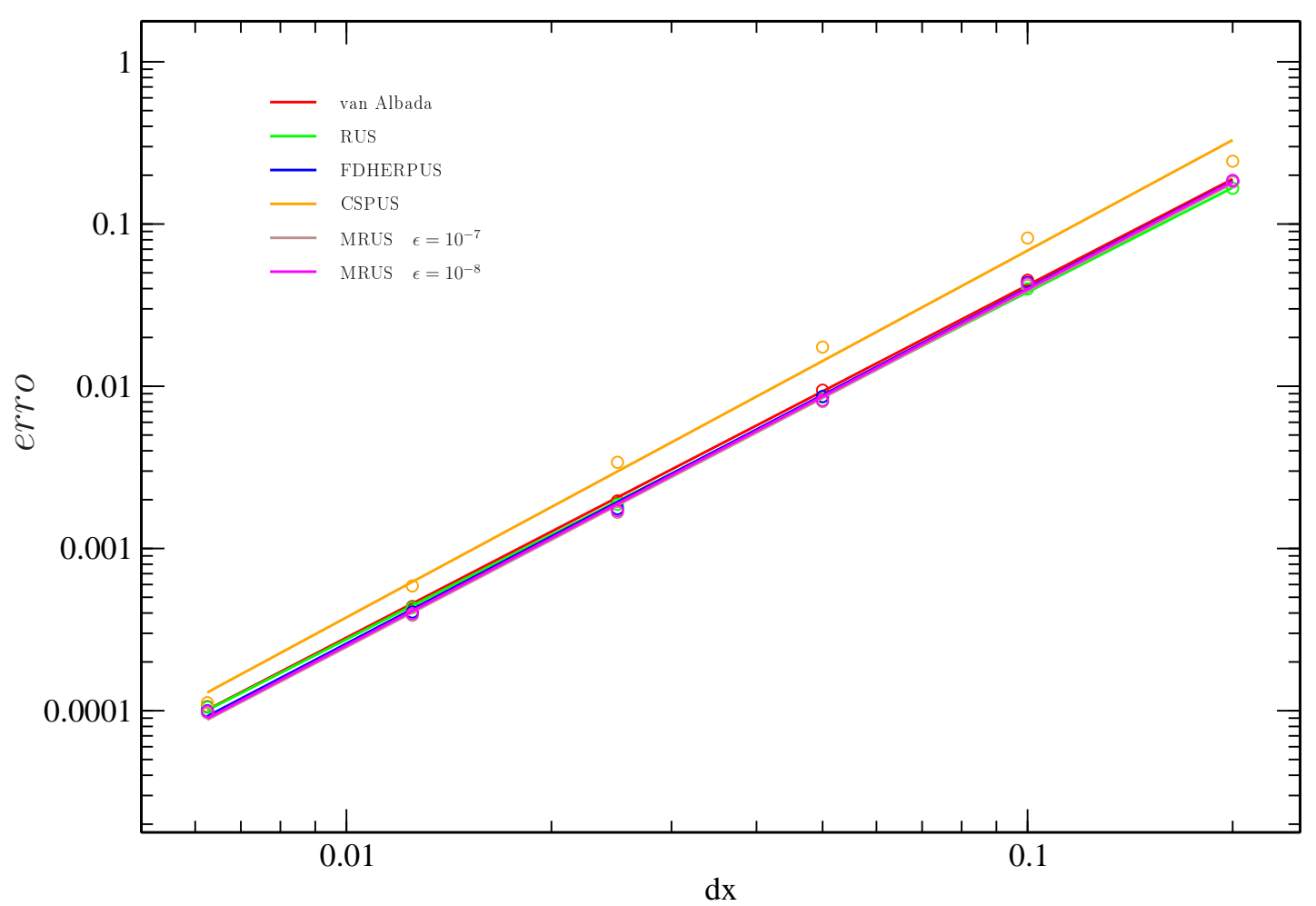

Figura 6.2: Erro na norma $L_{1}$ e na escala log-log para os esquemas van Albada, RUS, FDHERPUS, CSPUS e MRUS com $\epsilon=10^{-7}$ e $\epsilon=10^{-8}$. 


\section{Teste 2: Condição inicial não-suave}

Com o propósito de averiguar o desempenho dos esquemas convectivos FDHERPUS, CSPUS, RUS e MRUS em regiões de gradientes elevados, foi resolvido o problema de advecção escalar (2.3) para o caso de dados iniciais descontínuos (ver Harten (1989) [31]) definidos por

$$
u_{0}(x)= \begin{cases}-x \sin \left(\frac{3 \pi x^{2}}{2}\right), & -1 \leq x \leq-\frac{1}{3}, \\ |\sin (2 \pi x)|, & |x|<\frac{1}{3}, \\ 2 x-1-\frac{1}{6} \sin (3 \pi x), & \frac{1}{3} \leq x \leq 1 .\end{cases}
$$

Os dados utilizados na simulação foram: uma malha com 400 células computacionais, número de Courant $C=0.8$ e tempo final $t=0.5$. Na Figura 6.3 estão mostrados os resultados obtidos, bem como detalhes em regiões de máximos (picos) e mínimos (vales), com os esquemas van Albada, FDHERPUS, CSPUS, RUS e MRUS com $\epsilon=10^{-7}$ e $\epsilon=10^{-8}$.

Pode-se observar nesta Figura que foram observadas as mesmas informações obtidas no caso de dados iniciais suaves (Teste 1). Em particular, resultados (ligeiramente) melhores foram derivados com o esquema FDHERPUS; isto pode também ser visto na Tabela 6.2, onde está mostrado o erro relativo em diferentes malhas e uma estimativa para a ordem de convergência. Verifica-se também por essa Tabela que os novos esquemas mostram-se competitivos com o esquema consagrado de van Albada.

Para este mesmo problema foi feita ainda uma análise de restrição TVD para os esquemas van Albada, RUS, FDHERPUS e CSPUS, onde calcula-se a TV dada pela equação (3.7) em cada passo no tempo, considera-se uma malha fixa de 50 células computacionais e usa-se tempo final $t=0.5$. Isto está mostrado Figura 6.4, onde nota-se que as TV's não aumentam com o transcorrer do tempo. É importante observar neste ponto que as TV's para os esquemas MRUS com $\epsilon=10^{-7}$ e $\epsilon=10^{-8}$ (não mostradas na Figura 6.4) não decrescem ou aumentam com o tempo. 
(a)

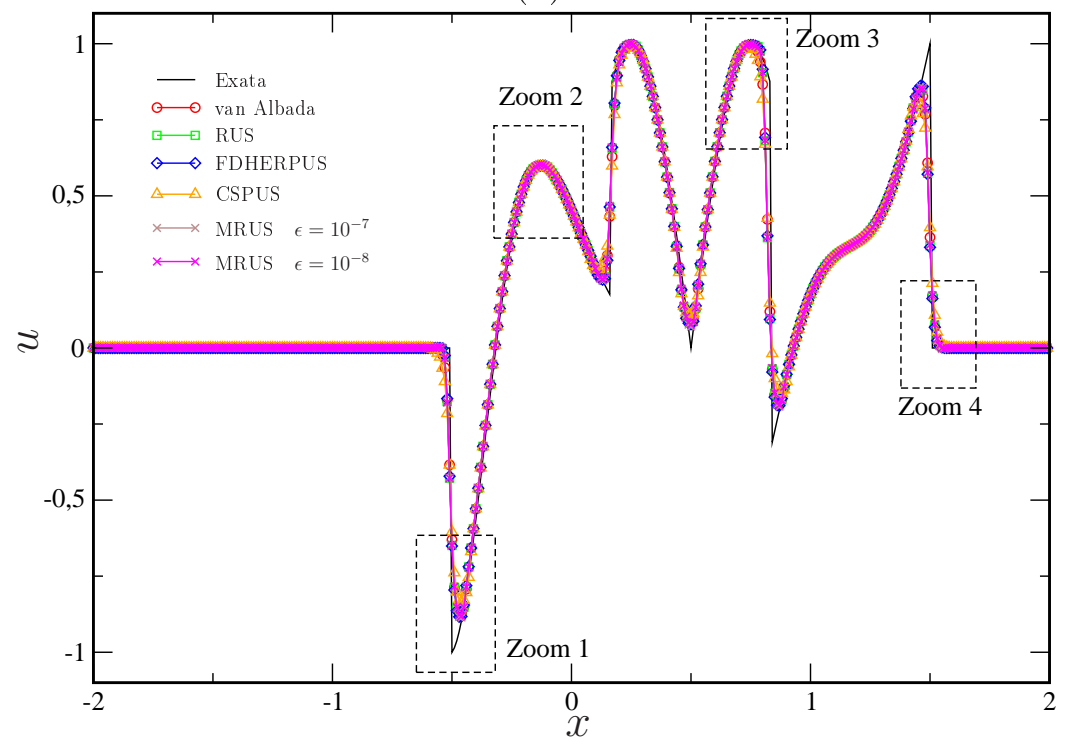

(b)

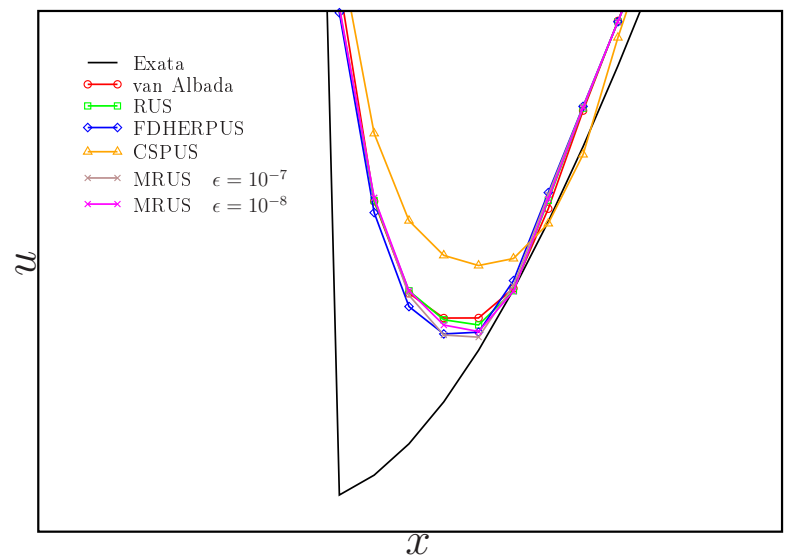

(d)

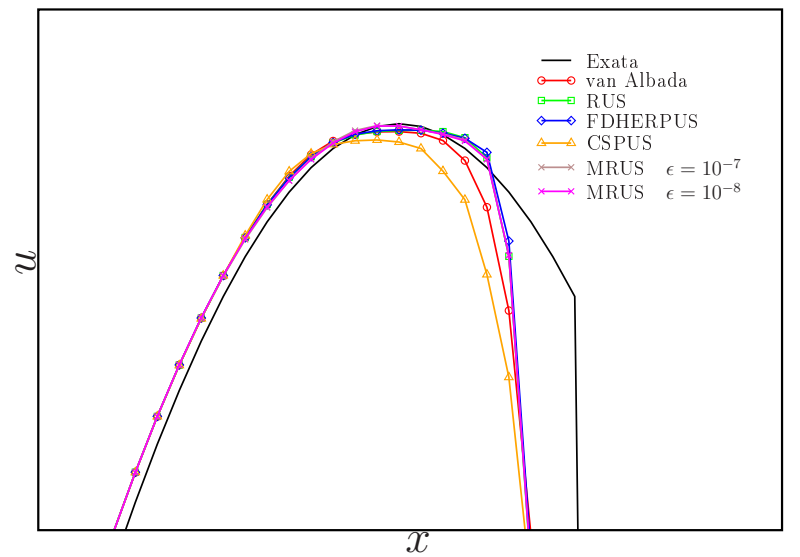

(c)

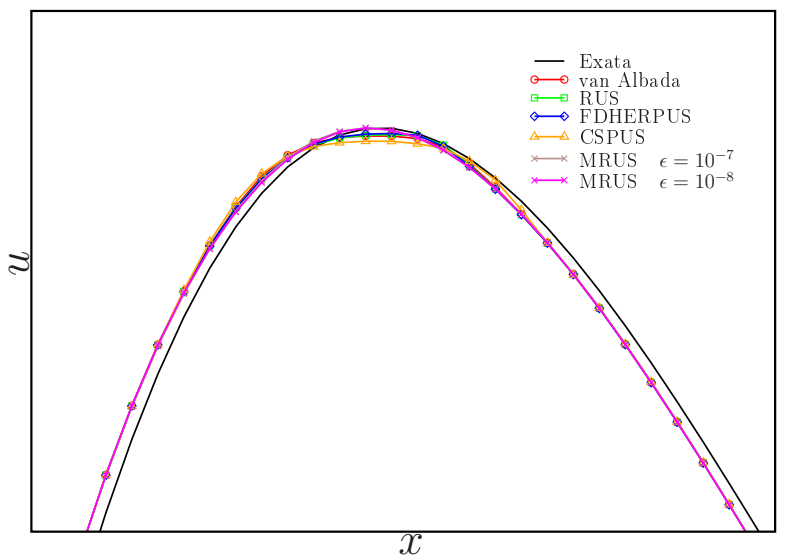

(e)

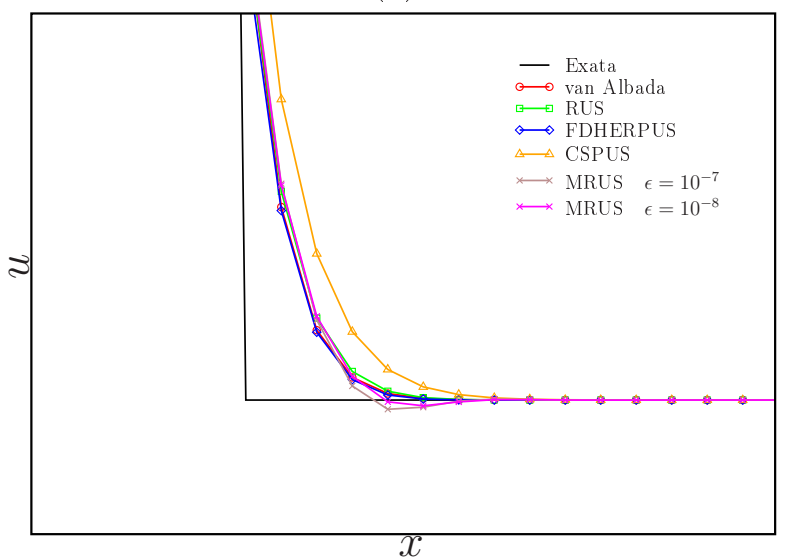

Figura 6.3: (a) Soluções exata e numéricas para a equação linear de advecção com condição inicial (6.2). As aproximações são obtidas pelos esquemas: van Albada, RUS, FDHERPUS, CSPUS, MRUS para $\epsilon=10^{-7}$ e $\epsilon=10^{-8}$. Detalhes das regiões demarcadas são mostrados em (b), (c), (d) e (e). 
Tabela 6.2: Erro relativo $E_{h}$ e estimativa para a ordem de convergência $q$.

\begin{tabular}{|c|c|c|c|c|c|}
\hline \multirow[t]{2}{*}{ Esquema } & \multirow[t]{2}{*}{ Malha } & \multicolumn{2}{|c|}{$L_{1}$} & \multicolumn{2}{|c|}{$L_{2}$} \\
\hline & & Erro & Ordem & Erro & Ordem \\
\hline \multirow{7}{*}{$\begin{array}{l}\text { van } \\
\text { Albada }\end{array}$} & & & & & \\
\hline & 20 & 0.81899 & - & 0.62018 & - \\
\hline & 40 & 0.56080 & 0.574399 & 0.57105 & 0.322755 \\
\hline & 80 & 0.34733 & 0.476512 & 0.37606 & 0.119088 \\
\hline & 160 & 0.21106 & 0.761029 & 0.28199 & 0.602657 \\
\hline & 320 & 0.12090 & 0.718607 & 0.20148 & 0.415283 \\
\hline & 640 & 0.08119 & 0.803882 & 0.16109 & 0.484986 \\
\hline \multirow[t]{6}{*}{$\overline{\mathrm{RUS}}$} & 20 & 0.80899 & - & 0.61508 & - \\
\hline & 40 & 0.58180 & 0.507119 & 0.56498 & 0.217629 \\
\hline & 80 & 0.32648 & 0.475586 & 0.36098 & 0.122578 \\
\hline & 160 & 0.19758 & 0.833520 & 0.27278 & 0.646269 \\
\hline & 320 & 0.11457 & 0.724517 & 0.19996 & 0.404186 \\
\hline & 640 & 0.08061 & 0.786202 & 0.17196 & 0.447983 \\
\hline \multirow[t]{6}{*}{ FDHERPUS } & 20 & 0.80807 & - & 0.61836 & - \\
\hline & 40 & 0.57001 & 0.475105 & 0.55417 & 0.201292 \\
\hline & 80 & 0.30455 & 0.503493 & 0.34465 & 0.158118 \\
\hline & 160 & 0.18491 & 0.904308 & 0.26254 & 0.685197 \\
\hline & 320 & 0.10863 & 0.719856 & 0.19435 & 0.392595 \\
\hline & 640 & 0.07815 & 0.767401 & 0.16904 & 0.433880 \\
\hline \multirow[t]{6}{*}{ CSPUS } & 20 & 0.81576 & - & 0.61229 & - \\
\hline & 40 & 0.62325 & 0.719752 & 0.60156 & 0.386472 \\
\hline & 80 & 0.43024 & 0.388334 & 0.43968 & 0.025506 \\
\hline & 160 & 0.28096 & 0.534669 & 0.33619 & 0.452255 \\
\hline & 320 & 0.16968 & 0.614777 & 0.24953 & 0.387177 \\
\hline & 640 & 0.10303 & 0.727548 & 0.19089 & 0.430064 \\
\hline \multirow{7}{*}{$\begin{array}{l}\text { MRUS } \\
\epsilon=10^{-7}\end{array}$} & & & & & \\
\hline & 20 & 0.81258 & - & 0.61647 & - \\
\hline & 40 & 0.58037 & 0.479067 & 0.56240 & 0.229802 \\
\hline & 80 & 0.32338 & 0.485537 & 0.35859 & 0.132434 \\
\hline & 160 & 0.19041 & 0.843742 & 0.27022 & 0.649261 \\
\hline & 320 & 0.10865 & 0.764121 & 0.19878 & 0.408201 \\
\hline & 640 & 0.07795 & 0.809421 & 0.16951 & 0.442962 \\
\hline \multirow[t]{6}{*}{$\begin{array}{l}\text { MRUS } \\
\epsilon=10^{-8}\end{array}$} & 20 & 0.81122 & 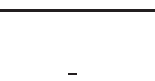 & 0.61584 & 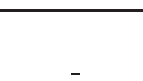 \\
\hline & 40 & 0.58120 & 0.502738 & 0.56378 & 0.227666 \\
\hline & 80 & 0.32512 & 0.481059 & 0.35998 & 0.127423 \\
\hline & 160 & 0.19456 & 0.838062 & 0.27201 & 0.647216 \\
\hline & 320 & 0.11134 & 0.740757 & 0.19961 & 0.404257 \\
\hline & 640 & 0.07858 & 0.805243 & 0.17047 & 0.446476 \\
\hline
\end{tabular}




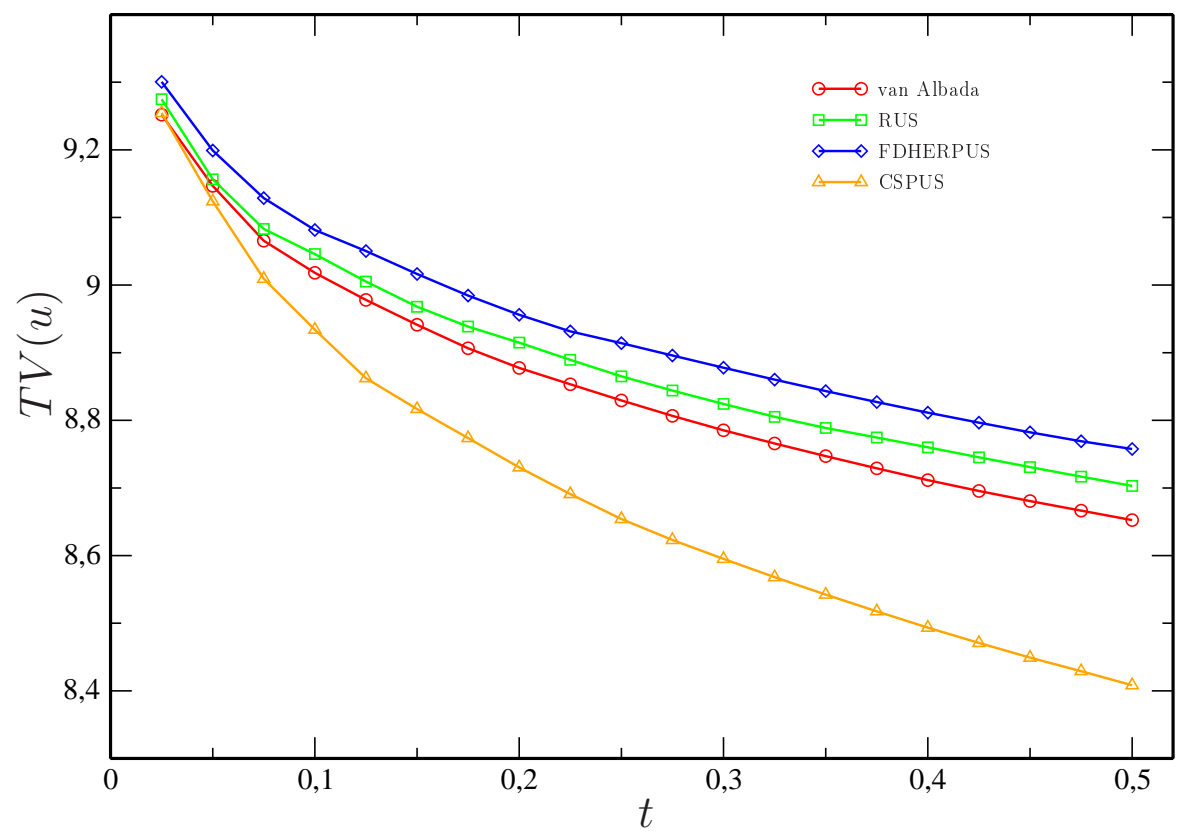

Figura 6.4: Variação total para o Teste 2 com respeito a tempo $t$.

\subsection{Equação não-linear de Burgers}

Nesta subseção será resolvida a equação não-linear de Burgers (2.6) para os casos viscoso $(\boldsymbol{S} \neq 0)$ e não-viscoso $(\boldsymbol{S}=0)$, além de uma comparação dos resultados numéricos obtidos com a solução exata do problema.

\section{Teste 3: Caso não-viscoso}

A equação de Burgers é suplementada com a condição inicial

$$
u_{0}(x)=1+\frac{1}{2} \sin (\pi x), \quad x \in[-1,1],
$$

e condições de contorno $u(-1, t)=u(1, t)$ (condições periódicas). O problema é um exemplo clássico para a validação de métodos numéricos para EDPs. A solução exata deste problema (ver Zhen (2009) [69]) é dada (implicitamente) como

$$
u(x, t)=1+\frac{1}{2} \sin (\pi(x-u t)) .
$$

A Figura 6.5 mostra a evolução no tempo $(0 \leq t \leq 1.6)$ da condição inicial (6.3) obtida com o limitador de fluxo FDHERPUS em uma malha de 400 células computacionais e a número de Courant $C=0.9$. 


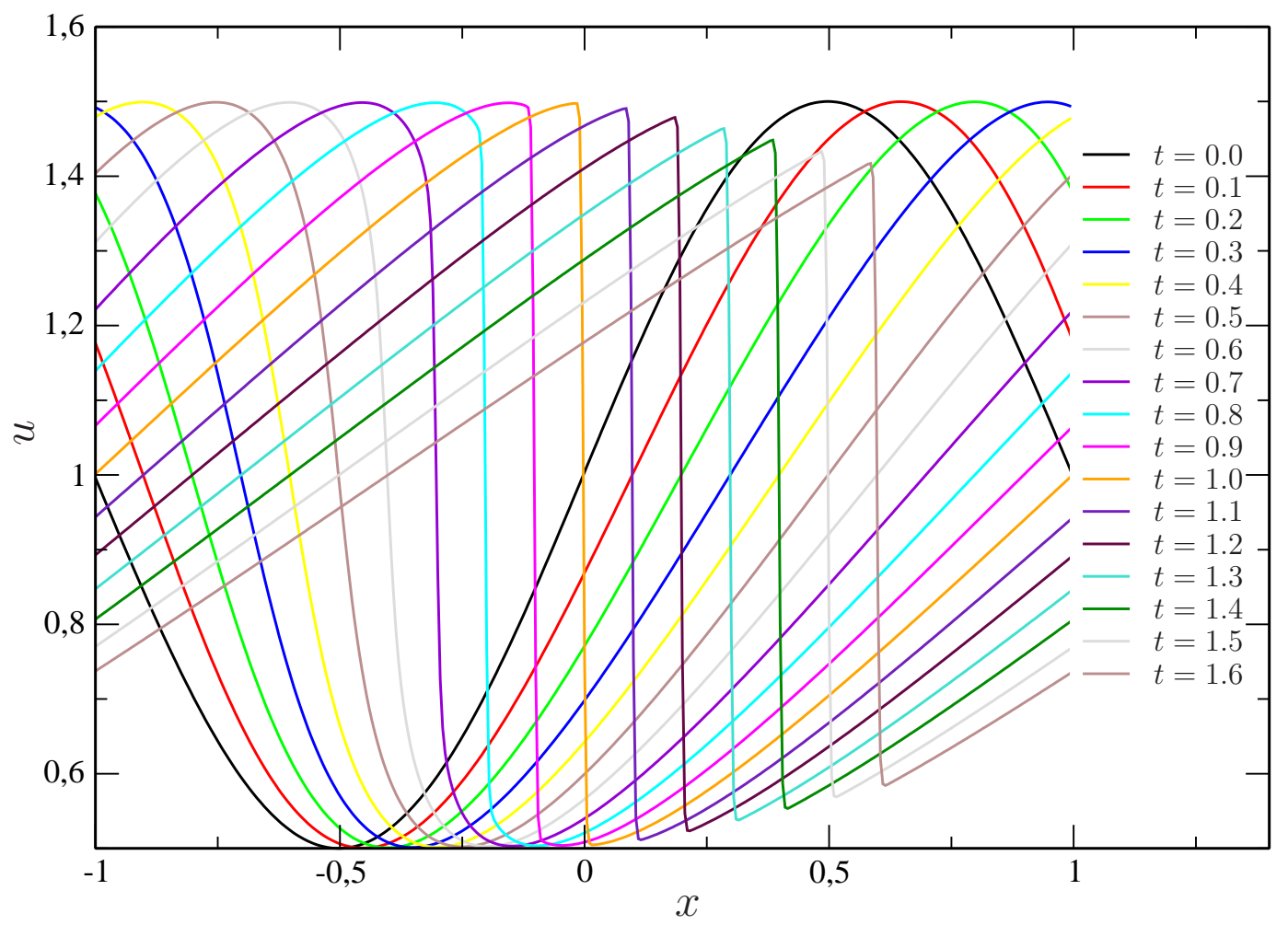

Figura 6.5: Evolução da condição inicial (6.3) ao longo do tempo para o limitador de fluxo correspondente ao esquema FDHERPUS.

Para mostrar o desempenho dos esquemas desenvolvidos neste trabalho na resolução desse problema não linear, é apresentado na Figura 6.6 uma comparação dos resultados obtidos com esses esquemas e os resultados produzidos pelo esquema de van Albada (1982) [75]. Os dados considerados na simulação são uma malha de 400 células computacionais, número de Courant $C=0.4$ e tempos finais $t=0.25$ (antes do aparecimento do choque) e $t=1.6$ (após o aparecimento do choque). É possível observar por essa Figura (no global - caso (a)) que os resultados foram bastante semelhantes. Observa-se ainda por essa mesma Figura que nas regiões suaves (caso (b) e caso (e)) todos os resultados obtidos são quase idênticos à solução exata. No ponto de descontinuidade $(x=0.5)$, os esquemas FDHERPUS e MRUS com $\epsilon=10^{-8}$ produziram (ligeiramente) os melhores resultados. A Tabela 6.3 contempla os erros na norma $L_{2}$ e as ordens de convergência observadas, os quais quantificam os resultados apresentados na Figura 6.6. 
(a)

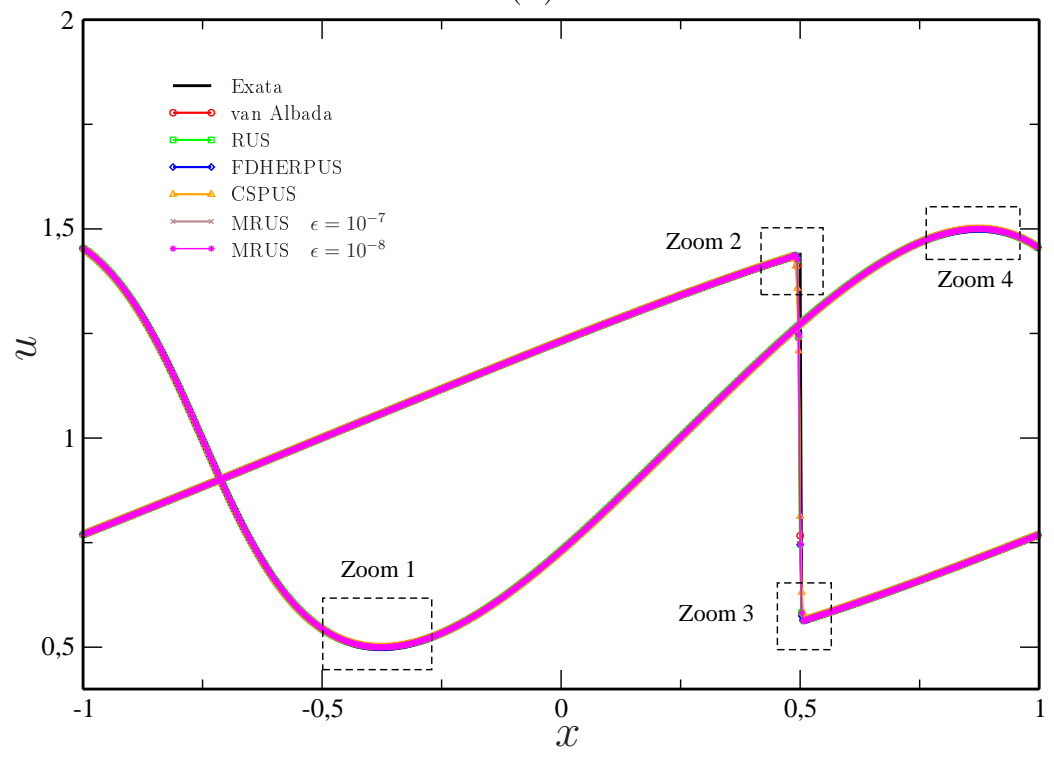

(b)

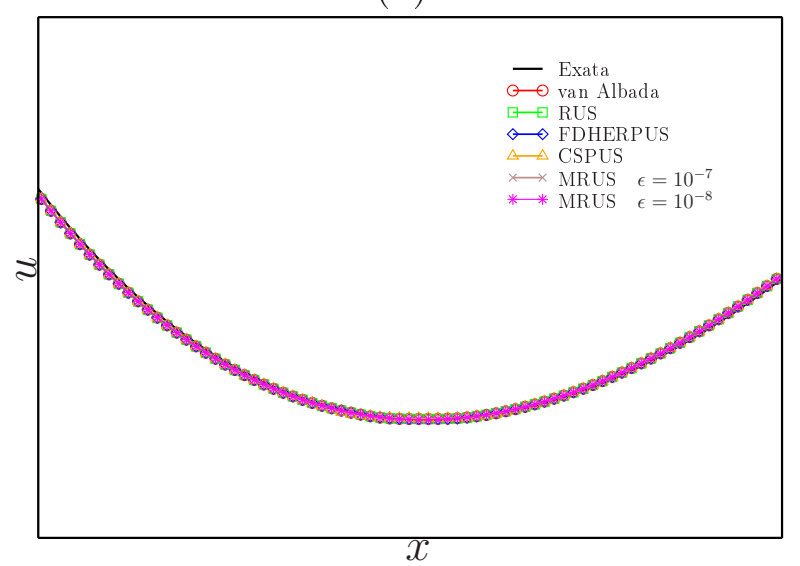

(c)

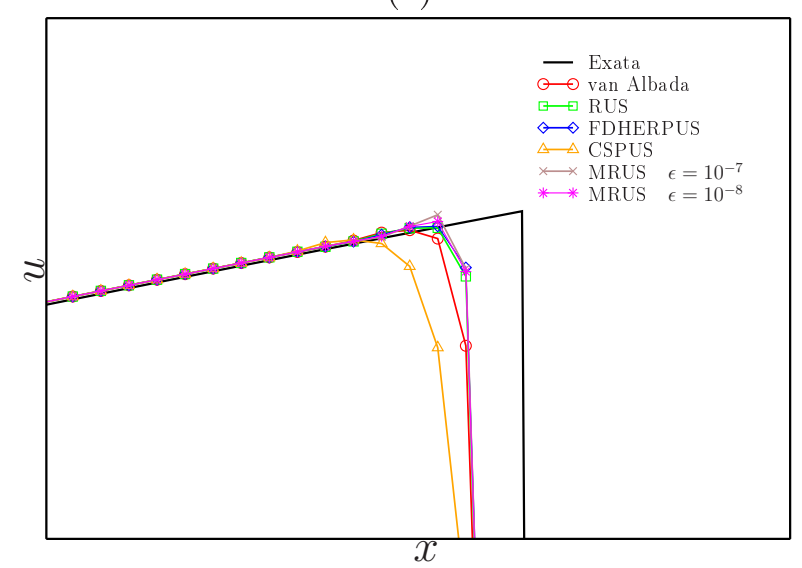

(d)

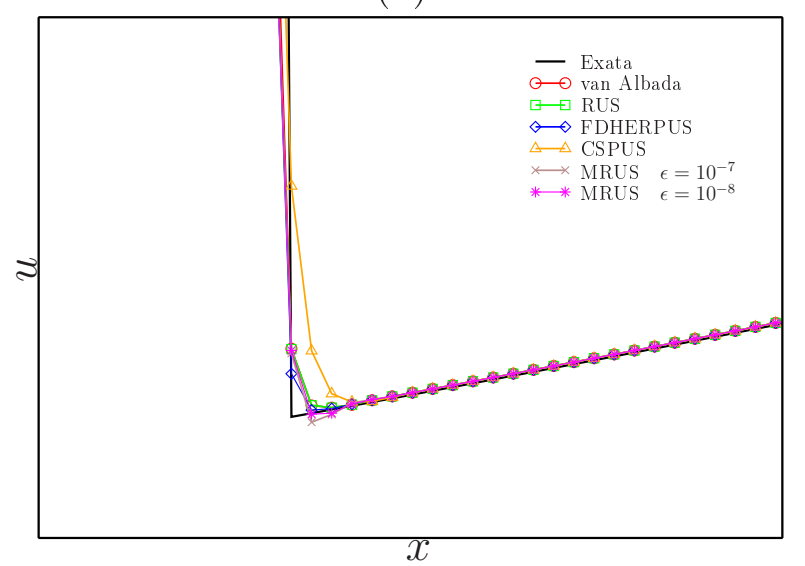

(e)

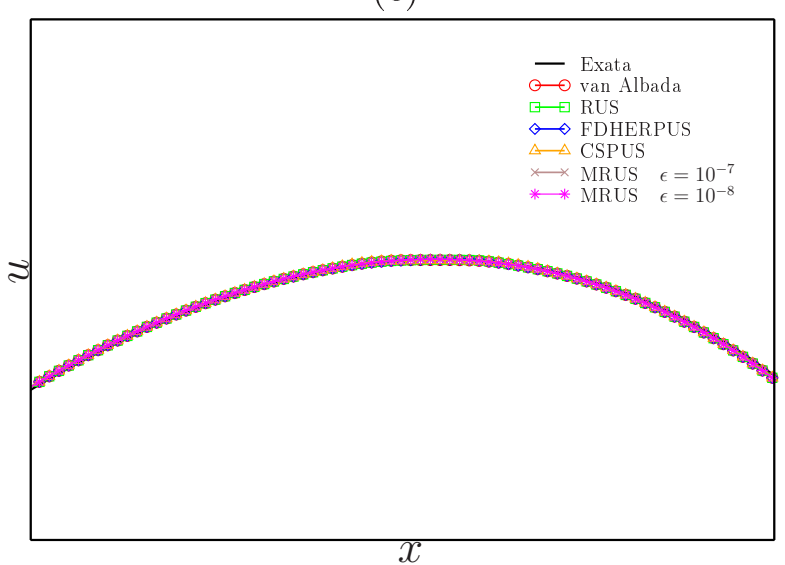

Figura 6.6: (a) Soluções exata e numéricas para equação não-linear de Burgers antes e após do choque. As aproximações são obtidas pelos esquemas: van Albada, RUS, FDHERPUS, CSPUS, MRUS para $\epsilon=10^{-7}$ e $\epsilon=10^{-8}$. Detalhes são mostrados em (b), (c), (d) e (e). 
Tabela 6.3: Erro relativo $E_{h}$ e estimativa para a ordem de convergência $q$.

\begin{tabular}{|c|c|c|c|c|c|}
\hline \multirow[t]{2}{*}{ Esquema } & \multirow[t]{2}{*}{ Malha } & \multicolumn{2}{|c|}{ Antes do choque } & \multicolumn{2}{|c|}{ Depois do choque } \\
\hline & & Erro & Ordem & Erro & Ordem \\
\hline \multicolumn{6}{|l|}{ van } \\
\hline \multirow[t]{6}{*}{ Albada } & 20 & 0.03180 & - & 0.023507 & - \\
\hline & 40 & 0.00799 & 2.000000 & 0.012033 & 0.498711 \\
\hline & 80 & 0.00198 & 1.992759 & 0.005989 & 0.483071 \\
\hline & 160 & 0.00049 & 2.012695 & 0.003023 & 0.503266 \\
\hline & 320 & 0.00012 & 2.014647 & 0.001514 & 0.493214 \\
\hline & 640 & 0.00003 & 2.029747 & 0.000758 & 0.498903 \\
\hline \multirow[t]{6}{*}{ FDHERPUS } & 20 & 0.03006 & - & 0.022010 & - \\
\hline & 40 & 0.00767 & 2.000000 & 0.011308 & 0.995987 \\
\hline & 80 & 0.00193 & 1.970547 & 0.005678 & 0.960815 \\
\hline & 160 & 0.00048 & 1.990626 & 0.002870 & 0.993889 \\
\hline & 320 & 0.00012 & 2.007495 & 0.001436 & 0.984332 \\
\hline & 640 & 0.00003 & 2.000000 & 0.000720 & 0.998995 \\
\hline \multirow[t]{6}{*}{ CSPUS } & 20 & 0.03073 & - & 0.021063 & - \\
\hline & 40 & 0.00783 & 2.000000 & 0.010735 & 0.997839 \\
\hline & 80 & 0.00195 & 1.972564 & 0.005319 & 0.972389 \\
\hline & 160 & 0.00049 & 2.005538 & 0.002673 & 1.013095 \\
\hline & 320 & 0.00012 & 1.992620 & 0.001334 & 0.992695 \\
\hline & 640 & 0.00003 & 2.029747 & 0.000668 & 1.002701 \\
\hline \multirow[t]{6}{*}{ RUS } & 20 & 0.03175 & - & 0.023636 & - \\
\hline & 40 & 0.00798 & 2.000000 & 0.012034 & 0.999044 \\
\hline & 80 & 0.00198 & 1.992296 & 0.005989 & 0.973870 \\
\hline & 160 & 0.00049 & 2.010888 & 0.003015 & 1.006729 \\
\hline & 320 & 0.00012 & 2.014647 & 0.001509 & 0.990157 \\
\hline & 640 & 0.00003 & 2.029747 & 0.000755 & 0.998565 \\
\hline \multirow[t]{6}{*}{$\begin{array}{l}\text { MRUS } \\
\epsilon=10^{-7}\end{array}$} & 20 & 0.03135 & - & 0.023091 & 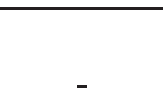 \\
\hline & 40 & 0.00792 & 2.000000 & 0.011718 & 0.992326 \\
\hline & 80 & 0.00196 & 1.984893 & 0.005921 & 0.980690 \\
\hline & 160 & 0.00049 & 2.014647 & 0.002984 & 0.979034 \\
\hline & 320 & 0.00012 & 2.000000 & 0.001502 & 0.978242 \\
\hline & 640 & 0.00003 & 2.029747 & 0.000755 & 0.986957 \\
\hline \multirow[t]{6}{*}{$\begin{array}{l}\text { MRUS } \\
\epsilon=10^{-8}\end{array}$} & 20 & 0.03146 & - & 0.023091 & - \\
\hline & 40 & 0.00794 & 2.000000 & 0.011718 & 0.992336 \\
\hline & 80 & 0.00197 & 1.986308 & 0.005921 & 0.978604 \\
\hline & 160 & 0.00049 & 2.010943 & 0.002984 & 0.984814 \\
\hline & 320 & 0.00012 & 2.007342 & 0.001502 & 0.988593 \\
\hline & 640 & 0.00003 & 2.029747 & 0.000755 & 0.990363 \\
\hline
\end{tabular}




\section{Teste 4: Caso viscoso}

Neste caso, a constante de viscosidade empregada foi $\nu=0.05$. A condição inicial utilizada foi à sugerida por Leveque (2004) [41]

$$
u(x, 0)=\left\{\begin{array}{lc}
1, & x<-80 \nu, \\
\frac{1}{2}(1-\tanh (x)), & -80 \nu \leq x \leq 80 \nu, \\
0, & 80 \nu<x,
\end{array}\right.
$$

A solução exata do problema é dada por

$$
u(x)=1-\frac{1}{2}\left(1-\tanh \left(-\frac{x-0.5 t}{4 \nu}\right)\right), \quad x \in[-2,3] .
$$

Para resolver este problema foram utilizadas 150 células computacionais, número de Courant $C=0.9$ e tempo final de simulação $t=1.0$. Os resultados numéricos comparados à solução analítica (6.6) estão mostrados na Figura (6.7), onde pode-se perceber (globalmente) que os resultados com os esquemas van Albada, RUS, FDHERPUS, CSPUS e MRUS são próximos à solução exata; na região Zoom 1 ambos os esquemas FDHERPUS e MRUS forneceram os melhores resultados, ao passo que na região Zoom 2 van Albada e CSPUS foram os melhores esquemas.

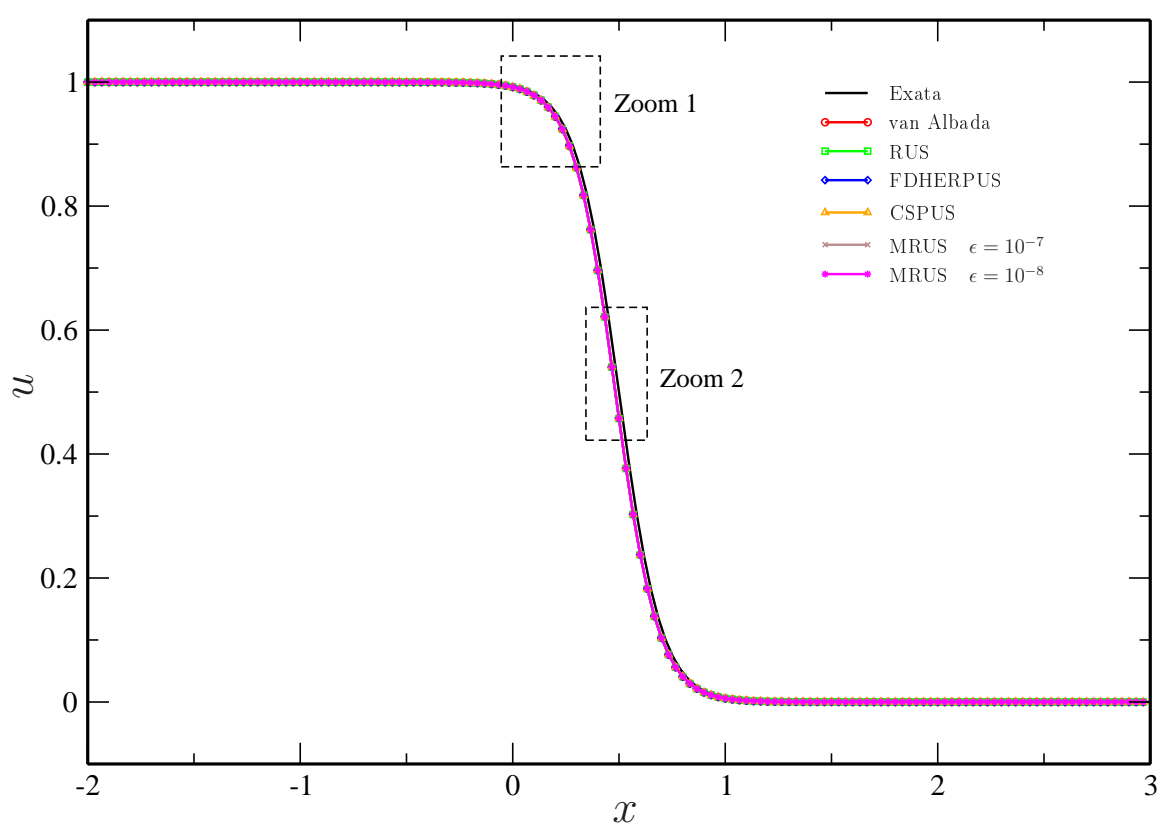



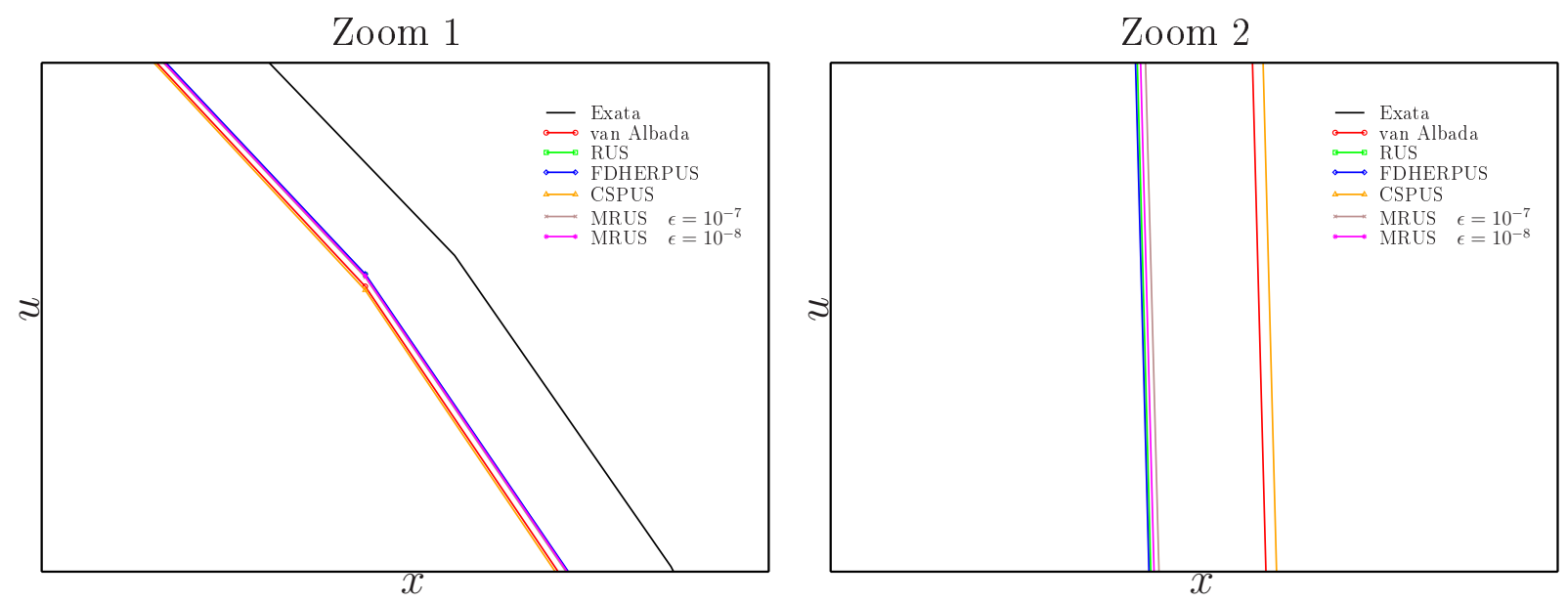

Figura 6.7: Detalhes da solução exata e dos resultados computacionais para equação não-linear de Burgers no caso viscoso mostrando o aumento de algumas regiões. As aproximações são obtidas pelos esquemas: van Albada, RUS, FDHERPUS, CSPUS, MRUS para $\epsilon=10^{-7} \mathrm{e}$ $\epsilon=10^{-8}$.

Foi realizada também para este problema análise TVD com o uso dos esquema van Albada, RUS, FDHERPUS e CSPUS. Estas informações estão mostradas na Figura 6.8, em que se observa não crescimento dessas TVs conforme o tempo avança, confirmando portanto estabilidade.

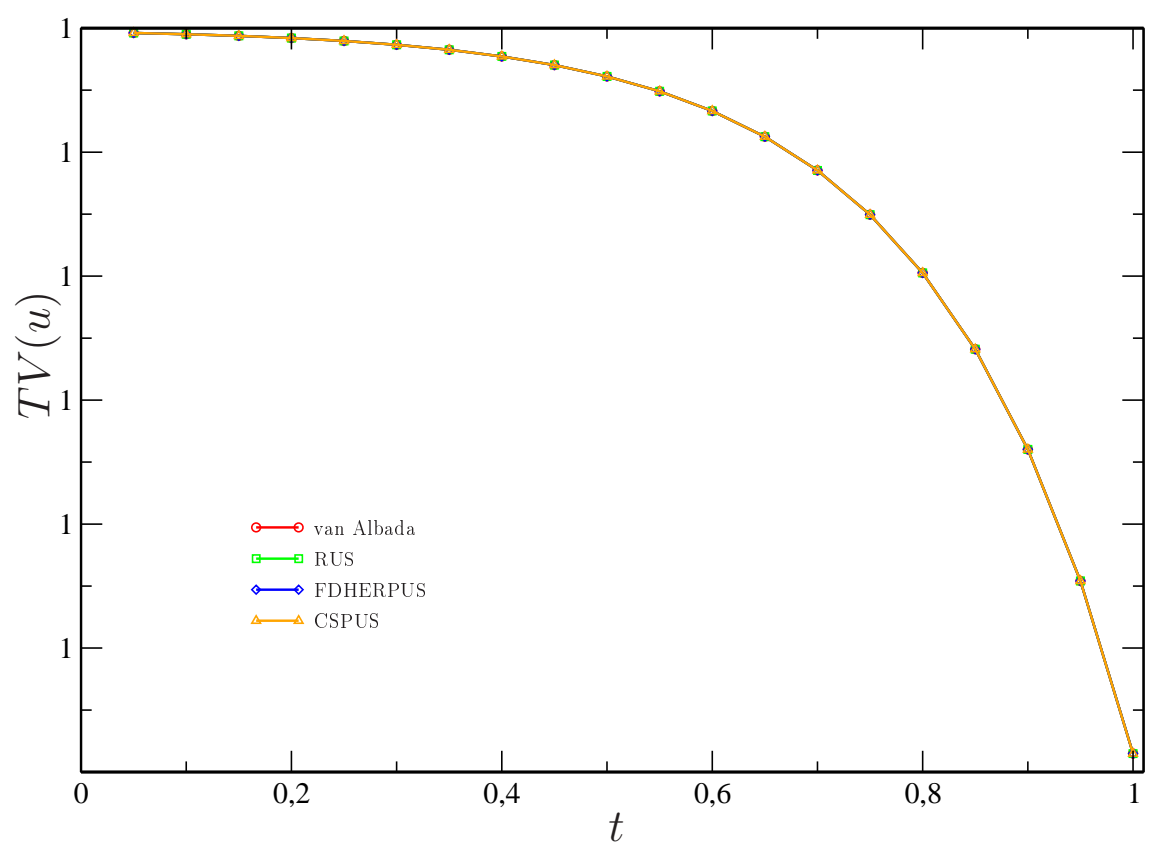

Figura 6.8: Análise da TV feita para o Teste 4, mostrando o comportamento dos esquemas. 


\subsection{Equações de Euler}

Nesta seção são apresentados dois testes realizados com as equações de Euler unidimensional (2.9): o primeiro refere-se ao problema tubo de choque de Shu-Osher (ver, por exemplo, Osher (1989) [63]); e o segundo teste constitui o problema de fortes interações de choques (ver, por exemplo, Collela (1984) [83]). Ambos os testes são de grande interesse, neste trabalho, por modelarem bem vários problemas complexos em fluido dinâmica computacional.

\section{Teste 5: Tubo de choque de Shu e Osher}

Este problema simula uma onda acústica com uma perturbação senoidal na variável densidade $\rho$. É um caso interessante pois envolve tanto estruturas oscilatórias suaves como também choques. As equações de Euler 1D são resolvidas considerando as condições iniciais

$$
\left(\rho_{0}, u_{0}, p_{0}\right)^{T}= \begin{cases}(3.857143,2.629369,10.333333)^{T}, & x<-4 \\ (1+0.2 \sin (5 x), 0,1)^{T}, & x \geq-4 .\end{cases}
$$

Nas Figuras 6.9 - 6.14 são mostrados os resultados numéricos obtidos com os esquemas van Albada, RUS, FDHERPUS, CSPUS e MRUS com parâmetros $\epsilon=10^{-1}$ e $\epsilon=10^{-7}$, mais a solução de referência numa malha de 2000 células computacionais e a Courant $C=0.9$; esta solução está determinada com o método de Godunov com termo de correção e Superbee como limitador de fluxo (ver Leveque (2004) [41]).

As simulações foram realizadas em diferentes malhas (de 200 até 800 células) e a vários números de Courant (de 0.1 até 0.9). O propósito aqui foi o de comparar os desempenhos dos esquemas van Albada, RUS, FDHERPUS, CSPUS e MRUS com parâmetros $\epsilon=10^{-1} \mathrm{e}$ $\epsilon=10^{-7}$ em diferentes resoluções da malha e condições de estabilidade. Pode-se notar por essas Figuras que ao se refinar a malha e, ao mesmo tempo, variar o número de Courant as soluções ficaram cada vez mais próximas da solução de referência, sendo que os melhores resultados foram derivados com o uso do esquema MRUS com parâmetro $\epsilon=10^{-1}$. Nota-se ainda que os resultado com o esquema van Albada ficou em quarto lugar na classificação geral, e o esquema CSPUS forneceu os piores resultados.

\section{Teste 6: Fortes interações de choques}

O problema de fortes interações de choques consiste em resolver o sistema hiperbólico não 
(a)

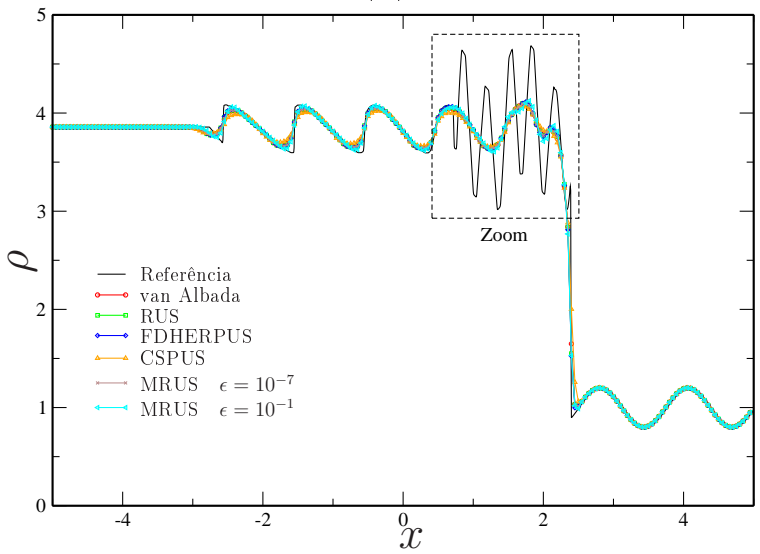

(b)

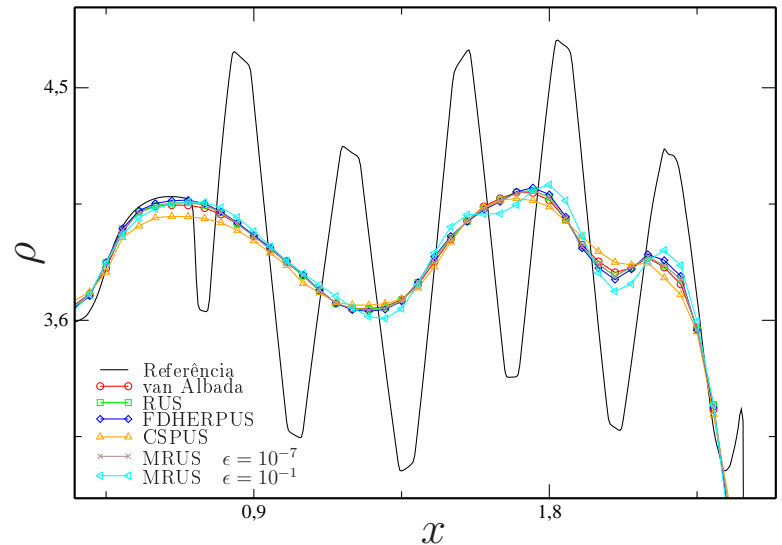

Figura 6.9: (a) Solução da equação de Euler 1D para o teste de Shu-Osher com $N=200$ e $C=0.5$. (b) Região de ampliação.

(a)

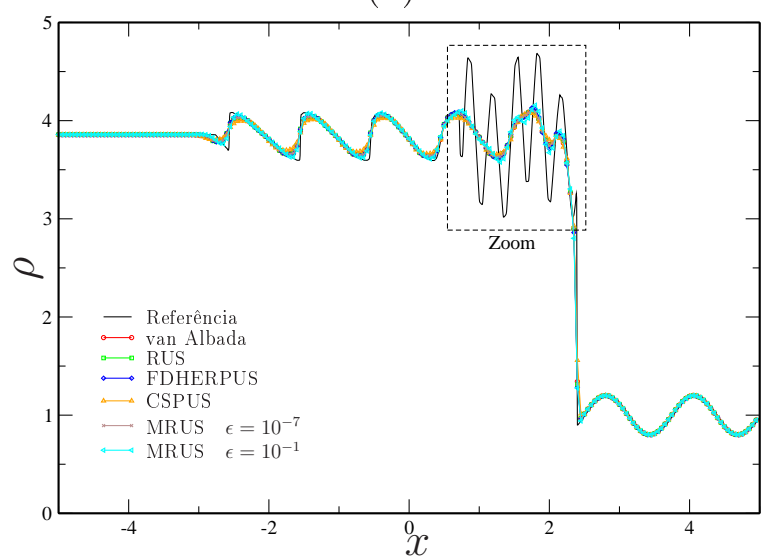

(b)

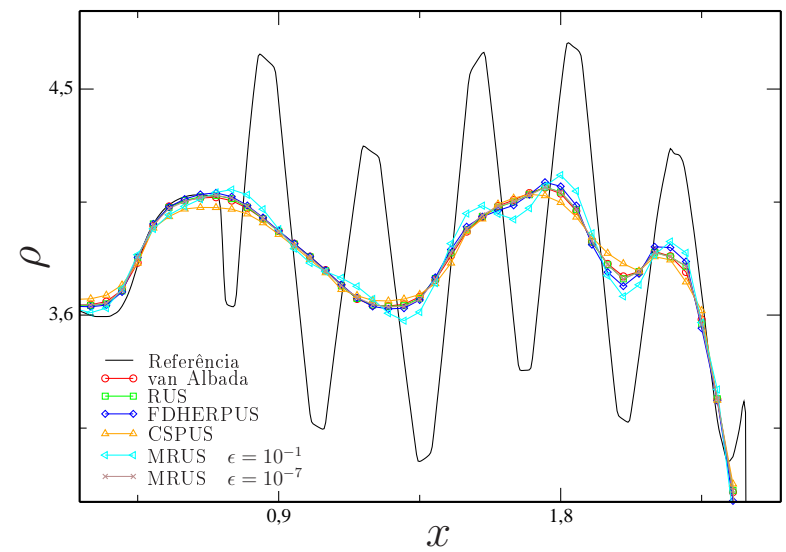

Figura 6.10: (a) Solução da equação de Euler 1D para o teste de Shu-Osher com $N=200$ e $C=0.9$. (b) Região de ampliação.

(a)

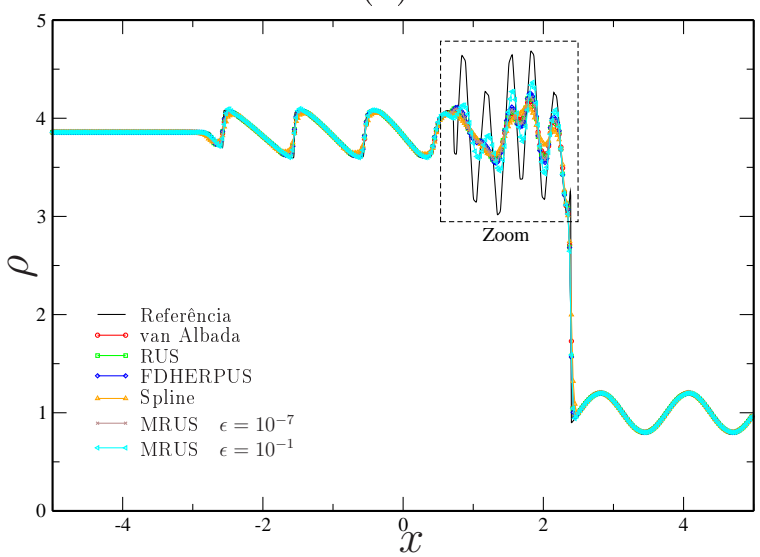

(b)

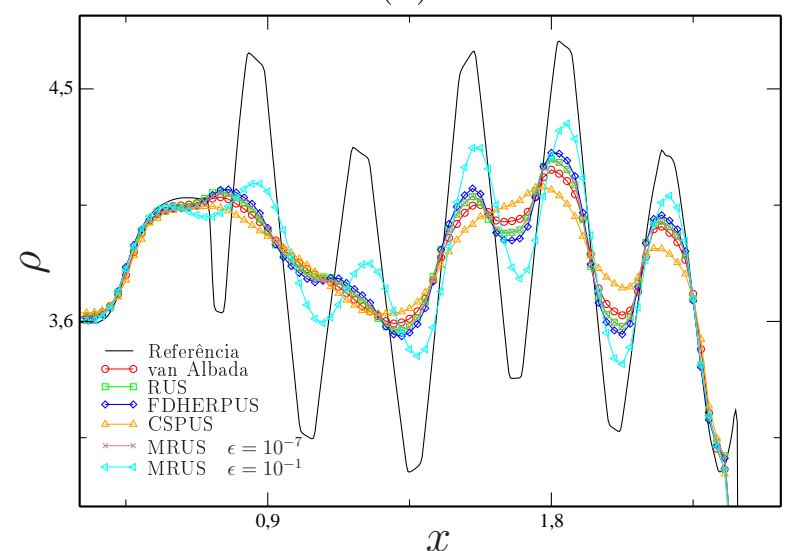

Figura 6.11: (a) Solução da equação de Euler 1D para o teste de Shu-Osher com $N=400 \mathrm{e}$ $C=0.1$. (b) Região de ampliação. 
(a)

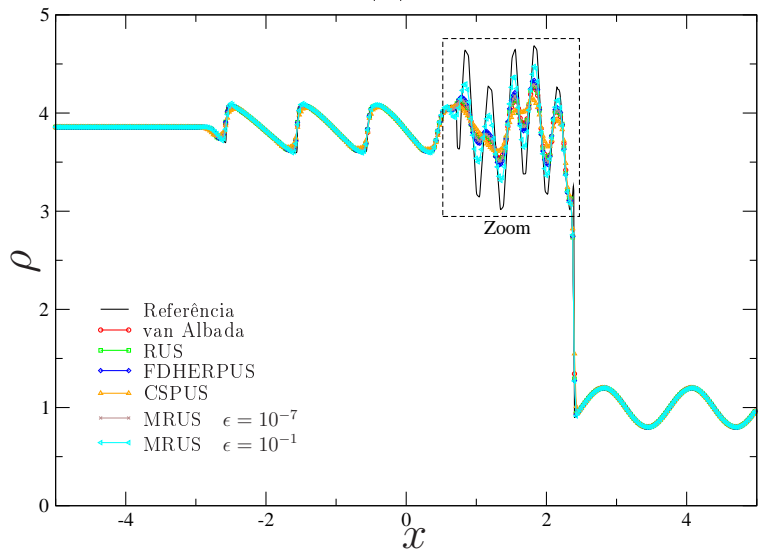

(b)

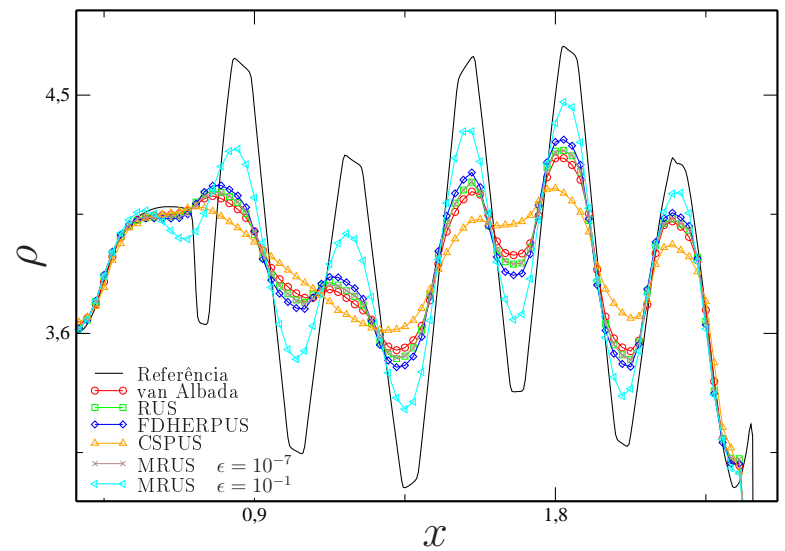

Figura 6.12: (a) Solução da equação de Euler 1D para o teste de Shu-Osher com $N=400$ e $C=0.8$. (b) Região de ampliação.

(a)

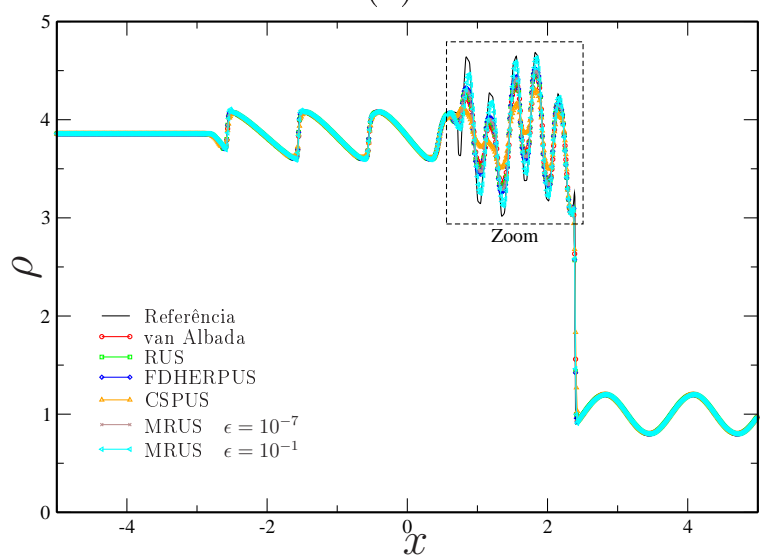

(b)

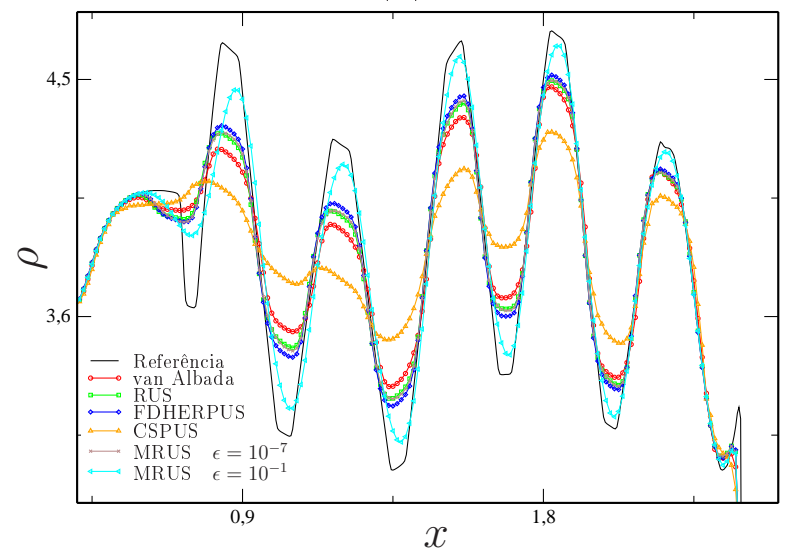

Figura 6.13: (a) Solução da equação de Euler 1D para o teste de Shu-Osher com $N=800$ e $C=0.1$. (b) Região de ampliação.

(a)

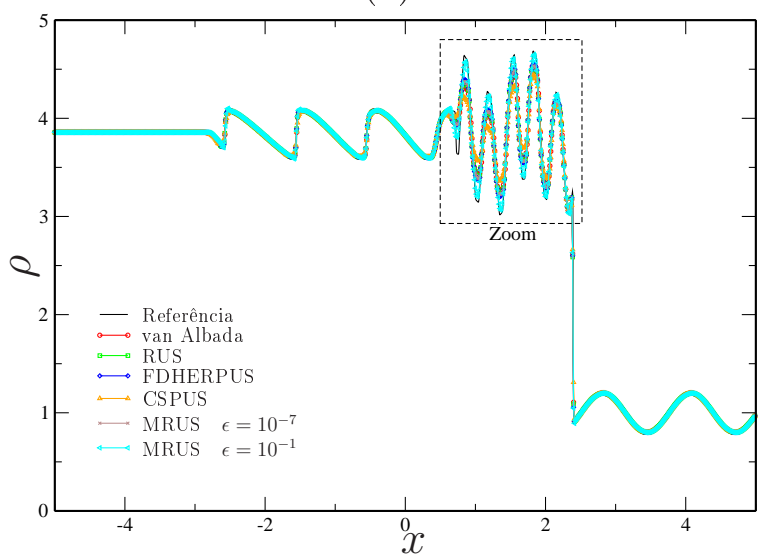

(b)

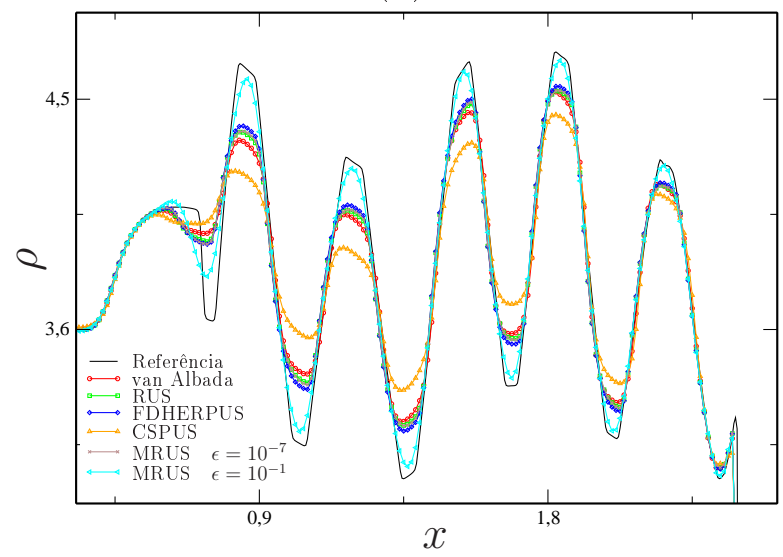

Figura 6.14: (a) Solução da equação de Euler 1D para o teste de Shu-Osher com $N=800 \mathrm{e}$ $C=0.5$. (b) Região de ampliação. 
linear de Euler 1D sujeito a três estados iniciais constantes

$$
\left(\rho_{0}, u_{0}, p_{0}\right)^{T}=\left\{\begin{array}{cc}
\left(1,0,10^{3}\right)^{T}, & 0 \leq x<0.1 \\
\left(1,0,10^{-2}\right)^{T}, & 0.1 \leq x<0.9 \\
\left(1,0,10^{2}\right)^{T}, & 0.9 \leq x \leq 1.0 .
\end{array}\right.
$$

Fisicamente, estas condições iniciais dão origem à descontinuidades de contato e (fortes) ondas de choques as quais são transportadas domínio adentro; estes choques colidem entre si e ondas de choques se refletem na direção do contorno. O desempenho dos esquemas van Albada, RUS, FDHERPUS, CSPUS e MRUS, com parâmetros $\epsilon=10^{-7}$ e $\epsilon=10^{-8}$ foi investigado na solução deste problema complexo. Para tanto, considerou-se como solução de referência à obtida com o método de Godunov com termo de correção e o limitador de fluxo MC (van Leer (1977) [78]); a simulação (de referência) foi realizada numa malha com 2000 células computacionais, a número de Courant $C=0.9$ e tempo final de simulação $t=0.038$.

Os resultados numéricos, usando-se uma malha com $N=1000$ células computacionais e número de Courant $C=0.9$, para a densidade $\rho$, a energia $E$ e a velocidade $u$ podem ser vistos nas Figuras 6.15, 6.16 e 6.17, respectivamente. Por essas Figuras, vê-se que em escala global todos os esquemas forneceram resultados semelhentes, com o esquema FDHERPUS fornecendo uma solução numérica (ligeiramente) melhor em algumas regiões; e o esquema van Albada (consagrado em escoamentos de fluidos compressíveis) ficou em quarto lugar na classificação geral. Estas informações são também confirmadas na Tabela 6.4, onde constam os erros relativos e as ordens de convergência observadas. 


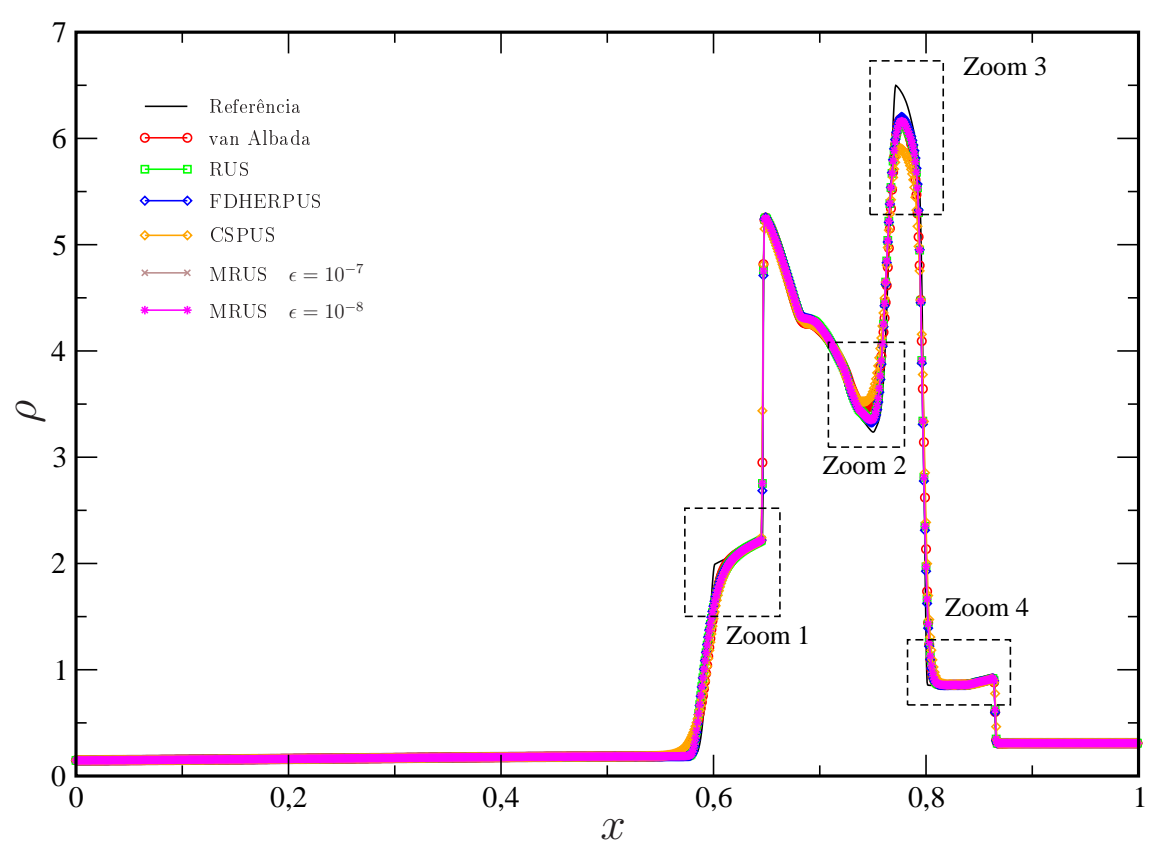

Zoom 1

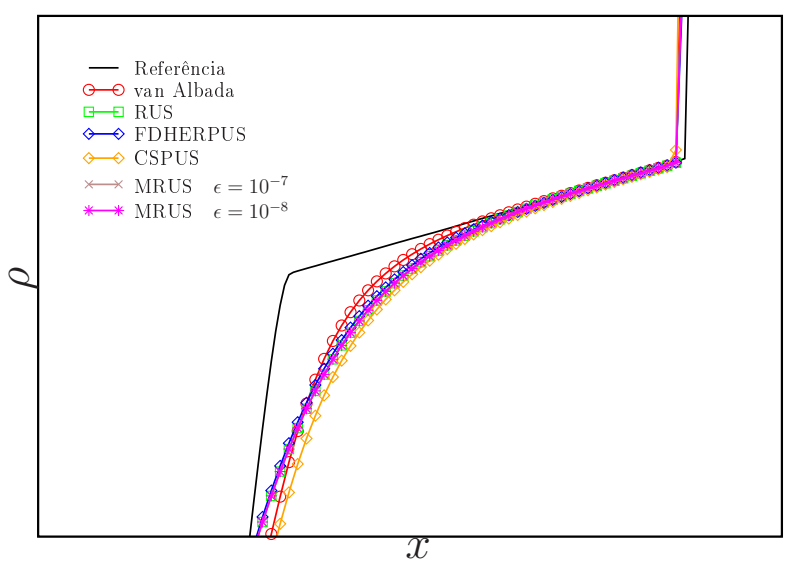

Zoom 2

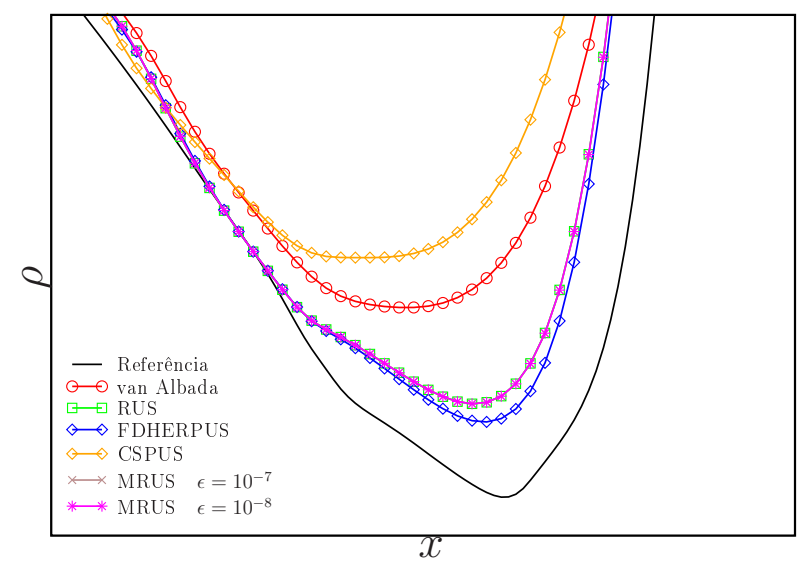

Zoom 3

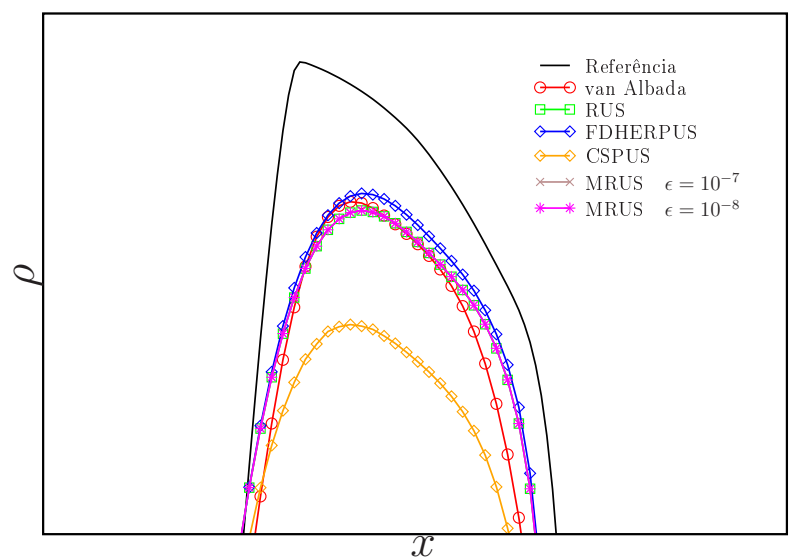

Zoom 4

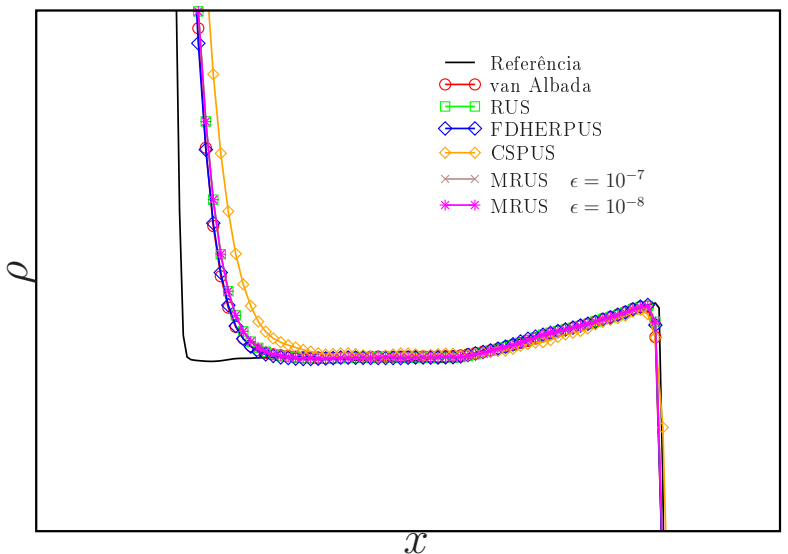

Figura 6.15: Soluções de referência e numéricas (globais e detalhes) para a densidade $\rho$ no problema envolvendo fortes interações de choques. 


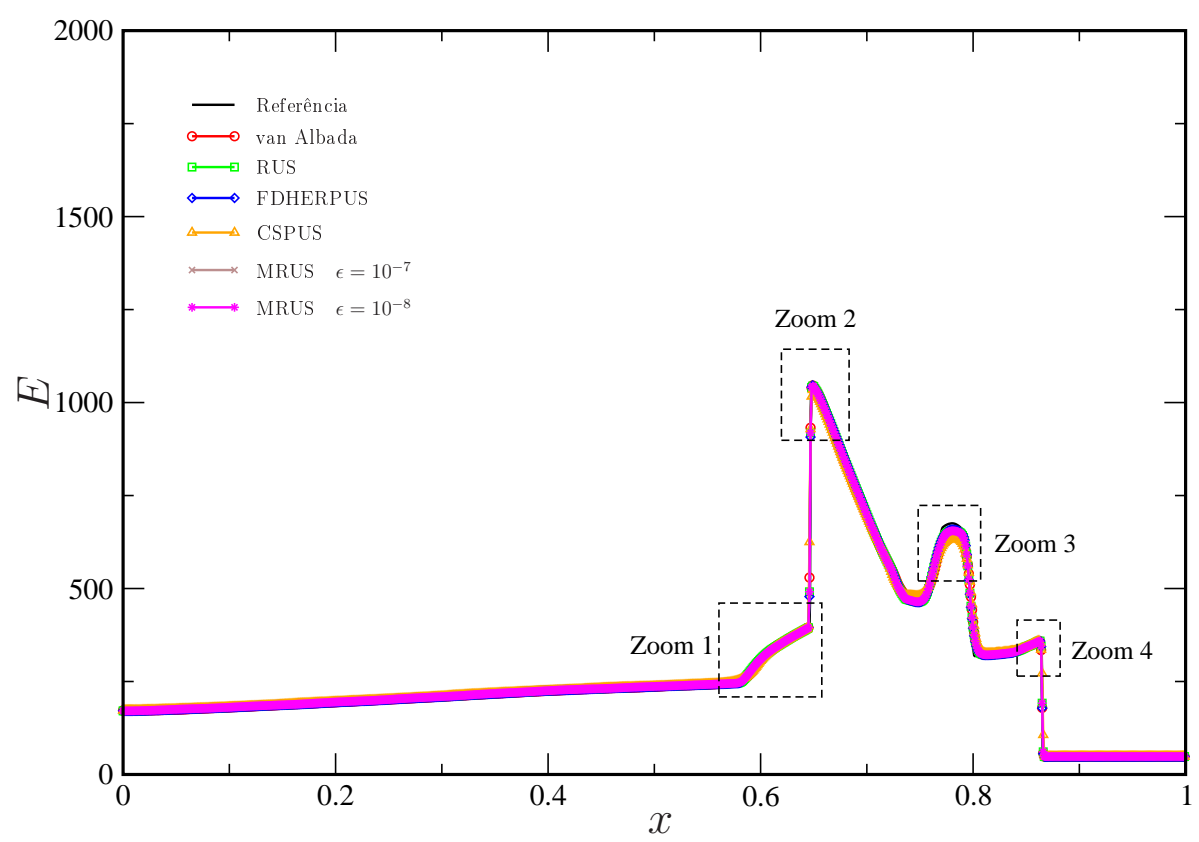

Zoom 1

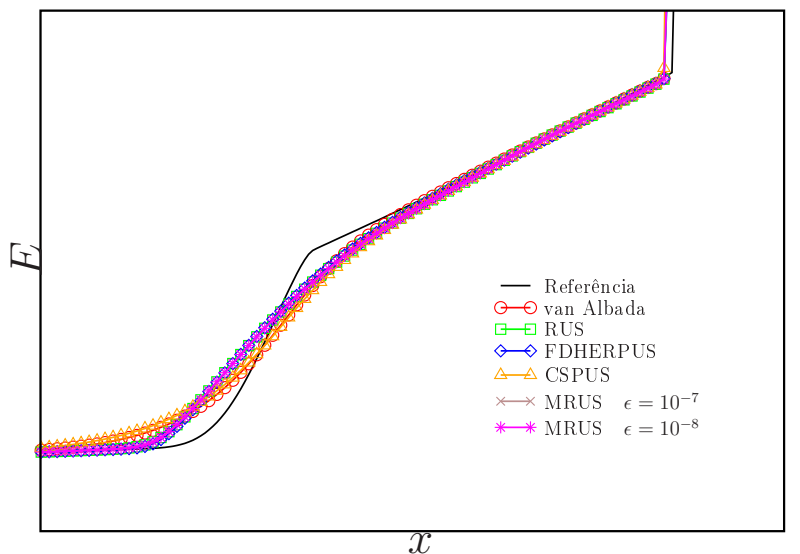

Zoom 3

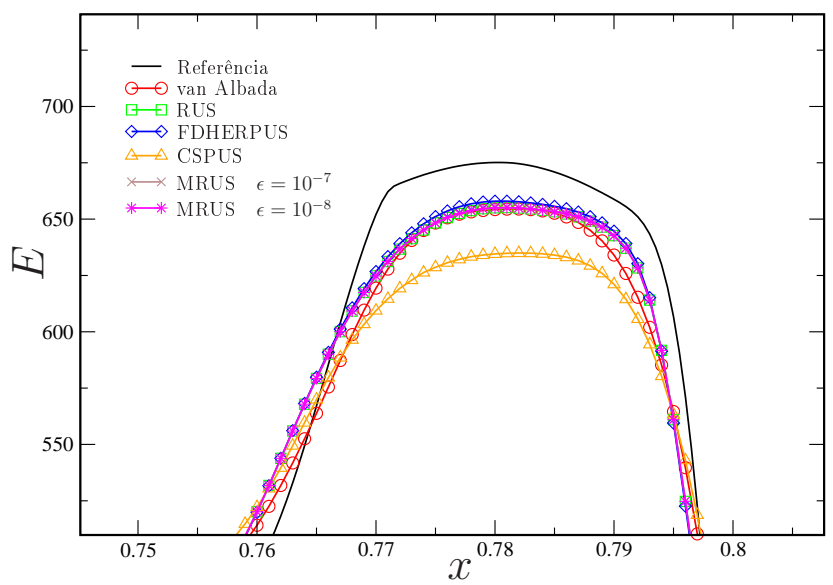

Zoom 2

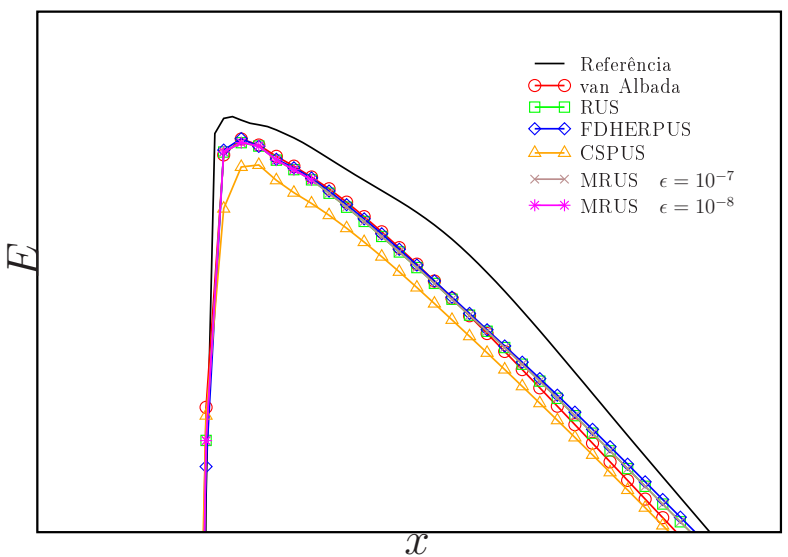

Zoom 4

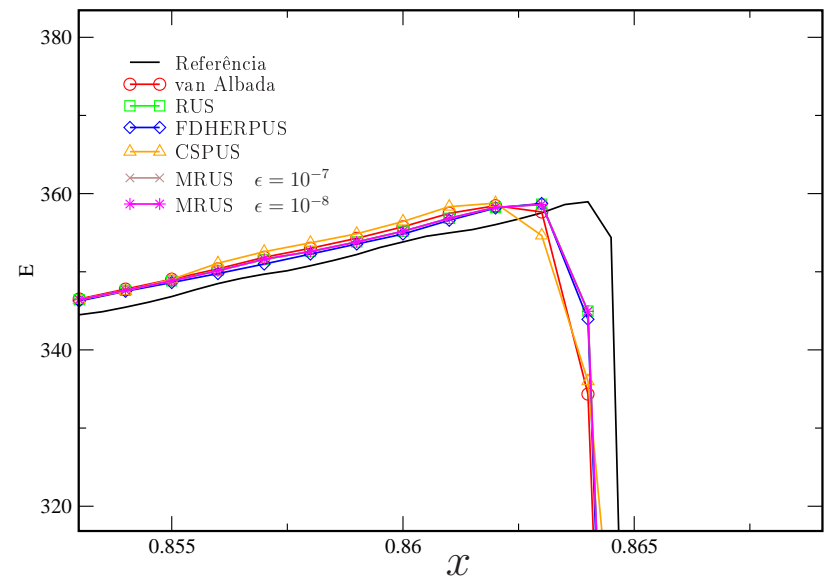

Figura 6.16: Soluções de referência e numéricas (globais e detalhes) para a energia $E$ no problema envolvendo fortes interações de choques. 


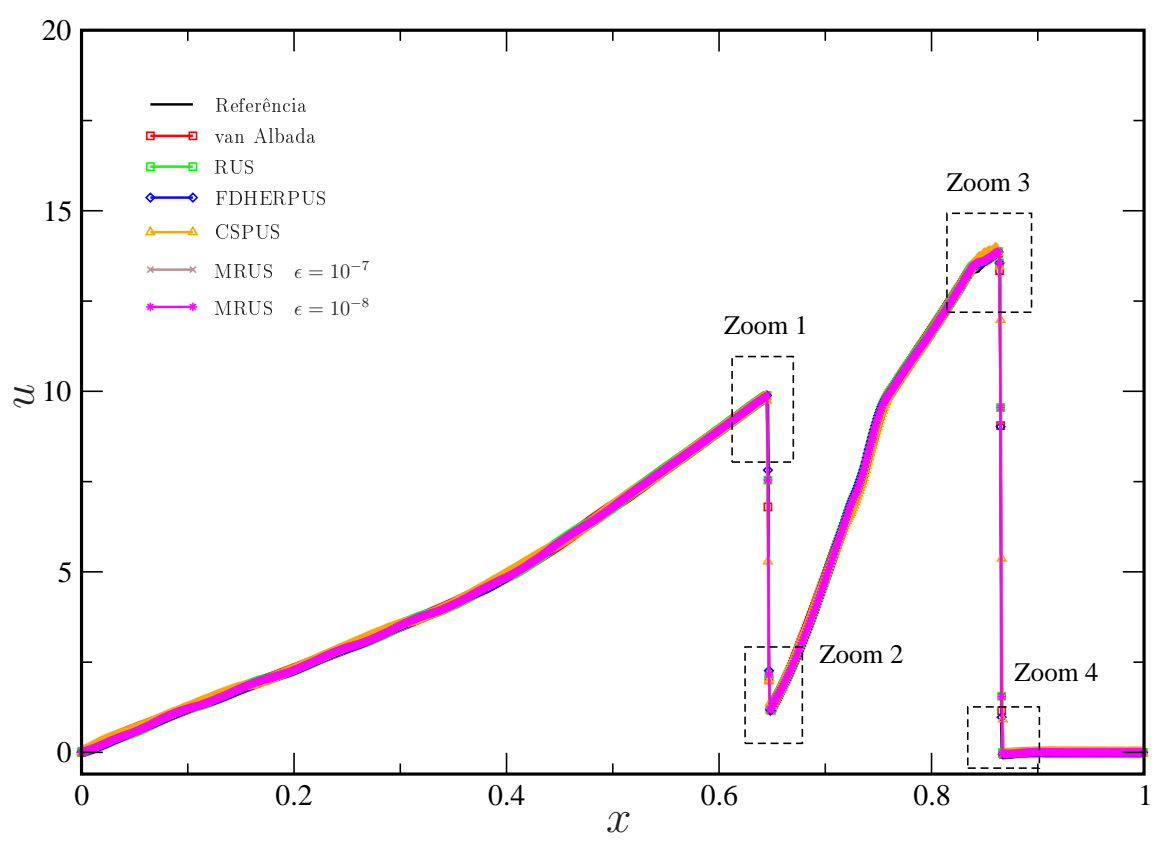

Zoom 1

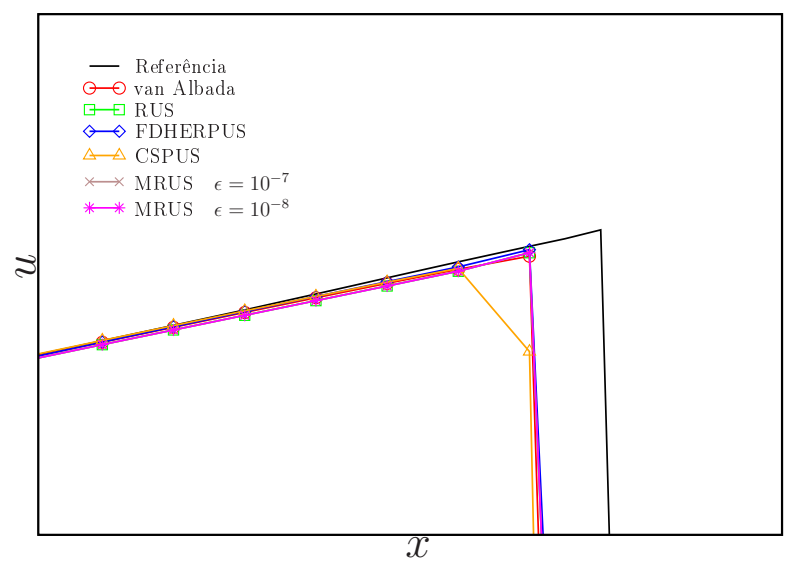

Zoom 3

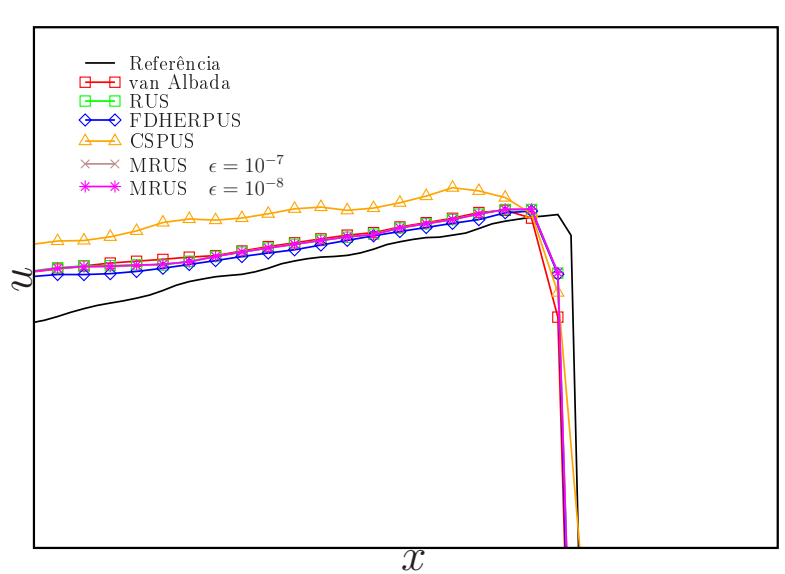

Zoom 2

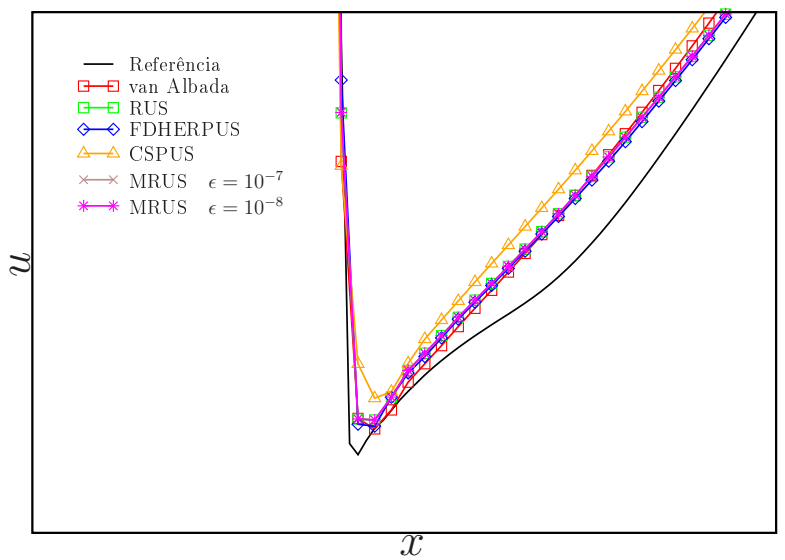

Zoom 4

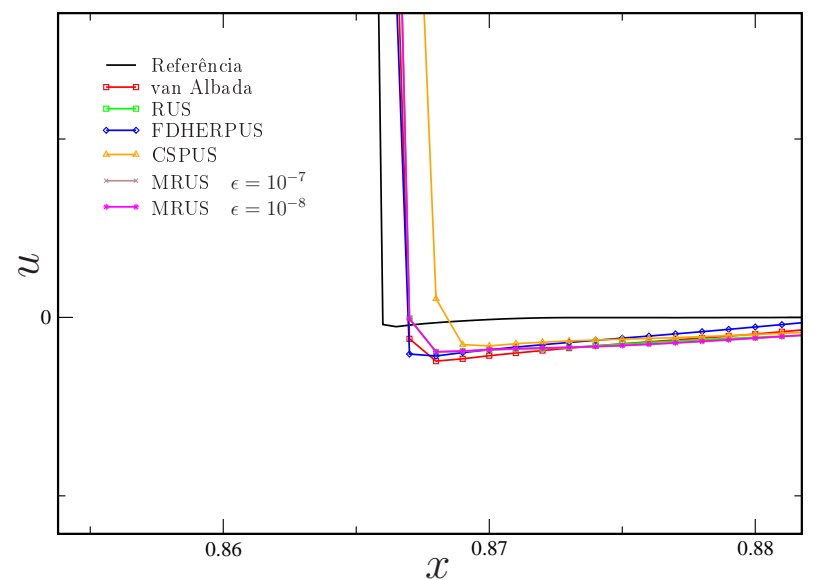

Figura 6.17: Soluções de referência e numéricas (globais e detalhes) para a velocidade $u$ no problema envolvendo fortes interações de choques. 
Tabela 6.4: Erro relativo $E_{h}$ e estimativa para a ordem de convergência $q$.

\begin{tabular}{|c|c|c|c|c|c|}
\hline \multirow[t]{2}{*}{ Esquema } & \multirow[t]{2}{*}{ Malha } & \multicolumn{2}{|c|}{$L_{1}$} & \multicolumn{2}{|c|}{$L_{2}$} \\
\hline & & Erro & Ordem & Erro & Ordem \\
\hline \multicolumn{6}{|l|}{ van } \\
\hline \multirow[t]{4}{*}{ Albada } & 125 & 0.249525 & - & 0.308433 & - \\
\hline & 250 & 0.153647 & 0.6995 & 0.213306 & 0.5320 \\
\hline & 500 & 0.082280 & 0.9010 & 0.125368 & 0.7667 \\
\hline & 1000 & 0.036122 & 1.1876 & 0.070791 & 0.8245 \\
\hline \multirow[t]{4}{*}{ RUS } & 125 & 0.237404 & - & 0.301842 & - \\
\hline & 250 & 0.143472 & 1.0512 & 0.200937 & 0.7545 \\
\hline & 500 & 0.074552 & 0.7070 & 0.118827 & 0.5870 \\
\hline & 1000 & 0.032366 & 0.9093 & 0.070433 & 0.7578 \\
\hline \multirow[t]{4}{*}{ FDHERPUS } & 125 & 0.232792 & - & 0.300370 & - \\
\hline & 250 & 0.137936 & 1.0613 & 0.195192 & 0.7383 \\
\hline & 500 & 0.070587 & 0.7352 & 0.113810 & 0.6218 \\
\hline & 1000 & 0.030148 & 0.9298 & 0.068219 & 0.7782 \\
\hline \multirow[t]{4}{*}{ CSPUS } & 125 & 0.278921 & - & 0.324136 & - \\
\hline & 250 & 0.177699 & 1.0031 & 0.238374 & 0.8512 \\
\hline & 500 & 0.107647 & 0.6323 & 0.161276 & 0.4433 \\
\hline & 1000 & 0.050713 & 0.7320 & 0.089399 & 0.5636 \\
\hline \multirow{5}{*}{$\begin{array}{l}\text { MRUS } \\
\epsilon=10^{-7}\end{array}$} & & & & & \\
\hline & 125 & 0.237404 & - & 0.301848 & - \\
\hline & 250 & 0.143481 & 1.0516 & 0.200956 & 0.7547 \\
\hline & 500 & 0.074552 & 0.7069 & 0.118805 & 0.5869 \\
\hline & 1000 & 0.032364 & 0.9094 & 0.070412 & 0.7582 \\
\hline \multirow{5}{*}{$\begin{array}{l}\text { MRUS } \\
\epsilon=10^{-8}\end{array}$} & & & & & \\
\hline & 125 & 0.237398 & - & 0.301837 & - \\
\hline & 250 & 0.143478 & 1.0514 & 0.200951 & 0.7546 \\
\hline & 500 & 0.074551 & 0.7069 & 0.118815 & 0.5869 \\
\hline & 1000 & 0.032365 & 0.9094 & 0.070422 & 0.7581 \\
\hline
\end{tabular}




\subsection{Problemas modelados pelas equações de águas rasas}

Nesta seção são apresentados resultados numéricos derivados com o uso dos esquemas van Albada, FDHERPUS, RUS, CSPUS e MRUS para problemas modelados pelas equações de águas rasas (2.13). Em particlular, foram simulados dois problemas de interesse em fluido dinâmica computacional; sendo que o primeiro deles é o problema de uma onda de água conhecida como salto hidráulico e o segundo o problema da ruptura de uma barragem (ver por exemplo LeVeque (2004) [41]).

\section{Teste 7: Salto hidráulico}

O fenômeno salto hidráulico representa um problema não linear interessante para as leis de conservação hiperbólicas de águas rasas, e consiste na evolução de duas ondas de água (em sentidos opostos) após o rompimento de uma onda de água inicialmente em repouso. As condições iniciais consideradas são

$$
h_{0}(x)=\left\{\begin{array}{l}
\frac{1}{2}\left(1-x^{2}\right)+1,-1 \leq x \leq 1, \\
1, \text { em caso contrário. }
\end{array} \text { e } \quad u_{0}(x)=0 .\right.
$$

Os seguintes dados foram empregados nas simulações: uma malha com 500 células computacionais, número de Courant $C=0.9$ e tempos finais de simulação $t=0.0, t=1.0, t=2.0 \mathrm{e}$ $t=3.0$. Para a determinação da solução de referência, foi utilizado o método de Godunov com termo de correção e o limitador de fluxo MC numa malha de 2000 células computacionais e $C=0.9$. Na Figura 6.18 estão apresentados os resultados numéricos obtidos, onde os gráficos à esquerda correspondem a evolução da profundidade $h$ e os à direita correspondem à vazão hu. Pode-se concluir por essas Figuras que todos os esquemas implementados simulam bem o transporte de uma onda solitária via a equação de águas rasas. Em particular, no tempo $t=3$, o esquema MRUS com os parâmetros $\epsilon=10^{-7}$ e $\epsilon=10^{-8}$ simulou melhor as regiões de descontinuidade; e o esquema de van Albada foi classificado como um dos piores nessas regiões, ganhando apenas do esquema CSPUS.

\section{Teste 8: Ruptura de uma barragem}

Neste teste, o desempenho dos esquemas RUS, FDHERPUS, CSPUS e MRUS, com parâmetros $\epsilon=10^{-7}$ e $\epsilon=10^{-8}$, e van Albada é avaliado na ruptura de uma barragem inicialmente na configuração da Figura 6.19. Nesta configuração tomam-se as alturas iniciais dos reservatórios e a velocidade inicial como 

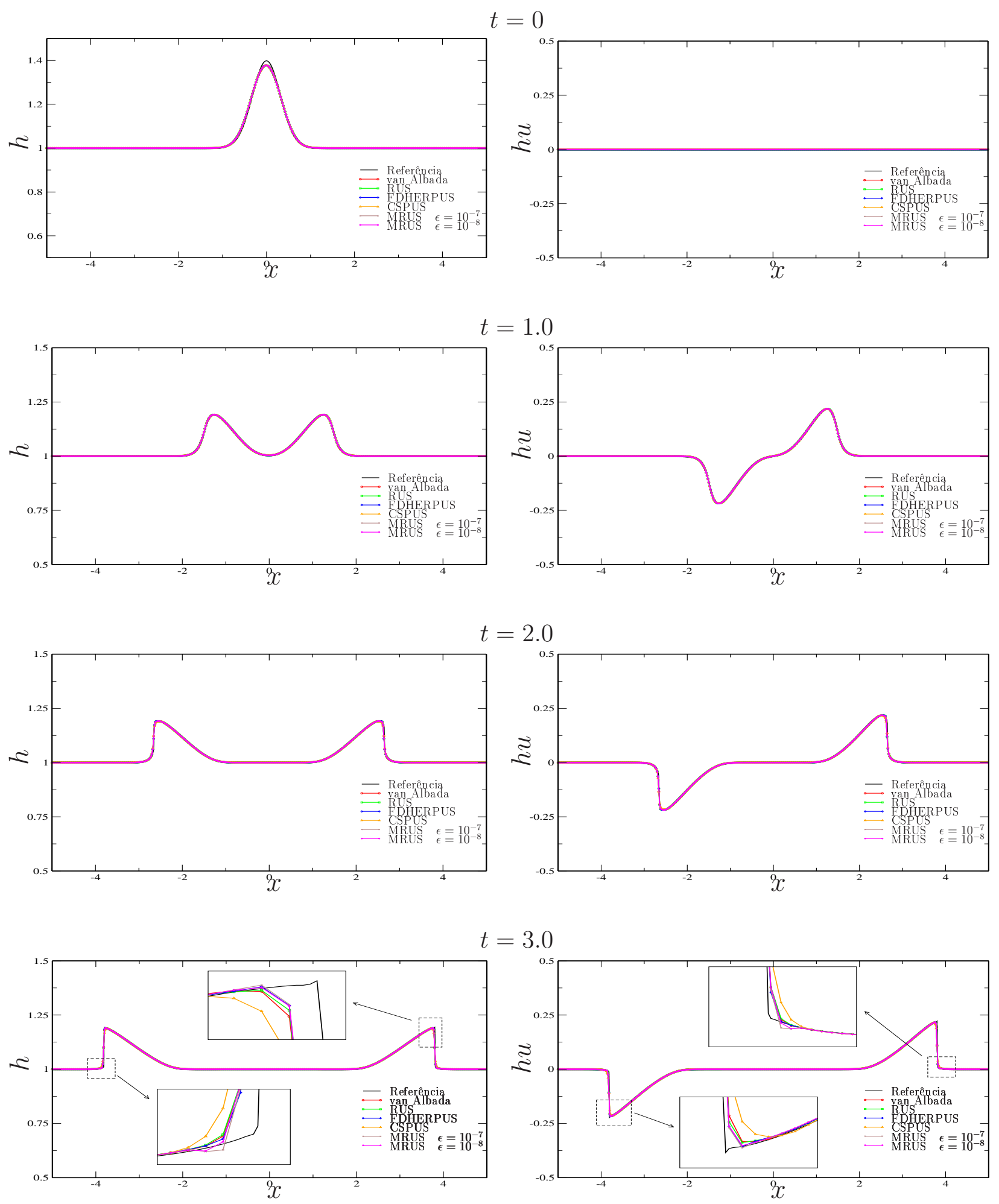

Figura 6.18: Evolução da profundidade $h$ (coluna à esquerda) e da vazão hu (coluna à direita). 


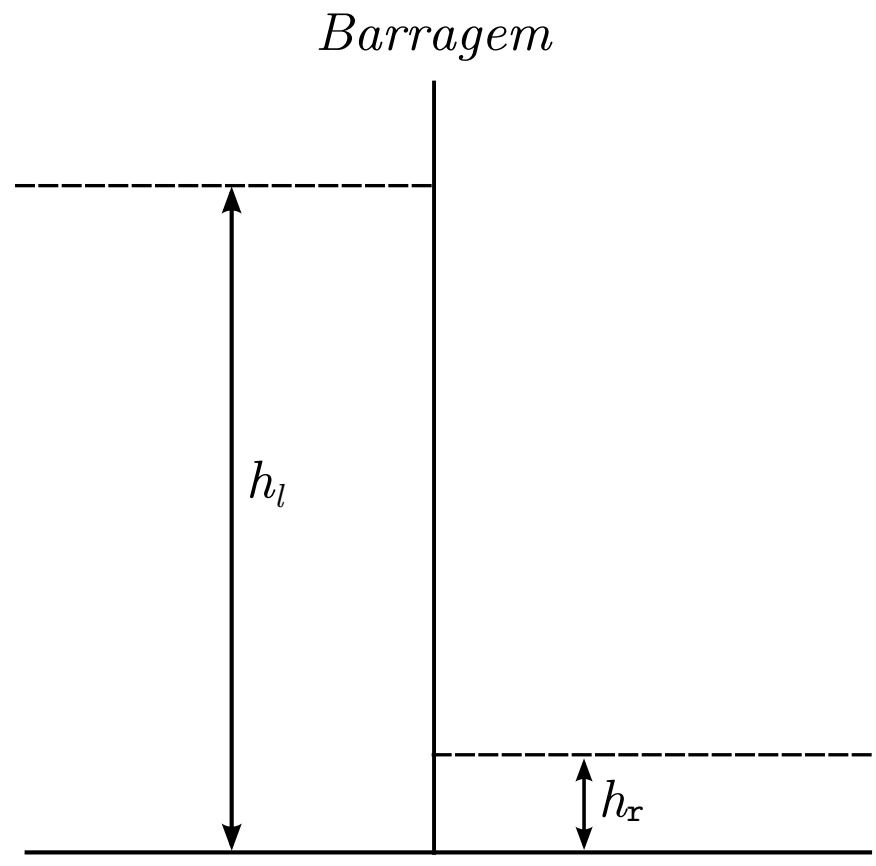

Figura 6.19: Problema Dam-break 1D

$$
h_{0}(x)=\left\{\begin{array}{ll}
h_{l}=3 & \text { se } \quad x \leq 0, \\
h_{r}=1 & \text { se } 0<x .
\end{array} \quad, u_{0}(x)=0 .\right.
$$

O problema a ser simulado aqui é aquele oriundo do rompimento instantâneo da barragem. Após o rompimento, cria-se uma onda de choque que se propaga no domínio. Os dados para simulação são: uma malha de $N=500$ células computacionais, número de Courant $C=0.7 \mathrm{e}$ tempo final $t=1.2$. Considerou-se como solução de referência o método de Godunov com termo de correção e o limitador de fluxo MC numa malha de 2000 células computacionais e $C=0.7$. Nas Figuras 6.20 e 6.21 está mostrado a evolução da altura $h$ e da vazão $h u$, até o tempo $t=2$, respectivamente. Pode-se verificar por essas Figuras que são extraídas informações análogas às dos testes anteriores. A Tabela 6.5 contém os erros na norma $L_{1}$ e a ordem de convergência observada, o qual quantifica os resultados apresentados nas Figuras 6.20 e 6.21. O cálculo das TVs para os esquemas van Albada, RUS, FDHERPUS e CSPUS estão apresentados na Figura 6.22 , mostrando que ao se refinar a malha as TVs decrescem com o tempo. 

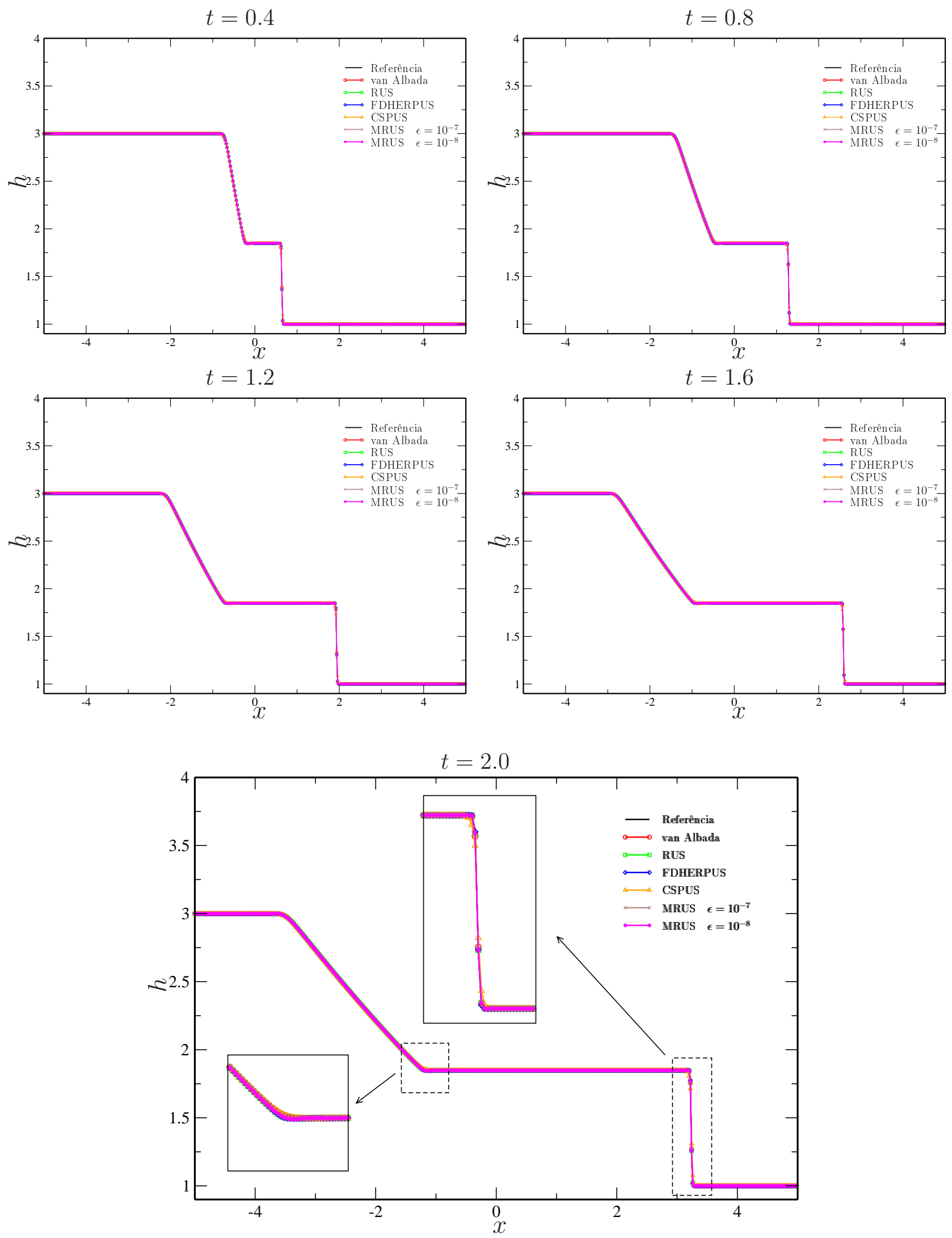

Figura 6.20: Evolução temporal para o problema de Dam-break. Ilustra-se as soluções exata e numérica na variável $h$. 

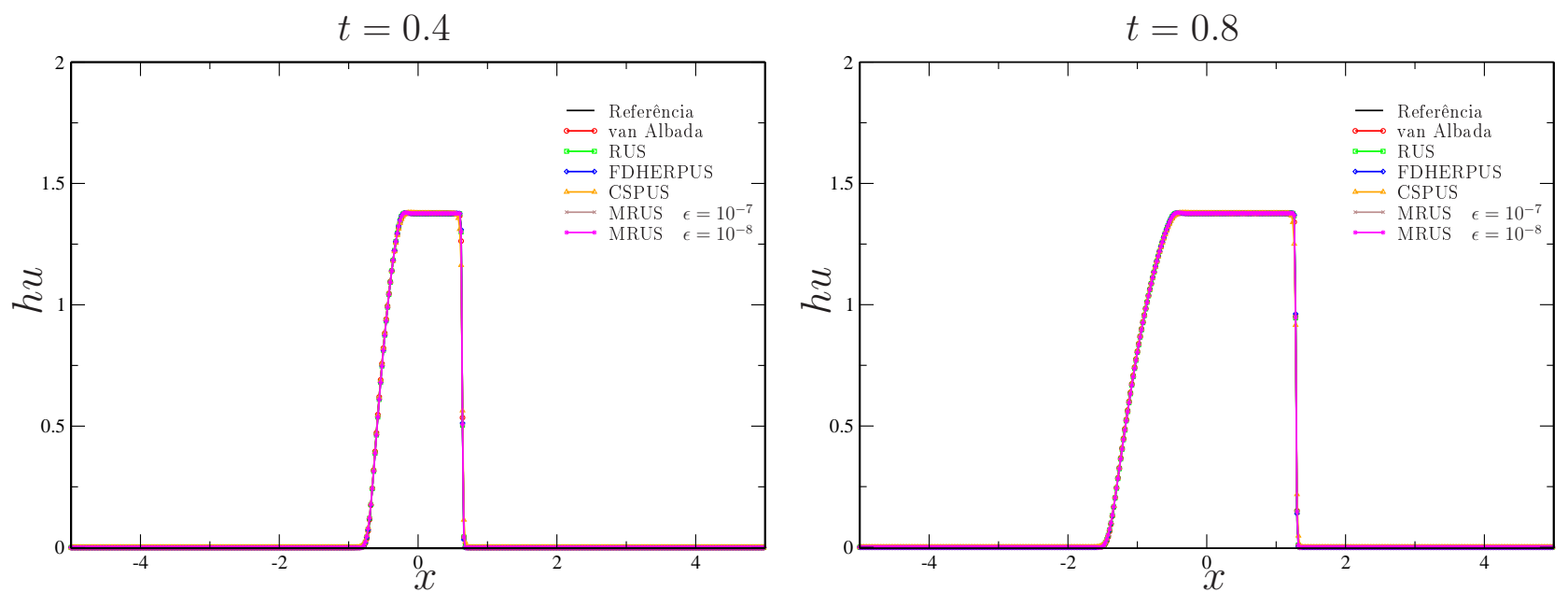

$t=1.2$
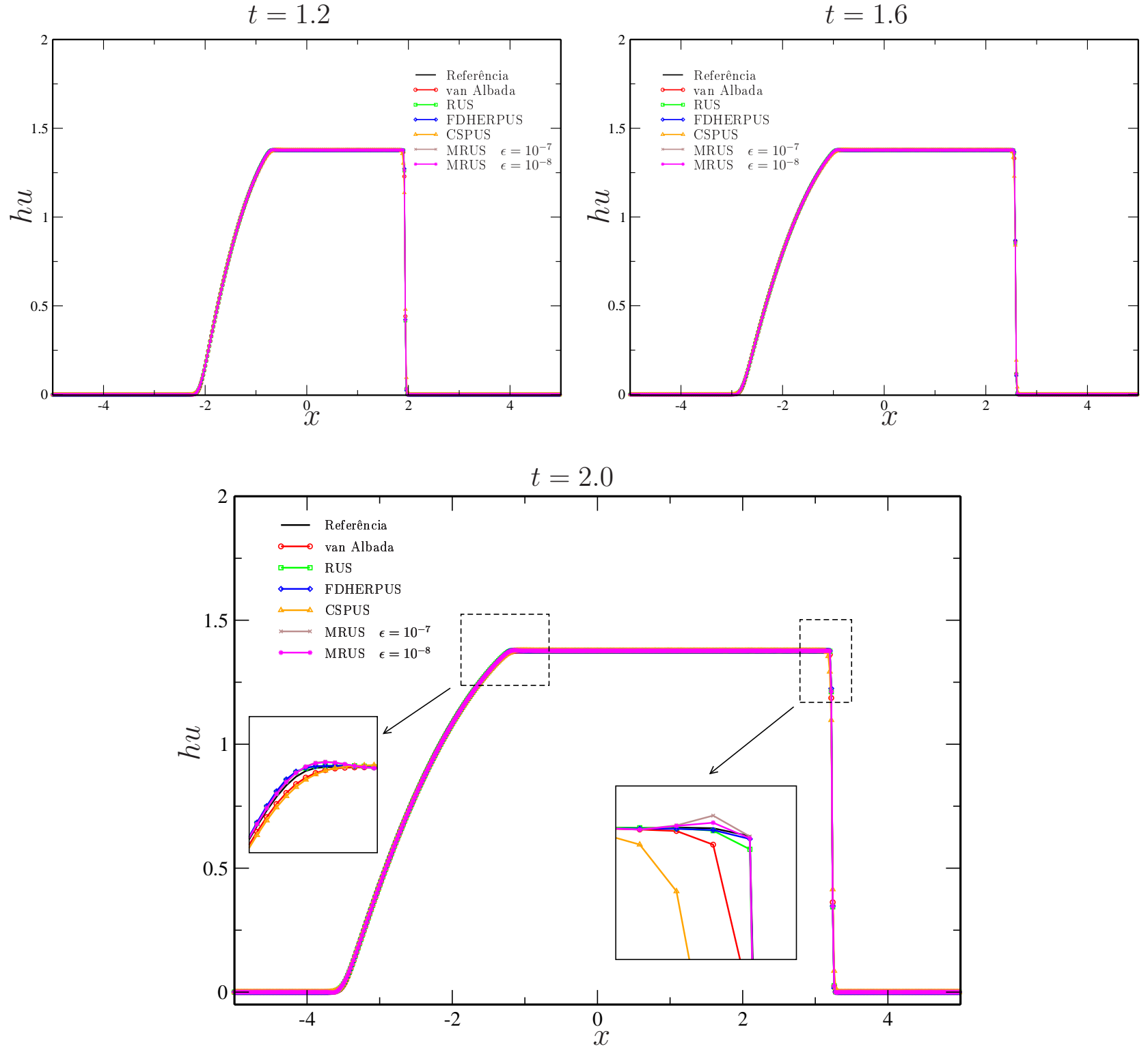

Figura 6.21: Evolução temporal para o problema de Dam-break. Ilustra-se as soluções exata e numérica na variável $h u$. 
Tabela 6.5: Erro relativo $E_{h}$ e estimativa para a ordem de convergência $q$.

\begin{tabular}{|c|c|c|c|}
\hline \multirow[t]{2}{*}{ Esquema } & \multirow[t]{2}{*}{ Malha } & \multicolumn{2}{|c|}{$L_{1}$} \\
\hline & & Erro & Ordem \\
\hline \multirow[t]{4}{*}{ van Albada } & 125 & 0.003744 & - \\
\hline & 250 & 0.002474 & 0.897939 \\
\hline & 500 & 0.001446 & 0.526098 \\
\hline & 1000 & 0.000776 & 0.745746 \\
\hline \multirow[t]{4}{*}{ RUS } & 125 & 0.003540 & - \\
\hline & 250 & 0.002295 & 0.850298 \\
\hline & 500 & 0.001368 & 0.620112 \\
\hline & 1000 & 0.000749 & 0.722851 \\
\hline \multirow[t]{4}{*}{ FDHERPUS } & 125 & 0.003458 & - \\
\hline & 250 & 0.002970 & 0.852336 \\
\hline & 500 & 0.001347 & 0.612039 \\
\hline & 1000 & 0.000732 & 0.754282 \\
\hline \multirow[t]{4}{*}{ CSPUS } & 125 & 0.005109 & - \\
\hline & 250 & 0.002992 & 0.907245 \\
\hline & 500 & 0.001640 & 0.796602 \\
\hline & 1000 & 0.000825 & 0.870520 \\
\hline \multirow[t]{4}{*}{ MRUS $\epsilon=10^{-8}$} & 125 & 0.003593 & 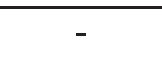 \\
\hline & 250 & 0.002321 & 0.850614 \\
\hline & 500 & 0.001378 & 0.622768 \\
\hline & 1000 & 0.000754 & 0.709497 \\
\hline \multirow[t]{4}{*}{ MRUS $\epsilon=10^{-9}$} & 125 & 0.003573 & - \\
\hline & 250 & 0.002316 & 0.847611 \\
\hline & 500 & 0.001379 & 0.617505 \\
\hline & 1000 & 0.000755 & 0.703237 \\
\hline
\end{tabular}



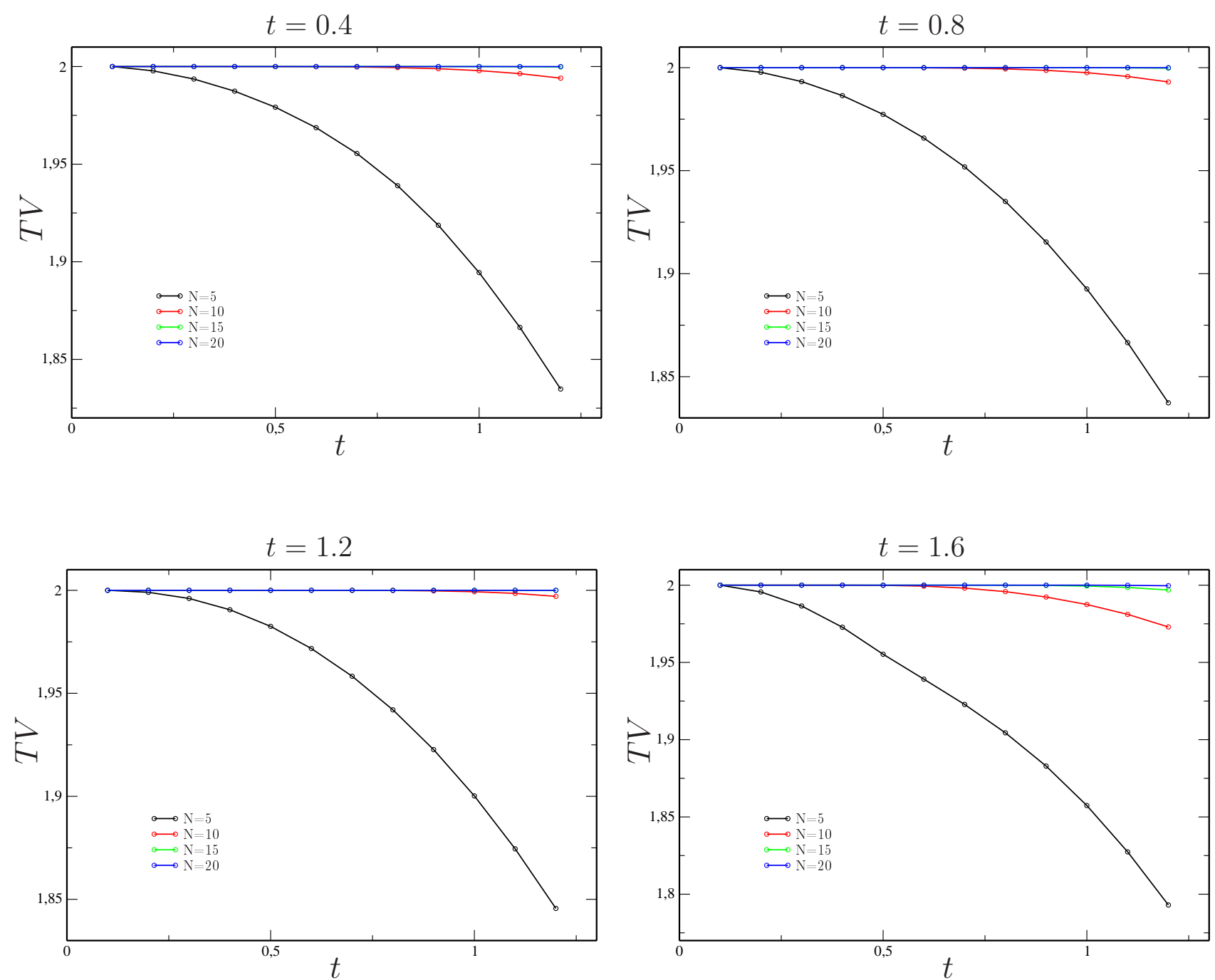

Figura 6.22: Análise TVD para o Teste 7 para os esquemas van Albada, RUS, FDHERPUS e CSPUS. 


\section{Resultados numéricos $2 D$ e 2D-1/2}

Neste capítulo é investigado o desempenho dos esquemas van Albada, FDHERPUS, CSPUS, RUS e MRUS para resolver problemas complexos de leis de conservação 2D (esquações de Euler e águas rasas). Fazendo-se o uso do esquema FDHERPUS são derivados também resultados numéricos para escoamentos incompressíveis de fluidos newtonianos envolvendo superfícies livres móveis (equações de Navier-Stokes).

\subsection{Equações de Euler}

Quatro problemas padrões em fluido dinâmica computacional modelados pelas equações de Euler (2.17) são simulados aqui: o primeiro refere-se a um teste de precisão usando condição inicial suave (equações de Euler sem termo fonte); o segundo constitui um problema interessante em astrofísica (equações de Euler sem termo fonte); o terceiro envolve o problema de interações de quatro choques (equações de Euler sem termo fonte); e o último teste simula as instabilidades de Rayleigh-Taylor (equações de Euler com termo fonte).

\section{Teste 1: Teste de precisão}

Neste teste é empregada a condição inicial (suave)

$$
\left\{\begin{array}{l}
\rho(x, y, 0)=1+0.2 \sin (\pi(x+y)), \\
u(x, y, 0)=0.7 \\
v(x, y, 0)=0.3 \\
p(x, y, 0)=1.0
\end{array}\right.
$$

a fim de mostrar a proximidade entre os resultados computacionais e a solução exata do problema (2.17)-(7.1). A solução exata transiente encontrada para a densidade $\rho$ (ver, por exemplo, 
[50]) é dada por

$$
\rho(x, y, t)=1+0.2 \sin (\pi(x+y-(u+v) t)), \quad(x, y) \in[0,2] \times[0,2] .
$$

Para a simulação, os seguintes dados foram considerados: uma malha de $N \times M=320 \times 320$ células computacionais, número de Courant $C=0.5$ e tempo final de simulação $t=2.0$. Os resultados das simulações podem ser vistos na Figura 7.1, onde observa-se que, no global, os resultados fornecidos pelos esquemas van Albada, RUS, FDHERPUS, CSPUS e MRUS foram muito semelhantes, com o esquema MRUS com parâmetro $\epsilon=10^{-7}$ fornecendo (ligeiramente) o melhor resultado. Essa informação pode também ser vista em mais detalhes na tabela 7.1, onde consta os erros e as ordens locais de precisão para a propriedade densidade $\rho$.

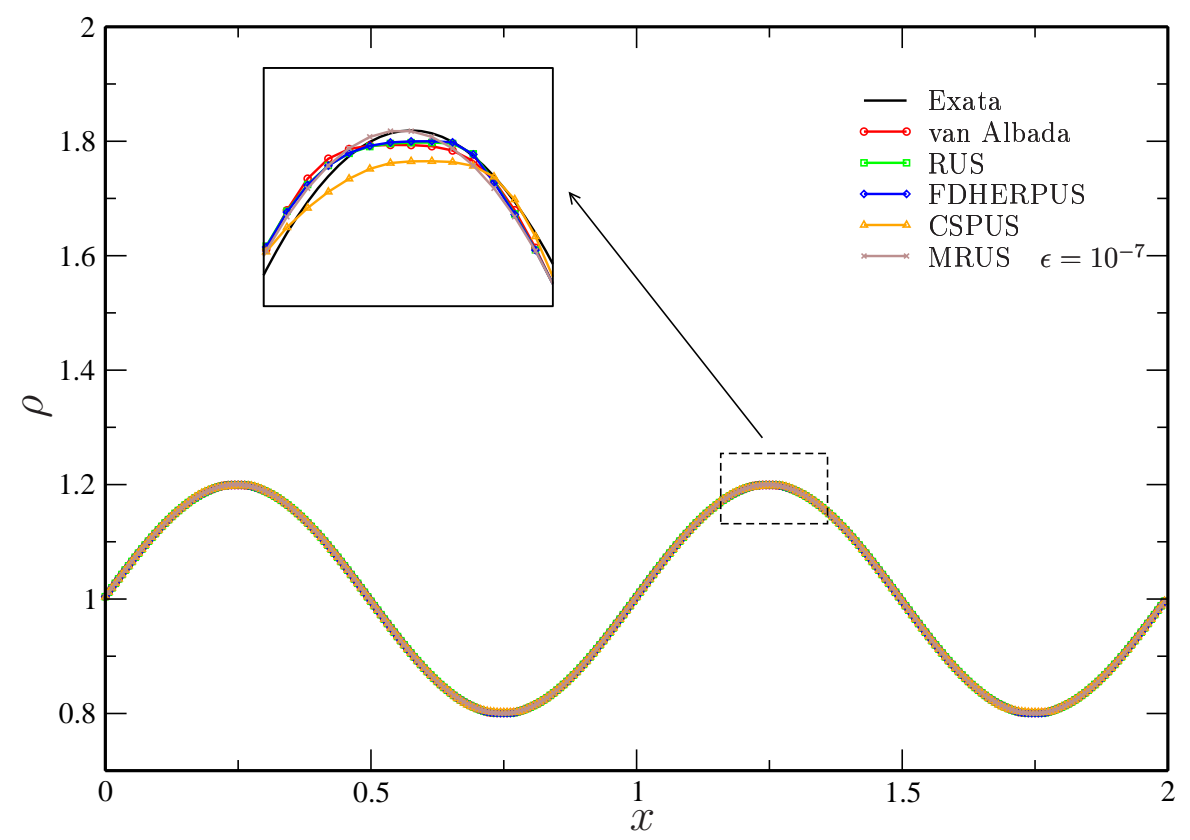

Figura 7.1: Aproximações obtidas com os esquemas van Albada, RUS, FDHERPUS, CSPUS e MRUS, $\operatorname{com} \epsilon=10^{-7}$

\section{Teste 2: Jatos astrofísicos}

As imagens obtidas pelo telescópio espacial Hubble revelam em detalhes ondas de choque em jatos astrofísicos (a baixas e a altas velocidade) e colisões das partículas do vento (gás) interestelar, isso motiva simular numericamente esses tipos de problemas. E o objetivo aqui é determinar se os esquemas van Albada, RUS, FDHERPUS, CSPUS e MRUS são capazes de simular com sucesso um problema particular da astrofísica proposto por Youngsoo (2003, 2005, 2008) $[26,28,27]$.

Na literatura é conhecido que para valores da velocidade, $u>2$, e mesmo a valores de 
Tabela 7.1: Erro relativo $E_{h}$ e estimativa para a ordem de convergência $q$.

\begin{tabular}{|c|c|c|c|}
\hline \multirow[t]{2}{*}{ Esquema } & \multirow[t]{2}{*}{ Malha } & \multicolumn{2}{|c|}{$L_{1}$} \\
\hline & & Erro & Ordem \\
\hline \multirow[t]{5}{*}{ van Albada } & 20 & 0.009234 & - \\
\hline & 40 & 0.002257 & 2.066 \\
\hline & 80 & 0.000568 & 2.052 \\
\hline & 160 & 0.000127 & 2.027 \\
\hline & 320 & 0.000084 & 2.015 \\
\hline \multirow[t]{5}{*}{ FDHERPUS } & 20 & 0.009075 & - \\
\hline & 40 & 0.002171 & 2.080 \\
\hline & 80 & 0.000520 & 2.051 \\
\hline & 160 & 0.000128 & 2.031 \\
\hline & 320 & 0.000039 & 2.018 \\
\hline \multirow[t]{5}{*}{ RUS } & 20 & 0.009136 & - \\
\hline & 40 & 0.002203 & 2.084 \\
\hline & 80 & 0.000527 & 2.063 \\
\hline & 160 & 0.000128 & 2.035 \\
\hline & 320 & 0.000039 & 2.020 \\
\hline \multirow[t]{5}{*}{ SCPUS } & 20 & 0.010826 & - \\
\hline & 40 & 0.002399 & 2.173 \\
\hline & 80 & 0.000554 & 2.114 \\
\hline & 160 & 0.000131 & 2.072 \\
\hline & 320 & 0.000032 & 2.040 \\
\hline \multirow[t]{5}{*}{ MRUS $\epsilon=10^{-7}$} & 20 & 0.009001 & - \\
\hline & 40 & 0.002021 & 2.046 \\
\hline & 80 & 0.000512 & 2.032 \\
\hline & 160 & 0.000102 & 2.007 \\
\hline & 320 & 0.000031 & 2.005 \\
\hline
\end{tabular}


$C$ consideravelmente reduzidos, o método de Godunov com termo de correção (incluindo um limitador de fluxo) não consegue simular bem este fenômeno (ver Youngsoo (2003) [26]). Isso constitui mais uma motivação para testar os esquemas propostos neste trabalho. A Figura 7.2 ilustra um jato astrofísico a velocidade (adimensional) $u=30$, mostrando vários tipos de ondas de choque no problema.

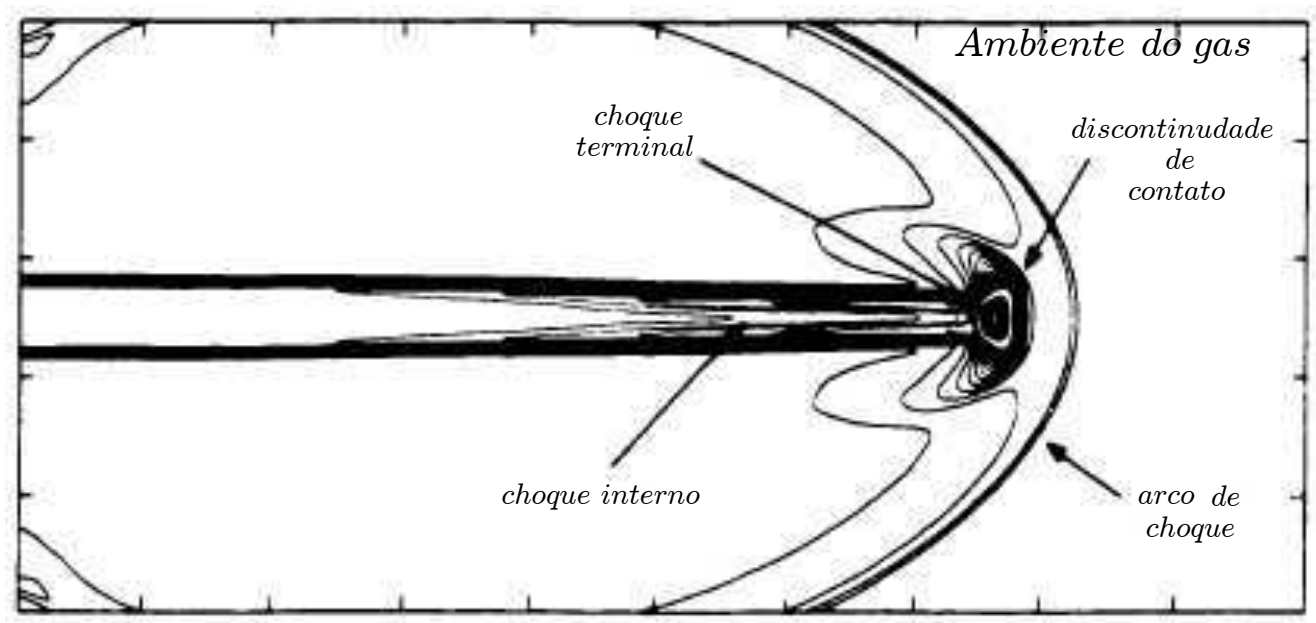

Figura 7.2: Jato astrofísico propagando-se num ambiente a velocidade adimensional $u=30$.

$\mathrm{Na}$ sequência apresenta-se simulações de um jato astrofísico via método de Godunov com termo de correção de alta ordem e com limitadores de fluxo correspondentes aos esquemas minmod (a referência numa malha com $800 \times 400$ células computacionais), van Albada, RUS, FDHERPUS, CSPUS e MRUS. Os dados empregados nas simulações foram: malha de $400 \times 200$ células computacionais, domínio computacional $[0,2] \times[0,1]$, número de Courant $C=0.1 \mathrm{e}$ tempo final de simulação $t=1.0$. As condições de entrada (inflow) e as condições dentro do domínio (ambiente) foram prescritas, respectivamente, como

$$
\left[\begin{array}{l}
\rho \\
u \\
v \\
p
\end{array}\right]=\left[\begin{array}{c}
5.0 \\
2.0 \\
0.0 \\
0.4127
\end{array}\right], \quad\left[\begin{array}{l}
\rho \\
u \\
v \\
p
\end{array}\right]=\left[\begin{array}{c}
0.5 \\
0.0 \\
0.0 \\
0.4127
\end{array}\right]
$$

As Figuras 7.3 - 7.8 mostram os campos de densidade (casos (a)) e pressão (casos (b)) calculados com os esquemas van Albada, RUS, FDHERPUS, CSPUS e MRUS com $\epsilon=10^{-7}$, onde pode-se observar que os esquemas numéricos foram capazes de simular o problema, confrontando bem com os resultados fornecido pelo esquema minmod e os dados de Youngsoo (2003) [26].

Na Figura 7.9 estão apresentados os valores da densidade $\rho$, ao longo da reta $y=0.5$, obtidos com os esquemas van Albada, RUS, FDHERPUS, CSPUS e MRUS mais a referência (minmod). No geral, pode-se inferir que os esquemas FDHERPUS, MRUS $\epsilon=10^{-7}$ e van Albada forneceram (nesta ordem de classificação) os melhores resultados. Os piores resultados 
(a)

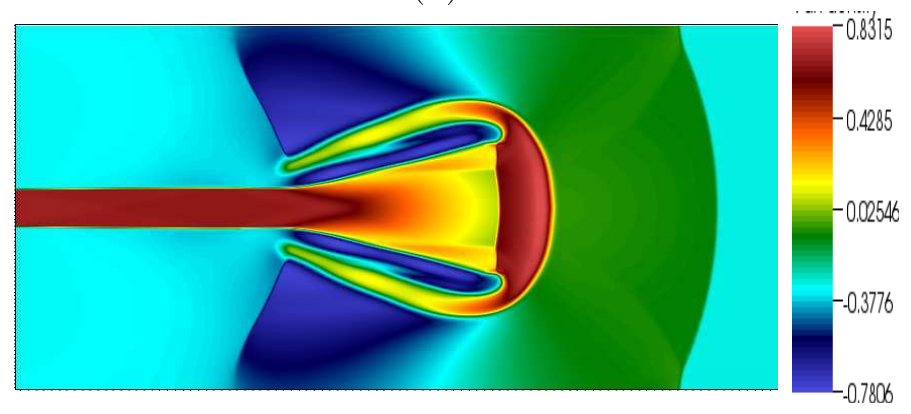

(b)

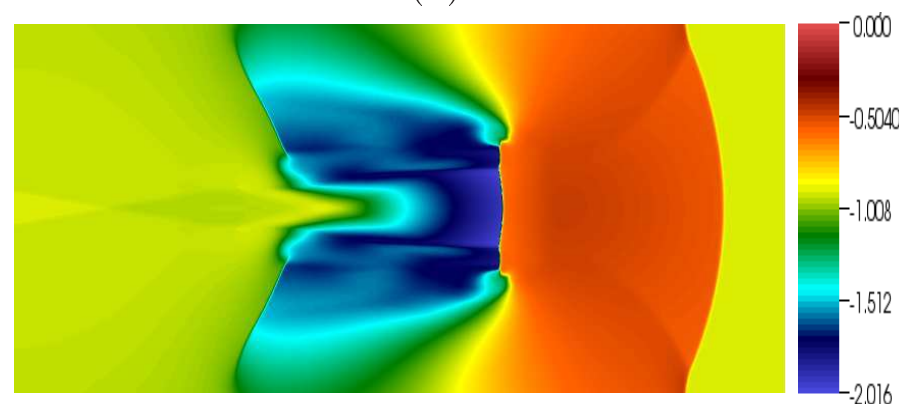

Figura 7.3: Resultados numéricos obtidos com o uso do esquema minmod.

(a)

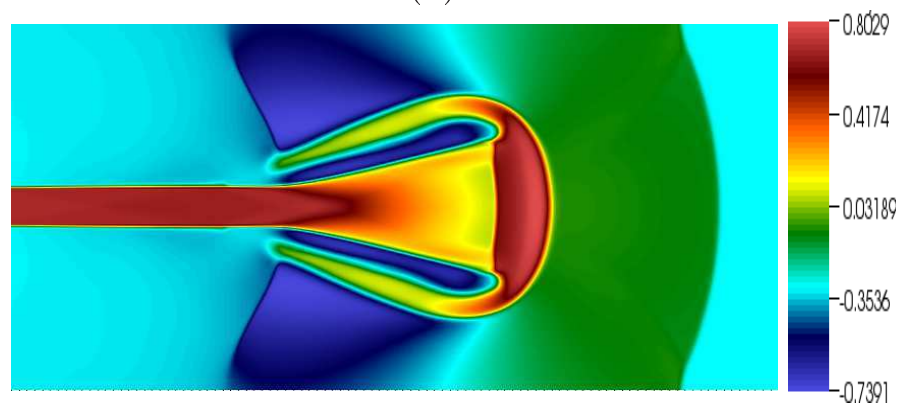

(b)

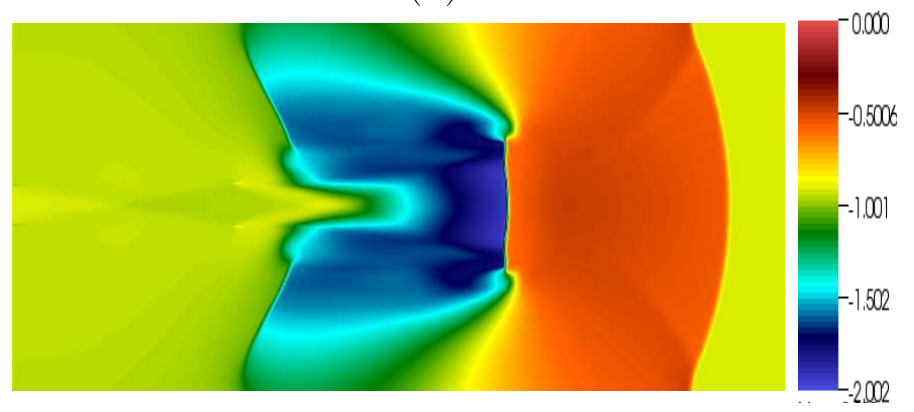

Figura 7.4: Resultados numéricos obtidos com o uso do esquema van Albada. 
(a)

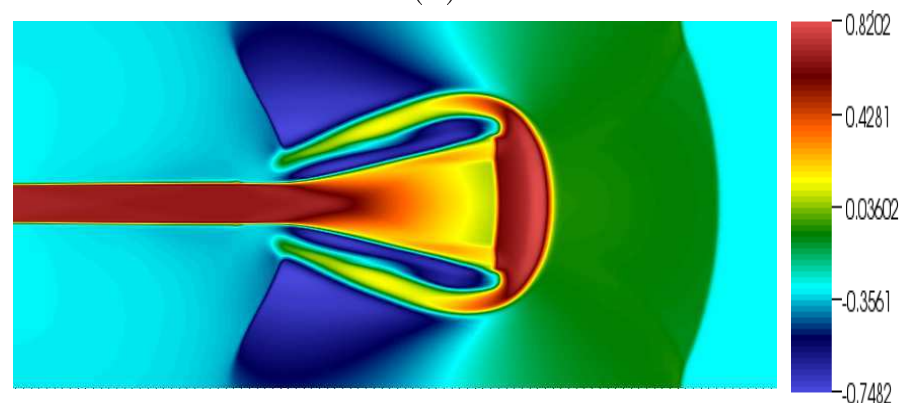

(b)

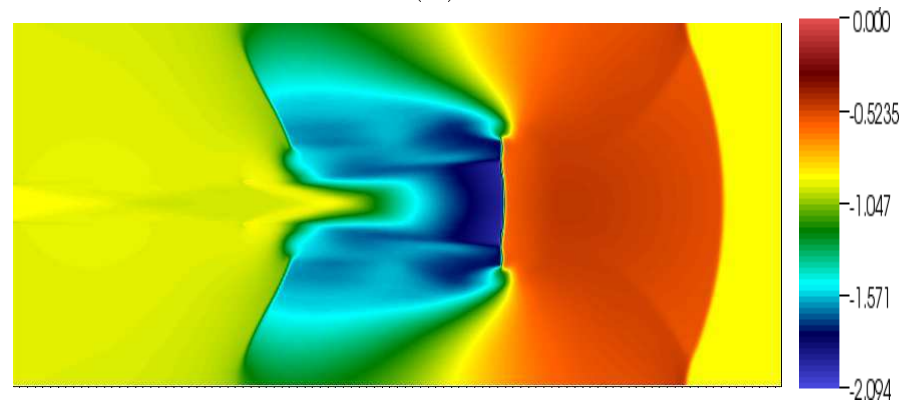

Figura 7.5: Resultados numéricos obtidos com o uso do esquema FDHERPUS.

(a)

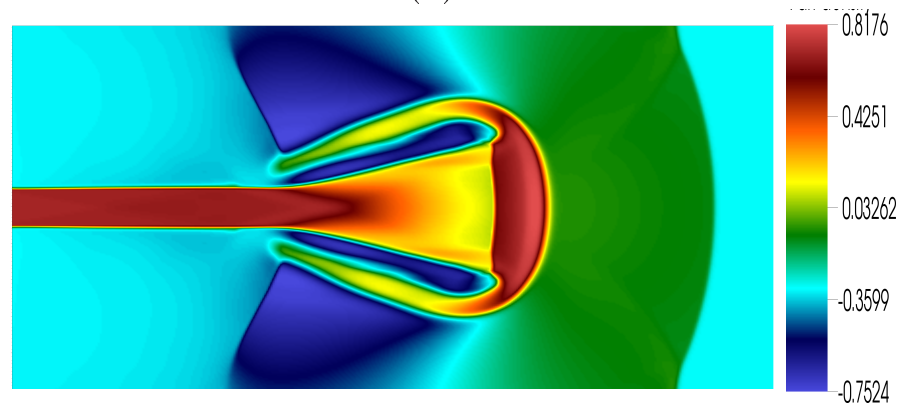

(b)

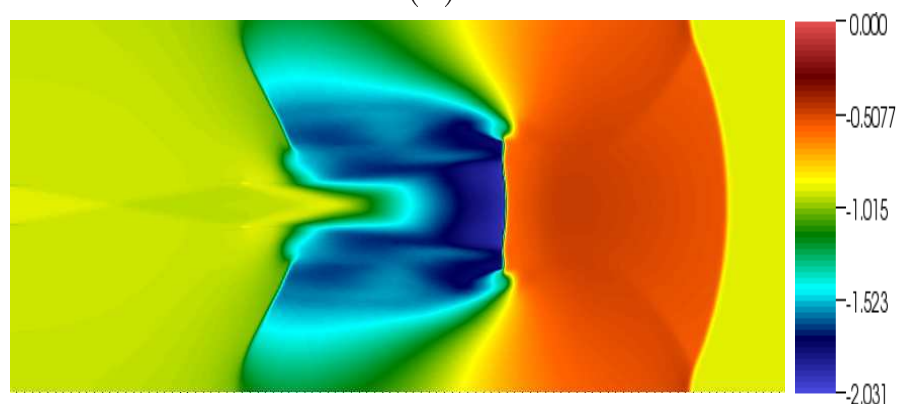

Figura 7.6: Resultados numéricos obtidos com o uso do esquema RUS. 
(a)

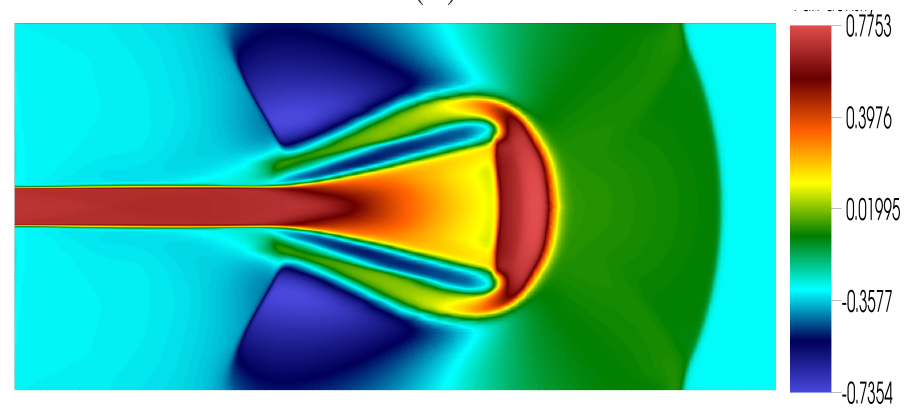

(b)

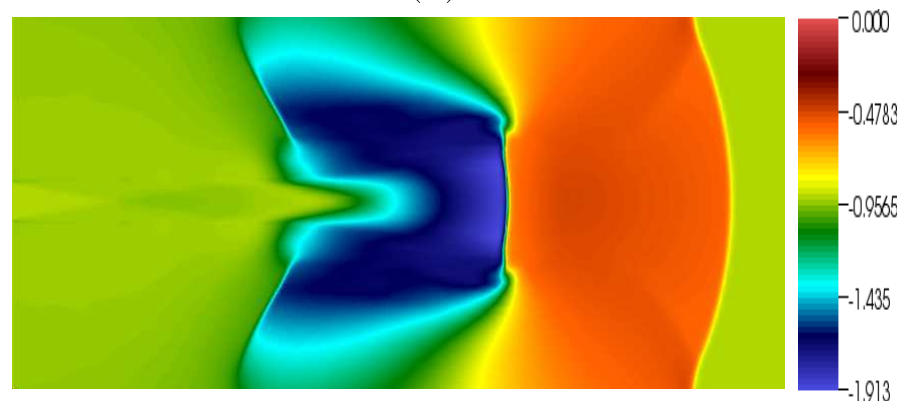

Figura 7.7: Resultados numéricos obtidos com o uso do esquema CSPUS.

(a)

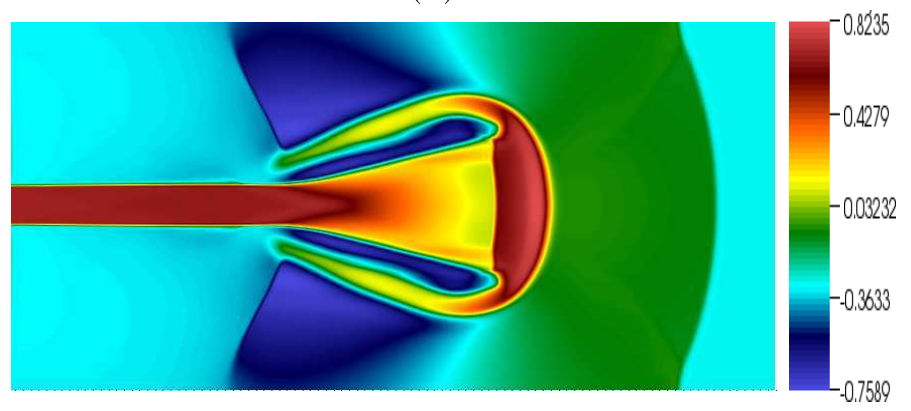

(b)

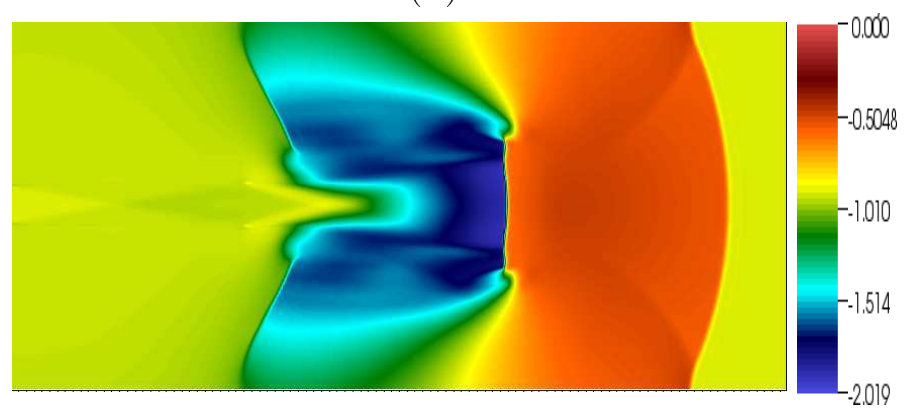

Figura 7.8: Resultados numéricos obtidos com o uso do esquema MRUS. 
foram obtidos com o uso do esquema CSPUS.

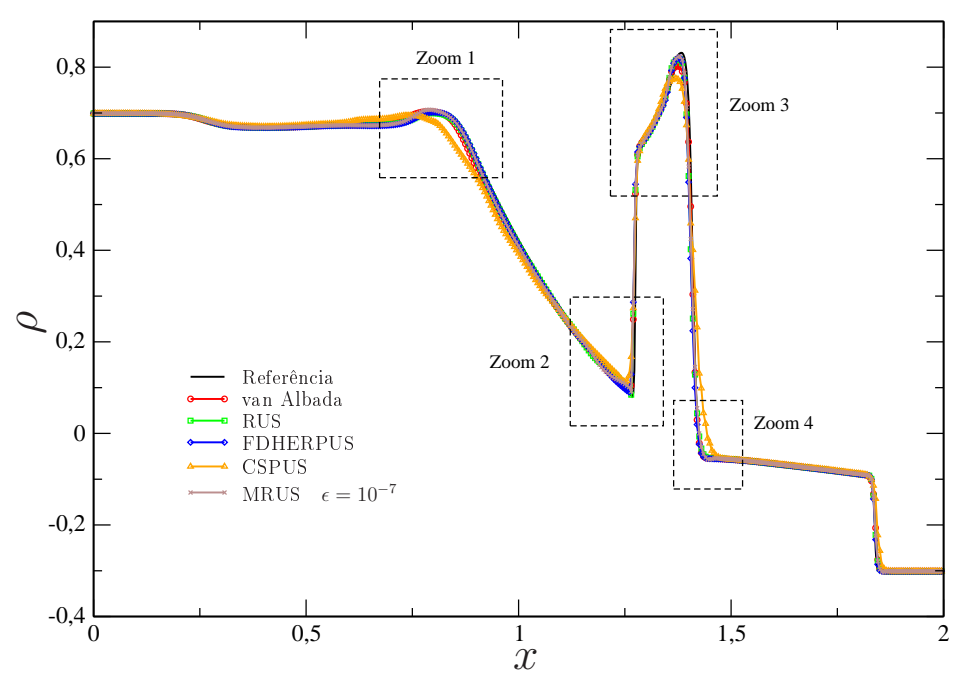

\section{Zoom 1}

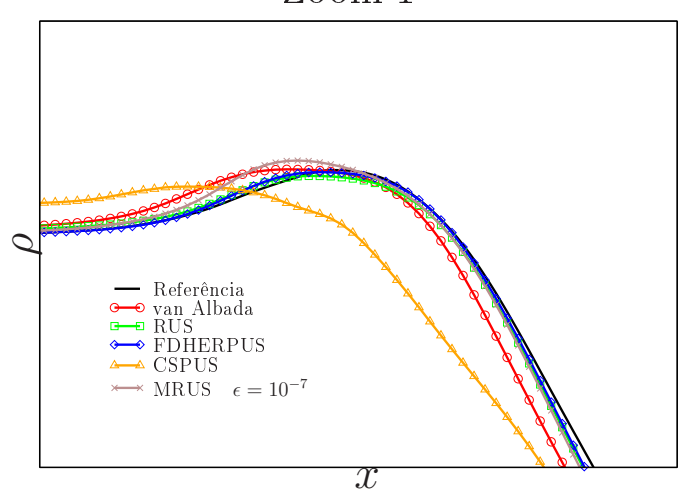

Zoom 3

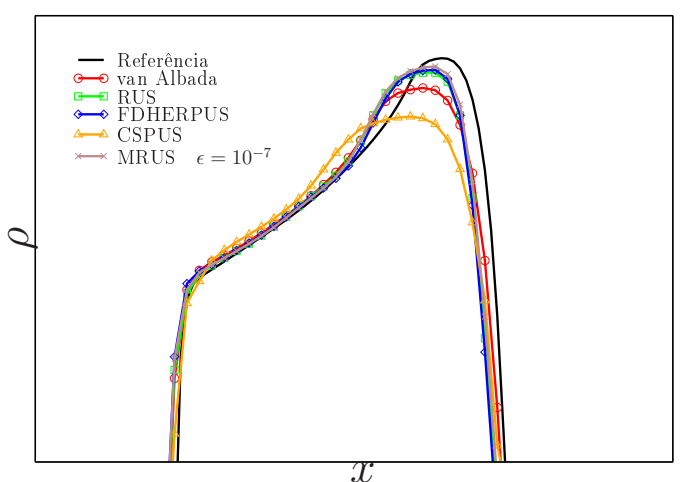

Zoom 2

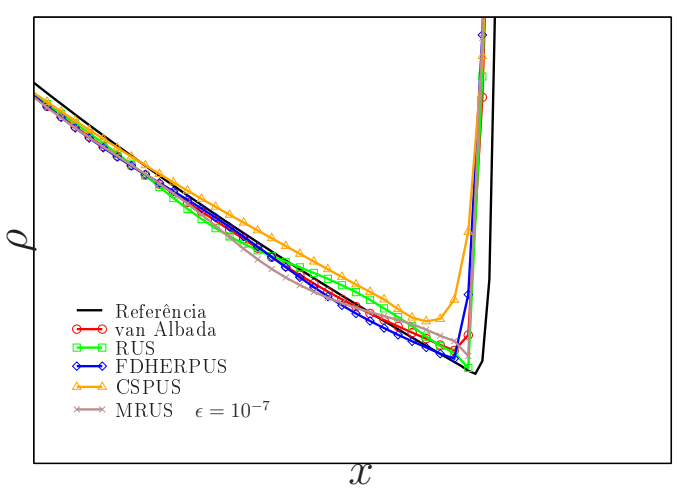

Zoom 4

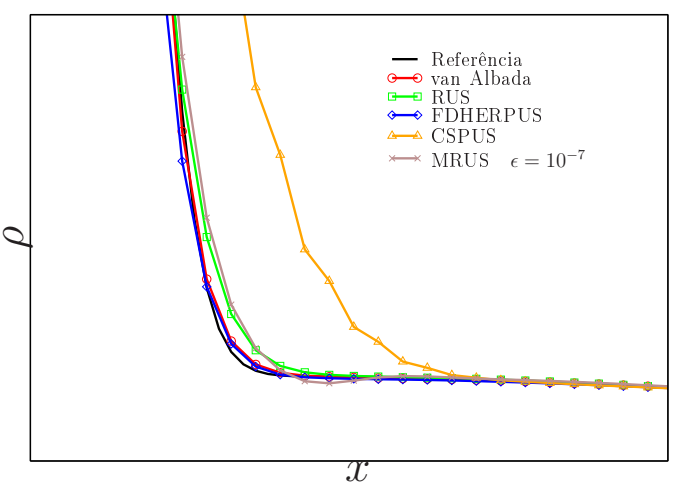

Figura 7.9: Densidade $\rho$ ao longo da reta $y=0.5$ para o problema do jato astrofísico.

\section{Teste 3: Interação entre quatro choques}

O problema da interação entre quatro choques, originalmente proposto por Collins (1993) [64], é caracterizado pelo contato inicial de quatro ondas de choque no ponto $(0.8,0.8)$; e 
quando o tempo avança essa interação gera estruturas complexas que são difíceis de simular numericamente. O problema é útil para medir (qualitativamente) a introdução intrínseca de viscosidade numérica associada ao esquema convectivo. O problema foi resolvido no domínio $(x, y) \in[0,1] \times[0,1]$, com estados iniciais diferentes em cada quadrante dados por

$$
\left(\rho_{0}, u_{0}, v_{0}, p_{0}\right)^{T}= \begin{cases}(1.5,0,0,1.5)^{T}, & {[0.8,1] \times[0.8,1],} \\ (0.5323,1.206,0,0.3)^{T}, & {[0,0.8] \times[0.8,1],} \\ (0.138,1.206,1.206,0.029)^{T}, & {[0,0.8] \times[0,0.8],} \\ (0.5323,0,1.206,0.029)^{T}, & {[0.8,1] \times[0,0.8]}\end{cases}
$$

Os cálculos foram feitos usando o método de Godunov com termo de correção e os limitadores de fluxo correspondentes aos esquemas MC, van Albada, RUS, FDHERPUS, CSPUS e MRUS. O esquema MC foi selecionado como o de referência, uma vez que esse limitador tem sido um dos mais utilizados em aplicações de engenharia (ver Godoy (2010) [24]). Os dados considerados nas simulações foram: uma malha de $200 \times 200$ células computacionais, número de Courant $C=0.8$ e tempo final de simulação $t=0.8$.

Na Figura 7.10 são mostradas as simulações na malha $200 \times 200$ para o campo de densidade $\rho$ usando os diferentes esquemas. Como pode ser visto nesta Figura, todos os esquemas selecionados forneceram resultados semelhantes e bastantes dissipativos. Para ver qual esquema forneceu o melhores resultados, extraiu-se o perfil de $\rho$ ao longo da reta $y=x$; esses dados estão apresentados nas Figuras 7.11-7.1. Por estas Figuras pode ser visto que ambos os esquemas FDHERPUS e MRUS com $\epsilon=10^{-7}$ produziram os melhores resultados. O esquema van Albada ficou em quarta posição na classificação geral.

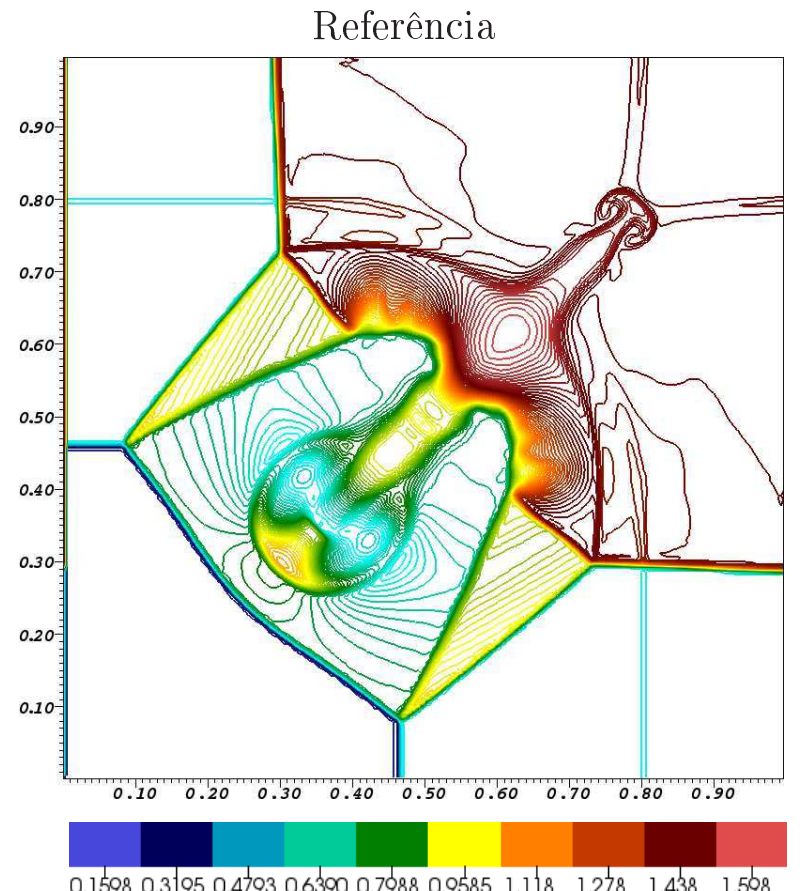

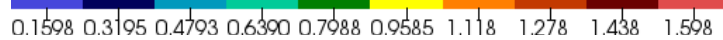

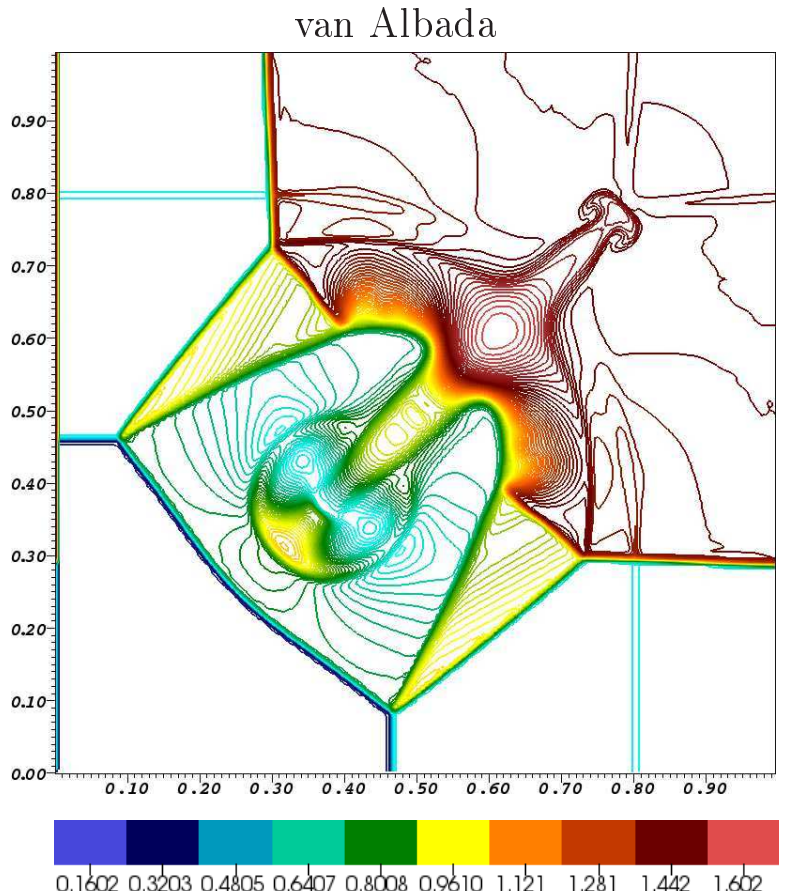

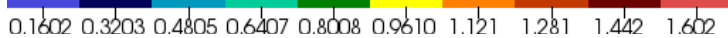


FDHERPUS

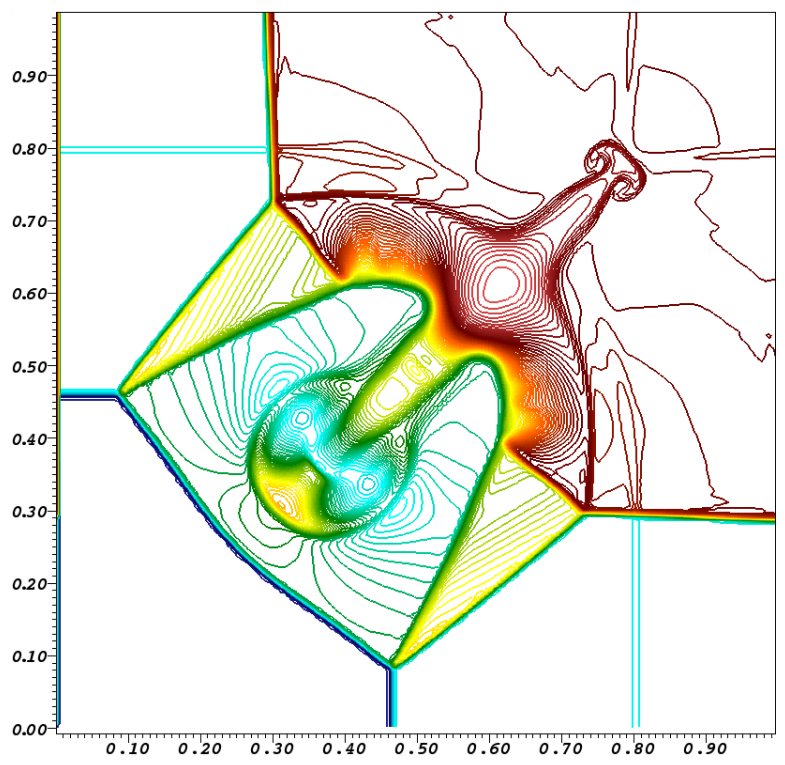

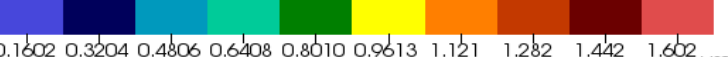
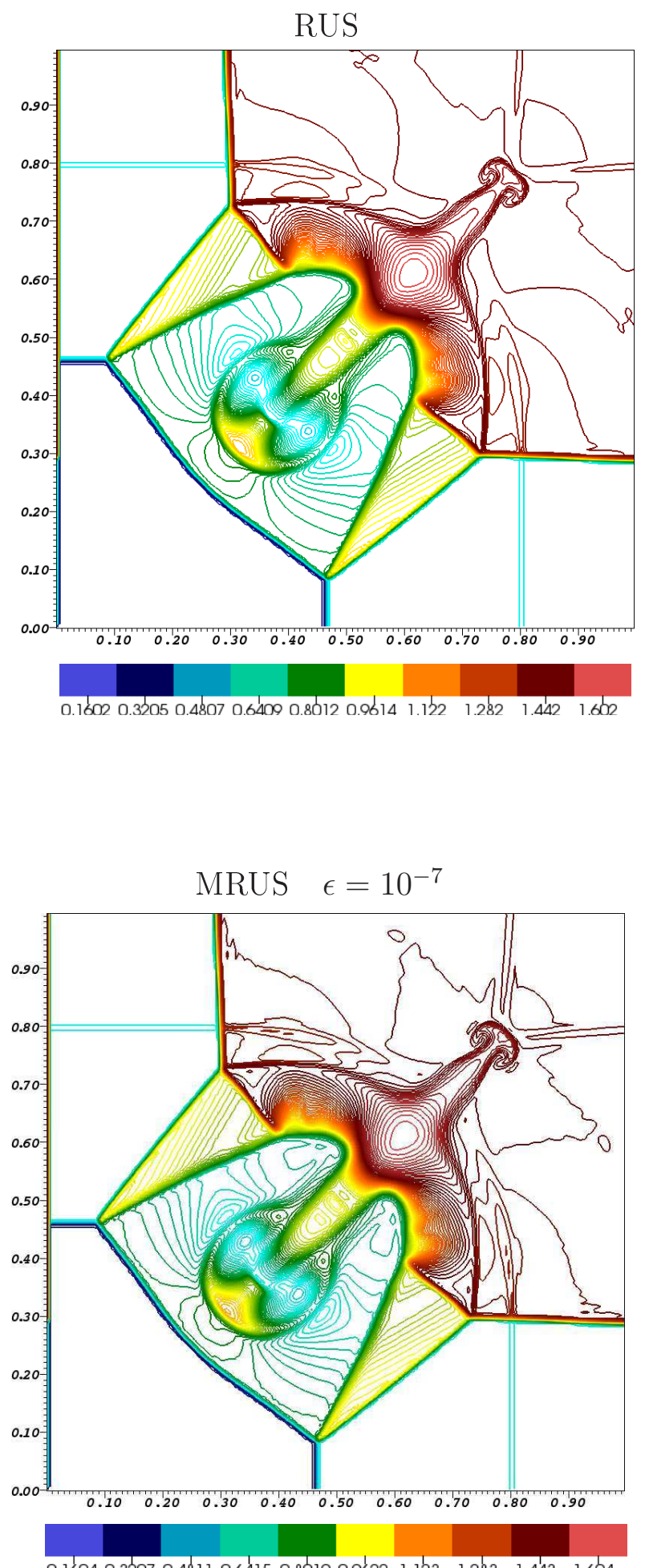

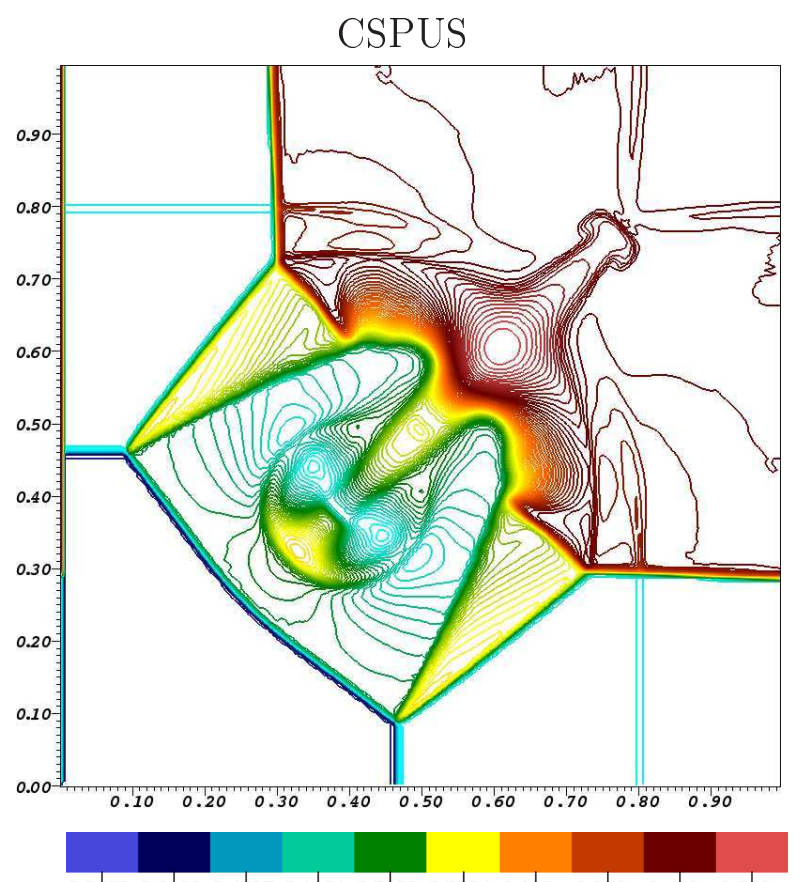

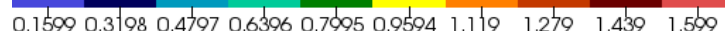

Figura 7.10: Contorno da densidade para o problema de interação entre quatro choques usando $200 \times 200$ células computacionais. 


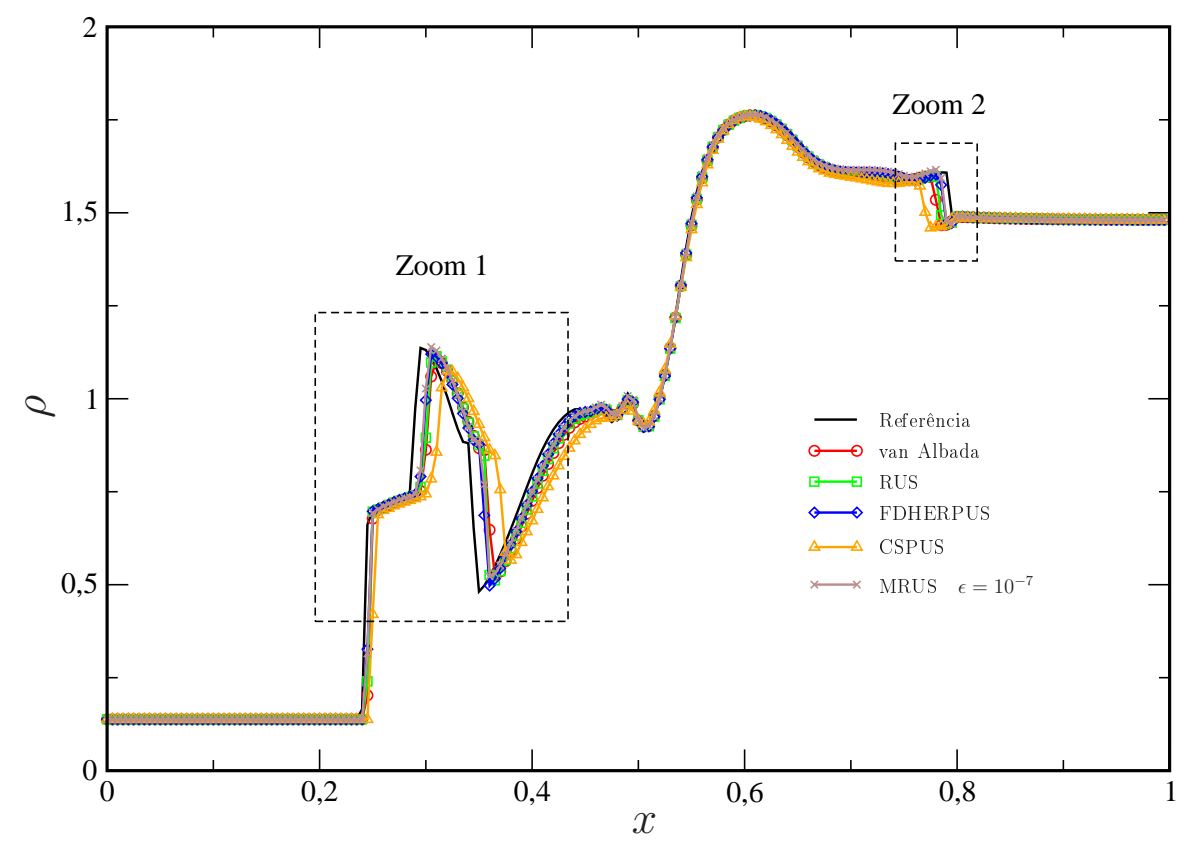

Figura 7.11: Contorno da densidade $\rho$ ao longo da reta $y=x$ para problema de interação entre quatro choques mostrando regiões de ampliação.

Zoom 1

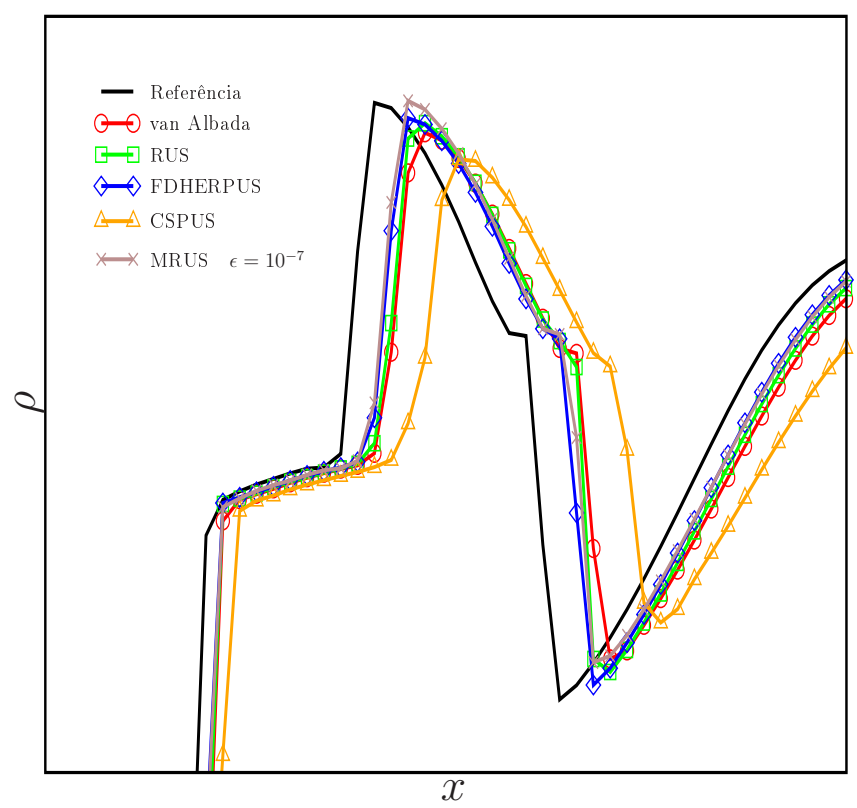

Zoom 2

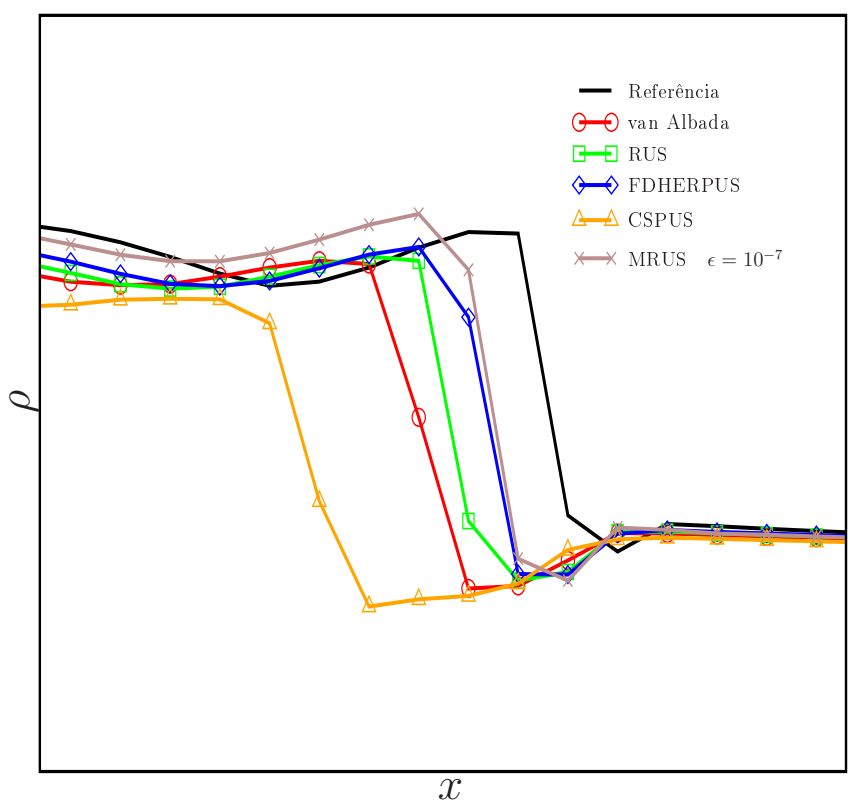

Figura 7.12: Regiões de ampliação da Figura 7.11.

Com o objetivo de investigar qual esquema introduz menos viscosidade numérica (erros intrinsicamente associados), o problema da interação entre quatro choques foi resolvido novamente numa malha mais fina contendo $1000 \times 1000$ células, da mesma forma como foi feito 
por Cada (2009) [9]. Os resultados dessas simulações estão apresentados na Figura 7.13, onde pode-se perceber claramente que os esquemas que introduzem menos viscosidade artificial foram FDHERPUS e MRUS, com o esquema MRUS introduzindo ligeiramente menos viscosidade que o esquema FDHERPUS. Os outros esquemas, incluindo o famoso van Albada, produziram, praticamente, os mesmos resultados.

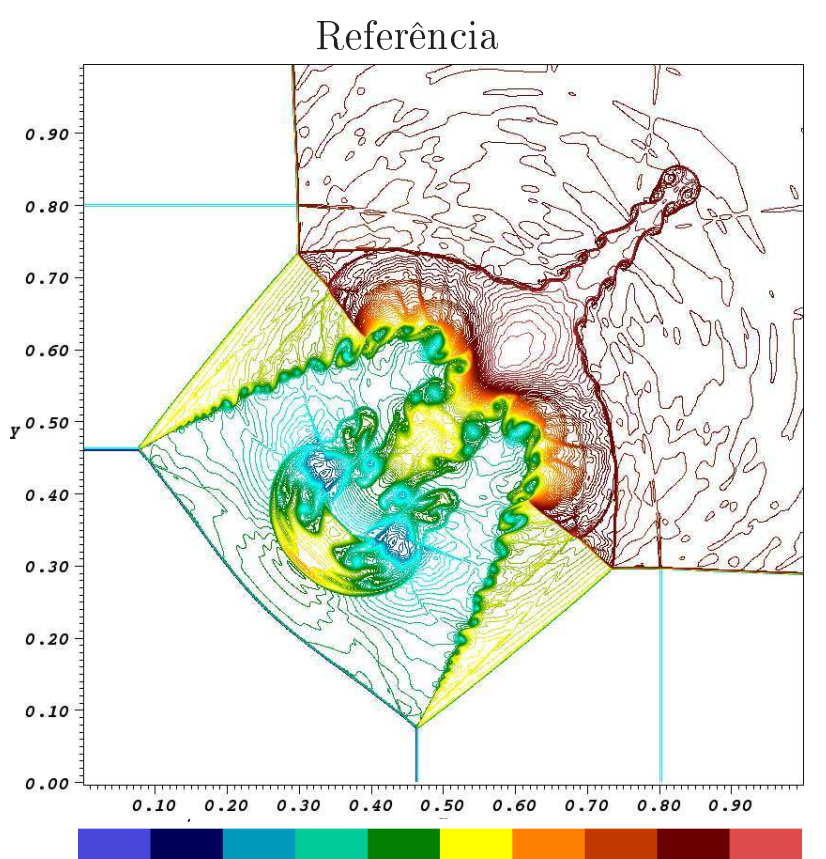

$0.16130 .32260 .48390 .64510 .80640 .9677 \quad 1.129 \quad 1.290 \quad 1.452 \quad 1.613$
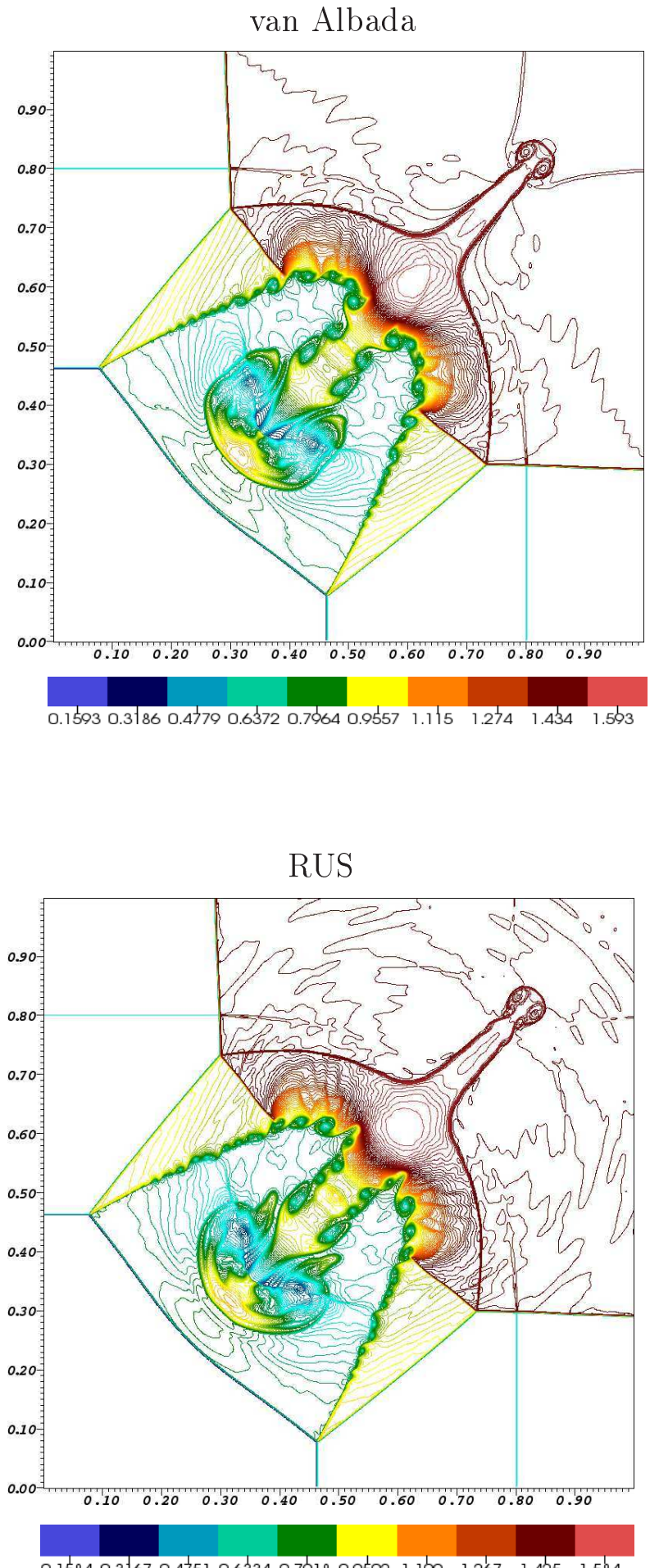

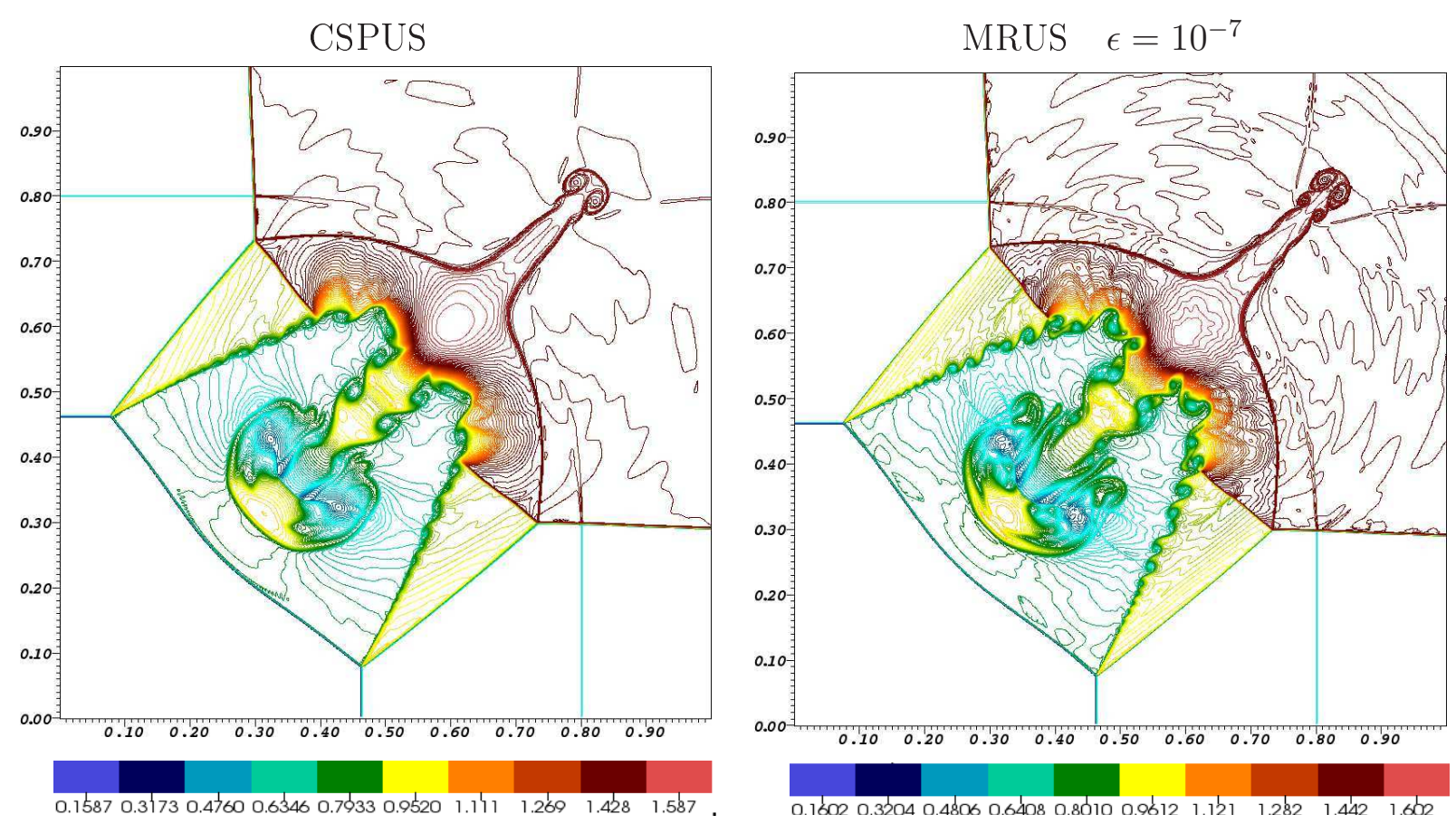

Figura 7.13: Contorno da densidade $\rho$ para o problema de interação entre quatro choques usando a malha de $1000 \times 1000$ células computacionais.

\section{Teste 4: Instabilidades de Rayleigh-Taylor}

Neste teste, investiga-se o desempenho dos esquemas desenvolvidos neste trabalho no problema conhecido como instabilidades de Rayleigh-Taylor (RT). Estas instabilidades estão presentes em uma grande variedade de fenômenos astrofísicos e geofísicos, como por exemplo explosões de supernovas. O problema pode ser modelado pelas equações (compressíveis) de Euler 2D (2.17) com termo fonte $\boldsymbol{S}(\boldsymbol{U})=(0,0, \rho g, \rho v g)^{T}$. Com as equações de Euler, as estruturas complexas de pequenas escalas são produzidas pela viscosidade numérica (intrínseca) dos esquemas, e não pela viscosidade do material. As instabilidades de RT ocorrem, por exemplo, quando há superposição de dois fluidos (um mais pesado e um mais leve) sob a ação de um campo gravitacional. Perturbações iniciais na interface entre os dois fluidos são amplificadas e crescem com o tempo, levando à formação de bolhas e espigões, cada um dos quais penetrando uns nos outros, seguido do desenvolvimento de uma camada de mistura altamente complexa. O problema representa um importante teste para esquemas de alta resolução upwind e que pode ser utilizado para medir a introdução intrínseca de viscosidade numérica artificial.

As equações de Euler (2.17) são resolvidas numericamente usando o algoritmo de propagação de ondas (uma variante do método de Godunov com termo de correção associado aos limitadores van Albada, FDHERPUS, CSPUS, RUS e MRUS), implementado no ambiente de simulação CLAWPACK de LeVeque (2004) [41]. O problema das instabilidades de Rayleigh-Taylor é definido no domínio $\left[0, \frac{1}{4}\right] \times[0,1]$, e as condições iniciais são tomadas de Shu (2003) [62]. No 
tempo $t=0$, a interface entre os dois gases está em $y=\frac{1}{2}$. O fluido mais denso está abaixo da interface, $\operatorname{com} \rho=2$, e o menos denso está acima da interface, com $\rho=1$, como mostra a Figura 7.14. O movimento se dá no sentido de y positivo. A pressão é contínua em toda interface e é imposta uma pequena perturbação na velocidade do fluido na direção $y$. Em suma, as condições iniciais são como seguem

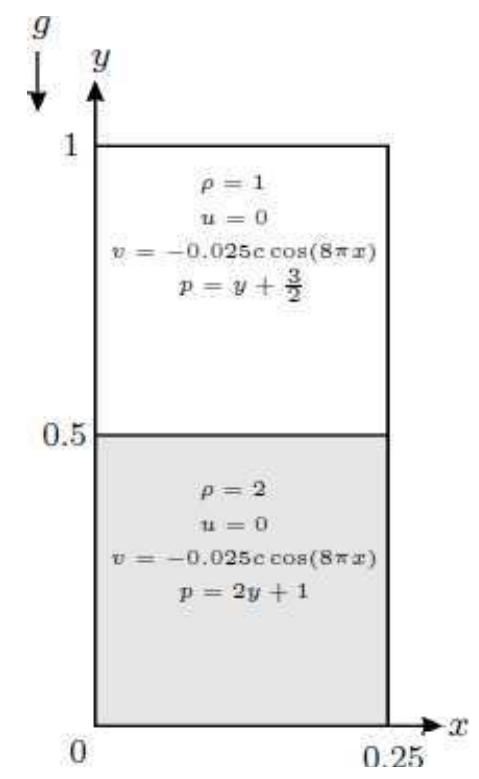

Figura 7.14: Domínio e condições iniciais para problema de RT.

$$
\left(\rho_{0}, u_{0}, v_{0}, p_{0}\right)^{T}= \begin{cases}(2,0,-0.025 c \cdot \cos (8 \pi x), 2 y+1)^{T}, & 0 \leq y<\frac{1}{2}, \\ \left(1,0,-0.025 c \cdot \cos (8 \pi x), y+\frac{3}{2}\right)^{T}, & \frac{1}{2} \leq y<1,\end{cases}
$$

onde $c=\sqrt{\frac{\gamma p}{\rho}}$ é a velocidade do som. As condições de contorno impostas são: nas fronteiras direita e esquerda é usada condição reflexiva; no contorno superior impõe-se $\rho=1, p=2.5, u=$ $0, v=0$; e no inferior toma-se $\rho=2, p=1, u=0, v=0$. A constante gravitacional utilizada é $g=1$.

As soluções numéricas foram calculadas até o tempo final $t=1.95$, com $C=0.6$, e em quatro malhas de $60 \times 240,120 \times 480,240 \times 960$ e $480 \times 1920$ células. Como neste caso não se conhece a solução analítica do problema, os resultados numéricos são comparados (qualitativamente) com aqueles obtidos via os limitadores van Albada e WENO5 [62]. Os resultados das simulações com os diferentes limitadores estão apresentados nas Figuras 7.15 e 7.16, onde pode-se ver diferenças significativas entre os resultados produzidos pelos diferentes limitadores. Em particular, quando comparado com os resultados fornecidos pelo esquema WENO5, o esquema FDHERPUS capturou melhor as estruturas vorticais e o esquema MRUS com $\epsilon=10^{-7}$ produziu os piores resultados. O esquema van Albada mostrou-se bastante dissipativo neste problema. 

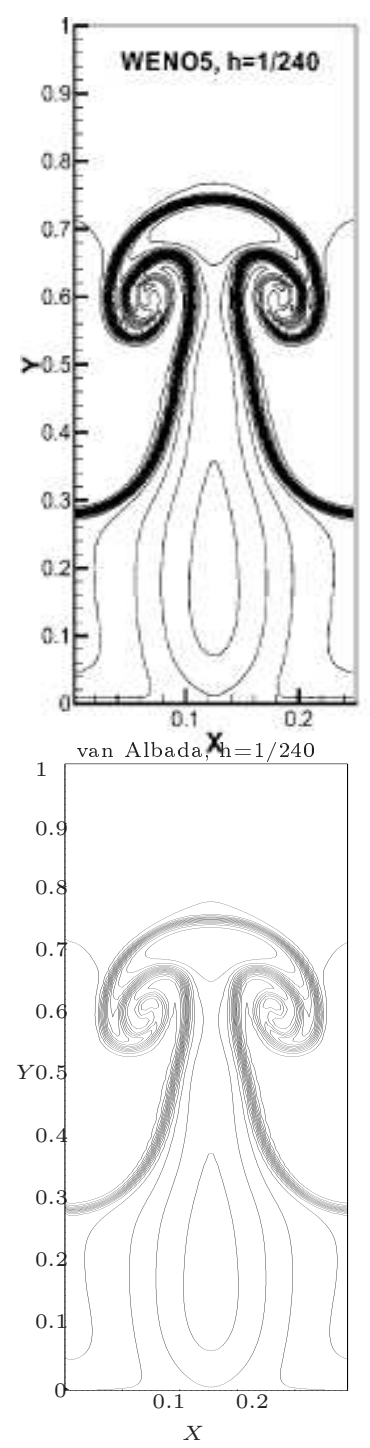

FDHERPUS, $\mathrm{h}=1 / 240$

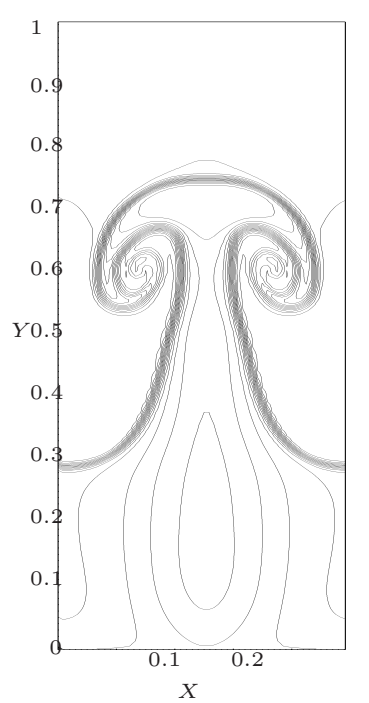

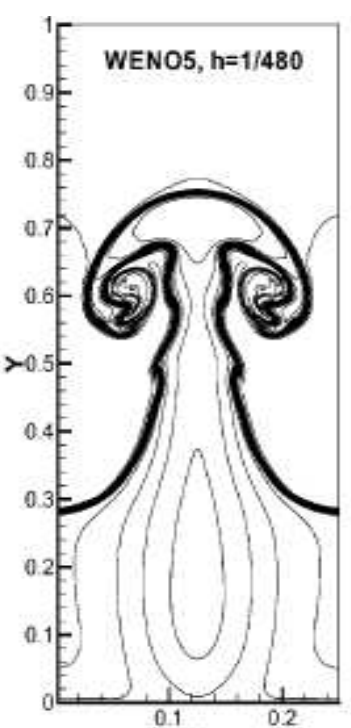

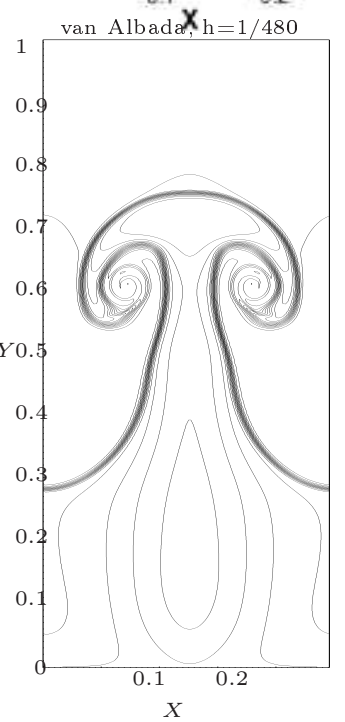

FDHERPUS, $\mathrm{h}=1 / 480$

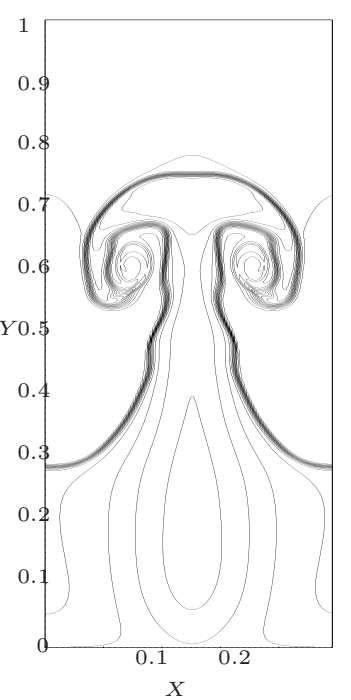

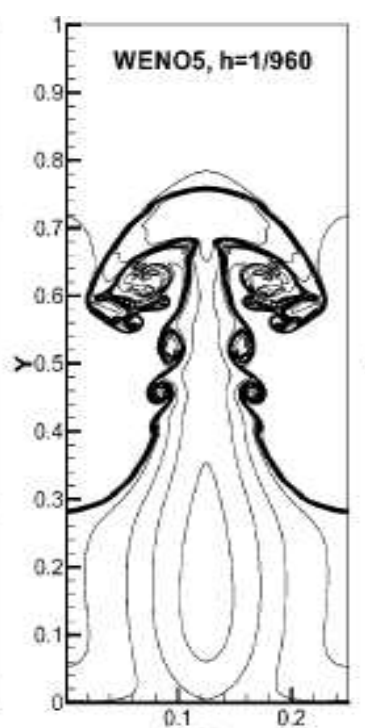

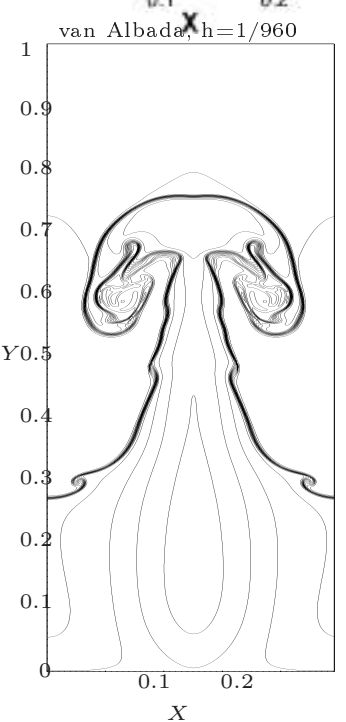

FDHERPUS, $\mathrm{h}=1 / 960$

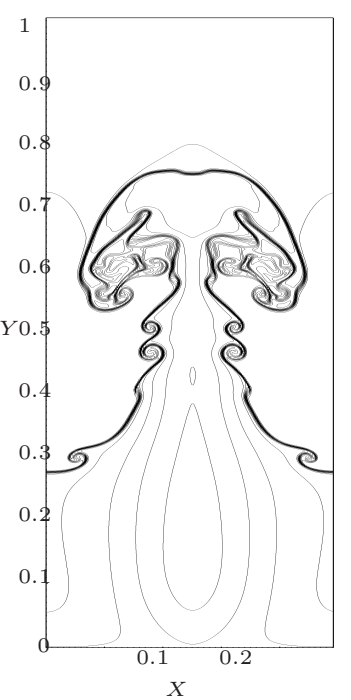

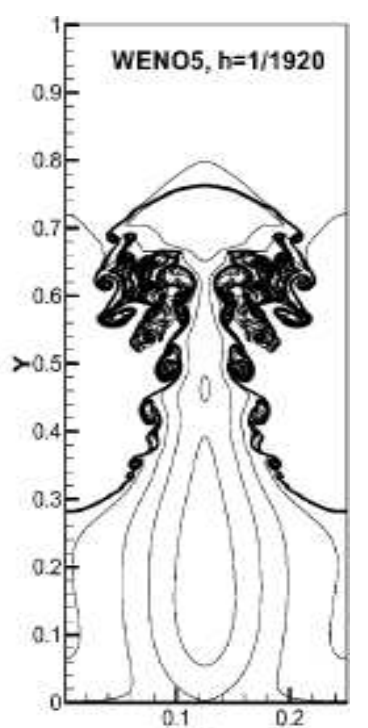

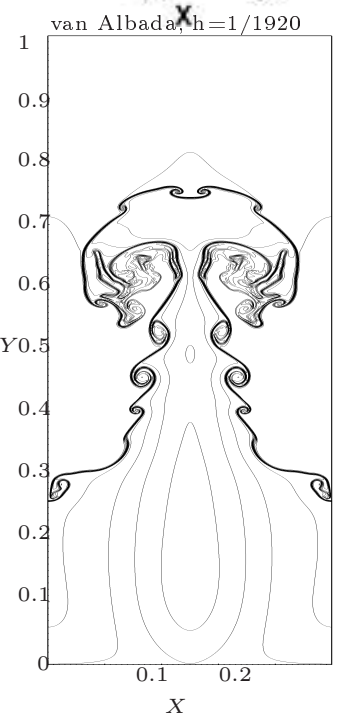

FDHERPUS, $\mathrm{h}=1 / 1920$

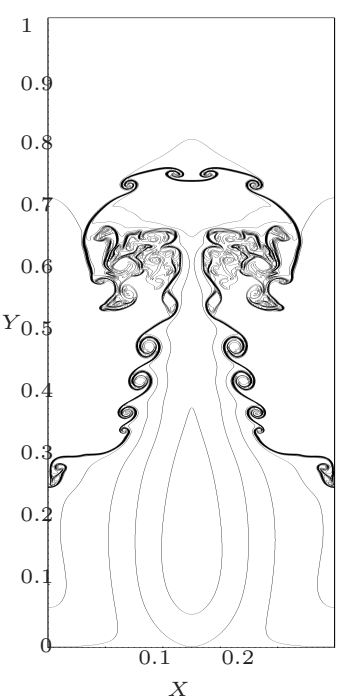

Figura 7.15: Contornos da densidade para o problema das instabilidades de Raylegh-Taylor. Linhas de contorno vão de $\rho=0.952269$ até $\rho=2.14589$. 

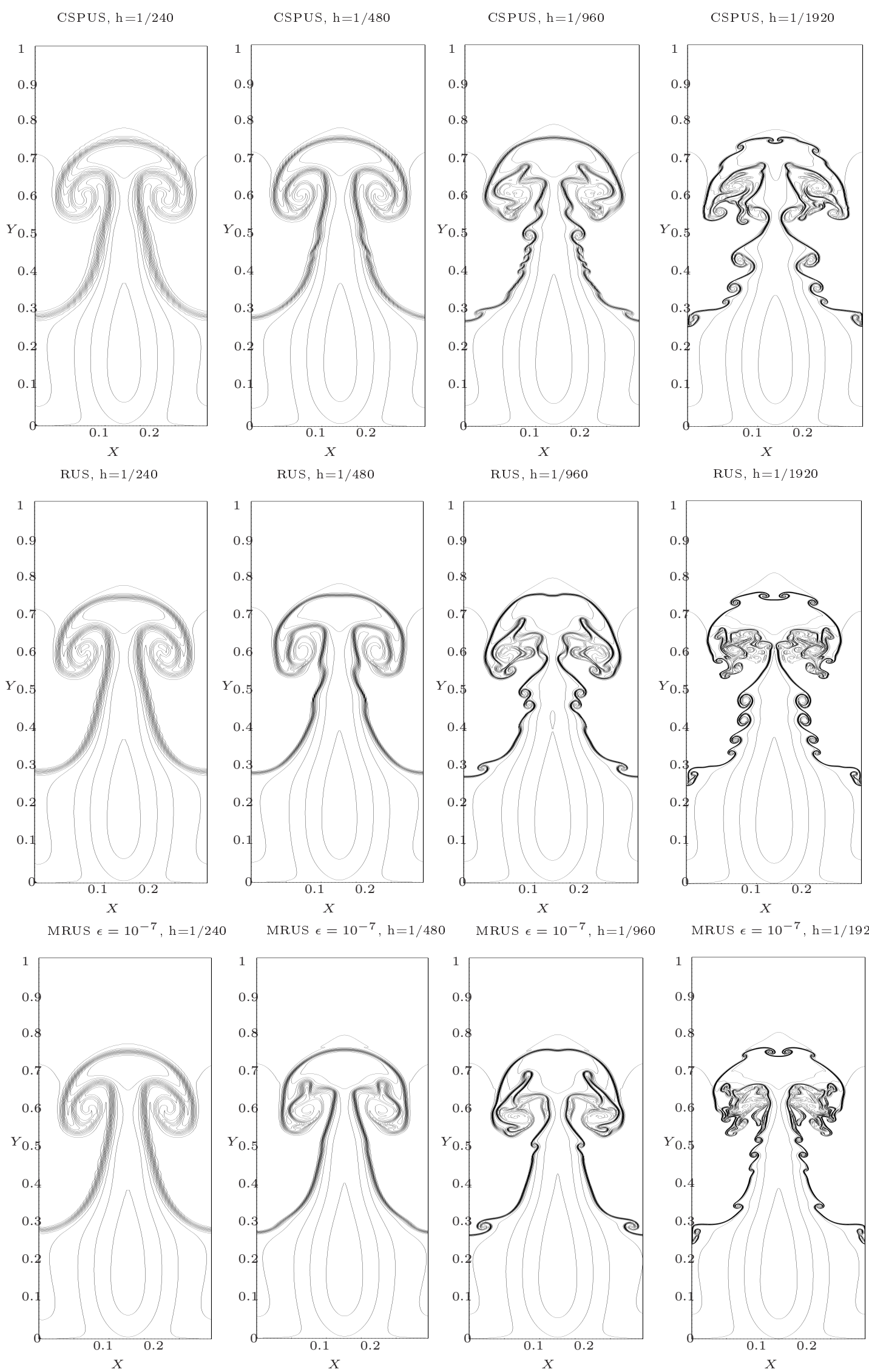

Figura 7.16: Contornos da densidade para o problema das instabilidades de Raylegh-Taylor. Linhas de contorno vão de $\rho=0.952269$ até $\rho=2.14589$. 


\subsection{Sistema não-linear de águas rasas}

Nesta seção são apresentados resultados numéricos obtidos com o uso dos esquemas MC, van Albada, FDHERPUS, RUS, CSPUS e MRUS (o esquema MC foi tomado como referência). O problema escolhido foi o da ruptura de uma barragem radial (ver por exemplo LeVeque (2004) [41]), modelado pelas equações de águas rasas (2.23) com termo fonte $S=S(u, h) ; h$ e $u$ sendo o nível do fluido (altura) e $u$ a velocidade, respectivamente.

O problema consiste em duas porções do mesmo fluido sujeitas à ação da gravidade (ver Figura 7.17 - caso (a)); após iniciar o escoamento, pela ruptura de um filme envolvendo a barragem circular, ambas as porções de fluidos iniciam seus movimentos dando origem a duas ondas de choque simétricas: uma propagando-se radialmente para fora e uma outra de rarefação propogando-se para dentro. O fenômeno que aparece no escoamento é amplamente utilizado para a avaliação comparativa de diferentes técnicas numéricas para fluxos interfaciais e problemas de impacto. O objetivo é, além de verificar se os esquemas propostos são capazes de simular esse fenômeno, investigar a capacidade de captura do choque-simétrico. Nos cálculos foi considerado o método de Godunov de primeira ordem com termo de correção e os limitadores de fluxo correspondentes aos esquemas van Albada, FDHERPUS, RUS, CSPUS e MRUS.

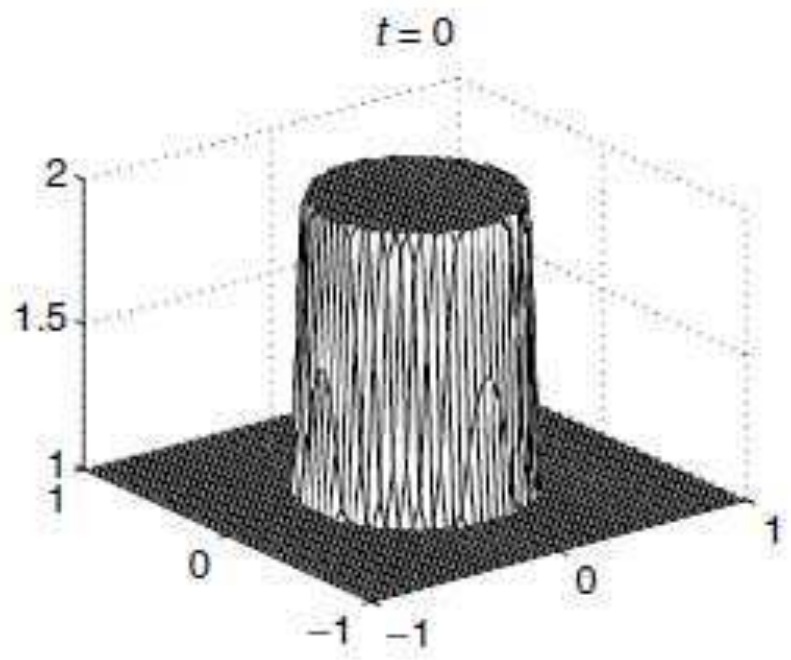

(a)

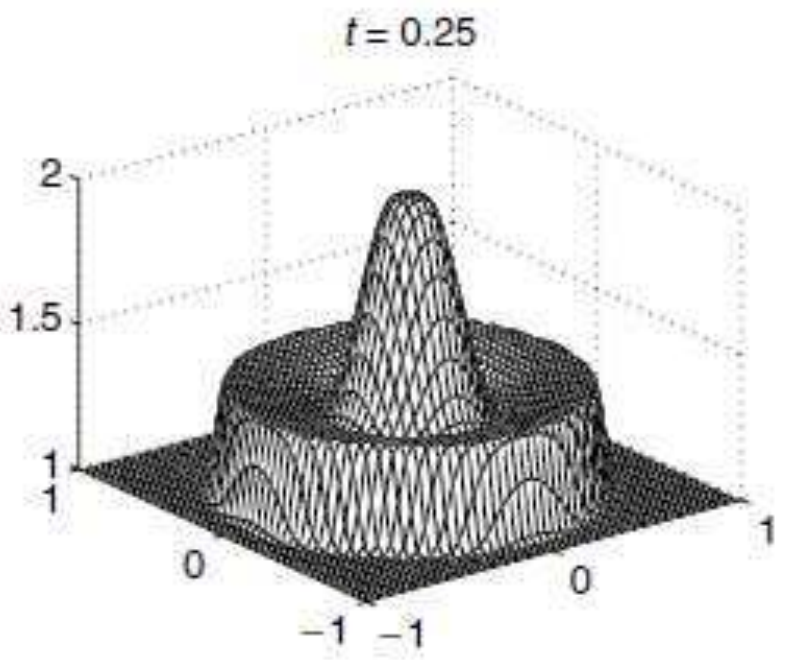

(b)

Figura 7.17: Profundidade da água $h$ para o problema de ruptura de uma barragem radial nos tempos $t=0$ e $t=0.25$. 
Para a simulação deste fenômeno foi considerada uma barragem circular de raio de 0.5 , localizada no centro de um domínio computacional de dimensões $[0,2.5] \times[0,2.5]$. O nível da barragem circular é inicialmente $h=2$ e está apoiada em outra barragem com altura $h=1$ (ver Figura 7.17). Os seguintes dados foram utilizados nas simulações usando os esquemas van Albada, FDHERPUS, RUS, CSPUS e MRUS: malha com $125 \times 125$ células computacionais, número de Courant $C=0.9$ e tempo final de simulação $t=1.5$. A solução de referência, usando o esquema MC, foi gerada numa malha de $2000 \times 2000$ células computacionais.

A evolução da altura $h$, obtida com o esquema FDHERPUS, está mostrada na Figura 7.18. As alturas obtidas com os outros esquemas não são apresentadas aqui, pois apresentaram resultados bem similares aos obtidos com o esquema FDHERPUS; a Figura 7.19, mostra uma vista de topo do contorno de $h$ obtido com os esquemas MC, van Albada, FDHERPUS, RUS, CSPUS e MRUS com $\epsilon=10^{-7}$. Para uma análise mais quantitativa, está apresentado na Figura 7.20 o perfil global de $h$ ao longo da reta $y=0$ obtido com todos os esquemas; a Figura 7.21 corresponde às ampliações destacadas na Figura 7.20. Pode-se observar por essas Figuras que o método numérico equipado com os esquemas upwind propostos neste trabalho simulou com sucesso (preservando bem a simetria radial) a ruptura de uma barragem. Observou-se também que os resultados das simulações concordam muito bem com os resultados apresentados na referência [41].

Os limitadores correspondentes aos esquemas FDHERPUS e MRUS com parâmetro $\epsilon=10^{-7}$ forneceram os melhores resultados e o que mostrou o pior resultado foi o limitador correspondente ao esquema CSPUS. O esquema de van Albada, como nas outras simulações, permaneceu (praticamente) em quarto lugar na classificação geral. 

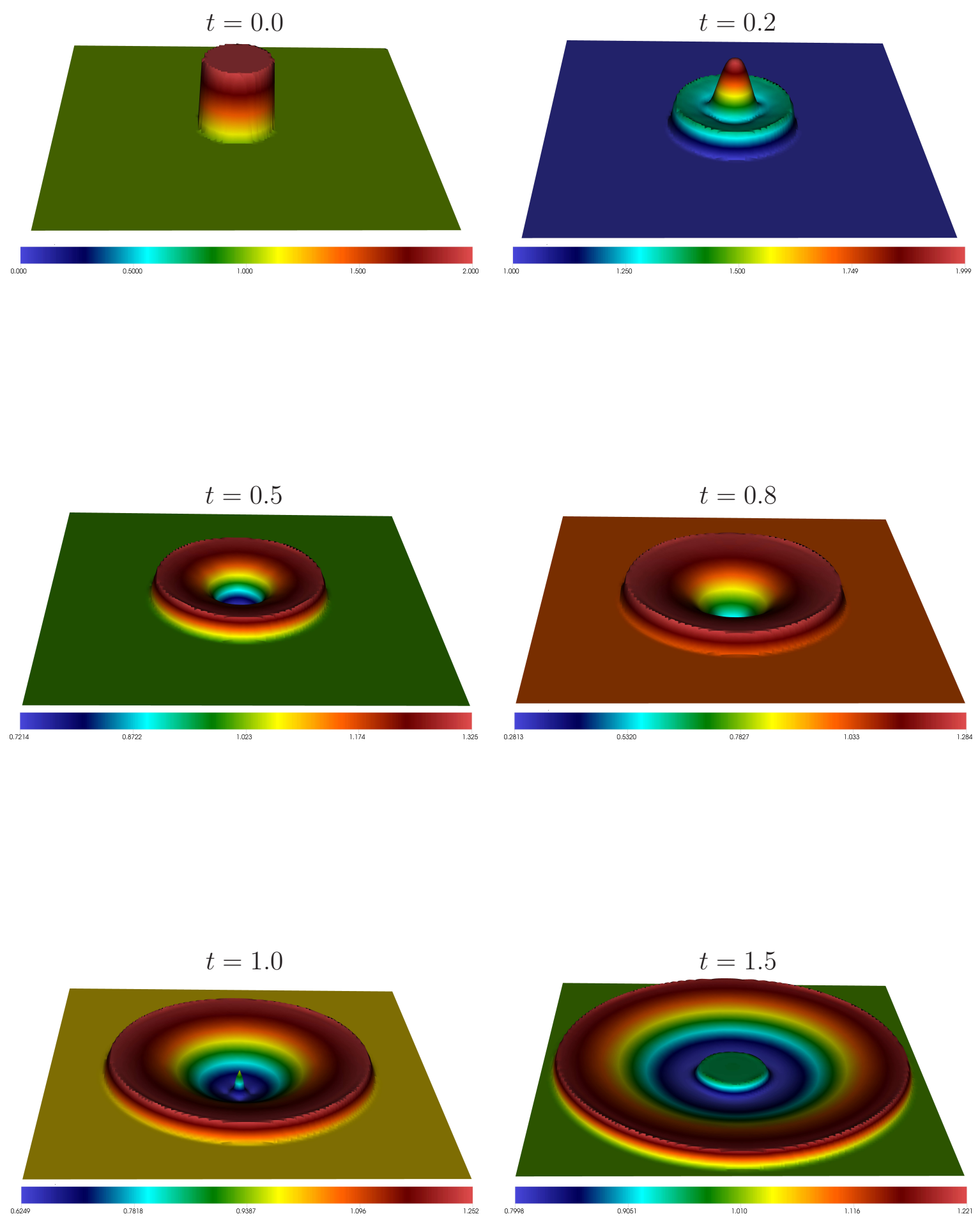

Figura 7.18: Evolução do problema de ruptura de uma barragem radial para o esquema FDHERPUS. 

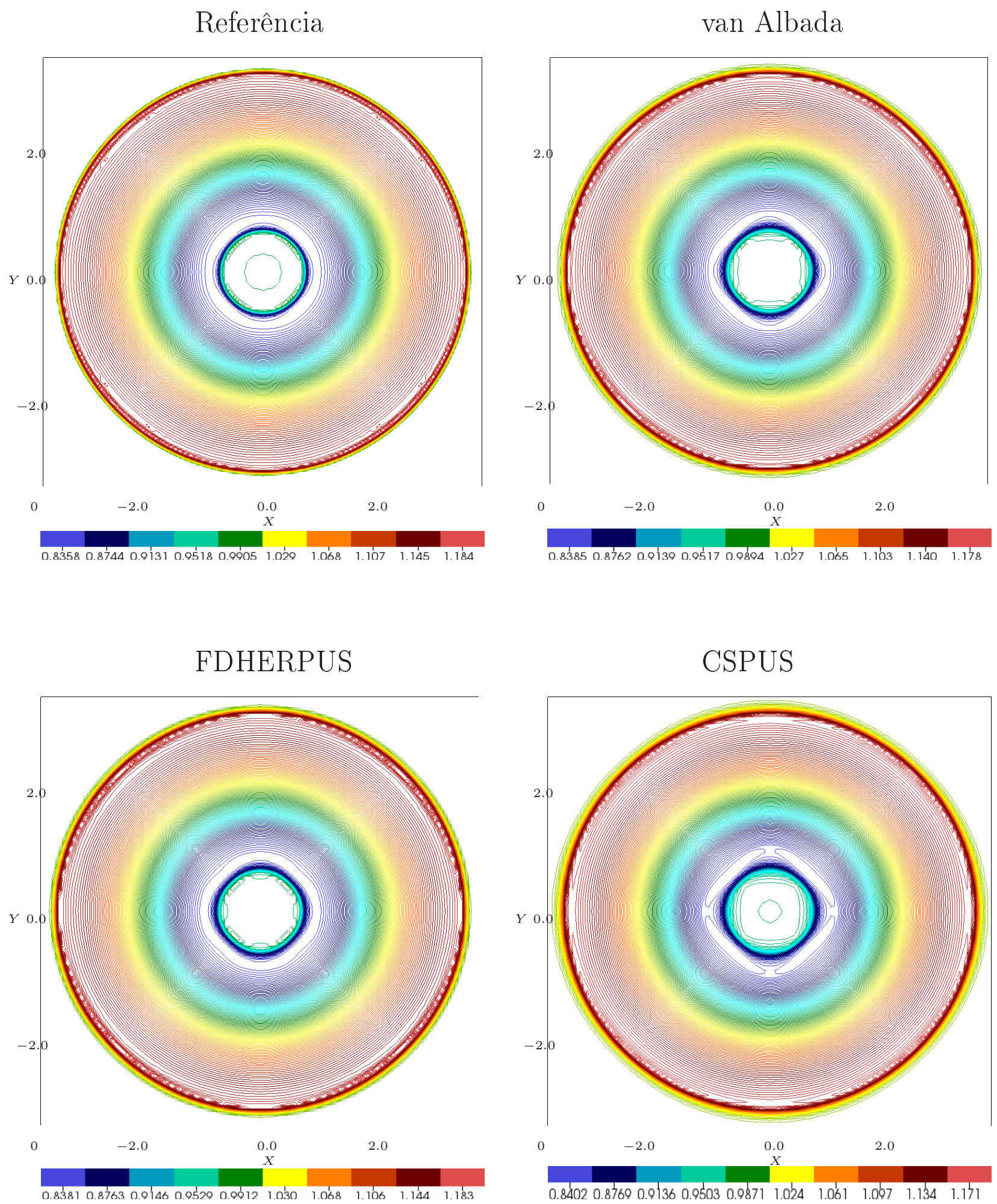


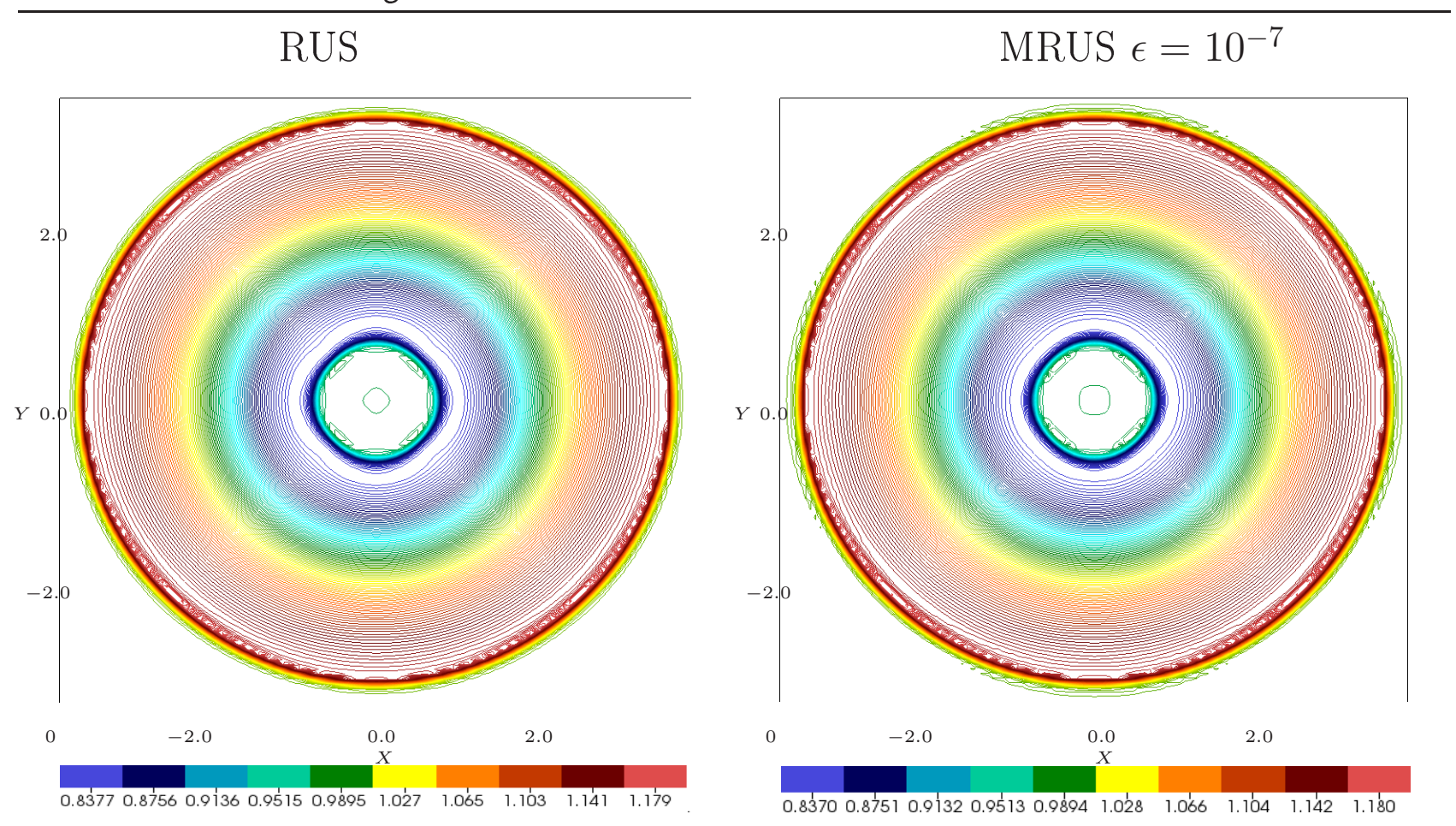

Figura 7.19: Altura $h$ para o problema de ruptura de uma barragem radial no plano $x \perp y$.

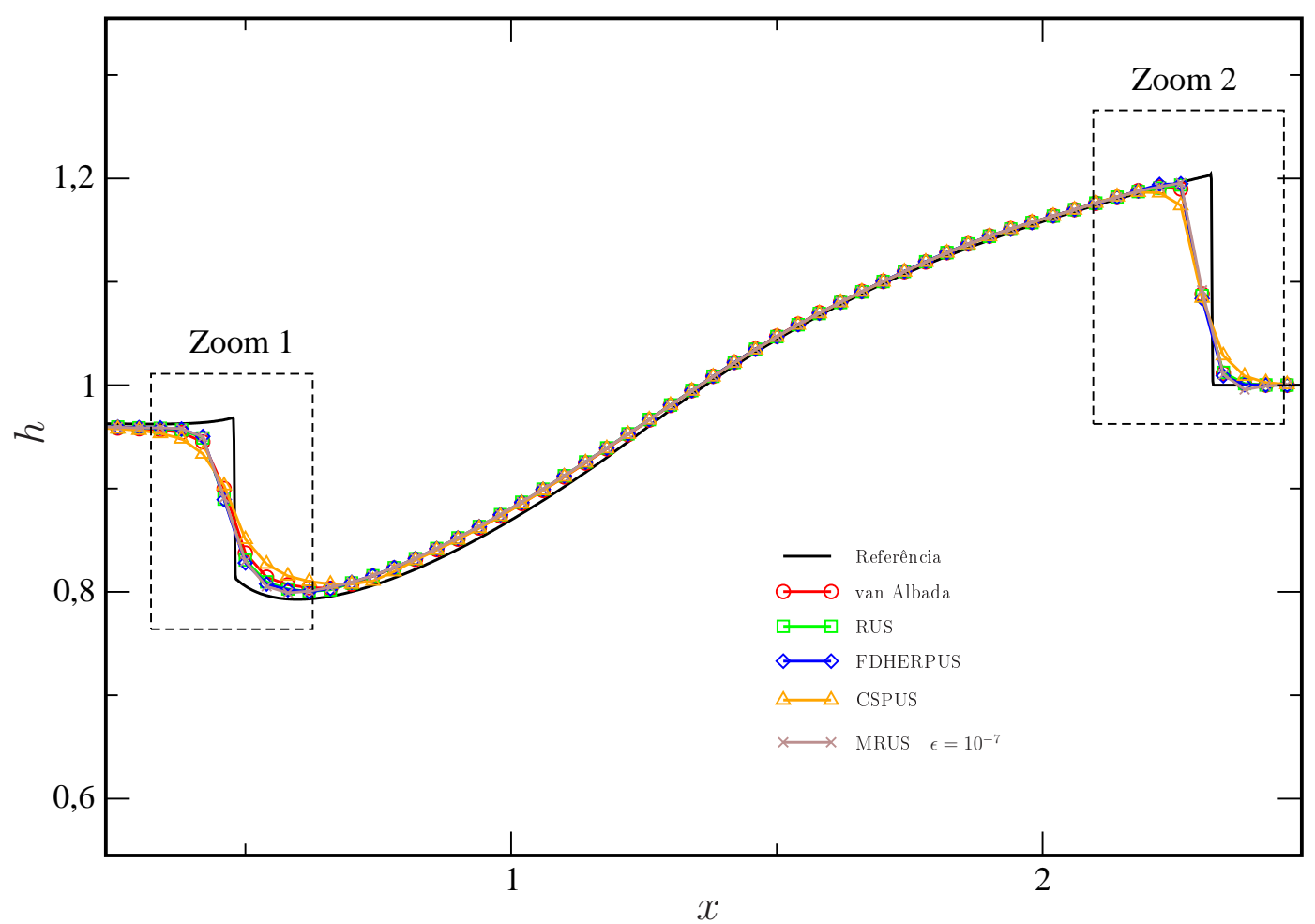

Figura 7.20: Comparação dos resultados obtidos sobre a reta $y=0$ para o problema ruptura de uma barragem radial. 
Zoom 1

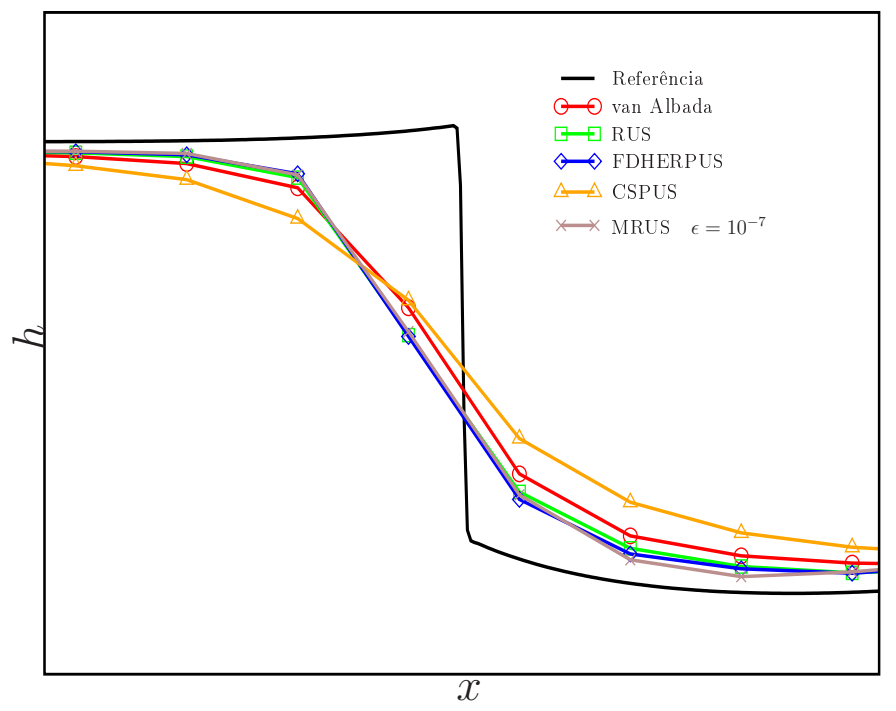

Zoom 2

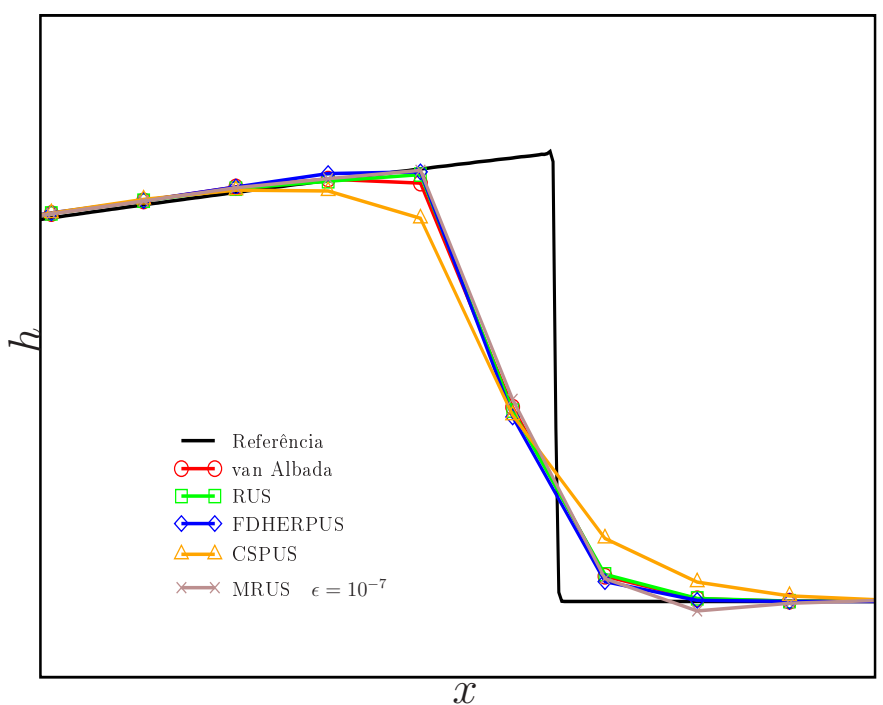

Figura 7.21: Ampliação das regiões destacadas na Figura 7.20 para o problema ruptura de uma barragem radial.

\subsection{Escoamentos incompressíveis laminares 2D e 2D-1/2}

Nesta seção seão apresentados resultados numéricos de problemas de escoamentos de fluidos newtonianos (no regime incompressível) laminares bi-dimensionais com superfícies livres móveis, governados pelas equações de Navier-Stokes (2.28). Verifica-se aqui o desempenho do esquema FDHERPUS, uma vez que ele se destacou nas simulações de leis de conservação hiperbólicas. O ambiente de simulação Freeflow Castelo (2000) [10] foi adotado nos cálculos. Quatro problemas foram simulados: um jato livre sobre uma superfície rígida impermeável (ver por exemplo Watson (1964) [82]), o colapso de uma coluna de fluido (ver por exemplo Colagrossi (2003) [12]) e o experimento de Taylor (Taylor (1974) [68]) e ressalto hidraúlico (ver por exemplo Kurihara (1946) [35]).

\subsubsection{Jato livre 2D sobre uma superfície rígida impermeável}

Nesta simulação, considerou-se um jato livre que incide perpendicularmente sobre uma parede rígida impermeável, sob ação da gravidade. A Figura 7.22 ilustra a geometria utilizada nos cálculos. Para validar o método numérico equipado com o esquema FDHERPUS, foi considerado como solução referência a solução analítica para a altura $H$ proposta por Watson (1964) [82] dada por

$$
H(x)= \begin{cases}\frac{\pi}{\sqrt{3}} \frac{\nu(x+l)}{Q}, & x \geq x_{0} \\ r_{i}+\left(\frac{1-2 \pi}{3 \sqrt{3 c^{2}}}\right) \delta(x), & x<x_{0},\end{cases}
$$

em que $Q=r_{i} U_{0}$ é a vazão e $r_{i}=L_{0} / 2$ é o raio do injetor. Os parâmetros $x_{0}, l$ e $\delta$ são calculados como: 


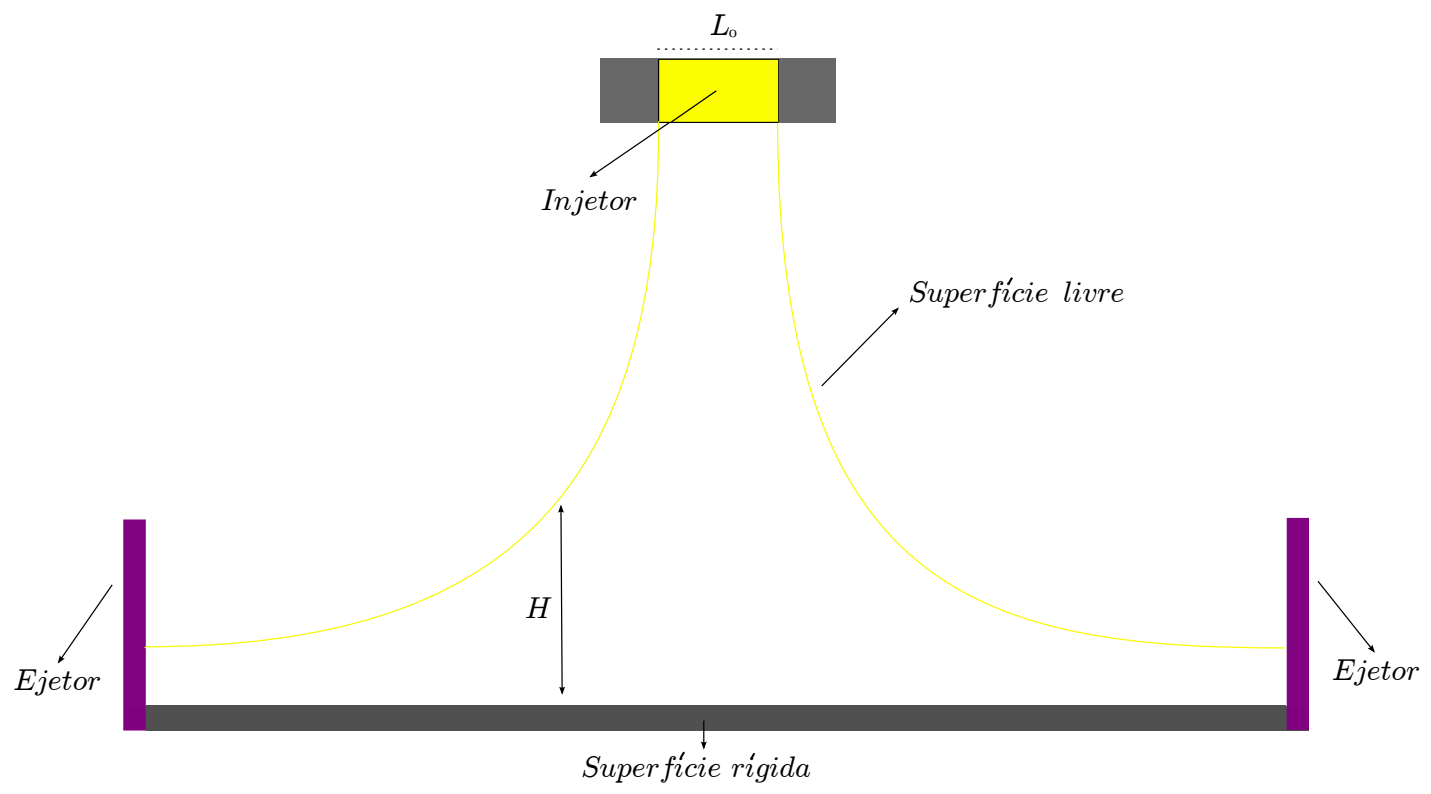

Figura 7.22: Ilustração esquemática para o problema de um jato livre sobre uma superfície rígida impermeável.

$$
\begin{gathered}
x_{0}=\left(\frac{3 \sqrt{3} c(\pi-c) \sqrt{3}}{2 \pi^{2}}\right) r_{i} R e, \\
l=\left(\frac{3 \sqrt{3} c(2 \sqrt{3} c-\pi)}{2 \pi^{2}}\right) r_{i} R e, \\
\delta^{2}(x)=\frac{3 \sqrt{3} c^{3}}{2(\pi-c \sqrt{3})} \frac{\nu x}{U_{0}}
\end{gathered}
$$

com constante $c=1.402$, e $U_{0}$ sendo a velocidade de injeção. Para a simulação foi adotada a condição no-slip no superfície rígida; e os seguintes dados foram utilizados:

- Malha I: $100 \times 25$ células computacionais;

- Malha II: $200 \times 50$ células computacionais;

- Malha III:400 × 100 células computacionais;

- Malha IV: $800 \times 200$ células computacionais;

- Dimensão do domínio: $0.1 m \times 0.002 m$;

- Raio do injetor: $r_{i}=0.002 m$;

- Escala de comprimento: $L_{0}=2 r_{i}=0.004 m$;

- Escala de velocidade: $U_{0}=1 \mathrm{~m} / \mathrm{s}$;

- Coeficiente de viscosidade: $\nu=2 \times 10^{-6} \mathrm{~m}^{2} / \mathrm{s}$; 
- Número de Reynolds: $R e=2000$;

As Figuras 7.23, 7.24 e 7.25 mostram os componentes de velocidade ( $u$ e $v$ ) e a pressão $p$, respectivamente, calculados numa malha com $800 \times 200$ células computacionais. Em particular, nesta Figura, vê-se a simulação da superfície livre do fluido com o esquema FDHERPUS no tempo $t=0.1 s$. Uma análise mais quantitativa está apresentada na Figura 7.26, onde contém uma comparação das soluções obtidas com o esquema FDHERPUS usando-se as malhas I, II, III e IV, mais a solução analítica de Watson (1964) [82]. Pode-se observar que, para malhas mais refinadas, a altura $H$ do fluido sobre o contorno rígido obtida com o esquema FDHERPUS acompanha a solução analítica de Watson; a discrepância entre esses dados pode ser atribuída ao fato de que a solução de Watson é somente uma aproximação e também ao fato de que a solução de Watson foi obtida considerando um escoamento paralelo à superfíice rígida (sem ponto de estagnação) e o apresentado aqui é perpendicular ao contorno rígido (ver Figura 7.22) com ponto de estagnação.

\section{Contorno da velocidade na direção $x$}
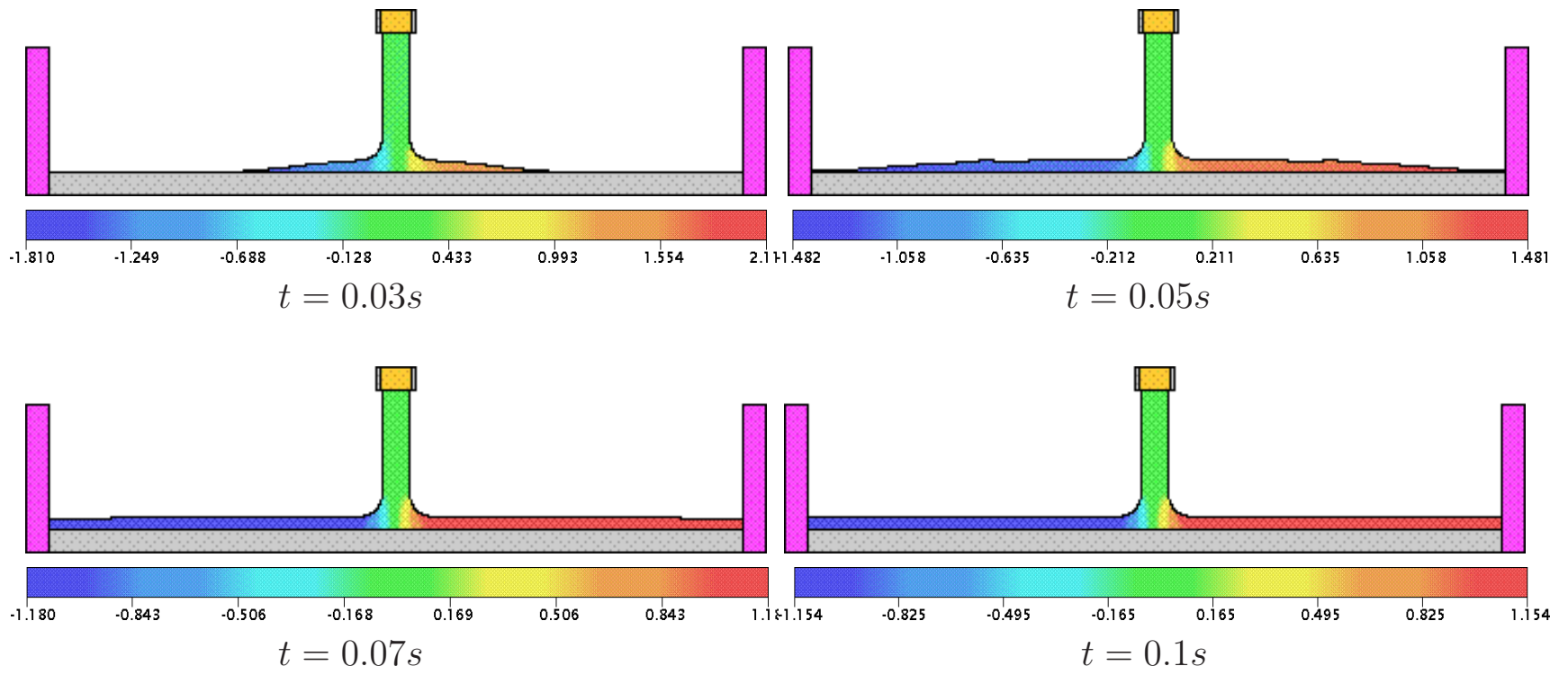

Figura 7.23: Contorno da velocidade na direção $x$ para o problema de jato livre sobre uma superfície livre impermeável.

\subsubsection{Colapso de uma coluna 2D de fluido}

O problema de colapso de uma coluna de fluido newtoniano envolvendo superfícies livres móveis (ver por exemplo Murrone e Guillard (2008) [47]) tem sido objeto de muitas investigações, tanto numéricas quanto experimentais. O fenômeno consiste em uma porção de fluido inicialmente em repouso sob a ação da gravidade que se espalha sobre uma superfície rígida impermeável, como ilustrado na Figura 7.27. O problema foi estudado experimentalmente 


\section{Contorno da velocidade na direção $y$}
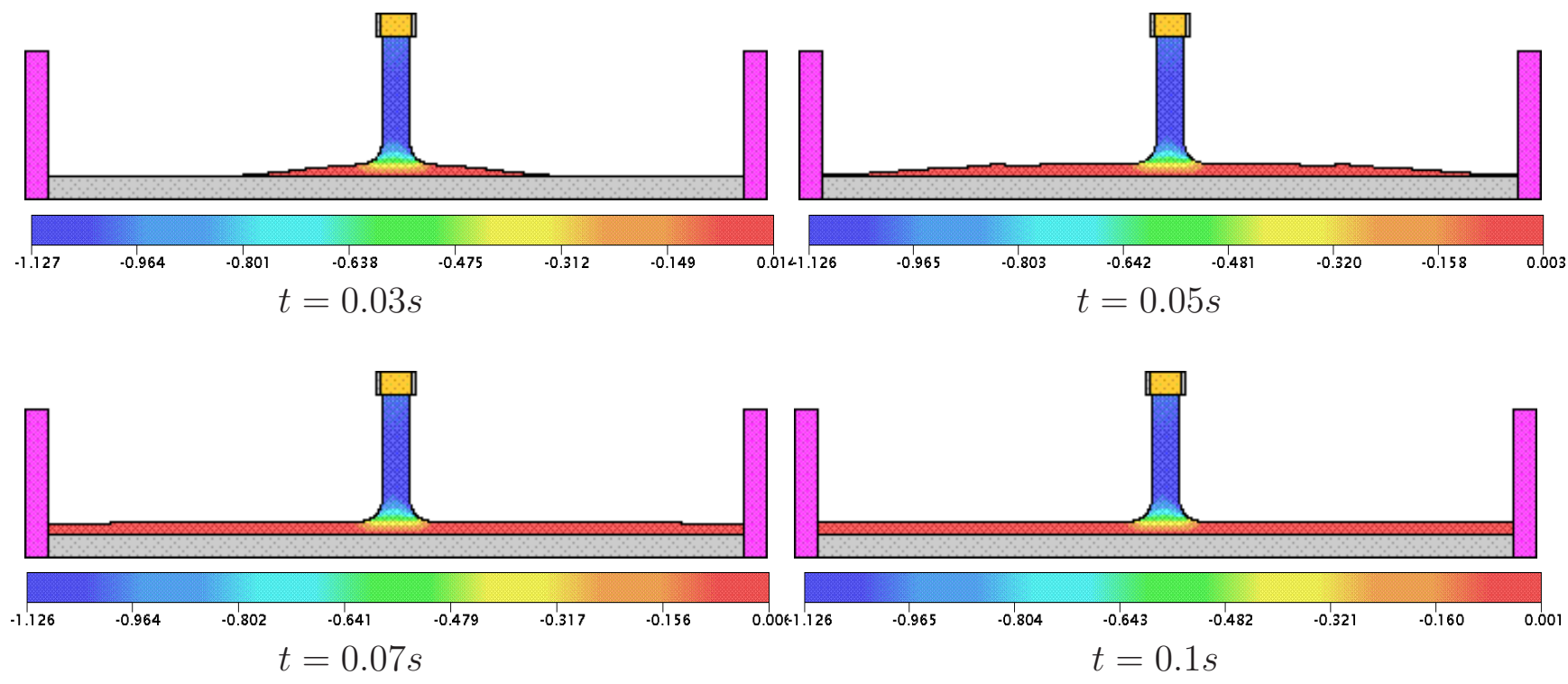

Figura 7.24: Contorno da velocidade na direção y para o problema de jato livre sobre uma superfície livre impermeável.

\section{Contorno da pressão}
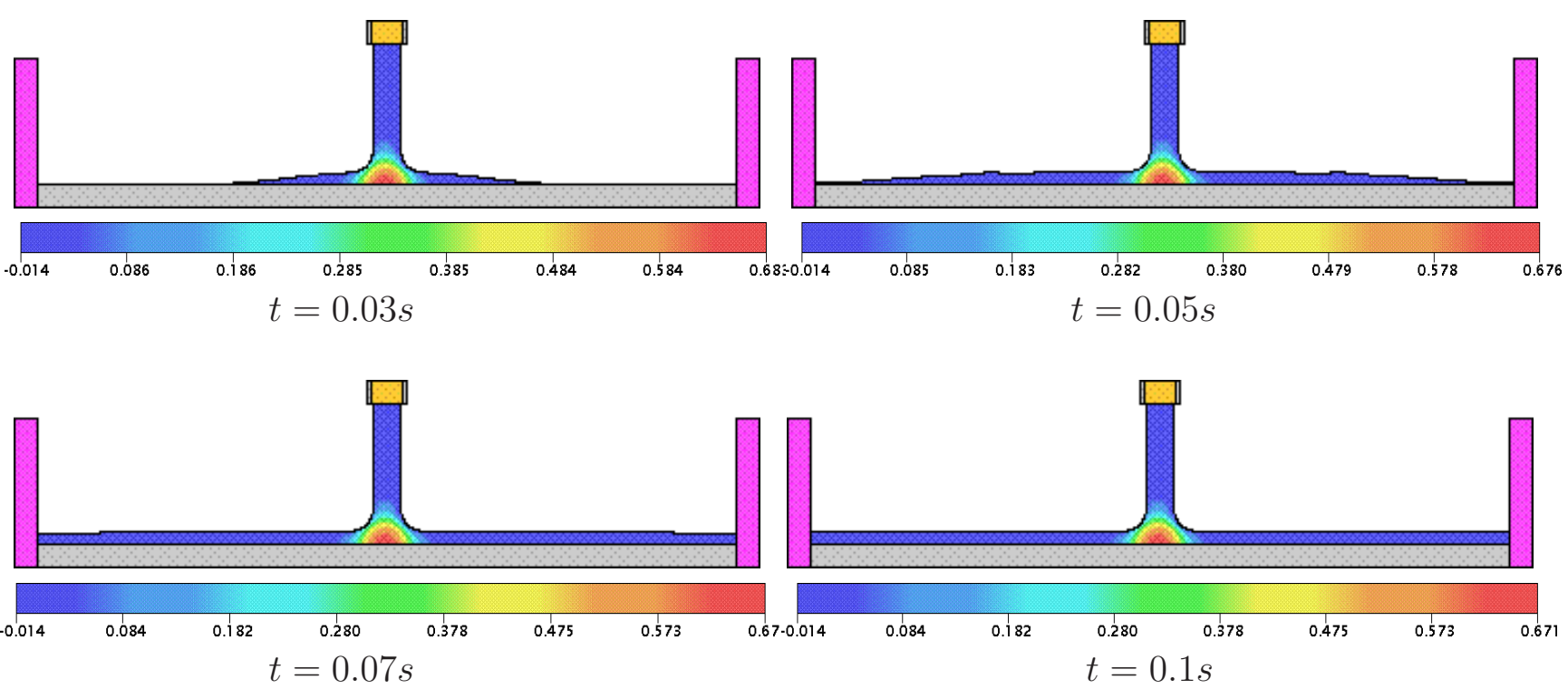

Figura 7.25: Contorno da pressão para o problema de jato livre sobre uma superfície livre impermeável. 


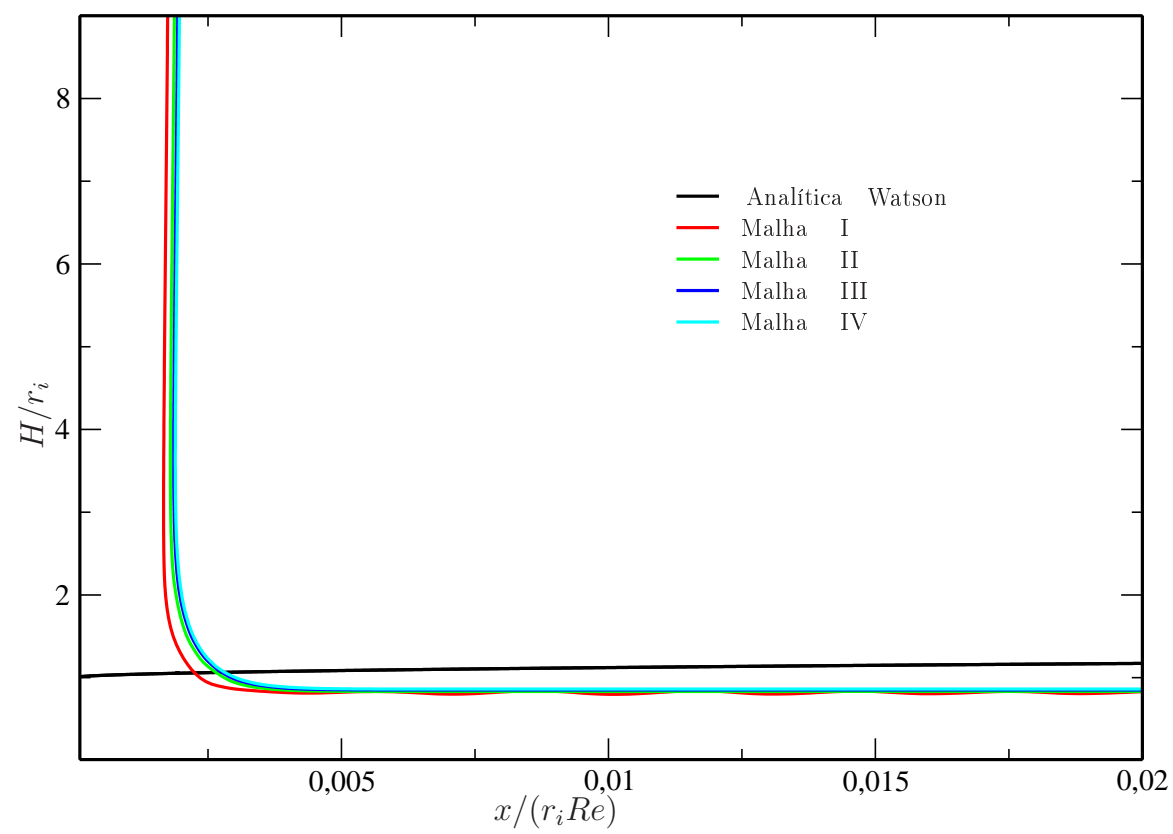

Figura 7.26: Comparação entre as soluções numéricas com o esquema FDHERPUS e a solução analítica de Watson (1964) [82] para a altura $H$.

pela primeira vez por Martin e Moice (1952) [46], e teoricamente por Ritter (1982) [57]; tem sido resolvido também via métodos numéricos por vários autores, dentre eles pode-se citar a metodologia de Colagrossi e Landrini (2003) [12], onde seus resultados foram confrontados com os dados gerados pelos métodos SPH (Smoothed Particle Hydrodinamics method) de Colagrossi e Landrini (2003) [12], BEM (Bondary Element Method) de Greco (2003) [25], e Level Set de Colicchio et al. (2003) [13]. Para a simulação numérica do problema foi considerada a condição de contorno free - slip mais os seguintes dados

- Malha: $1000 \times 200$ células computacionais;

- Dimensão do domínio: $0.5 m \times 0.1 m$;

- Dimensão da coluna de fluido: $a=0.057 m$

- Escala de comprimento: $L_{0}=a=0.057 m$;

- Escala de velocidade: $U_{0}=\sqrt{g L_{0}}=0.74778 \mathrm{~m} / \mathrm{s}$;

- Coeficiente de viscosidade: $\nu=10^{-6} \mathrm{~m}^{2} / \mathrm{s}$;

- Número de Reynolds: $R e=42623.27$;

Nas Figuras 7.28, 7.29 e 7.30 está mostrada a evolução no tempo da superfície livre e os contornos para o campo de pressão $p$ e velocidades nas direções $x$ e $y$ obtidos com o código Freeflow adaptado com o esquema FDHERPUS. Observa-se que o problema foi simulado com o uso do esquema FDHERPUS sem instabilidades na superfície livre móvel. 


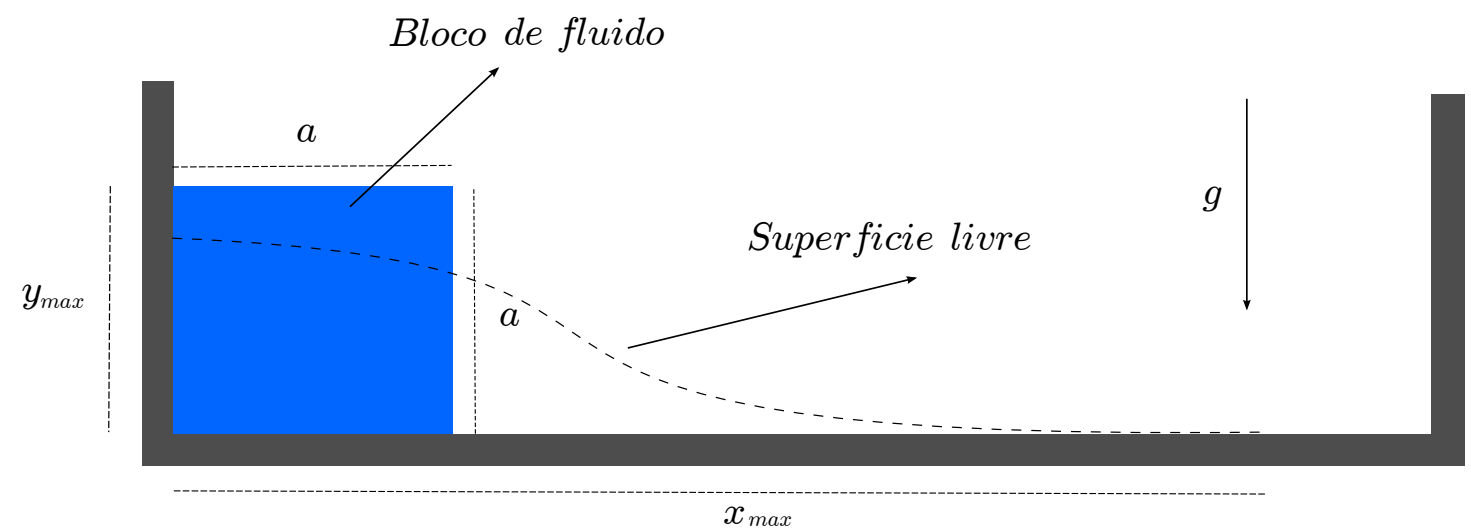

Figura 7.27: Representação esquemática para o problema do colapso de uma coluna de fluido.

\section{Campo de pressão}
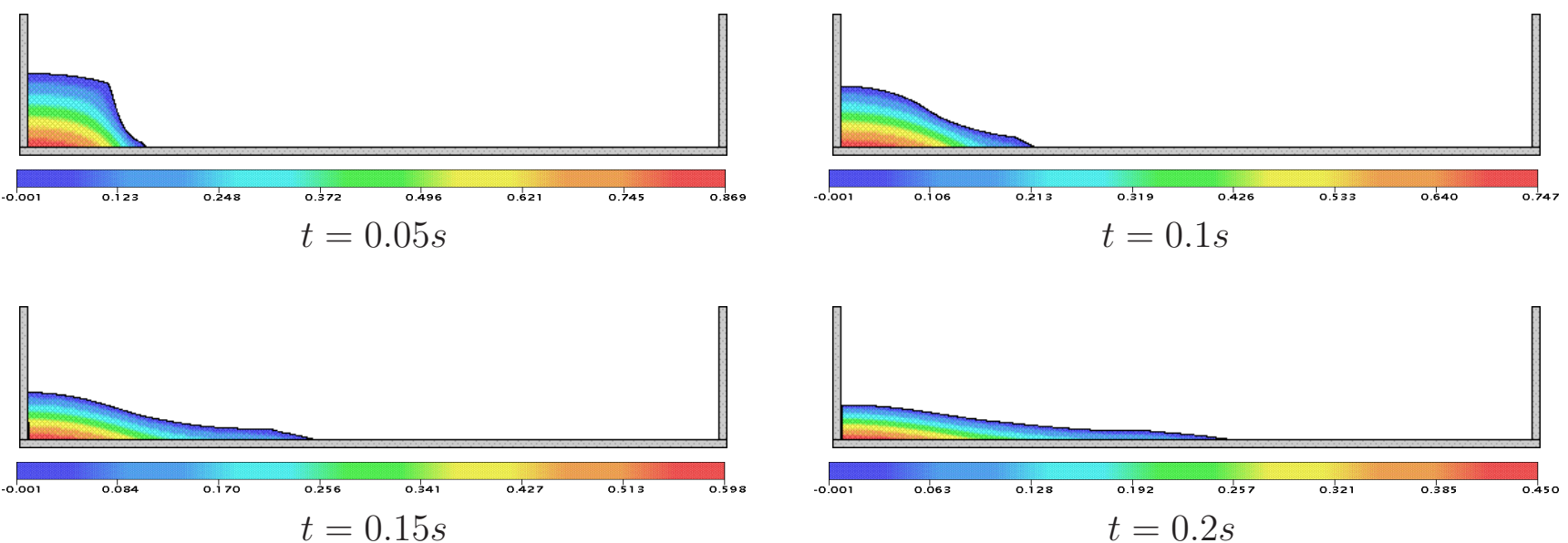

Figura 7.28: Contorno da pressão para o problema do colapso de uma coluna de fluido. FDHERPUS 


\section{Campo de velocidade em $x$}
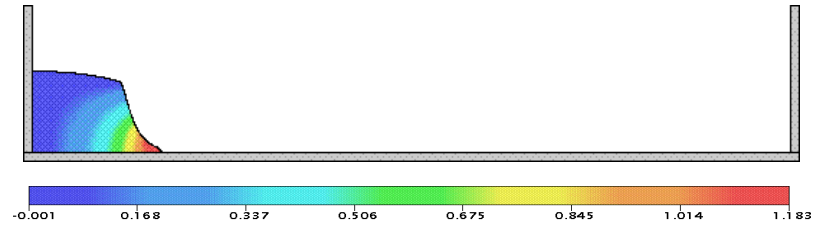

$t=0.05 s$
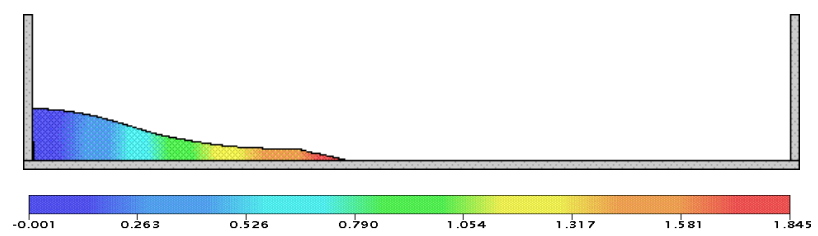

$t=0.15 s$
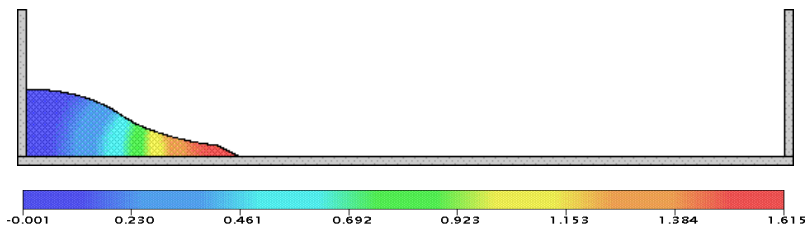

$t=0.1 s$

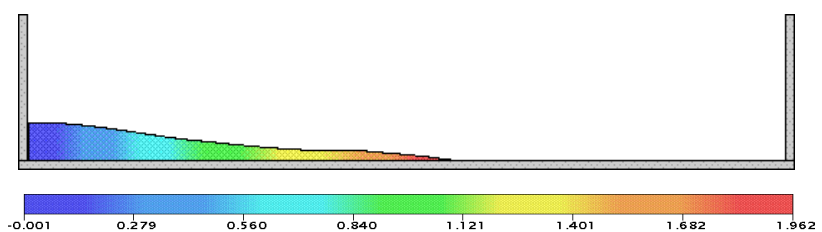

$t=0.2 s$

Figura 7.29: Contorno da velocidade em $x$ para o problema do colapso de uma coluna de fluido.

\section{Campo de velocidade em $y$}
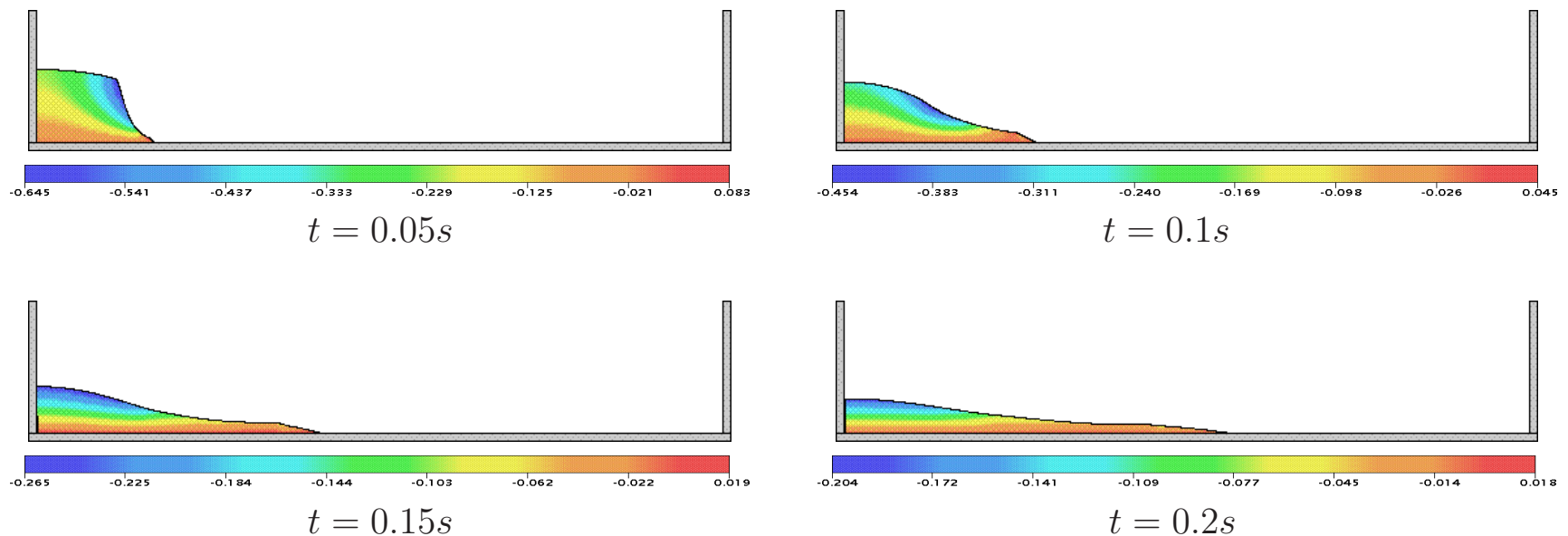

Figura 7.30: Contorno da velocidade em y para o problema do colapso de uma coluna de fluido.

Para avaliar o desempenho do esquema FDHERPUS, foi feita uma comparação dos resultados numéricos para o espalhamento horizontal $\left(x_{\max }\right)$ (ver Figura 7.27 ) derivados por este esquema e os dados numéricos, teóricos e experimentais dos autores mencionados anteriormente. Essa comparação é mostrada na Figura 7.31, em que pode-se ver claramente uma ótima concordância com os dados numéricos da literatura. 

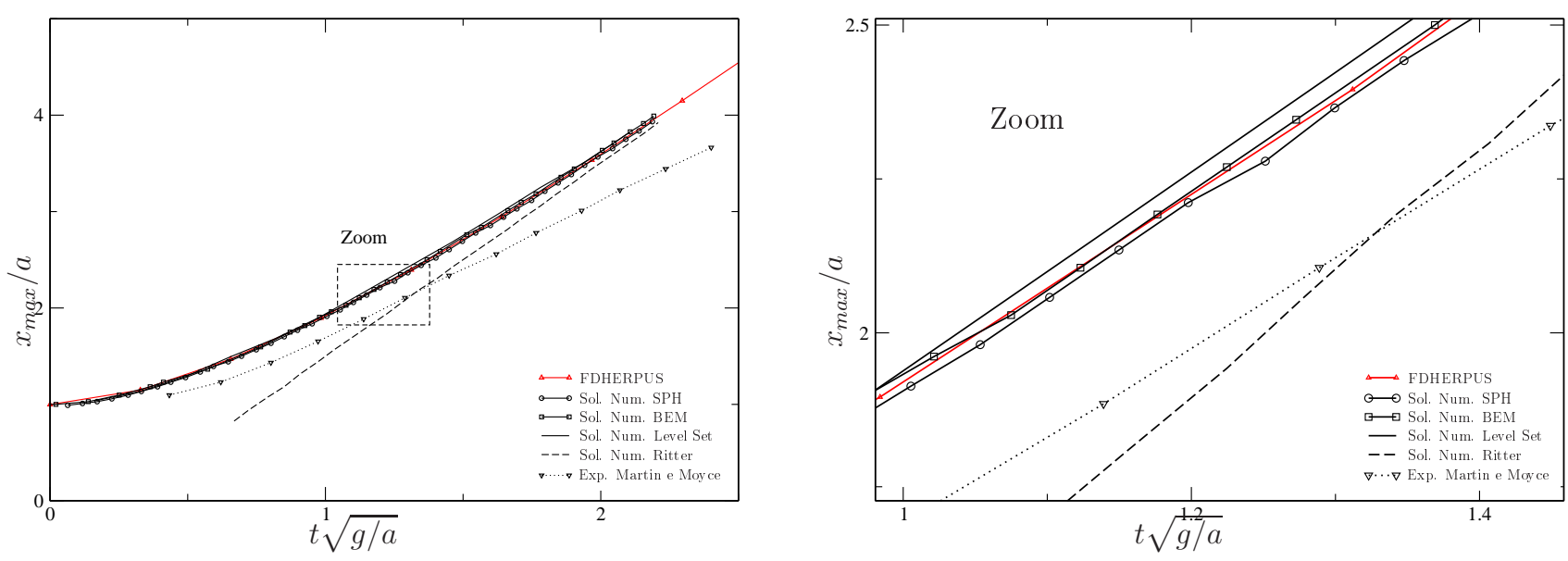

Figura 7.31: Comparação das soluções teórica, experimental e numéricas para o problema de colapso de uma coluna de fluido.

\subsubsection{Experimento de Taylor no caso $2 \mathrm{D}-1 / 2$}

O esquema FDHERPUS foi também avaliado na simulação (interessante) do experimento de Taylor (1974) [68], o qual consiste em um jato livre vertical que incide num recipiente contendo o mesmo fluido em repouso, como mostrado na Figura 7.32. O problema é útil para investigar a capacidade do esquema numérico em simular estruturas vorticais de grades escalas (estruturas coerentes) em escoamentos com superfícies livres móveis. A condição de contorno aplicada nas paredes foi no-slip. Os dados utilizados para a simulação do experimento são

- Malha: $123 \times 403$ células computacionais;

- Dimensão do domínio: $0.0615 m \times 0.2015 m$;

- Raio do injetor: $r_{i}=0.002 m$

- Altura do injetor: $h_{i}=0.03 m$

- Raio do recipiente cilíndrico: $r_{r}=0.06 \mathrm{~m}$

- Altura do recipiente cilíndrico: $h_{r}=0.17 m$

- Altura do fluido contido no recipiente: $h_{f}=0.16 m$

- Escala de comprimento: $L_{0}=2 r_{i}=0.004 m$;

- Escala de velocidade (velocidade de injeção): $U_{0}=0.5 \mathrm{~m} / \mathrm{s}$;

- Coeficiente de viscosidade: $\nu=10^{-5} \mathrm{~m}^{2} / \mathrm{s}$;

- Número de Reynolds: $R e=200$; 


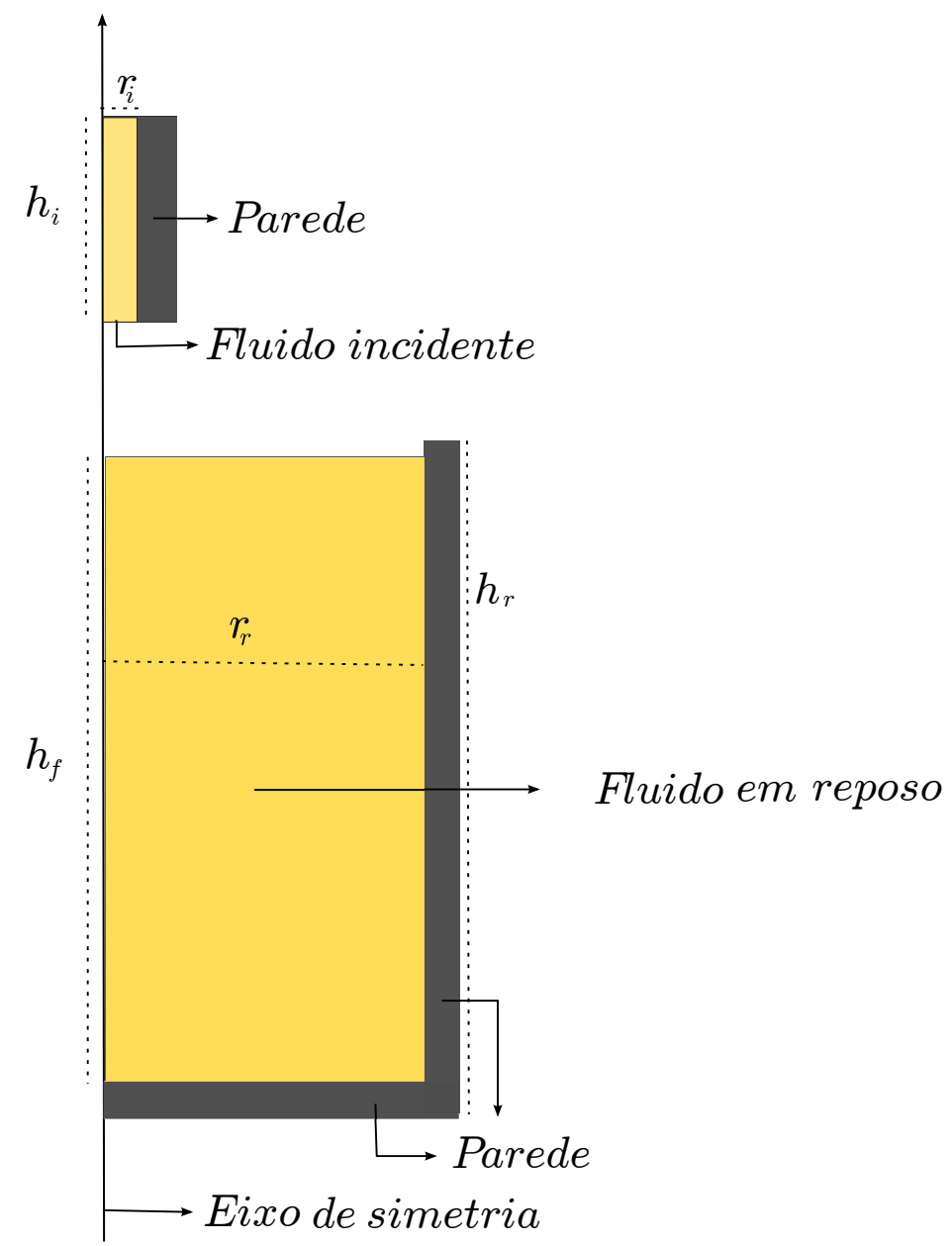

Figura 7.32: Representação esquemática para o problema de Taylor. 
Na Figura 7.33 são mostrados nos tempos $t=0.75 \mathrm{~s}$ e $t=2.5 \mathrm{~s}$ os resultados experimentais (à esquerda) e os resultados gerados pelo Freeflow (à direita) equipado com o esquema FDHERPUS. Percebe-se por essa Figura que o resultado numérico se assemelha bastante com o experimento. Em particular, a simulação no tempo $t=2.5 \mathrm{~s}$ capturou a estrutura toroidal quando houve a interação do jato penetrante com a superfície rígida. Na Figura 7.34 está mostrado a estrutura toroidal no tempo de simulação $t=10 \mathrm{~s}$, onde é possível observar a complexidade da estrutura vortical simulado com o uso do esquema FDHERPUS. Para simples ilustração, são apresentados na Figura 7.35 a interface livre que separa os dois fluidos e os contornos de pressão e velocidades nas direções $r$ e $z$.

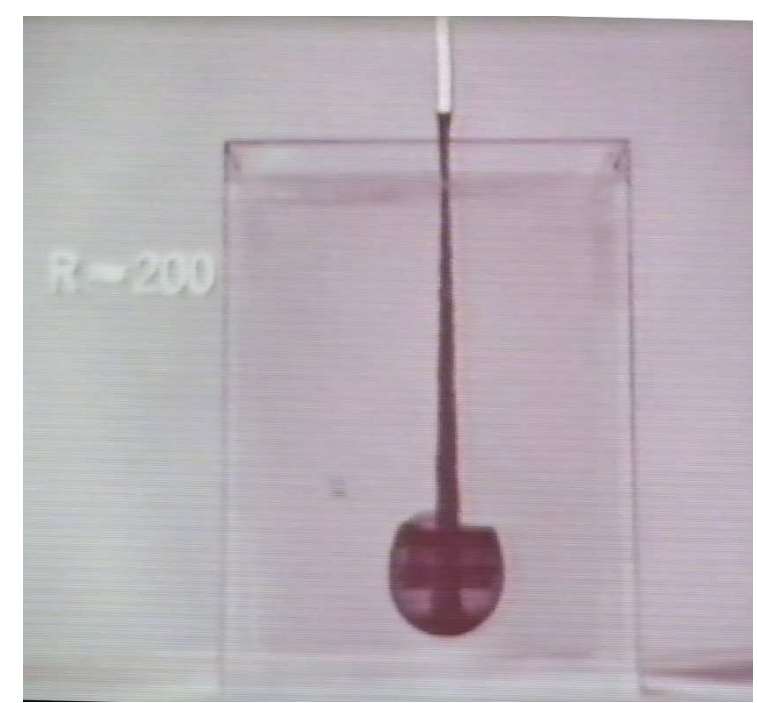

$t=0.75 s$
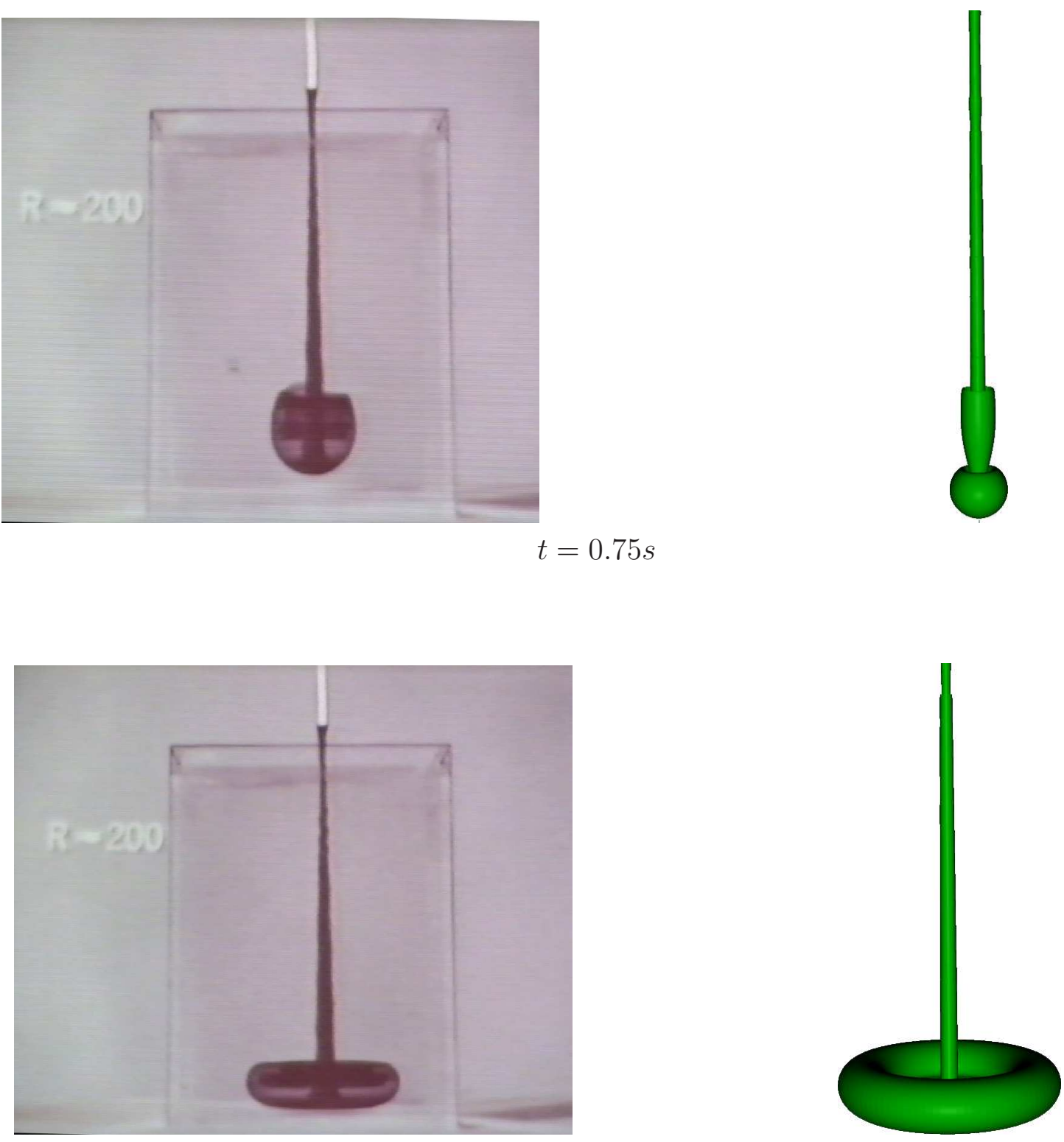

$t=2.5 \mathrm{~s}$

Figura 7.33: Resultados experimentais e numéricos para o experimento de Taylor nos tempos $t=0.75 \mathrm{~s}$ e $t=2.5 \mathrm{~s}$. O esquema FDHERPUS foi adotado nas simulações. 

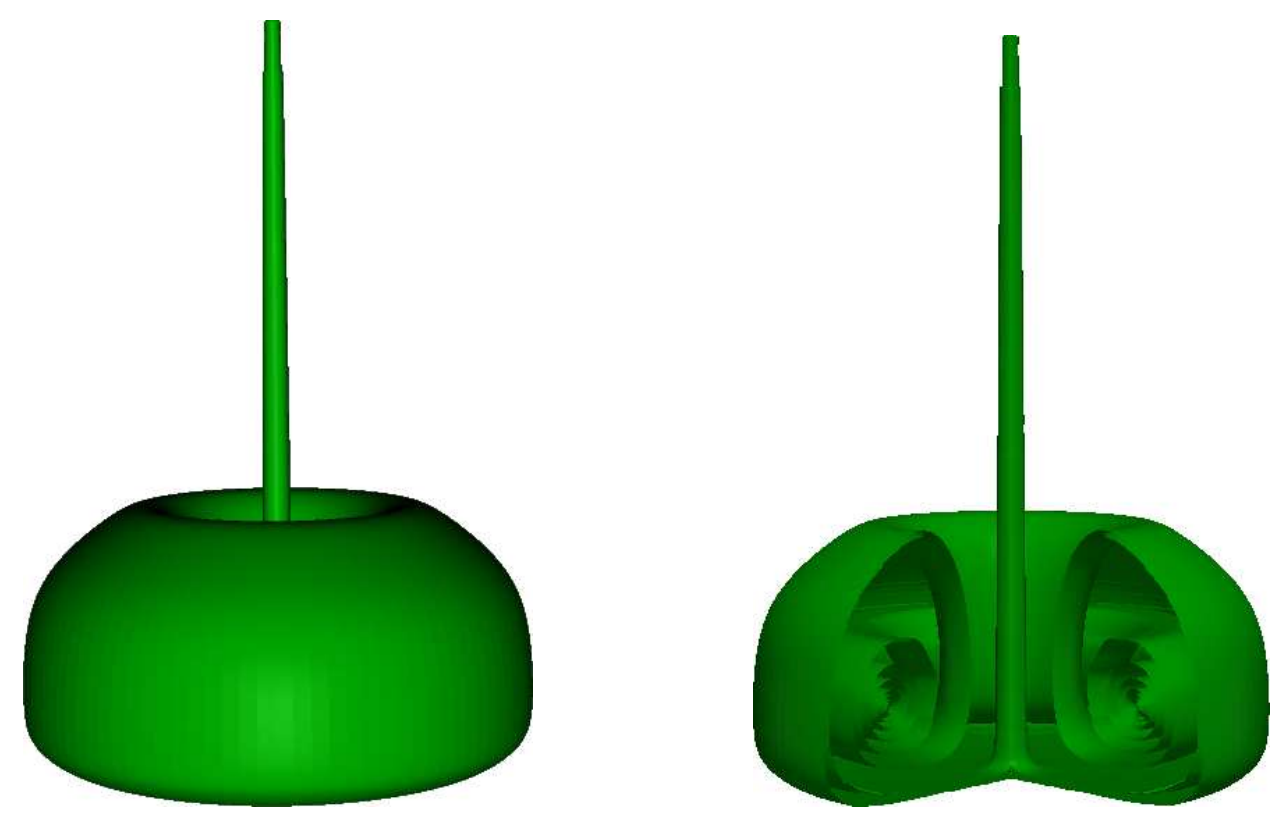

$$
t=10 s
$$

Figura 7.34: Resultado numérico para o experimento de Taylor obtido com o uso do esquema FDHERPUS no tempo $t=10.0 \mathrm{~s}$. 


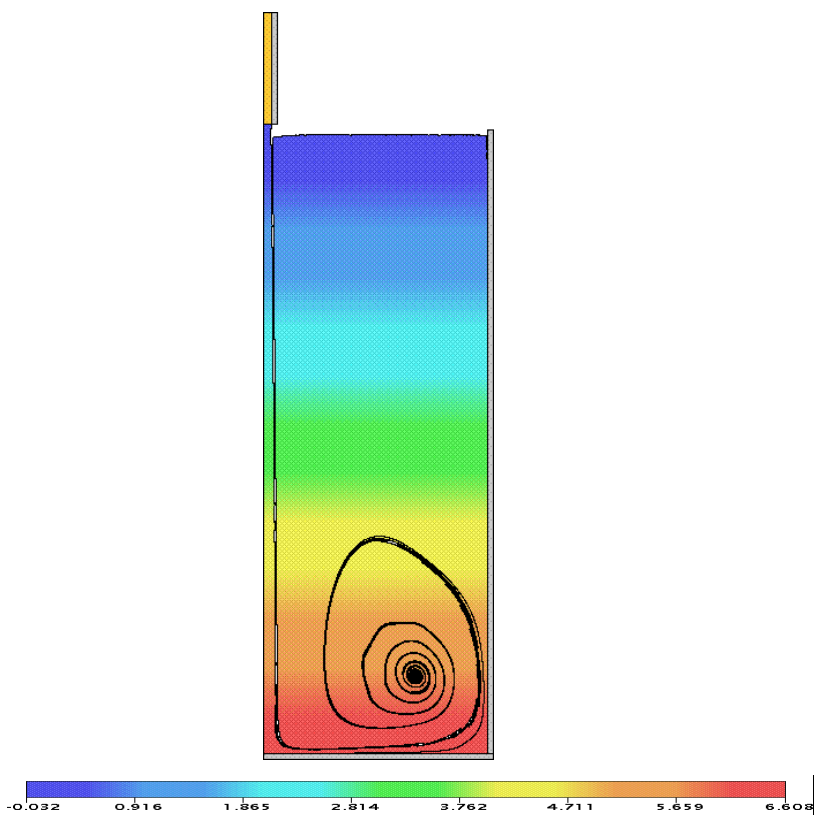

(a)

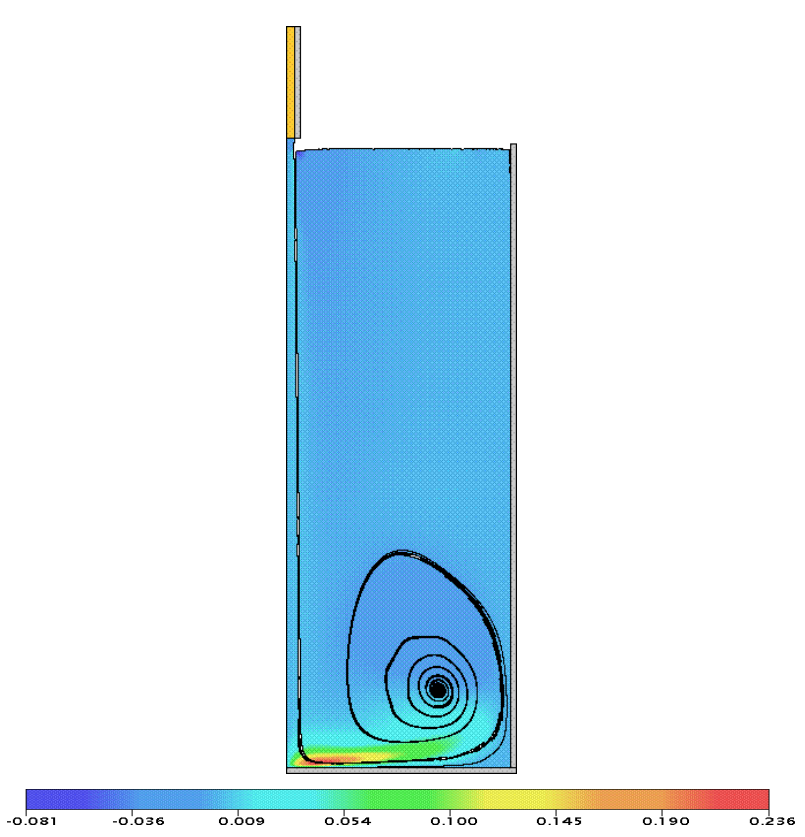

(b)

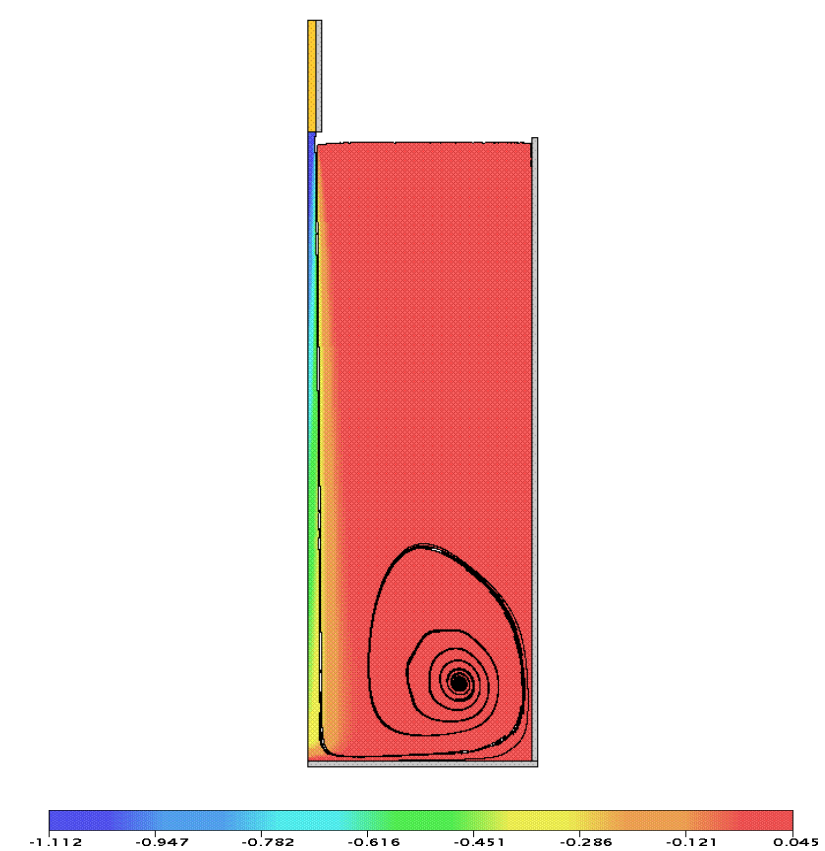

(c)

Figura 7.35: Contornos de pressão (caso (a)), velocidade na direção $x$ (caso (b)) e velocidade na direção y (caso (c)) para o experimento de Taylor obtidos com esquema FDHERPUS no tempo $t=10 \mathrm{~s}$. 


\subsubsection{Ressalto hidráulico circular no caso $2 \mathrm{D}-1 / 2$}

Ressaltos hidráulicos são problemas que aparecem com frequência na natureza e com várias aplicações tecnológicas. Eles podem ser observados facilmente nos escoamentos de líquidos em camadas finas sobre superfícies sólidas e em rios. Os movimentos da água sobre o solo, sobre as janelas ou sobre os telhados são também bons exemplos. Para o caso aqui tratado, será considerado um jato livre incidindo de maneira perpendicular sobre uma superfície rígida plana, sob ação da força de gravidade, espalhando-se de forma rápida pela superfície a partir do ponto de impacto. A geometria do problema, bem como uma ilustração da superfície livre móvel, pode ser observada na Figura 7.36.

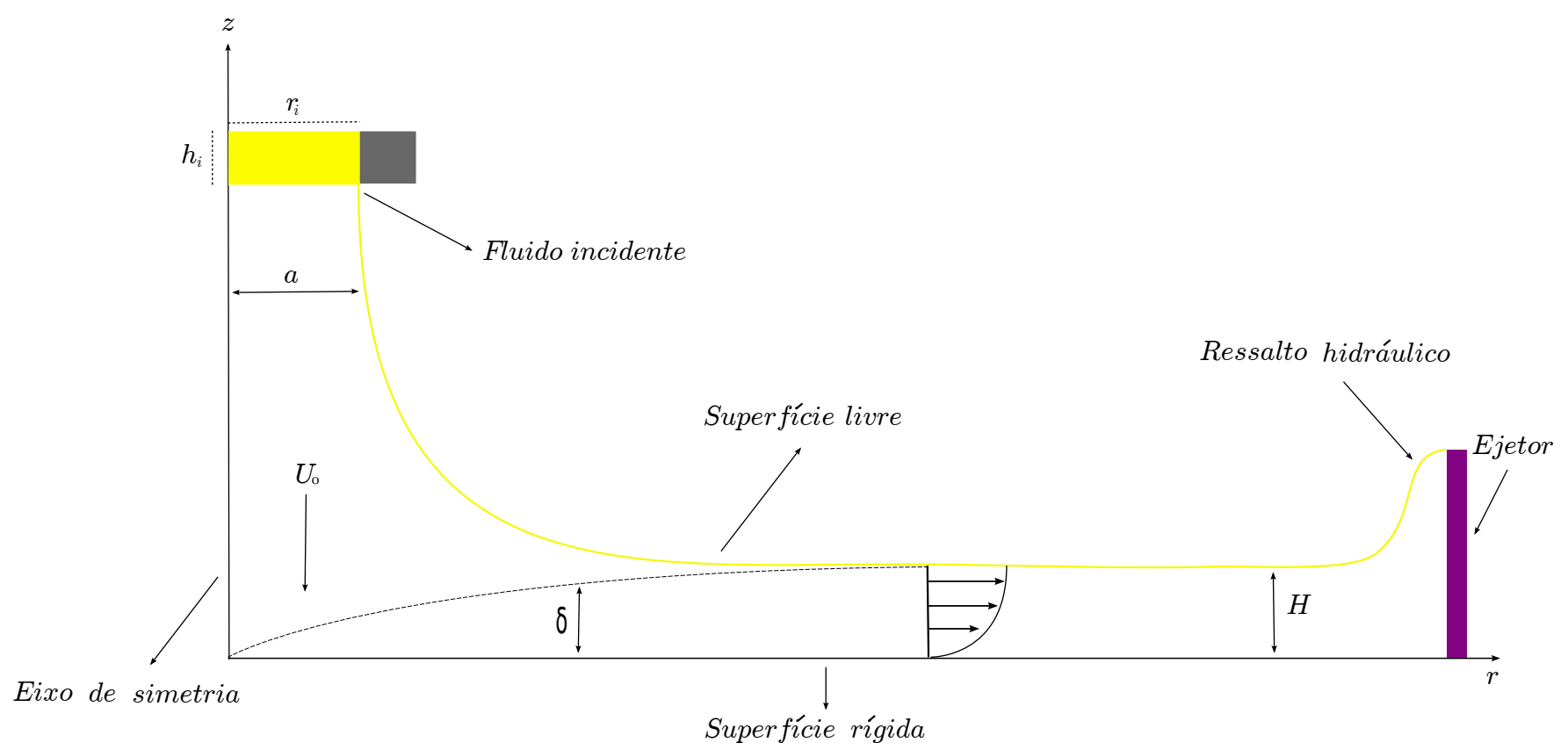

Figura 7.36: Representação esquemática para o problema de ressalto hidráulico circular.

Para este problema foi apresentada, por Watson (1964) [82] uma solução analítica para o camada de fluido de espessura $H$ (equação (7.9)); essa espessura será utilizada para verificar o desempenho do método numérico, implementado no ambiente computacional Freeflow Castelo (2000) [10] para problemas com simetria radial, equipado com o esquema FDHERPUS. É importante observar que essa solução analítica é valida somente na região longe do ponto de impacto e antes do ressalto hidráulico.

A solução analítica de Watson é dada por

$$
H(r)= \begin{cases}\frac{r_{i}^{2}}{2 r}+\left(1-\frac{2 \pi}{3 \sqrt{3} c^{2}}\right) \delta, & r<r_{0} \\ \frac{2 \pi^{2}}{3 \sqrt{3}} \frac{\nu\left(r^{3}+l^{2}\right)}{Q r}, & r \geq r_{0}\end{cases}
$$

em que, 


$$
\delta^{2}=\frac{\pi \sqrt{3} c^{3}}{\pi-c \sqrt{3}} \frac{\nu r r_{i}^{2}}{Q}
$$

onde $c=1.402, l=0.567 r_{i} R e^{\frac{1}{3}}$ é uma constante calculada com base no desenvolvimento inicial da camada limite $\delta$ e $r_{0}=0.3155 r_{i} R e^{\frac{1}{3}}$ é o raio do salto. Foi utilizada condição de contorno no-slip. Os outros dados empregados para a simulação computacional do ressalto hidráulico são descritos a seguir:

- Malha I: $400 \times 252$ células computacionais;

- Malha II: $200 \times 126$ células computacionais;

- Dimensão do domínio: $0.05 m \times 0.0315 m$;

- Raio do injetor: $r_{i}=0.004 m$

- Altura do injetor: $h_{i}=0.00075 \mathrm{~m}$

- Escala de comprimento: $L_{0}=2 r_{i}=0.008 m$;

- Escala de velocidade (velocidade de injeção): $U_{0}=0.375 \mathrm{~m} / \mathrm{s}$;

- Coeficiente de viscosidade: $\nu=1.2 \cdot 10^{-5} \mathrm{~m}^{2} / \mathrm{s}$;

- Número de Reynolds: $R e=250$;

Na Figura 7.37 apresenta-se as comparações feitas com a solução analítica de Watson para a altura $H$ e os resultados fornecidos pelo esquema FDHERPUS, para as duas malhas I e II e tempo $t=2.5 s$. Observa-se que, dentro da região de validade, as soluções obtidas com o esquema FDHERPUS estão em concordância com a solução proposta por Watson.

Para finalizar, é apresentado na Figura 7.38 uma comparação (qualitativa) tridimensional entre o experimento feito por Rai (2008) [53] e o resultado simulado com o esquema FDHERPUS. Os dados mostram claramente que o método numérico simulou com sucesso o fenômeno ressalto hidráulico. 


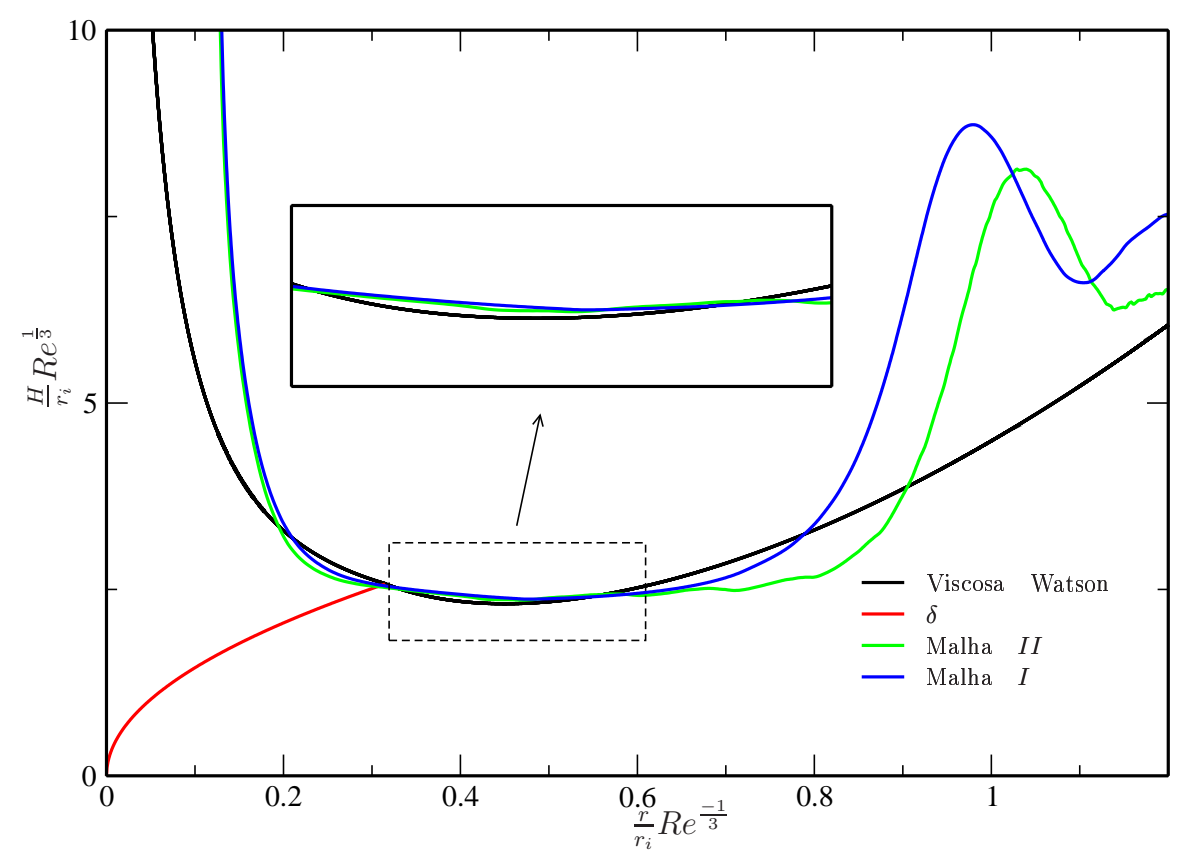

Figura 7.37: Comparação entre as soluções analítica de Watson (1964) [82] e numéricas obtidas pelo esquema FDHERPUS para o problema de ressalto hidráulico circular.

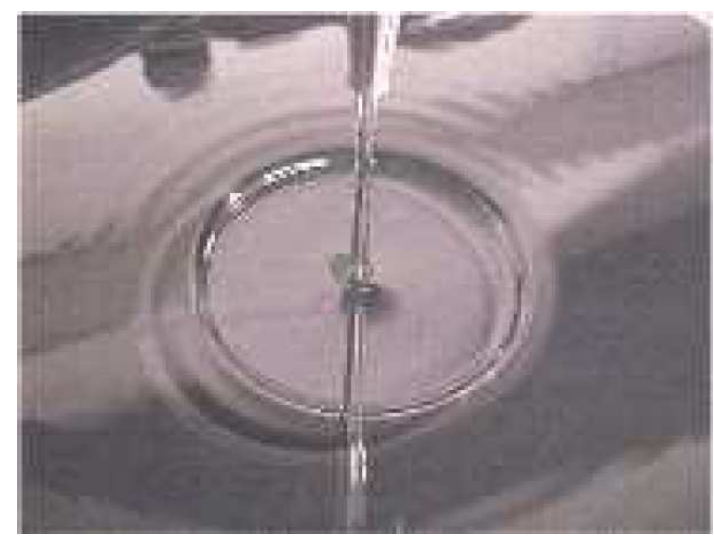

$(a)$

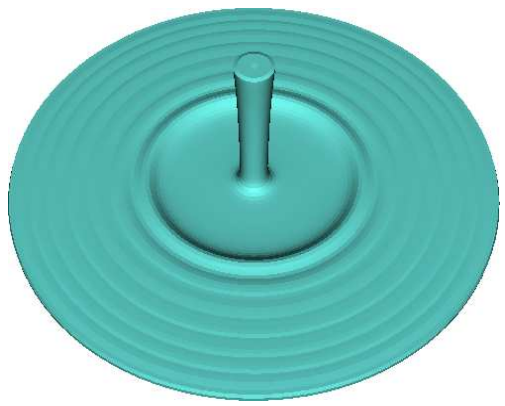

(b)

Figura 7.38: Ilustração dos resultados experimentais (fig. (a)) e numérico (fig. (b)) para o problema de ressalto hidráulico circular. 


\section{CAPÍTULO 8 \\ Resultados numéricos de problemas 3D complexos}

Neste capítulo são apresentados resultados numéricos para escoamentos incompressíveis tridimensionais complexos envolvendo superfícies livres móveis modelados pelas equações de Navier-Stokes (2.28). Esses resultaaods foram obtidos utilizado o ambiente computacional Freeflow de Castelo et al. (2000) [10] e utilizando a metodologia GENSMAC (ver Tomé e Mckee (2001) [71]), equipado com a nova estratégia upwind FDHERPUS. Mais uma vez, o esquema FDHERPUS foi escolhido por apresentar os melhores resultados em equações gerais de conservação hiperbólicas. A ênfase aqui é verificar se o esquema FDHERPUS aproxima bem as não linearidades nas equações completas (3D) de Navier-Stokes. Os problemas considerados foram: colapso de uma bloco de fluido, ressalto hidráulico e jato livre planar a baixo número de Reynolds. São comparados, os resultados obtidos, com dados teóricos, numéricos e experimentais consagrados na literatura.

\subsection{Colapso de um bloco de fluido}

Considera-se um bloco de fluido em equilíbrio hidrostático confinado entre paredes rígidas impermeáveis sob à ação de um campo gravitacional (problema este, similar ao resolvido na Seção 7.3.2). Uma representação esquemática do problema é apresentada na Figura 8.1. Para a simulação do problema os seguintes dados foram considerados:

- Malha: $150 \times 50 \times 80$ células computacionais ;

- Dimensão do domínio: $0.3 m \times 0.1 m \times 0.16 m$;

- Escala de comprimento: $L_{0}=0.1 \mathrm{~m}$;

- Escala de velocidade: $U_{0}=\sqrt{g L_{0}}=0.99045444 \mathrm{~m} / \mathrm{s}$;

- Número de Reynolds: $R e=99045.444$; 
- Condição de contorno free - slip aplicada nas paredes rígidas;

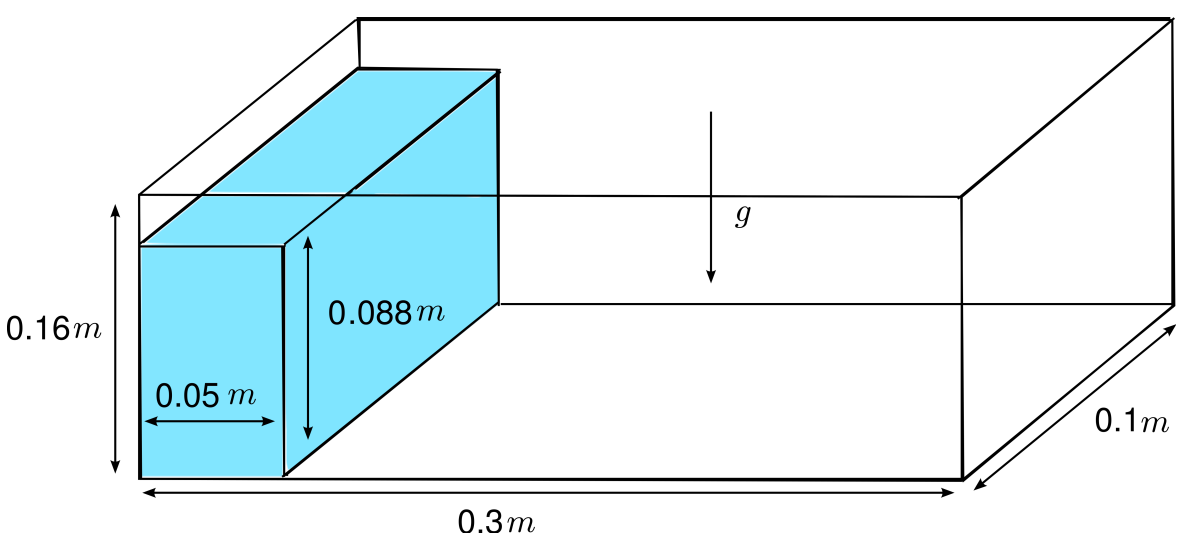

Figura 8.1: Representação esquemática do colapso de um bloco de fluido sob à ação da gravidade.

Nas Figuras 8.2-8.4 são mostrados os campos das velocidades nas direções $x, z$, o campo de pressão e a evolução da superfície livre do fluido. Conclui-se que o esquema FDHERPUS consegue simular sem instabilidades na superfície livre o colapso de um bloco de fluido; o que dá confiança para a simulação com o esquema FDHERPUS de outros problemas mais complexos em dinâmica de fluidos.
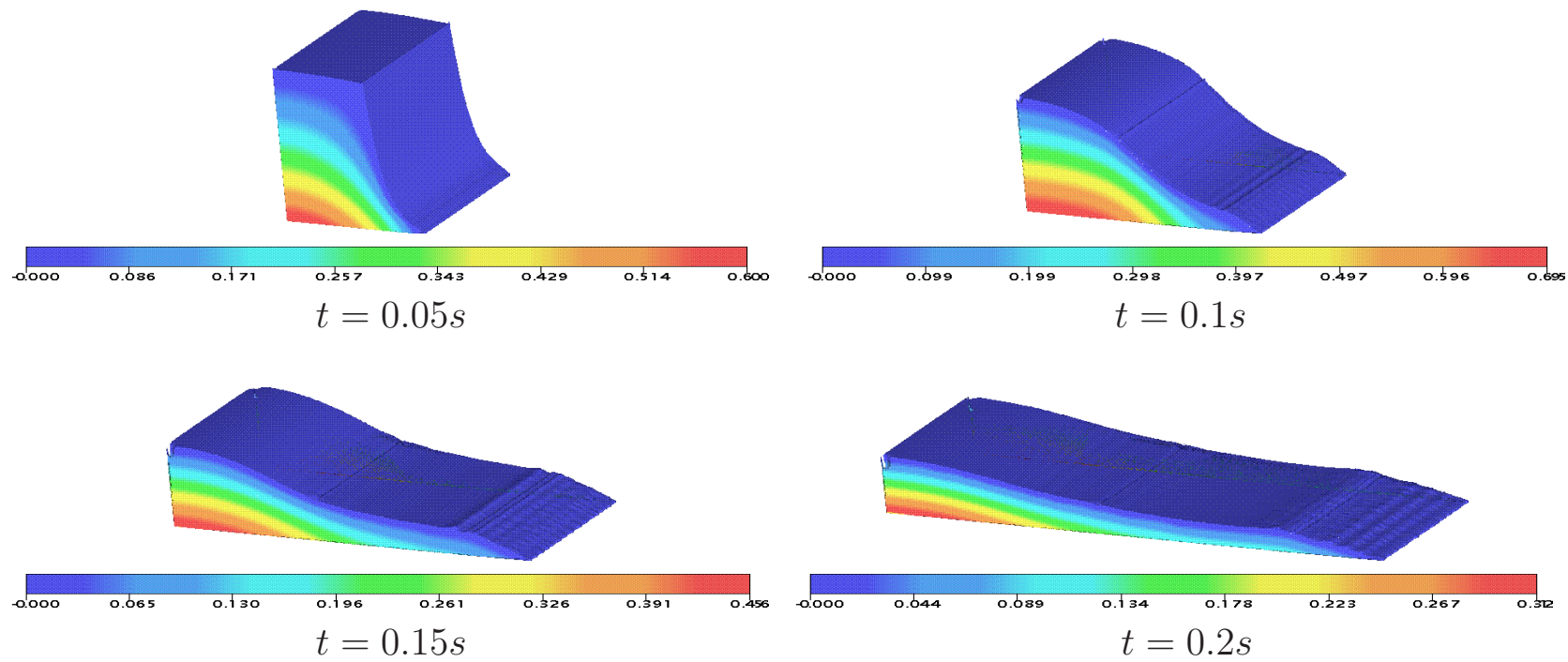

Figura 8.2: Campo da pressao para o problema de colapso de um bloco de fluido.

Para avaliar o desempenho do esquema FDHERPUS, os resultados numéricos obtidos foram confrontados com os resultados apresentados por Colagrossi (2003) [12] para o espalhamento 

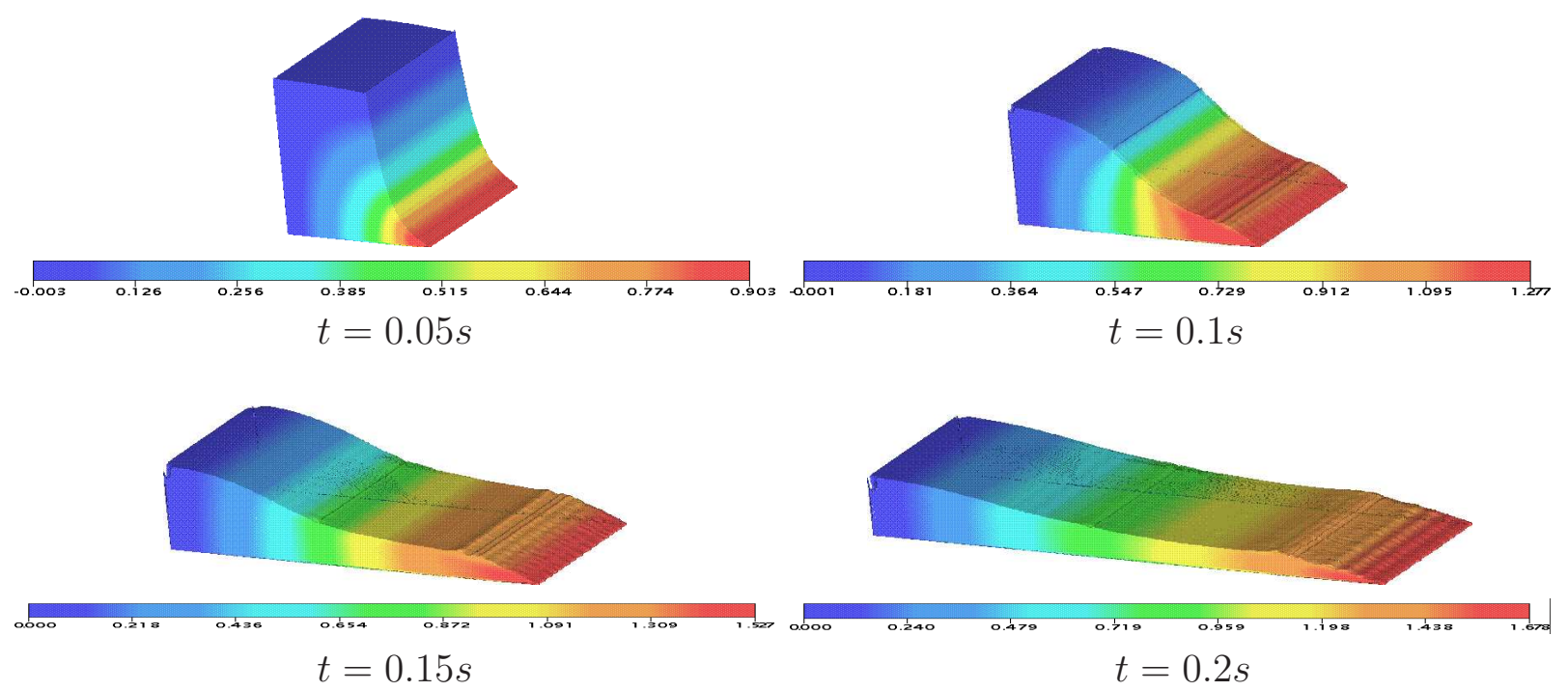

Figura 8.3: Campo da velocidade na direção $x$ para o problema de colapso de um bloco de fluido.

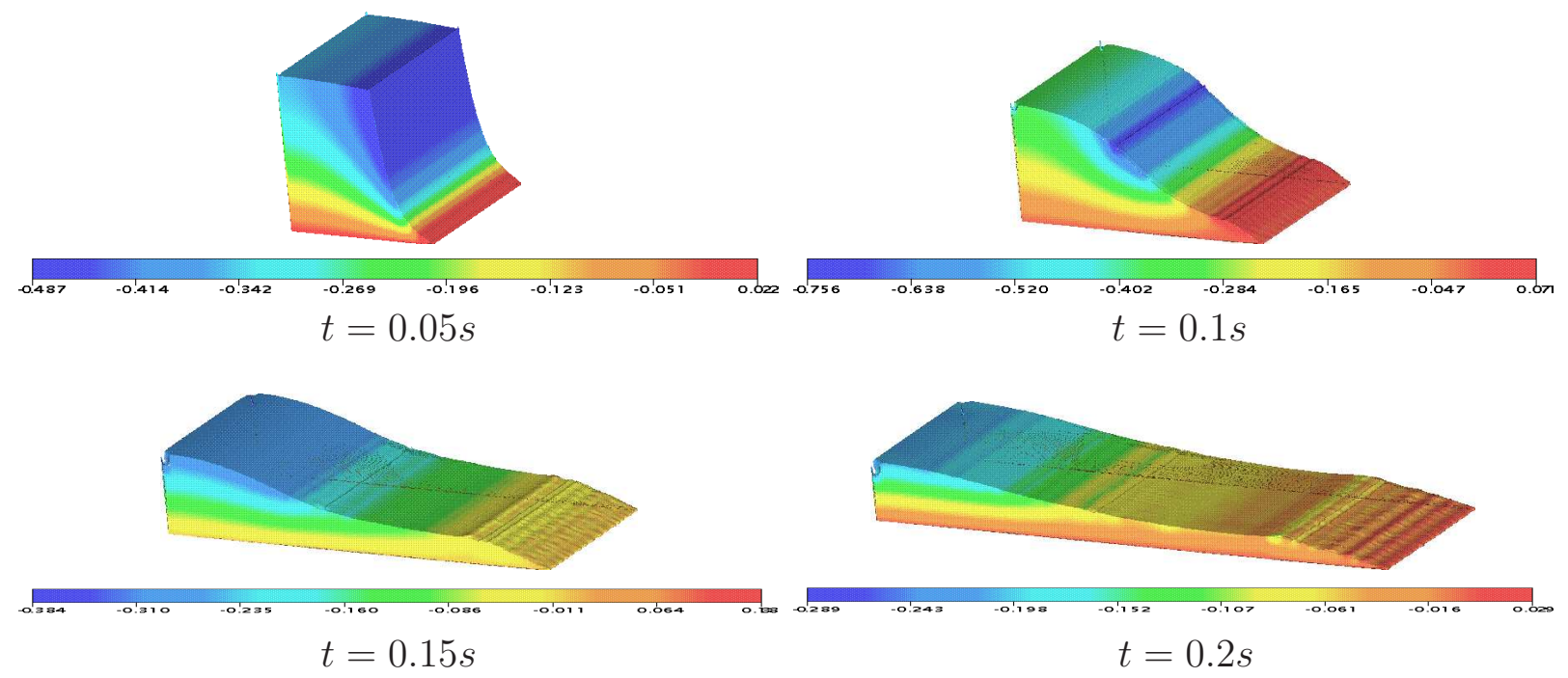

Figura 8.4: Campo da velocidade na direção $z$ para o problema de colapso de um bloco de fluido. 
horizontal $\left(x_{\max }\right)$, como é mostrado na Figura 8.5. Pode-se avaliar por esses dados que a solução obtida com o esquema FDHERPUS está em boa concordância com os resultados da literatura.

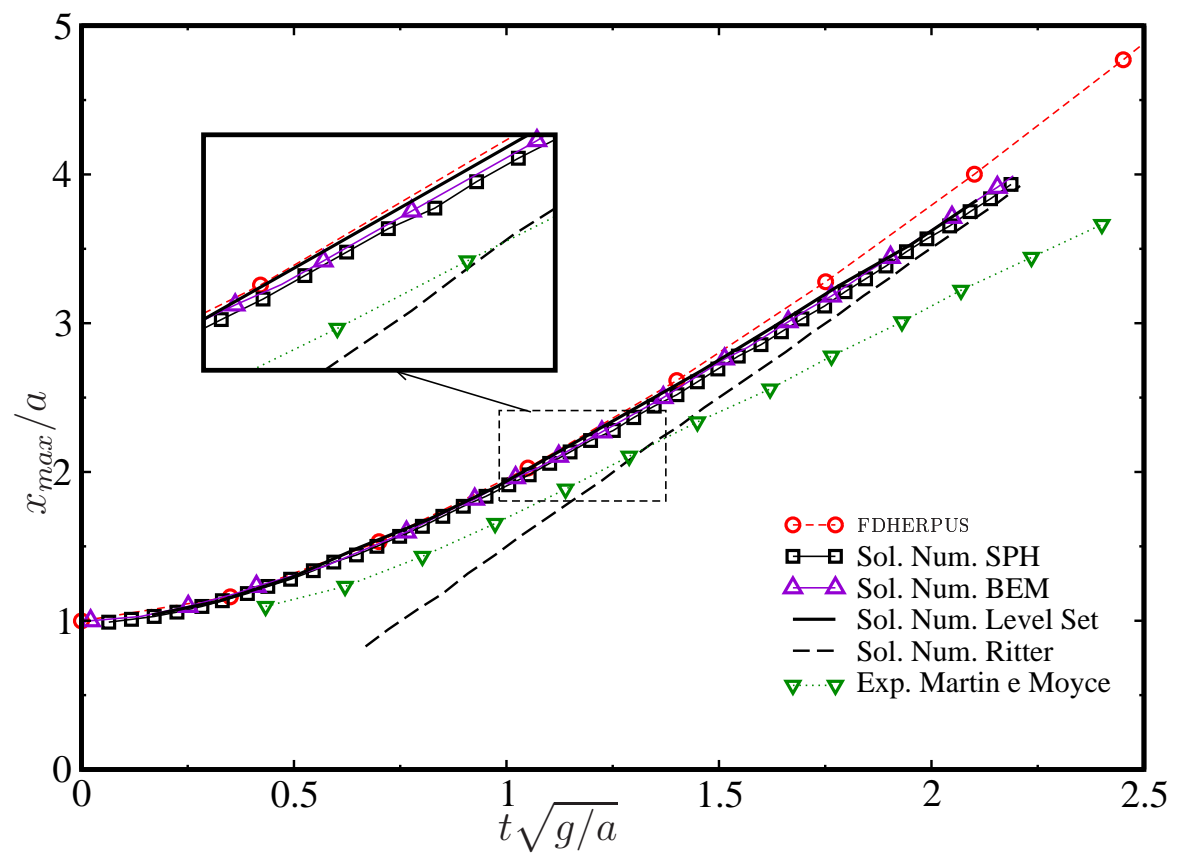

Figura 8.5: Comparação das soluções numéricas obtida com o FDHERPUS e os resultados consagrados da literatura.

\subsection{Ressalto hidráulico circular}

Uma simulação do ressalto hidráulico circular (já simulado na Seção 7.3 .4 para o caso de problemas em geometrias radiais) é apresentada nesta seção. Foi adotada a condição de contorno no - slip sobre a superfície rígida, e os seguintes dados foram adotados:

- Malha: $120 \times 120 \times 10$ células computacionais;

- Dimensão do domínio: $0.6 m \times 0.6 m \times 0.055 m$;

- Escala de comprimento: $L_{0}=2 r_{i}=0.05 m$;

- Raio do injetor: $r_{i}=0.025 m$

- Escala de velocidade: $U_{0}=1.0 \mathrm{~m} / \mathrm{s}$;

- Número de Reynolds: $R e=1000$;

Na Figura 8.6 está mostrado a comparação do resultado numérico via Freeflow (à esquerda) e o experimental (à direita) de Ellegard (1999) [18], para um tempo final de simulação $t=1.35 \mathrm{~s}$. 
Vê-se que o esquema FDHERPUS simulou com sucesso o salto hidráulico circular, incluindo as instabilidades físicas além da descontinuidade. Na Figura 8.7 é ilustrado o escoamento para os tempos $t=0.25, t=0.75$ e $t=1.25$.
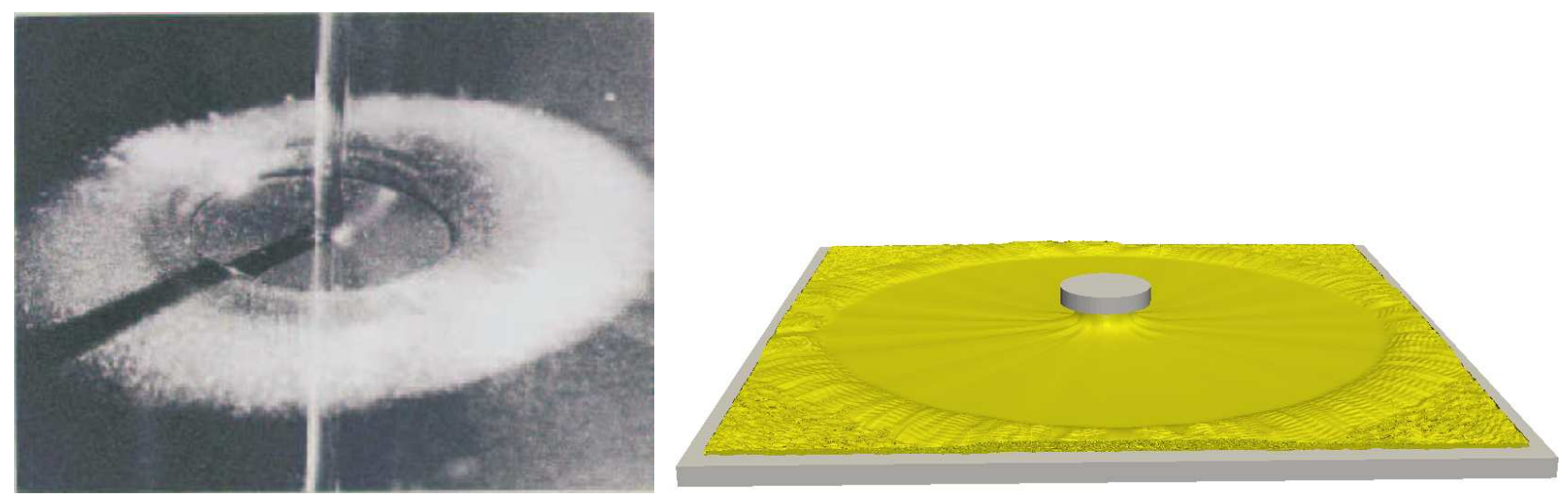

Figura 8.6: Comparação qualitativa entre os resultados experimental e numérico para o problema de ressalto hidráulico circular.

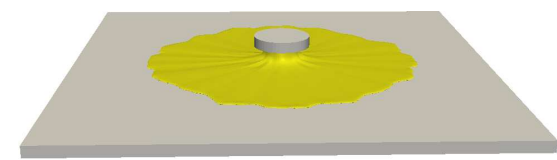

$t=0.25$

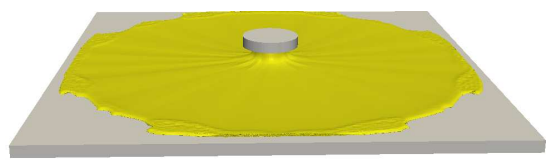

$t=0.75$

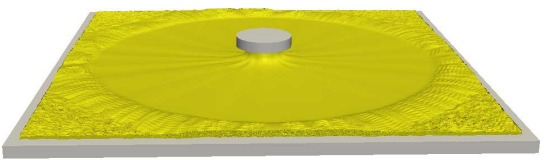

$t=1.25$

Figura 8.7: Evolução do escoamento mostrando a formação da descontinuidade.

\subsection{Jato planar oscilante}

Encerram-se os resultados tridimensionais mostrando a simulação computacional, usando o código Freeflowde adaptado com o esquema FDHERPUS, de um problema clássico interessante em mecânica dos fluidos: as oscilações de um jato planar a baixos números de Reynolds. Essas oscilações são instabilidades físicas e tem várias aplicações (ver, por exemplo, as referências $[55,80])$. Hoje em dia este problema fornece um excelente teste para validar métodos numéricos para escoamentos altamente viscosos envolvendo superfícies livres arbitrárias. Nos experimentos feitos por Cruickshank (1987) [15], para o caso do injetor circular, as instabilidades aparecem quando o fluido é suficientemente viscoso e o injetor situa-se à uma certa distância da superfície sólida. No caso planar, há muita controvérsia sobre as condições para as instabilidades. É importante observar que, muito embora o esquema FDHERPUS tenho sido proposto para problemas a altos valores do número de Reynolds, ele fornece bons resultados para escoamentos incompressíveis a baixos Reynolds $(R e<1)$. A evolução do escoamento a $R e=0.72$ simulado 
aqui, cujo modelo está ilustrado na Figura 8.8, é apresentada na Figura 8.9. Os seguintes dados foram adotados na simulação, para capturar as instabilidades físicas, a saber:

- Malha: $146 \times 66 \times 213$ células computacionais;

- Dimensão do domínio: $0.730 m \times 0.330 m \times 1.065 m$;

- Dimenções do injetor: $d=0.2 m, L_{i}=0.02 m$ e $h_{i}=0.05 m$

- Escala de comprimento: $L_{0}=0.02 m$;

- Escala de velocidade: $U_{0}=1.0 \mathrm{~m} / \mathrm{s}$;

- Número de Reynolds: $R e=0.72$;

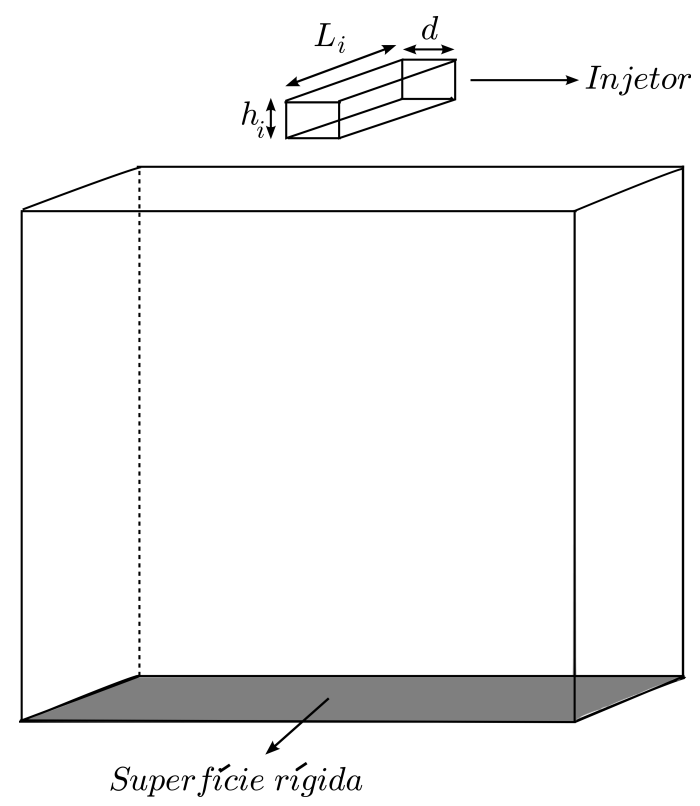

Figura 8.8: Representação esquemática para a simulação de um jato planar oscilante.

Como pode ser visto na Figura 8.9, as instabilidades físicas foram simuladas com sucesso. E os dados computacional e experimental apresentados nas Figuras 8.10 e 8.11, respectivamente, corroboram a previsão do jato planar oscilante obtida com o código Freeflow 3D suplementado com o esquema FDHERPUS. 

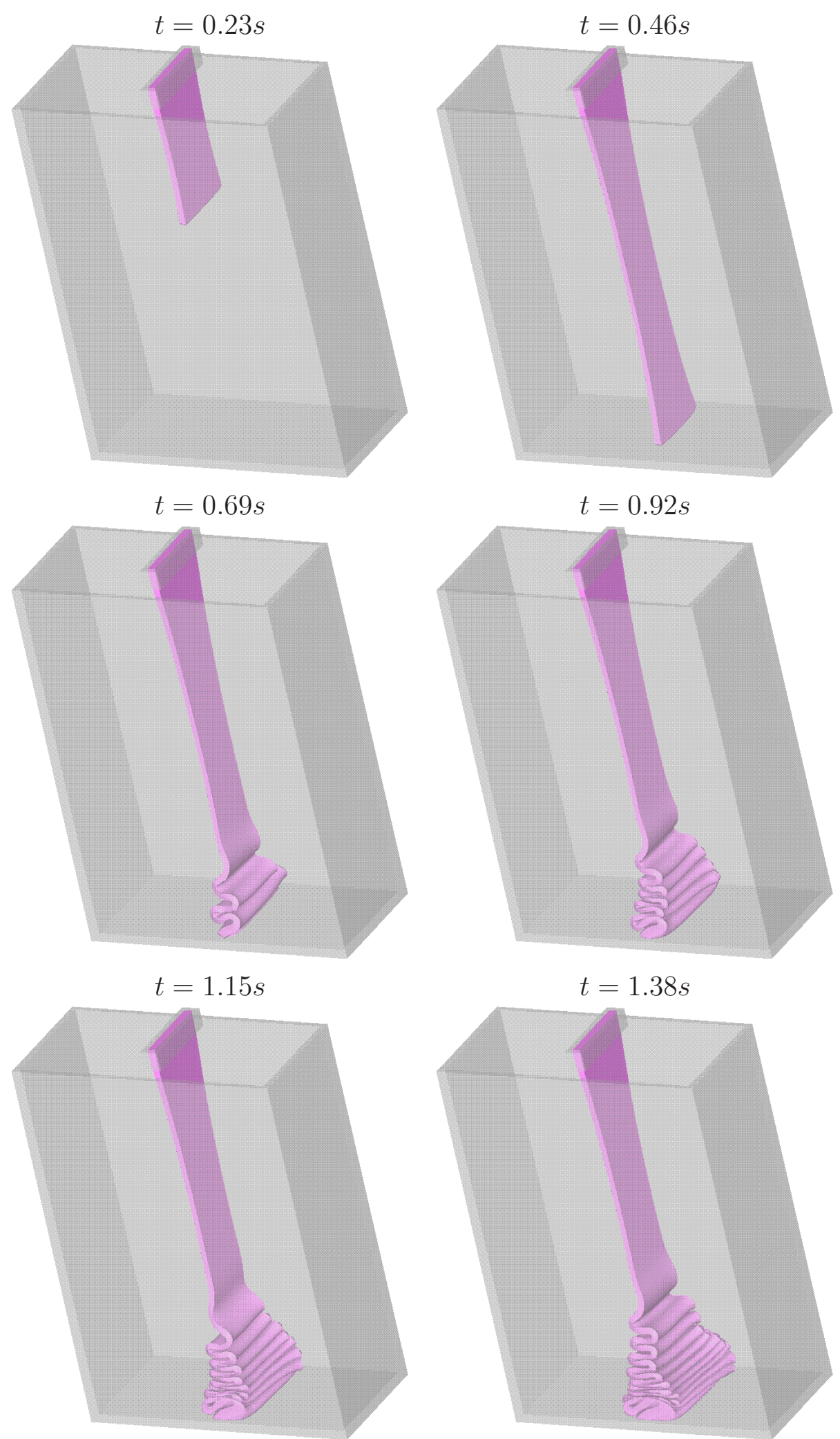

Figura 8.9: Resultados para o esquema FDHERPUS para o fenômeno do jato planar oscilante. 


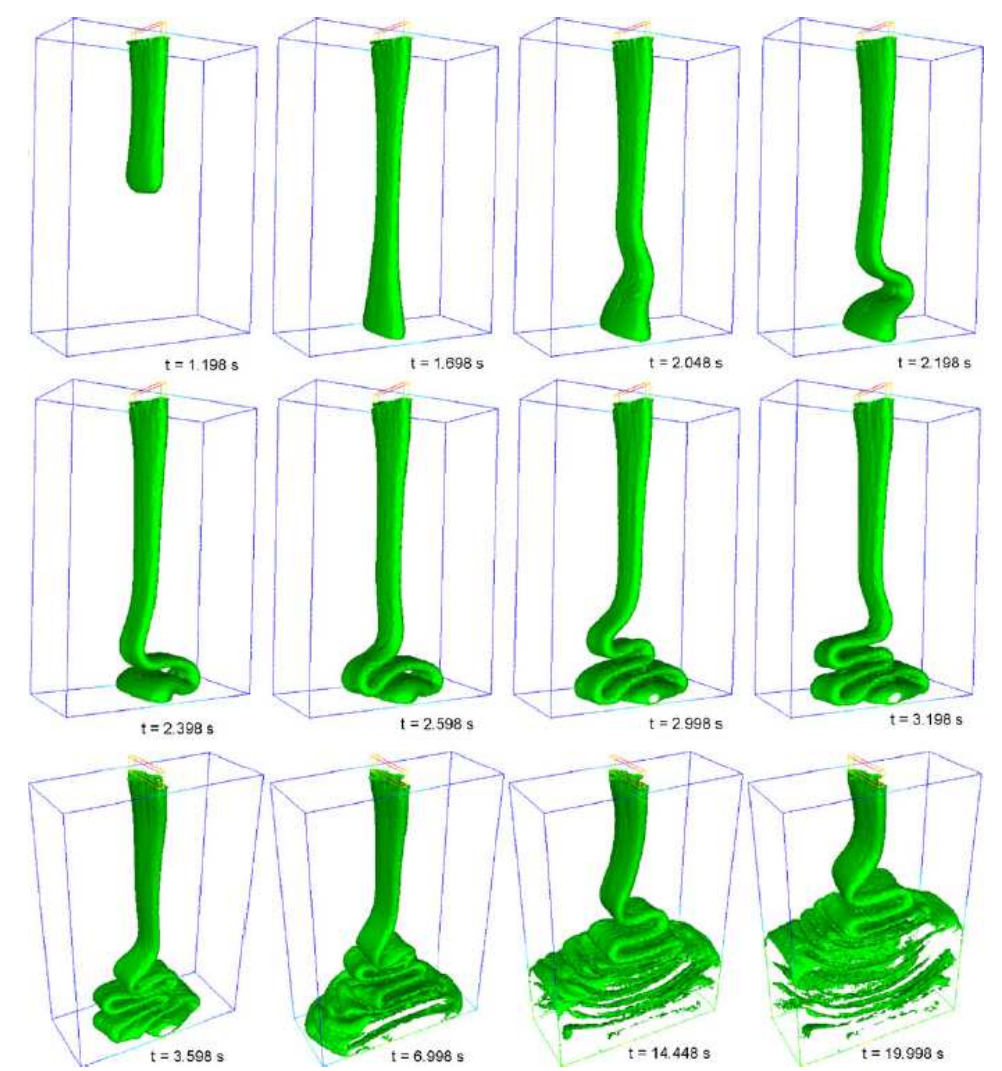

Figura 8.10: Simulação computacional de [80] para um jato planar oscilante.

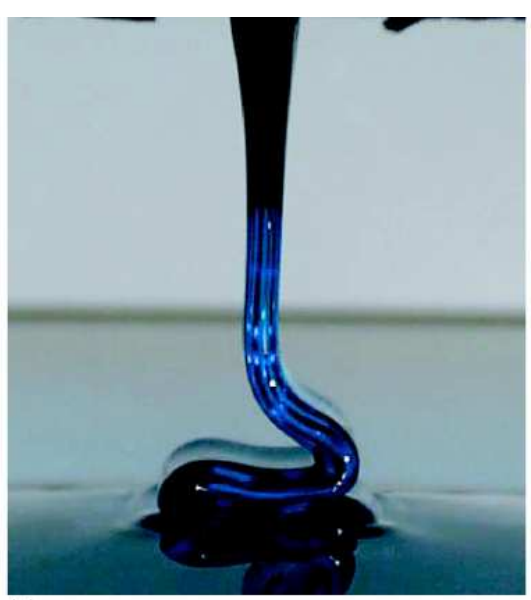

Figura 8.11: Resultado experimental de [54] para um jato planar oscilante. 


\section{Considerasões finais e trabalhos futuros}

O objetivo neste projeto de mestrado foi estudar esquemas upwind de captura de descontinuidades para aproximar termos convectivos nas equações que modelam problemas de dinâmica dos fluidos. E o objetivo maior foi fazer um estudo preliminar e profundo para, então, fornecer três esquemas particulares (nomeados como FDHERPUS, CSPUS e RUS) para a simulação numérica de problemas complexos em CFD. Os limitadores de fluxo correspondentes desses esquemas foram implementados no ambiente de simulação CLAWPACK para a resolução de leis de conservação hiperbólicas nos casos 1D e 2D. As versões desses esquemas em variáveis normalizadas de Leonard foram implementadas no ambiente de simulação Freeflow para a simulação computacional de escoamentos incompressíveis com superfícies livres móveis 2D, 2D-1/2 e 3D. As principais conclusões do trabalho são resumidas como segue:

1. Os esquemas (alternativos) FDHERPUS, CSPUS e RUS são capazes de resolver uma gama ampla de problemas em CFD, controlando oscilações numéricas próximas a descontinuidades e introduzindo pouca viscosidade numérica; suas formulações envolvem uma molécula computacional de três pontos fixos por fluxo numérico. Os esquemas podem ser usados como uma opção em algoritmos numéricos para economizar recursos computacionais. Foi introduzida uma modificação do esquema RUS (nomeada como MRUS) em termos dos gradientes consecutivos, e os resultados mostraram que esse esquema modificado forneceu melhores resultados que o original RUS.

2. Os três esquemas FDHERPUS, CSPUS e RUS são simples de implementar em códigos multidimensionais, tais como CLAWPACK e Freeflow, e fornecem soluções aceitáveis para sistemas hiperbólicos e parabólicos gerais; é apresentada também uma variedade de dados de simulações numéricas com o uso desses esquemas, o que permitirá no futuro pesquisadores comparar seus dados com os aqui apresentados; 
3. Dos problemas testes resolvidos, conclui-se que, em comparação com os esquema CSPUS e RUS, o esquema FDHERPUS forneceu os melhores resultados; resolvendo melhor pontos extremos (picos e vales). Quando aplicado na simulação de problemas de escoamentos incompressíveis com superfícies livres, o esquema FDHERPUS forneceu resultados encorajadores. Por exemplo, na resolução computacional do ressalto hidráulico circular o esquema FDHERPUS se comportou bem mostrando ser uma ferramenta útil para simular problemas complexos.

Em resumo, a realização do projeto propiciou uma atualização dos códigos CLAWPACK e Freeflow com novos esquemas de captura de descontinuidades, e que podem ser utilizados por todos os pesquisadores do grupo de pesquisa em dinâmica dos fluidos do LMACC do ICMC-USP e que se mantenham em constante desenvolvimento.

Para o futuro, pretende-se investigar os esquemas FDHERPUS, CSPUS, RUS e MRUS na resolução numérica de problemas mais complexos em fluidodinâmica, tais como aqueles que modelam problemas 3D de escoamentos turbulentos-viscoelásticos com superfícies livres móveis. Pretende-se também reunir os principais resultados obtidos neste trabalho de mestrado e submetê-los a uma revista internacional. 
CAPÍTUlO 10

\section{Produção científica associada}

Ao longo deste trabalho se concentrou esforços na produção de artigos científicos, os quais foram publicados/submetidos em anais de congressos. Estes trabalhos seguem listados abaixo, acompanhados de seus respectivos resumos.

\subsection{Artigos e resumos}

\subsubsection{Artigos publicados anais de congressos}

- NARváez, R. J. P., Bertoco J., Ferreira, G. V., Utilização do Polinômio de Hermite do Quinto Grau como Esquema Upwind: Aplicação em Escoamentos Com Superfícies Livres, CMAC 2011 - Congresso de Matemática Aplicada e Computacional, 2011, Uberlândia/MG.

Resumo: O trabalho apresenta um novo esquema upwind para a discretização dos termos convectivos não lineares das equações de Navier-Stokes 2D. Para o desenvolvimento do esquema, denominado HUS, utilizam-se o polinômio de Hermite do quinto grau e os critérios de estabilidades TVD/CBC. Como teste preliminar, o novo esquema é aplicado na solução do colapso de uma coluna de fluido sob à ação do campo gravitacional. Os resultados numéricos indicam que o esquema HUS é uma técnica promissora para problemas mais complexos em fluidodinâmica.

- Juliana Bertoco, Gabriela A. Reis, Rodolfo J. P. Narváez, Valdemir G. Ferreira, Simulação de escoamentos incompressiveis usando função racional para aproximar termos convectivos, CMAC 2011 - Congresso de Matemática Aplicada e Computacional, 2011, Uberlandia/MG.

Resumo: O trabalho trata da simulação de escoamentos incompressíveis usando uma função racional para aproximar os termos convectivos nas equações de Navier-Stokes. O esquema é baseado nos critérios de estabilidade TVD/CBC e doravante denominado RATIUS. Neste trabalho, o esquema foi testado na simulação de um jato livre sobre uma superfície rígida im- 
permeável. Os resultados obtidos mostram que o esquema RATIUS funciona bem em problemas incompressiveis com superfícies livres móveis.

- Candezano M. A. C., Corrêa, L., NARVÁEZ R. J. P., Ferreira G. V., Application of new polynomial upwind schemes to Rayleigh-Taylor instability, CMAC 2010 - Congresso de Matemática Aplicada e Computacional, 2011, Uberlândia/MG.

Abstract: The Rayleigh-Taylor instability is one of the most typical $2 D$ benchmark test for numerical schemes due for this complex structures. We present in this work a numerical solution of this representative problem using four different type of TVD upwind schemes. We determine too that two recently TVD upwind polynomial schemes are able to compute this complex structures without spurious oscillations and with a good resolution as it is made using another high-resolution techniques, e.g. ENO/WENO.

- Candezano M. A. C., NARVÁEZ R. J. P., Juliana Bertoco, Ferreira G. V., Três Novos Limitadores de Fluxo para Sistemas de Leis de Conservação, ENAMA 2011 - Encontro Nacional de Análise Matemática e Aplicações, 2011, São Carlos/SP.

Abstract: Muitos problemas em física e em engenharia são modelados por leis de conservação hiperbólicas, cujos termos não lineares (convectivos) influenciam sobremaneira a solução. A busca por um limitador de fluxo simples que aproxime bem esses termos e introduza pouca dissipação numérica, sem a presença de oscilações não-físicas, tem sido um desafio permanente para a comunidade científica moderna em dinâmica dos fluidos computacional. Vários limitadores de fluxo têm sido desenvolvidos nas últimas décadas, e dentre eles destaca-se o de van Albada [75]. Nesse trabalho, três novos limitadores de fluxo são apresentados, a saber: o HERMITE - baseado no polinômio interpolador de Hermite; o SPLINE - baseado na interpolação spline cúbica; e RUS (limitador teste) - obtido de uma função racional. Nesse trabalho, o sistema de equações de Euler $2 D$ é resolvida numericamente para o problema das instabilidades de Rayleigh Taylor, usando o algoritmo de propagação de ondas (uma variação do método de Godunov com termo de correção) implementado no ambiente de simulação CLAWPACK de LeVeque [41].

- Miguel A. C. Candezano, Patrícia Sartori, Laís Corrêa, Giseli A. B. Lima, Rodolfo Pérez e Valdemir G. Ferreira, A numerical study of some recently introduced TVD upwinding schemes with applications in fluid dynamics problems, COBEM 2011 - 21st International Congress of Mechanical Engineering, 2011, Natal/RN.

Abstract: An important issue in computational fluid dynamics is the appropriate approximation of the convection phenomena. For this, the TVD schemes are alternatives to the ENO/WENO techniques due to the robustness, low cost and simplicity of implementation. Within this scenario, the aim of the our work is to present a numerical study of some recently introduced polynomial TVD upwinding schemes - namely TOPUS and SDPUS - with appli- 
cations in fluid dynamics problems. By using these new upwind schemes, numerical results for nonconvex nonlinear problem, $2 D$ advection of scalars and $2 D$ Euler equations are presented. Comparison with the well recognized CFL-dependent ARORA-ROE and ADBQUICKEST schemes and the conventional SUPERBEE and MC schemes are assessed. The TOPUS and SDPUS upwind schemes are developed in the context of normalized variables (NV) of Leonard and satisfy TVD constraints of Harten.

- Rodolfo P. Narváez, Valdemir G. Ferreira, Uma nova técnica de interpolação upwind para termos convectivos, ERMAC 2012 - Encontro Regional de Matemática Aplicada e Computacional, 2012, Botucatu/SP.

Resumo: O trabalho apresenta um novo esquema upwind de alta resolução denominado RUS, para resolver numericamente equações de conservação. Para o desenvolvimento do esquema, utilizam-se uma função racional e os critérios de estabilidades TVD/CBC. Como teste preliminar, apresenta-se os resultados numéricos obtidos pelo esquema RUS para resolver o sistema não-linear de Euler 2D, a saber, o problema Four-shocks problem e na solução das equações de Navier-Stokes para a simulação de escoamentos incompressíveis 3D. Os resultados numéricos indicam que o esquema RUS é uma técnica promissora para problemas mais complexos em fluidodinâmica.

- Juliana Bertoco, Gabriela A. dos Reis, Rodolfo J. P. Narváez, Valdemir G. Ferreira, Aplicação de um esquema upwind na resolução numérica de equações de conservação com o uso de adaptação temporal, ERMAC 2012 - Encontro Regional de Matemática Aplicada e Computacional, 2012, Botucatu/SP.

Resumo: Neste trabalho, apresenta-se um esquema upwind do tipo função racional e seu respectivo limitador de fluxo para aproximar termos convectivos em equações de conservação. Mostra-se ainda uma estratégia computacional para melhorar o passo temporal em escoamentos a baixos valores do número de Reynolds. Três testes numéricos são resolvidos; as instabilidades de Rayleight-Taylor e os problemas fountain flow e ressalto hidráulico circular. Em todos os testes pode-se aferir que o esquema upwind apresentado é robusto, tanto para problemas a altos valores de Reynolds, quanto para baixos valores, capturando bem os fenômenos físicos.

-Ferreira G. V., NARVÁEZ R. J. P., Candezano M. A. C., Numerical Simulation of Complex Problems in Fluid Dynamics, CONEM 2012 - VII Congresso Nacional de Engenharia Mecânica, 2012, São Luís/MA.

Abstract: This article is concerned with the numerical simulation of com plex problems in fluid dynamics using a recently introduced convection upwind scheme. The scheme is developed by using Hermite interpolation and $C B C / T V D$ stability criteria; it is implemented in the context of finite volume and finite difference and into two simulation environment, namely: CLAWPACK, for 1D/2D hyperbolic conservation laws; and Freeflow, for 3D incompressible flows. The performance of the scheme is investigated by solving transport of scalars, non-linear Burguers 
and Euler equations. Comparisons with the results provided by the well established van Albada scheme are also presented. Then, as application, the scheme is employed to simulate 3D moving free surface Newtonian flows, such as broken dam and circular hydraulic jump. The numerical results demonstrate that the upwind scheme discussed in this article works well in several PDEs of fluid dynamics.

- Rodolfo P. Narváez, Juliana Bertoco, Valdemir G. Ferreira, Numerical simulation of complex problems in fluid dinamics, CNMAC 2012 - XXXIV Congresso Nacional de Matématica Aplicada e Computacional, 2012, Águas de Lindóia/SP.

Resumo: O trabalho apresenta um novo limitador de fluxo baseado no polinômio interpolador de Hermite do quinto grau. O objetivo é disponibilizar esse novo limitador à comunidade científica em dinâmica dos fluidos computacional para simulação de problemas complexos na formulação compressivel, incompressivel ou ambas. Para o desenvolvimento do limitador, utilizam-se os critérios de estabilidade TVD de Harten [30] e de limitação CBC de Gaskell e Lau [23]. Resultados numéricos são apresentados para o problema de um jato em astrofísica a um alto valor do número de Mach, o qual é modelado pelas equações hiperbólicas de Euler 2D. Esses resultados indicam que o novo esquema RUS pode ser bastante útil em outros problemas mais complexos.

\subsubsection{Artigos submetidos em periódicos}

- Miguel A. Caro Candenazo, Rodolfo J. P. Narváez and Valdemir G. Ferreira, Numerical simulation of complicated PDEs in fluid dynamics, Boletín de la Asociación Matemática Venezolana, 2012, Caracas/Venezuela.

Abstract: This article is concerned with the numerical simulation of complex problems in fluid dynamics using a recently introduced convection upwind scheme. The scheme is developed based on a rational interpolation function and $C B C / T V D$ stability criteria; it is implemented in the context of finite volume/finite difference and into two simulation environment namely: CLAWPACK, for $1 D / 2 D$ systems of hyperbolic conservation laws; and Freeflow, for $3 D$ incompressible Newtonian flows. The performance of the scheme is investigated in solving transport of scalars, non-linear Burguers and Euler equations. Comparisons with the results provided by the well established van Albada scheme are also presented. As application, the scheme is then employed to simulate 3D moving free surface Newtonian flows (broken dam and circular hydraulic jump). The results demonstrate that the upwind scheme discussed in this article works well in several PDEs of fluid dynamics. 


\section{CLAWPACK}

O cálculo de flidos inviscidos, os quais sao modelados pela a equação

$$
\frac{\partial \boldsymbol{U}}{\partial t}+\frac{\partial \boldsymbol{F}(\boldsymbol{U})}{\partial x}=0
$$

são resolvidos utilizando o software CLAWPACK (Conservation LAWs PACKage) de Leveque (2004) [41]; suplementado com os esquemas upwind em forma de limitadores de fluxo. Esse código, foi desenvolvido na Universidade de Washington para resolver sistemas de equações hiperbólicas de EDPs. O software, no contexto da metodologia de volumen finitos, utiliza o metodo de Godunov's com um termo de correção. A seguir, uma descrição da implementação das funcões limitadores de fluxo no pacote CLAWPACK. Por simplicidade e sem perda de generalidade, a versão unidimensional para o sistema hiperbólico A.1, que na forma quase-linear é dada como

$$
u_{t}+A(x, t) u_{x}=0
$$

em que $A(x, t)$ é a matrix Jacobina $A(x, t)=F^{\prime}(u(x, t))$. A reconstrução de alta ordem para a variavel $u$, implementado no pacote CLAWPACK, vem dado como

$$
\underbrace{u_{i}^{n+1}=u_{i}^{n}-\frac{\delta_{t}}{\delta_{x}}\left[A^{-} \Delta u_{i+\frac{1}{2}}+A^{+} \Delta u_{i+\frac{1}{2}}\right]}_{\text {Metodo de Godunov de } 1^{\text {er }} \text { ordem }}-\frac{\delta_{t}}{\delta_{x}}\left(\tilde{F}_{i+\frac{1}{2}}-\tilde{F}_{i-\frac{1}{2}}\right)
$$

onde $A^{-} \Delta u_{i-\frac{1}{2}}$ e $A^{+} \Delta u_{i+\frac{1}{2}}$ são as flutuações obtida da solução do problema de Riemann para $i= \pm \frac{1}{2}$ são dados como 


$$
\begin{aligned}
& A^{-} \Delta u_{i+\frac{1}{2}}=\sum_{q=1}^{m}\left(\lambda^{q}\right)^{-} W_{i-\frac{1}{2}}^{q} \\
& A^{+} \Delta u_{i-\frac{1}{2}}=\sum_{p=1}^{m}\left(\lambda^{q}\right)^{+} W_{i-\frac{1}{2}}^{q}
\end{aligned}
$$

onde $m$ é o numero de ondas $W^{q}$ viajando a velocidade $\lambda^{q},\left(\lambda^{q}\right)^{-}=\min (\lambda, 0)$ e $\left(\lambda^{q}\right)^{+}=\max (\lambda, 0)$, na que $\lambda$ é o autovalor da matriz A. No software CLAWPACK, resolvedores de Riemann, primeiro calcula ondas e velocidades e depois, calcula as flutuações retornadas por os resolvedores de Riemman, e com os valores conhecidos para os fluxos numéricos $\tilde{F}_{i-\frac{1}{2}}$ e $\tilde{F}_{i+\frac{1}{2}}$, o método na equação (A.3) é usado para calcular a reconstrução para a expressão $u_{i}^{n+1}$. Por exemplo, $\tilde{F}_{i-\frac{1}{2}}$ é calculado como

$$
\tilde{F}_{i-\frac{1}{2}}=\frac{1}{2} \sum_{p=1}^{m}\left|\lambda_{i-\frac{1}{2}}^{q}\right|\left(1-\frac{\delta_{t}}{\delta_{x}}\left|\lambda_{i-\frac{1}{2}}^{q}\right|\right) \tilde{W}_{i-\frac{1}{2}}^{q},
$$

onde $\tilde{W}_{i-\frac{1}{2}}^{q}$ (uma versão limitada da onda $W_{i-\frac{1}{2}}^{q}$ ) vem dada por

$$
\tilde{W}_{i-\frac{1}{2}}^{q}=\tilde{\alpha}_{i-\frac{1}{2}} \theta^{q}
$$

sendo $\theta^{q}$ o $q$-éssimo eigenvetor de $A$ e $\tilde{\alpha}_{i-\frac{1}{2}}$ um coficiente definido como

$$
\tilde{\alpha}_{i-\frac{1}{2}}=\alpha_{i-\frac{1}{2}} \psi\left(r_{i-\frac{1}{2}}^{q}\right) .
$$

Na equação (A.8), o cofiiciente $\alpha$ é claculado como $\alpha_{i-\frac{1}{2}}=\Theta^{-1}\left[u_{i}-u_{i-1}\right]^{T}$, sendo $\Theta$ a matriz de eigenvetores de $A$, e $\psi=\psi\left(r_{i-\frac{1}{2}}^{q}\right)$ é a função limitador de fluxo, a qual depende do sensor de suavidade

$$
r_{i-\frac{1}{2}}^{q}=\frac{r_{\alpha_{I-\frac{1}{2}}}}{\alpha_{i-\frac{1}{2}}}, I=\left\{\begin{array}{cc}
i-1, & \lambda_{i-\frac{1}{2}}^{q}<0 \\
i+1, & \lambda_{i+\frac{1}{2}}^{q}>0 .
\end{array}\right.
$$

Com um procedimento similar, pode-se calcular $\tilde{F}_{i+\frac{1}{2}}$. O limitador de fluxo $\psi$ que aparece na equação (A.8) corresponde neste trabalho a os limitadores de fluxo propostos e são implementados no software CLAWPACK utilizando para aquilo a equação (A.3). Esta equação pode ser extendida de maneira simples para casos multidimensionais (ver detalhes em LeVeque (1997) [40]). 


\section{Referências Bibliográficas}

[1] M. A. Alves, P. J. Oliveira, and F. T. Pinho. A convergent and universally bounded interpolation scheme for the treatment of advection. International Journal for Numerical Methods in Fluids, 41:47-75, 2003.

[2] A. Amasden and F. H. Harlow. The SMAC Method: A simplified MAC technique for incompressible fluid flow calculations. Journal of Computational Physics, 6(2):322-325, 1970.

[3] D. S Balsara and C. W. Shu. Monotonicity preserving weighted essentially non-oscilatory scheme with increasingly high order of accuracy. Journal of Computational Physics, 160:405-452, 2000.

[4] C. Berthon. Stability of the muscl schemes for the euler equations. Communications in Mathematical Sciences, 3:133-158, 2005.

[5] J. P. Boris and D. L. Book. Flux-corrected transport 1: SHASTA, a fluid-transport algorithm that works. Journal of Computational Physics, 11:38-69, 1973.

[6] J. P. Boris and D. L. Book. Flux-corrected transport III: Minimal error FCT methods. Journal of Computational Physics, 20:397-431, 1976.

[7] A. Bressan, G. G. Chen, and M. Lewicka. Nolinear Conservation Laws and Applications. Springer, 2011.

[8] R. L. Burden and J. D. Faires. Numerical Analysis. Richard Stratton, 2010.

[9] M. Cada and M. Torrilhon. Compact third-order limiter functions for finite volume methods. Journal of Computational Physics, 228:4118-4145, 2009.

[10] A. F. Castelo, M. F. Tomé, C. N. L. César, S. McKee, and J. A. Cuminato. Freeflow: An integrated simulation system for three-dimensional free-surface flows. Journal of Computers and Visualization in Science, 2:199-210, 2000. 
[11] A. J. Chorin. Numerical solution of the Navier-Stokes equations. Mathematics of Computation, 22:745-762, 1968.

[12] A. Colagrossi and M. Landrini. Numerical simulation of interfacial flow by smoothed paticle hydrodynamics. Journal of Computational Physics, 191:448-475, 2003.

[13] G. Colicchio, M. Landrini, and L. C. Chaplin. Level-set modelling of the air-water flow generated buy a surface piercing body. In Proceedings 8th International Conference on Numerical Ship Hydrodynamics, 2003.

[14] R. Courant, E. Isaacson, and M. Rees. On the solution of nonlinear hyperbolic differential equations by finite differences. Comunication on Pure and Applied Mathematics, 5:243-255, 1952.

[15] J. O. Cruickshank. Low-reynolds-number instabilities in stagnating jet flows. Journal of Fluid Mechanics, 193:111-127, 1987.

[16] M. Darwish and F. Moukalled. The x-schemes: a new consistent high-resolution formulation based on normalized variable moethodology. Computer Methods in Applied Mechanics and Engineering, 192:1711-1730, 2003.

[17] A. V. F. de Azevedo and C. S. Eschenazi. Leis de conservação com aplicações ao tráfego nas cidades. Técnicas computacinais para dinâmica dos fluidos: conceitos básicos e aplicações. II Bienal da Sociedade Brasileira de Matemática, Salvador 2004.

[18] C. Ellegaard, A. E. Hansen, A. Haaning, K. Hansen, A. Marcussen, and T. Bohr. Poligonal hydraulic jump. Nonlinearity, 12:1-7, 1999.

[19] V. G. Ferreira. Análise e Implementação de Esquemas de Convecção e Modelos de Turbulência para Simulação de Escoamentos Incompressiveis Envolvendo Superfícies Livres. PhD thesis, Universidade de São Paulo, 2001.

[20] V. G. Ferreira, F. A. Kurokawa, R. A. B. Queiroz, M.K. Kaibara, C.M. Oishi, J. A. Cuminato, A. Castelo, M. F. Tomé, and S. Mckee. Assessment of a high-order finite difference upwind scheme for the simulation of convection-diffusion problems. International Journal for Numerical Methods in Fluids, 60:1-26, 2009.

[21] V. G. Ferreira, R. A. B. Queiroz, F. A. Kurokawa, R. G. Cuenca, C. M. Oishi, G. A. B. Lima, and S. Mckee. An upwind differencing scheme for conservation laws and related fluid dynamics problems. Strathclyde Mathematics Research Report, No. 4, 2009.

[22] V. G. Ferreira, R. A. B. Queiroz, G. A. B. Lima, R. G. Cuenca, C. M. Oishi, and J. L. F. Azevedo. A bounded upwinding scheme for computing convection-dominated transport problems. Computers and Fluids, 57:208-224, 2012. 
[23] P. H. Gaskell and A. K. C. Lau. Curvature-compensated convective transport: SMART, a new boundedness preserving transport algorithm. International Journal for Numerical Methods in Fluids, 8:617-641, 1988.

[24] W. F. Godoy and P. E. Desjardin. On the use of flux limiters in the discrete ordinates methods for $3 \mathrm{~d}$ radiation calculations in absorbing and scattering media. Computational Physics, 229:3189-3213, 2010.

[25] M. Greco, M. Landrini, and O. M. Faltinsen. Impact flows and loads on ship-deck structures. Journal of Fluids and Structures, 2003.

[26] Y. Ha. Numerical methods for supersonic astrophysical jets. PhD thesis, Arizona State University, 2003.

[27] Y. Ha. Positive Scheme Numerical Simulation of High Mach Number Astrophysical Jets. Journal of Scientific Computing, 34:247-259, 2008.

[28] Y. Ha, C. L. Gardner, A. Gelb, and C. W. Shu. Numerical simulation of high mach number. astrophysical jets with radioactive cooling. Journal of Scientific Computing, 24 No.1:29-44, 2005.

[29] F. H. Harlow and E. Welch. Numerical calculation of time-dependent viscous incompressible flow of fluid with free surface. Physics of Fluids, 8:2182-2189, 1965.

[30] A. Harten. High resolution schemes for hyperbolic conservation laws. Journal of Computational Physics, 49:357-393, 1983.

[31] A. Harten. ENO scheme with subcell resolution. Journal of Computational Physics, 83:148-184, 1989.

[32] M. R. Hestenes and E. Stiefel. Methods of conjugate gradients for solving linear systems. Journal of Research of the National Bureau of Standards, 49:409-436, 1952.

[33] M. E. Hubbard and P. Garcia-Navarro. Flux difference splitting and the balancing of source terms and flux gradients. Journal of Computational Physics, 165:89-125, 2000.

[34] G. S. Jiang and C. W. Shu. Efficient implementation of weighted ENO schemes. Journal of Computational Physics, 126:202-212, 1996.

[35] M. Kurihara. On hydraulic jumps. Proceedings of Reports of the Research Institute for Fluid Engineering, Kyusyu Imperial Univaersity, 3:11-33, 1946.

[36] B. P. Leonard. The QUICK algorithm: a uniformly third-order finite difference method for highly convective flows. Computer Methods in Applied Mechanics and Engineering, 19:59, 1979. 
[37] B. P. Leonard. Simple high-accuracy resolution program for convective modelling of discontinuities. International Journal for Numerical Methods in Fluids, 8:1291-1318, 1988.

[38] R. J. LeVeque. CLAWPACK and AMRCLAW Software for High Resolution Godunov Methods. 4-th ntl. Conference on Wave Propagation, 1988.

[39] R. J. LeVeque. Numerical Methods for Conservation Laws. 1992.

[40] R. J. LeVeque. Wave propagation algorithms for multidimensional hyperbolic systems. Journal of Computational Physics, 131:327-353, 1997.

[41] R. J. LeVeque. Finite volumes methods for hyperbolic problems. Press Syndicate of the University of Cambridge, 2004.

[42] R. J. LeVeque, J. Langhset, M. Berger, D. McQueen, D. Calhoun, P. Blossey, and S. Mitran. Clawpack 5.0: software package designed to compute numerical solutions to hyperbolic partial differential equations using a wave propagation approach. http://www.amath.washington.edu/claw/, 2006.

[43] G. A. B. Lima, V. G. Ferreira, E. R. Cirilo, A. Castelo, M. A. C. Candenazo, I. V. M. Tasso, D. M. C. Sano, and L. V. A. Scalvi. A continuously differentiable upwinding schemes for the simulation of fluid flow problems. Applied Mathematics and Computation, 218:8614-8633, 2012.

[44] H. Lin and C. C. Chieng. Characteristic-based flux limiters of an essentially third-order flux-splitting method for hyperbolic conservation laws. International Journal for Numerical Methods in Fluids, 13:287-307, 1991.

[45] B. Liu, G. R. Lam, K. Y. Song, and R. S. Amano. On a higer-order bounded discretization scheme. International Journal for Numerical Methods in Fluids, 32:881-887, 2000.

[46] J. C. Martin and W. J. Moyce. An experimental study of the collapse of liquid columns on a rigid horizontal plane. Philosophical Transactions of the Royal Society A: Mathematical, Phisical an Engineering Sciences, 244:312-324, 1952.

[47] A. Murrone and H. Guillard. Behaivour of upwind scheme in their low Mach number limit: III. Preconditioneddissipation for a five equation two phase model. Computers and Fluids, 37:1209-1224, 2008.

[48] R. Peyret and T. D. Taylor. Computational methods for fluid flow. New York, 1983.

[49] S. Piperno and S. Depeyre. Criteria for the Desing of Limiters Yielding Efficient High Resolution TVD Schemes. Computer and Fluids, 27:183-197, 1998. 
[50] J. Qiu and C. W. Shu. Finite defference WENO schemes with lax-wendroff-type time discretizations. Society for Industrial and Applied Mathematics, 24:2185-2198, 2003.

[51] A. Quarteroni and F. Saleri. Cálculo Cientifico com Matlab e Octave. Springer, 2007.

[52] R. A. B. Queiroz and V. G. Ferreira. Development and testing of high-resolution upwind schemes: Upwind schemes for incompressible free surface flows. 284 p. 1 ed. VDM Verlag Dr Muller, 2010.

[53] A. Rai, B. S. Dandapat, and S. Poria. Circular hydraulic jump in generalized-newtonian fluids. arXiv:0809.2231v3 [physics.flu-dyn], 2008.

[54] N. M. Ribe. Periodic folding of viscous sheets. Physical Review E: Statistical, Nonlinear, and Sfoft Matter Physics, 68, 2003.

[55] N. M. Ribe, E. Stutzmann, Y. Ren, and R. van der Hilst. Bucking instabilities of subducted lithosphere beneath the transition zone. International Journal for Numerical Methods in Fluids, 254:173-179, 2007.

[56] W. J. Rider, J. A. Greenough, and J. R. Kamm. Acurate monotonicity and extrema perserving methods through adaptative nonlinear hybridizations. Journal of Computational Physics, 225:1827 - 1848, 2007.

[57] A. Ritter. Die fortplanzung der wassserwellen. Zeitschrift Verein Deutscher Ingenieure, 36:947-954, 1982.

[58] P. L. Roe. Some contribution to the modelling of discontinuous flows. Lectures in Applied Mathematics. Large-scale Computations in Fluid Mechanics, 22:163, 1985.

[59] P. L. Roe. Characteristic-based schemes for the euler equations. Annual Reviews Fluid Mechanic, 18:337-365, 1986.

[60] P. L. Roe and J. Pike. Efficient construction and utilisation of approximate riemann solutions. Computing in Applied Sciences and Engineeting, 6:499-518, 1984.

[61] P.L. Roe and M. J. Baines. Algorithms for advection and shock problems. Proceedings of the Fourth GAMM Conference on Numerical Methods in Fluid Mechanics, 5:281, 1982.

[62] J. Shi, Y. T. Zhang, and C. W. Shu. Resolution of high-order WENO schemes for complicated flow structures. Journal of Computational Physics, 186:690-696, 2003.

[63] C. W. Shu and S. Osher. Efficient implementation of essentially non-oscilatory shock capturing schemes. Journal of Computational Physics, 83:32, 1989. 
[64] C. W. Shulz-Rinne, J. P. Collins, and H. M. Glaz. Numerical solution of the riemann problem for two-dimensional gas dynamics. SIAM Journal of Scientific Computing, 14:1394-1414, 1993.

[65] D. B. Spalding. A novel finite difference formulation for differential expressions involving both first and second derivatives. International Journal for Numerical Methods in Fluids, 4:551-559, 1972.

[66] S. P. Spekreijse. Multigrid solution of monotone second-order discretizations of hyperbolic conservation laws. Mathematics of Computation, 49:135-155, 1987.

[67] P. K. Sweby. High resolution schemes using flux limiters for hyperbolic conservation laws. SIAM Journal on Numerical Analysis, 21:995-1011, 1884.

[68] G. I. Taylor. Low raynolds number flows. National Committee for Fluid Mechanics Films-Illustrated experiments in fluid mechanics, 1974.

[69] Z. Teng. Exact boundary conditions for the initial value problem of convex conservation laws. Journal of Computational Physics, 229:3792â3801, 2010.

[70] D. W. Thoe and E. C. Zachmanoglou. Introduction to Partial Differential Equation with aplications. 1986.

[71] M. F. Tomé, A. C. Filho, J. A. Cuminato, N. Mangiavacchi, and S. McKee. GENSMAC3D: a numerical method for solving unsteady three-dimensional free surface flows. International Journal for Numerical Methods in Fluids, 37:747-796, 2001.

[72] M. F. Tomé and S. McKee. A computational marker-and-cell method for free surface flows in general domains. Journal of Computational Physics, 110:171-186, 1994.

[73] E. F. Toro. Rieman solvers and numerical methods for fluids dynamics. New York, 1999.

[74] L. N. Trefethen and D. Bau. Numerical Linear Algebra. Society for Industrial and Applied Mathematics, 1997.

[75] G. D. van Albada, B. van Leer, and W.W. Roberts. A comparative study of computational methods in cosmic gas dynamics. Astrononomy and Astrophysics, 108:76, 1982.

[76] B. van Leer. Towards the ultimate conservative difference scheme. I. The quest of monotonicity. Lecture Notes in Physics, 18:163-168, 1973.

[77] B. van Leer. Towards the ultimate conservative difference scheme. II. Monotonicity and conservation combined in a second-order scheme. Journal of Computational Physics, 14:361, 1974. 
[78] B. van Leer. Towards the ultimate conservative difference scheme. IV.A new approach to numerical convection. Journal of Computational Physics, 23:276-299, 1977.

[79] B. van Leer. Towards the ultimate conservative difference scheme. V. A second-order sequel to Godunov's method. Journal of Computational Physics, 33:101-136, 1977.

[80] L. Ville, L. Silva, and T. Coupez. Convected level set method for numerical simulation of fluid buckling. International Journal for Numerical Methods in Fluids, 66:324-344, 2010.

[81] N. P. Waterson and H. Deconinck. Design principles for bounded higher-order convection schemes - a unified approach. Journal of Computational Physics, 224:182-207, 2007.

[82] E. J. Watson. The radial spread of a liquid jet over a horizontal plane. Journal of Fluid Mechanics, 20:481-499, 1964.

[83] P. Woodward and P. Collela. The numerical simulation of two-dimensional fluid flow with strong shocks. Journal of Computational Physics, 54:115-173, 1984.

[84] N. K. Yamaleev and M. H. Carpenter. Third-order energy stable weno scheme. Journal of Computational Physics, 228:3025-3027, 2009.

[85] M. Zijlema. On the construction of a third-order accurate monotone convection scheme with application to turbulent flows in general domains. International Journal for Numerical Methods in Fluids, 22:619-641, 1996. 